\title{
Alloreactive natural killer cell therapy for multiple myeloma
}

Citation for published version (APA):

Sarkar, S. (2014). Alloreactive natural killer cell therapy for multiple myeloma. [Doctoral Thesis, Maastricht University]. Maastricht University. https://doi.org/10.26481/dis.20141111ss

Document status and date:

Published: 01/01/2014

DOI:

10.26481/dis.20141111ss

Document Version:

Publisher's PDF, also known as Version of record

\section{Please check the document version of this publication:}

- A submitted manuscript is the version of the article upon submission and before peer-review. There can be important differences between the submitted version and the official published version of record.

People interested in the research are advised to contact the author for the final version of the publication, or visit the DOI to the publisher's website.

- The final author version and the galley proof are versions of the publication after peer review.

- The final published version features the final layout of the paper including the volume, issue and page numbers.

Link to publication

\footnotetext{
General rights rights.

- You may freely distribute the URL identifying the publication in the public portal. please follow below link for the End User Agreement:

www.umlib.nl/taverne-license

Take down policy

If you believe that this document breaches copyright please contact us at:

repository@maastrichtuniversity.nl

providing details and we will investigate your claim.
}

Copyright and moral rights for the publications made accessible in the public portal are retained by the authors and/or other copyright owners and it is a condition of accessing publications that users recognise and abide by the legal requirements associated with these

- Users may download and print one copy of any publication from the public portal for the purpose of private study or research.

- You may not further distribute the material or use it for any profit-making activity or commercial gain

If the publication is distributed under the terms of Article $25 \mathrm{fa}$ of the Dutch Copyright Act, indicated by the "Taverne" license above, 
Alloreactive Natural Killer cell therapy for Multiple Myeloma 
Dedicated to every Myeloma patient considering NK cell therapy. Dedicated to a visionary and my role model PM Narendra Modi.

Copyright: Subhashis Sarkar, Maastricht 2014

No part of this book may be reproduced or transmitted in any form or by any means, without prior permission in writing by the author, or when appropriate, by the publishers of the publications.

Layout: Tiny Wouters

Cover: Subhashis Sarkar

Production: CPI Koninklijke Wöhrmann B.V., Zutphen

ISBN: 978-94-6203-688-8 


\title{
Alloreactive Natural Killer cell therapy for Multiple Myeloma
}

\author{
PROEFSCHRIFT
}

ter verkrijging van de graad van doctor aan de Universiteit Maastricht, op gezag van de Rector Magnificus, Prof. dr. L.L.G. Soete volgens het besluit van het College van Decanen, in het openbaar te verdedigen op dinsdag 11 november 2014 om 10.00 uur door

\section{Subhashis Sarkar}

Born on 1 May 1986 in Kolkata, India 


\section{Promotor}

Prof. dr. G.M.J. Bos

\section{Co-promotores}

Dr. W.T.V. Germeraad

Dr. L. Wieten

\section{Beoordelingscommissie}

Prof. dr. P. Lambin (voorzitter)

Prof. dr. E.A.L. Biessen

Prof. dr. P.J. Peters

Prof. dr. K. Satyamoorthy, Professor \& Head, Department of Biotechnology, Manipal Life Sciences Centre, Manipal, INDIA

Prof. dr. S. Zweegman, Department of Hematology, VU University Medical Center, Amsterdam 


\section{CONTENTS}

$\begin{array}{lll}\text { Chapter } 1 & \text { General introduction } & 7\end{array}$

Chapter 2 Hypoxia induced impairment of NK cell cytotoxicity against

Multiple Myeloma can be overcome by IL-2 activation of the NK cells

Chapter 3 Optimal selection of Natural Killer cells to kill myeloma: the role of HLA-E and NKG2A

Chapter 4 Alloreactive Natural Killer cells have anti-tumor capacity against disseminated human Multiple Myeloma in RAG2-/-pc-/- mice when combined with low dose cyclophosphamide and total body irradiation

Chapter 5 The synergistic effect of immunomodulatory drugs or monoclonal 101 antibodies on Natural Killer cell mediated anti-Multiple Myeloma responses is lost in an hypoxic environment

Chapter 6 Discussion

Summary

Acknowledgement

Curriculum Vitae

Valorization 



\section{Chapter 1}

General introduction 
Chapter 1 


\section{Introduction}

Hematopoietic stem cells in the bone marrow give rise to several lineages of blood cells, including leukocytes - cells that make up our immune system to kill invaders from outside-, red blood cells - for oxygen transport - and thrombocytes - necessary for accurate blood clotting. In a multistep developmental process, depending on environmental factors, cells differentiate to the next stage thereby loosing potential to still become other types of cells. Along one of those lineages, B lymphocytes will develop. When these $B$ cells encounter an antigen i.e from a virus or bacteria, a process taking place in lymphoid organs, they become antibody secreting plasma cells, that ultimately will home back to the bone marrow ${ }^{1}$. Antibodies or immunoglobulin's are key mediators of humoral adaptive immune responses during viral and bacterial infections, but they also are important in anti-tumor responses ${ }^{2}$. These immunoglobulin's are composed of light chains and heavy chains. Several heavy chains do exist that determine the isotype, as well as two light chains. Every plasma B cell does produce one type of immunoglobulin (i.e. IgG or IgA with one type of light chain only). The combination of heavy chain and light chain together determine the idiotype, a structure that determines the antigen specificity of antibodies (Figure 1.1).

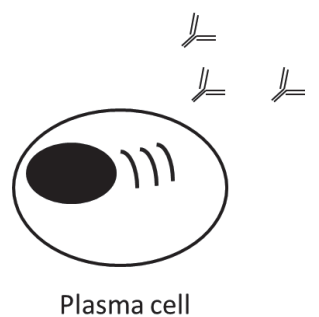

Plasma cell

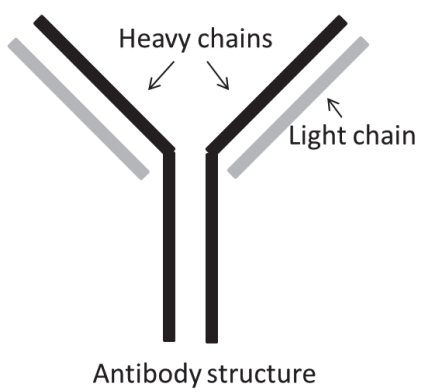

Antibody structure

Figure 1.1 Plasma cell and antibody structure.

Plasma cells secrete antibodies. The antibodies are composed of heavy and light chains.

During the continuous process of differentiation and maturation, genetic changes can occur leading to malignant transformation of plasma cells. If a patient carries a large number of malignant plasma cells that finally leads to symptoms, it results in a disease that is called Multiple Myeloma or Kahler's disease. In about half of the cases of Multiple Myeloma an oncogene is linked to the immunoglobulin heavy chain gene (located on chromosome 14), that induces uncontrolled cell proliferation. In a substantial number of patients, a trisomy of chromosomes can be detected, also contributing to abnormal cell proliferation. In later stages of malignant transformation, 
additional genetic and epigenetic events do occur that finally lead to an increase of malignant plasma cells and symptomatic disease ${ }^{3-7}$.

Apart from intrinsic abnormalities of the plasma cells, bone marrow derived factors, which attract plasma cells to the bone marrow also play a role in survival of malignantly transformed cells. These microenvironments in the bone marrow harbor a complex interplay of plasma cells, lymphocytes, fibroblasts, osteoclasts, osteoblasts and the vascular structure in the bone marrow. The interaction of all these cells is regulated both by direct cell-cell contact via adhesion molecules and cytokines produced by these cells (Figure 1.2). One of the most characteristic features of Multiple Myeloma that is caused by these pathophysiological mechanisms is the occurrence of osteolysis due to a disbalance in osteoblast (that form bone) and osteoclast (that resolve bone) activity ${ }^{3}$.

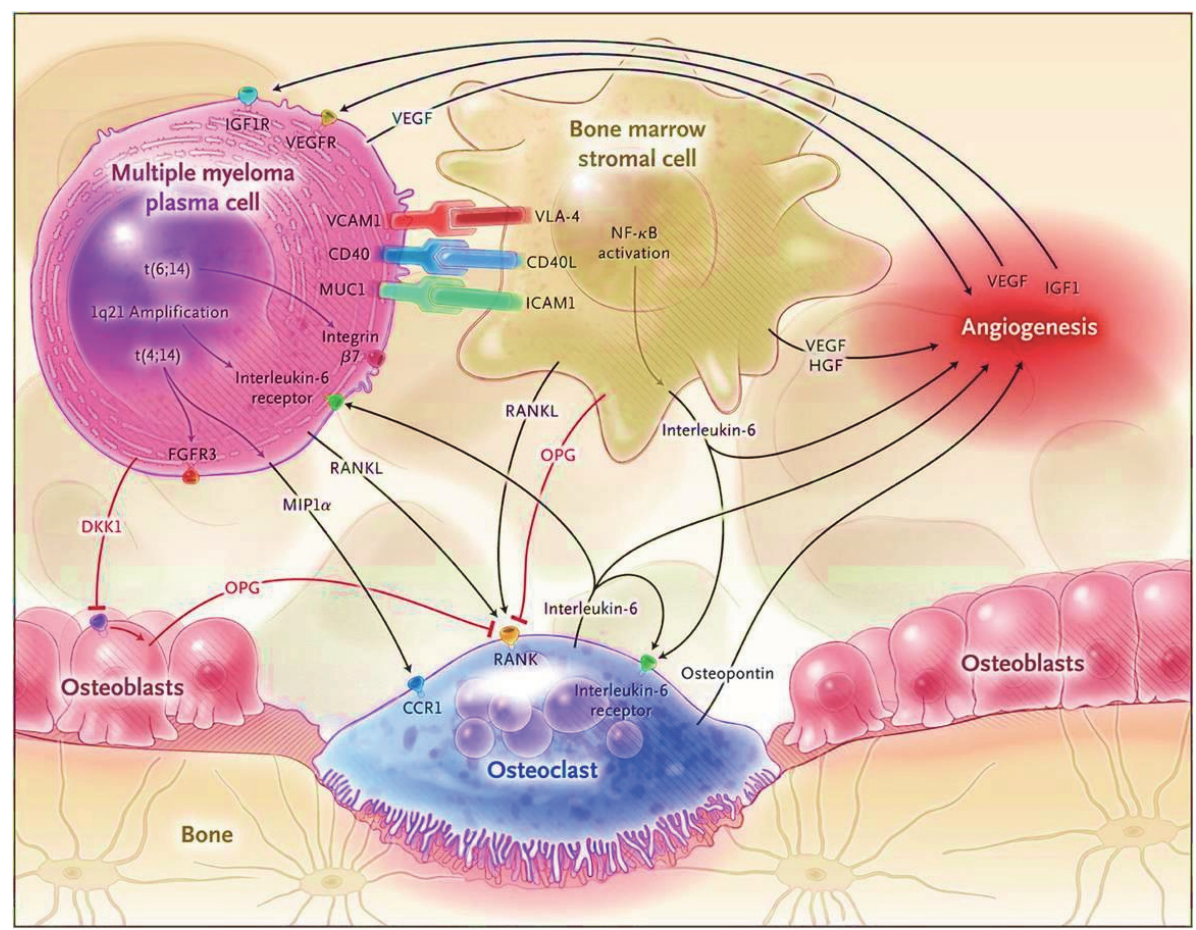

Figure 1.2 Myeloma bone marrow microenvironment.

Myeloma cell reside with bone marrow. Within bone marrow other accessory cells such as osteoclast, osteoblast and stromal cell assist in myeloma outgrowth. These cells together with myeloma cell secrete diverse soluble factors which promote myeloma cell growth. Adapted with permission from K. Anderson (NEJM 2011;364:1046-1060). 


\section{Multiple Myeloma}

Multiple Myeloma accounts for about $13 \%$ of hematological cancers and is responsible for $0.9-1.2 \%$ of deaths from cancer $^{8}$. Most people are above the age of 65 when the diagnosis is made. The incidence is higher in man than in women and more frequent in people with an Afro-Caribbean background than for Caucasians. In India, the median age of patients at diagnosis stands at 55 years, 10 years lower than the western world ${ }^{9}$. The reported incidence of Multiple Myeloma in India ranges from 0.5-1.2 per 100,000, while the median age of survival is only 30 months $^{10}$. Thus far there are no hereditary or environmental factors identified that might cause Multiple Myeloma, though suggestions have been made that meat intake, fat intake and body weight might be relevant ${ }^{11}$.

The diagnosis of Multiple Myeloma is characterized by a set of criteria: the presence of monoclonal plasma cells in the bone marrow (monoclonality can usually be demonstrated by the observation that the plasma cells only express kappa or lamda light chains, where a normal ratio of these light chains is usually $60 \% / 40 \%$ ) and the presence of monoclonal antibodies (mostly $\lg$ or $\lg A$ ) and/or free light chain protein in the blood are the hallmarks of the diagnosis.

A substantial number of persons (about $5 \%$ of the elderly) do have a monoclonal antibody in their blood reflecting the presence of monoclonal plasma cells. Only those people that present with specific symptoms (anemia - due to cytokine activity that induces imperfect production of red blood cells - , osteolysis as demonstrated by radiographic procedures, - possibly with clinical bone-pain - , hypercalcemia - both due to the disbalance of osteoclasts and osteoblasts - and/or kidney dysfunction usually due to overload of light chains in the kidney tubuli - are considered to suffer from symptomatic Multiple Myeloma. Patients without organ damage are classified as MGUS - monoclonal gammopathy of undetermined significance or asymptomatic myeloma, depending on the number of malignant plasma cells and titer of antibodies present ${ }^{12}$.

The clinical diagnosis is made by performing a bone marrow aspiration to analyze the presence of malignant plasma cells with a specific phenotype ${ }^{13}$, blood analysis monoclonal antibodies and/or monoclonal light chains, calcium levels, hemoglobin level and kidney function - and analysis of bone destruction (osteolysis) of all the marrow containing bones. This latter is usually performed by conventional X-rays, though newer technologies are more and more used ${ }^{14}$.

\section{Current standard therapy for Multiple Myeloma}

After patients are diagnosed with Multiple Myeloma, they are treated with a combination of frontline chemotherapy, which includes a variety of drugs. Thus far, 
there is no option to cure Multiple Myeloma. Therefore, the effort is directed to improve overall survival and maintaining quality of life. Over the years, alkylating drugs - that interfere with DNA synthesis and therefore ability for cells to divide, i.e melphalan and cyclophosphamide - and steroids are the corner stones of anti-myeloma treatment. However, in the last decade, several drugs have become available that also have an anti-myeloma effect and improve survival of the patients. Among them, bortezomib, thalidomide and lenalidomide are the most important ones. Bortezomib is a proteasome inhibitor ${ }^{15}$, which disrupts several pathways of the myeloma cells including NF-k $\beta$ signaling, interferes with neo-angiogenesis, and autocrine and paracrine loops of cytokine signaling. This way, the process of myeloma development is blocked. A second-generation proteasome inhibitor carfilzomib has been described as a welcome addition to the therapeutic arsenal, after FDA approval in $2012^{16}$.

Thalidomide and its more recent analogue lenalidomide are anti-angiogenic molecules, thereby disrupting the myeloma environment and tumor growth. These compounds were later found to have additional functions in boosting several immune cells, especially $\mathrm{T}$ cells and Natural Killer (NK) cells with possibly anti-myeloma killing capacity $^{17,18}$.

The precise combination of drugs to be used is depending on age and comorbidity. Young and fit patients are usually treated with a regimen that aims for reduction in myeloma burden and after this induction treatment high dose chemotherapy (melphalan) will follow. An important step prior to this chemotherapeutic procedure is to isolate the patients hematopoietic stem cells (HSCs) and freeze them, since the intensive melphalan treatment will not only destroy tumor cells but also healthy bone marrow stem cells that are needed to produce all blood cells (red blood cells, leukocytes and thrombocytes). Therefore, the high dose melphalan will be followed by add back of the frozen HSCs, a procedure called autologous stem cell transplantation (auto-SCT). Several clinical trials have indicated that this procedure improves overall survival. Despite this intensive treatment schedule, patients will not be cured, though some patients show a long period (years) of progression free survival with good quality of life. The elderly patient or patients with co-morbidity will not be able to undergo this regimen. They will be treated by a combination of less toxic drugs.

Since patients cannot be cured with the drugs currently available, they will always have progression after a certain time of clinical response. At that time point, new drugs of the above-mentioned category can be used or - after very good initial responses - the same drugs can be applied again. The optimal combinations for patients and the order of the use of the several drugs including now the high dose chemotherapy with autoSCT - is a continuous challenge for further research, in the Netherlands usually under the guidance of the Dutch clinical research association for hematological diseases (HOVON). 


\section{Donor blood stem cell transplantation}

A special topic to be mentioned is the procedure of allogeneic (=donor) SCT (allo-SCT) as alternative to auto-SCT. By making use of donor stem cells, one can transplant the donor immune system to the recipient patient. This procedure, that was originally developed to compensate for radiation damage by which peoples' bone marrow stem cells will be destroyed, has the potential to cure malignant diseases by the so called graft (=donor) versus disease response of the immune system. However, this procedure can also induce an unwanted graft versus patient = host effect (GVH) with detrimental effects on healthy organs, mostly skin, gut, liver and lungs. This GVH effect is due to the fact that donors that are selected will still be mismatched for histocompatibility antigens that evoke the donor immune system to respond. Allo-SCT are most effective in some types of leukemia and some forms of (non-) Hodgkin lymphomas especially in patients of younger age, where the balance of GVH disease (GVHD) and graft versus tumor effect is most optimal. Also in patients without cancer, but with congenital diseases of blood forming cells, allo-SCT is an option to replace the diseased stem cells by healthy cells.

Allo-SCT also has the potential to cure MM, but is often accompanied by a lethal graftversus-host response ${ }^{19}$. After a SCT, T and possibly NK cells might facilitate the graftversus-Myeloma (GVM) response, while the same immune T cells (although of different specificity) will also cause the GVHD ${ }^{20}$. Thus far, it has not been possible to separate the wanted GVM effect from the unwanted GVH response. Altogether, the mortality is high and depending on the observation time after transplantation, it is still over $25 \%$. Moreover, for those patients surviving the procedure, there is still progression of disease in the majority of cases. Therefore, only a small minority of patients does have a long period of progression free survival or are cured. At this time point allo-SCT is not considered a standard treatment outside clinical trials in the Netherlands.

\section{Haploidentical stem cell transplantation and Natural Killer cells}

Since the limitations of clinical effect of allo-SCT - not only in Myeloma but also in several other malignant diseases - but also because of the lack of availability of accurate donors to perform an allo-SCT as described above on time, investigators have looked for alternative donors. The use of haploidentical donors, sharing one of the two haplotypes with the patient can considerably overcome this problem of donor availability. Every patient will usually have a donor since parents, siblings, children and some nieces/nephews will share one haplotype (a set of chromosomes with the relevant transplantation antigens, coming from the father or mother) with the patient. Ruggeri et al. also showed in a landmark paper the phenomenon of enhanced graft vs. acute myeloid leukemia ( $A M L)$ response during haploidentical stem cell transplant- 
tation. Surprisingly, the clinical anti-tumor effect was probably due to graft versus tumor effect of the NK cells and not $\mathrm{T}$ cells ${ }^{21}$. Furthermore, haploidentical NK cell transplantation in contrast to haploidentical T-cell transplantation does not induce any side effects including GVHD.

Our group has shown that haploidentical stem cell transplantation together with nonmyeloablative conditioning regimen can cure breast cancer in a 4T1 breast cancer mice model ${ }^{22}$. Subsequent experiments by our group identified donor NK cells as crucial for the graft versus tumor effector cell during haploidentical transplantation in this model $^{23}$. NK cell depletion of the haploidentical graft fully abrogated the anti-tumor effect and only NK cells in the absence of donor stem cells demonstrated similar results, confirming the data of Ruggeri et al. that NK cells from haploidentical donors might have the potency for strong anti-tumor effects, not only in hematological tumors but also in solid tumors.

Haploidentical transplantation has not been introduced as standard of care in clinical practice, at least not in adults. As reviewed by Bayraktar et al., maintaining T cells in the graft has been linked with very high rates of GVHD, whereas T cell-depleted haploidentical transplantation has been restricted by a higher frequency of graft rejection and non-relapse mortality related to opportunistic infections due to delayed immune reconstitution after transplantation ${ }^{24}$. Therefore, most clinicians wait for more optimal protocols to be developed that are able to reconstitute the immunecompetency of the patients after haploidentical transplantation. These protocols could profit from the anti-tumor effect of NK cells.

Nevertheless the observation by Ruggeri et al. and the work of our own group in breast cancer mice models that NK cells can contribute to anti-tumor effects is the background of the research described in this thesis that hopes to answer the question: can donor NK cells induce anti-myeloma effects?

\section{Natural Killer cells}

NK cells are large granular lymphocytes, which can selectively kill virally infected cells and tumor cells without affecting healthy normal cells. The importance of NK cells for anti-viral immunity is illustrated by studies showing that people with NK cell deficiencies have been observed to be highly susceptible to viral infections ${ }^{25,26}$. Human NK cells are characterized by cell surface expression of CD56 in the absence of CD3. NK cells can be broadly classified into two groups: 1 . CD56 ${ }^{\text {high }}$ CD16 ${ }^{\text {low }}$ NK cells, which constitute approximately $10 \%$ of peripheral blood circulating and $90 \%$ of secondary lymphoid organ residing NK cells. The major function of this subset of NK cells is to produce cytokines, e.g. IFN-y, which activates the adaptive immune response during viral infection or tumor outgrowth. 2. $\mathrm{CD}_{5} 6^{\mathrm{dim}} \mathrm{CD} 16^{\text {high }} \mathrm{NK}$ cells, which constitute around $90 \%$ of peripheral blood circulating and $10 \%$ of secondary lymphoid organ 
residing NK cells. CD56 ${ }^{\text {dim }}$ CD16 ${ }^{\text {high }}$ NK cell subset can mount direct cytotoxic action against virally infected or tumor cells ${ }^{27}$.

\section{NK cell recognition of self versus non-self}

NK cells maintain the equilibrium between tolerance to healthy cells and elimination of virally infected or tumor cells by a panel of activating and inhibitory receptors expressed on their cell surface ${ }^{28,29}$. The balance between signaling by the various inhibitory and activating receptors determines whether or not NK cells get activated upon interaction with a potential target cell. Thus, by monitoring for the presence of inhibitory and activating ligands, NK cells can distinguish self versus non-/altered-self (Figure 1.3) and roughly three types of recognition can be distinguished: 1) "NK cell tolerance" for healthy cells; ensured by the relatively high expression of inhibitory NK cell ligands like MHC-class I and low expression of activating ligands on a healthy cell. 2) "Missing-self recognition"; viruses, upon infection of a cell, frequently downregulate MHC class I expression on the cell surface to evade the T cell response. This low expression of $\mathrm{MHC}$ class I on a virally infected cell will reduce the activation threshold of NK cells and allows them to become activated and kill the virally infected cells if sufficient activating signal is provided. 3) "Induced-self recognition"; NK cells respond to infected cells that maintain MHC class I because infected- or stressed cells frequently enhance the expression of activating NK cell ligands to a level high enough to overcome inhibitory signaling by MHC molecules. Like virally infected cells, tumor cells can express reduced levels of MHC class I and/or enhanced levels of activating ligands making them susceptible for killing by NK cells (Figure 1.3).

After recognizing a virally infected or malignantly transformed cell and receiving the proper signals, NK cells initiate the process of cell lysis by secreting granules containing proteins like perforin and granzyme B. Perforin molecules create pores in the cell membrane of tumor cells ${ }^{30}$, thereby facilitating the entry and subsequent downstream functionality of granzyme B. Granzyme B activates the caspase-3 mediated proapoptotic signaling pathways within the tumor cells, ultimately leading to cell death and tumor regression ${ }^{31}$. During this whole process of vesicular release of perforin and granzyme B, intravesicular membrane LAMP-1 (CD107a) also becomes exposed on the cell surface of NK cells ${ }^{32}$, and thus can be used as a surrogate marker for NK cell mediated cytolysis of virally infected or tumor cells during in vitro analysis of tumor cell kill by NK cells ${ }^{33}$. NK cells can also kill a target cell through interaction of death ligands and receptors like FASL-FAS and TRAIL-TRAIL receptor ${ }^{34,35}$. 


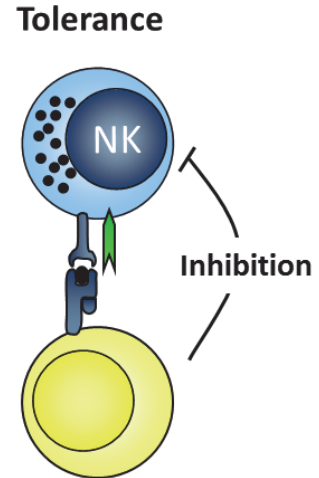

healthy cell
Missing-self

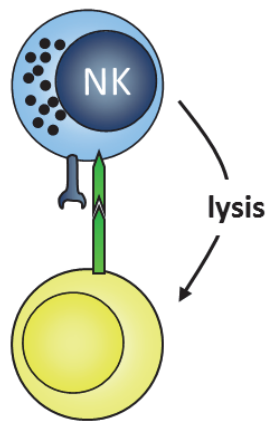

diseased cell
Induced-self

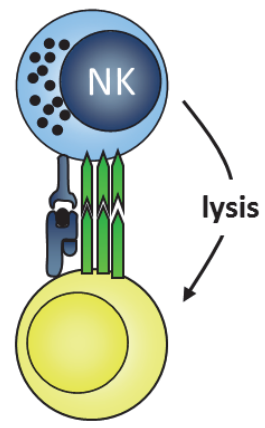

diseased cell

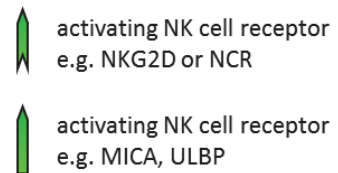

Figure 1.3 NK cell recognition of healthy and tumor cells.

NK cells are not triggered by healthy cells due to lack of "stress induced ligands" such as MIC's and ULBP's. Thus when a NK cell interacts with a healthy cell, the inhibitory signals are greater than activating signals, and consequently NK cells do not kill a healthy cell. This phenomenon is described as NK cell "Tolerance". On the other hand, tumor cells express lower levels of MHC class I and the NK cell lyse the tumor by the mechanism of "Missing self". During this early tumor phase they can also express higher levels of MIC's and and thus can be eliminated by autologous NK cell. This is referred to as the Induced self.

\section{NK cell receptors}

NK cells express a broad array of activating receptors ${ }^{29,36,37}$. Activating receptors on NK cells screen for the presence of "stress induced-flags" specifically present on tumor cells and subsequently trigger NK cell mediated killing. The ligands for these receptors are usually not or barely expressed by healthy cells but, their expression increases in response cellular stress or viral infection. NKG2D, DNAM-1 and the natural cytotoxicity receptors (NCRs) are prototype activating receptors shown to be important for NK cell killing of cells of various types of tumors including myeloma ${ }^{38-40}$. The ligands of NKG2D are self-molecules (i.e. MICA/B, ULBP1-3) and their expression is enhanced upon cellular stress e.g. induction of the DNA-damage response, a pathway associated with tumorigenesis ${ }^{41}$. DNAM-1 interacts with poliovirus receptor (PVR) and nectin- $1^{42}$. The NCR family consists of NKp30, NKp44 and NKp46 and recognizes virally-associated 
proteins like BAT and $\mathrm{BAF}^{43}$. Although the importance of NCRs for anti-tumor NK cell response is evident, the tumor-associated ligands for these receptors remain largely elusive, except for the recently identified NKp30 ligand $\mathrm{B} 7-\mathrm{H}^{44}$. Another potent activating receptor is CD16 that interacts with the Fc parts of an antibody thereby triggering NK cells to lyse a tumor cell coated with monoclonal antibodies by a process called antibody dependent cell cytotoxicity (ADCC) ${ }^{45}$.

Most inhibitory receptors bind to self-molecules like classical and non-classical MHC class I (HLA class I and HLA-E in humans). These molecules are expressed by virtually every healthy cell and protect against unwanted NK cell lysis ${ }^{46}$. The most well known class of inhibitory receptors is the killer immunoglobulin-like receptor (KIR) family interacting with the highly polymorphic HLA class I molecules on normal cells. The KIR family consists of fourteen inhibitory or activating family members each having either two (KIR2D) or three (KIR3D) extracellular immunoglobulin domains. Inhibitory KIRs (KIRxDL) are characterized by a long, inhibitory intracellular signaling domain while their activating counterparts (KIRxDS) express a short activating domain ${ }^{47}$. The HLA class I ligands of the inhibitory KIRs are highly polymorphic and based on the allotypic presence of specific epitopes, inhibitory KIRs each recognize one group of HLA class I alleles (Figure 4.4). The best characterized are: KIR2DL1 ligating group 2 HLA-C alleles characterized by Lysine at position 80, KIR2DL2/3 ligating group 1 HLA-C alleles characterized a asparagine at position 80 and KIR3DL1, recognizing HLA-B and A alleles with Bw4 motifs ${ }^{48,49}$. NKG2A is a separate inhibitory receptor on NK cells interacting with HLA-E on the target cells ${ }^{50-52}$. In contrast to HLA class I, HLA-E is a low-polymorphic non-classical major histocompatibility class I molecule and the two existing functional alleles both interact with the CD94-NKG2A complex. HLA-E presents peptides derived from classical $\mathrm{MHC}$ class I molecules on its peptide binding groove ${ }^{53}$.
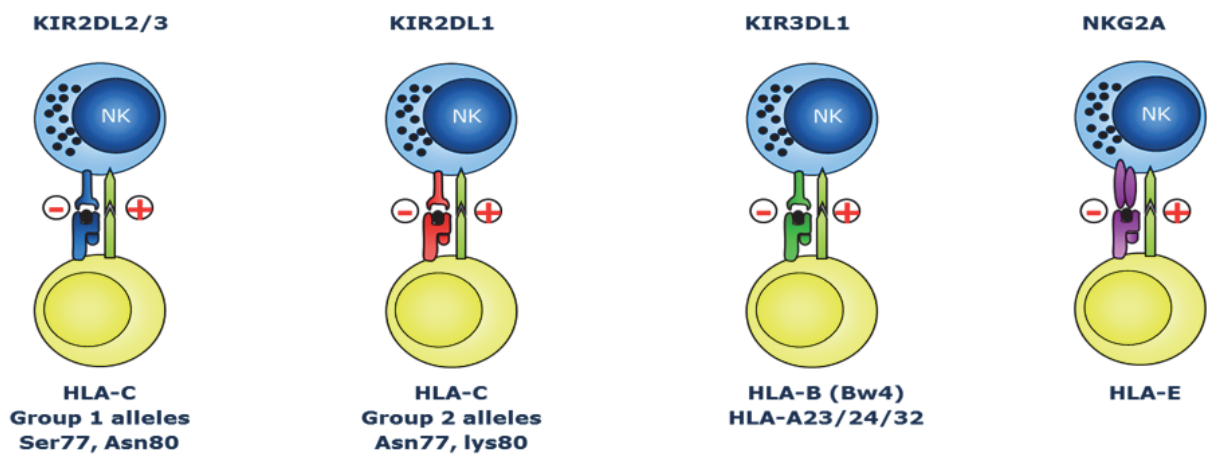

Figure 1.4 NK cell inhibitory receptors.

NK cells express two kinds of inhibitory receptors, Killer Inhibitory Receptors (KIRs) and NKG2A. KIRs on NK cell bind to MHC class I molecules on the target cell, while NKG2A interacts with HLA-E. Peripheral blood derived NK cells can have one or more of these inhibitory receptors in any possible combination, thus generating 16 diverse NK cell populations based on interaction with HLA. 


\section{NK cell alloreactivity}

KIRs, located on chromosome 19, and HLA, located on chromosome 6, are inherited independently ${ }^{54}$. Consequently, an individual can express a particular KIR while the HLA ligand is missing. Due to the existence of a process called NK cell "licensing", autoreactivity by those ligand missing NK cells does not occur. NK cell licensing, implies that only NK cells that have previously seen their HLA-ligand, e.g. during maturation, are licensed to kill while NK cells expressing KIRs for which the ligand is missing remain hyporesponsive ${ }^{55,56}$. Ruggeri et al. showed that the anti-tumor effect of NK cells in the haploidentical SCT setting in AML could be attributed to the presence of "alloreactive NK cells" ${ }^{21}$. Alloreactive NK cells express KIRs for which the HLA ligand is missing in the patient ${ }^{21}$. These KIR-ligand mismatched NK cells can kill tumor cells based on "missing self recognition" if they have been licensed for the ligand mismatched KIR. Thus, an ideal alloreactive NK cell donor should express one or more HLA epitopes that are absent in the patient, and expresses the specific KIR that can interact with the missing HLA-epitope (e.g. a donor is $\mathrm{C} 1+, \mathrm{C} 2+$ and $\mathrm{Bw} 4+$ while the patient is $\mathrm{C} 1+, \mathrm{C} 2$ - and $\mathrm{Bw} 4+$, meaning that KIR2DL1 is the mismatched and licensed KIR).

Inhibitory NK cell receptors like KIRs and NKG2A are germline encoded and NK cells can clonally express one or a combination of inhibitory receptors ${ }^{57}$ leading to various NK cell subsets that can each be inhibited by different HLA ligands. Therefore, only a fraction of the NK cells will exclusively express ligand mismatched KIRs, and has the full ability to kill based on missing self. Preferably, these NK cells do not express NKG2A because NKG2A interacts with all known functional HLA-E alleles and HLA-E is normally expressed on every HLA class I positive cell. Also in a clinical setting where only the NK cells will be infused, allogeneic NK cells from a healthy donor can be obtained that express KIRs that do not recognize the HLA class I molecules on patient tumor cells (KIRligand mismatch) thereby minimizing the inhibition, and significantly escalating NK cell cytolytic functions.

\section{Adoptive NK cell therapy for Multiple Myeloma}

To exploit the anti-tumor potential of NK cells, multiple studies aimed at enhancing the anti-myeloma capacity of autologous NK cells in mouse models and in myeloma patients. In the early years, this was done was done by in vivo administration of NK cell activating cytokines (e.g. interferons and IL-2). More recently, it became clear that immunomodulatory drugs (e.g. lenalidomide) and proteasome inhibitors can also augment NK cell activity. These studies were, however, not highly successful or were accompanied by significant toxicities. These studies have been summarized by Godfrey et al., and their detailed discussion is beyond the scope of this introduction ${ }^{58}$. 
An alternative approach is to adoptively transfer NK cells and this approach has been studied in various pre-clinical murine models. One of the models is the 5T33MM model in C57BL/KaLwRij mice, which is a well-established murine myeloma model ${ }^{59,60}$, where the influence of hypoxia has been described ${ }^{61}$. Alici et al. demonstrated that infused autologous mouse NK cells could effectively mount anti-myeloma response in the 5T33 murine myeloma mode ${ }^{62}$. This anti-myeloma response was depending on the number of infused NK cells and could be improved by the co-administration of IL-2. A major limitation of this being a murine system is that it does not allow study of the antimyeloma response of human NK cells.

Immunodeficient mice, e.g. NOD-scid IL2R ${ }^{\text {null }}$ (NSG) mice, lack mature lymphocytes (i.e. T cells, B cells and NK cells). Therefore, such mice models offer the advantage of determining the response of human NK cells against human myeloma cells. In a first study, Swift et al. have demonstrated that a NK cell line, i.e. NK-92, can reduce myeloma burden in NSG mice ${ }^{63}$. However, such clonal cell lines for therapeutic applications always bear the risk of clonal proliferation and clonal cell malignancy. A second study has shown that NK cells expanded in co-culture with K562 cells modified to express membrane bound IL-15 and 41BB ligand inhibited growth of a myeloma cell line (OPM2) in culture and of primary myeloma cells in NSG mice ${ }^{64}$. The major disadvantage of this system is the unreasonably high number of allogeneic NK cells $\left(160 \times 10^{6}\right)$ needed to cure mice with myeloma in a human fetal bone within the skin. Lower number of NK cells $\left(40 \times 10^{6}\right)$ did not show any therapeutic activity. Translating such a system from mice to human will be extremely challenging and requires impossibly high numbers of NK cells. For clinical purposes, focus is further on generation of large quantities of NK cells either from CD34+ hematopoietic progenitor cells $^{65,66}$, blood derived NK cells ${ }^{67}$, pluripotent stem cells ${ }^{68}$, and placental derived NK cells $^{69}$. However, much remains to be established about the efficacy of these products in targeting myeloma in an extremely immunoevasive bone marrow microenvironment. So far, only one study investigated the effect of NK cell infusion in human myeloma patients. In this pioneering study, Shi et al. infused haploidentical, KIR-ligand mismatched, IL-2 activated NK cells into myeloma patients in combination with high dose melphalan and fludaribine treatment and a delayed rescue with autologous stem cells $^{70}$. Although the clinical efficacy needs to be improved this study encouragingly demonstrated the feasibility and safety of the approach.

\section{Tumor and microenvironment derived factors suppressing NK cell functionality}

During (hematological) tumor progression, the tumor cells and their microenvironment acquire multiple strategies to evade NK cells ${ }^{71}$. One of these strategies is the production of immunosuppressive soluble factors by myeloma cells, infiltrating immune cells or 
bone marrow associated cells. For example TGF- $\beta$, increasingly released and activated during Multiple Myeloma disease progression, promotes bone destruction ${ }^{72}$. At an immunological level, TGF- $\beta$ can provide significant assistance to tumor cells in impairing NK cell cytotoxicity and cytokine production ${ }^{73}$. Blocking of TGF- $\beta$ can be a therapeutic approach to restore NK cell cytotoxicity ${ }^{74}$. Another example is IL-6, the major cytokine in myeloma that promotes disease progression. IL- 6 has also been shown to decrease NK cell functionality ${ }^{75}$. M-proteins secreted by myeloma cells also diminish NK cell effector functions ${ }^{76}$; MICA on myeloma cells interacts with NKG2D, and subsequently triggers NK cytotoxicity. However, MICA is shed and secreted into the serum of myeloma patients, again highlighting the immunoevasive strategies employed by myeloma cells ${ }^{77}$. Another potential inhibitory factor is prostaglandin E2 (PGE2), which can be produced by bone marrow stromal cells or myeloma ${ }^{78}$ and has recently been shown by us to potently inhibit NK cell cytotoxicity ${ }^{79}$. In addition to soluble factors, NK cells from myeloma patients have been observed to have low expression of key activating receptors such as NKG2D, NKp30 and DNAM-1 and CD16 ${ }^{80}$, the latter can also cause poor responses to monoclonal antibody therapy. On the other hand, myeloma cells have been shown to express increased levels of inhibitory cell surface molecules such as PD-L1 ${ }^{81}$ or decreased levels of activating ligands for DNAM ${ }^{82}$ and NKG2D ${ }^{83}$. Additionally, a critical factor, which is usually overlooked while developing NK cell based immunotherapeutic approaches, is the low oxygen concentration or hypoxia in the tumor microenvironment.

\section{Multiple Myeloma, tumor hypoxia and the bone marrow microenvironment}

Hypoxia is a condition when there is not sufficient oxygen supply to the rapidly multiplying tumor cells. Hypoxia is a phenomenon commonly observed in solid tumors ${ }^{84}$. During rapid multiplication of tumor cells, there is formation of a new blood supply, neo-angiogenesis, to supply nutrients and oxygen. However, tumor cell proliferation can be so rapid that neo-angiogenesis cannot catch up. This leads to the formation of an inner core of tumor cells, which have no blood supply, and consequently no adequate oxygen supply. When the oxygen concentration in such regions of a tumor is lower than $1.3 \%$ oxygen, it is considered hypoxic ${ }^{85,86}$. These hypoxic areas within solid tumors diminish the apoptotic potential of tumor cells thereby promoting their survival ${ }^{87}$.

The myeloma microenviroment is also characterized by hypoxia. Myeloma cells reside within the bone marrow, and this anatomical region is difficult to access directly to measure oxygen concentration. However, it has been shown that bone marrow has extensive regions with extremely hypoxic niches, which is a basic necessity for maintenance of hematopoiesis ${ }^{88}$. In the 5 T33MM model, the bone marrow of myeloma 
bearing mice has been shown to be more hypoxic than the bone marrow of healthy mice ${ }^{89}$. Furthermore, in multiple studies with human myeloma patient derived bone biopsies, expression of hypoxia inducible factor (HIF-1 $\alpha$ ) has been observed ${ }^{90,91}$. HIF-1 $\alpha$ is a protein that is rapidly expressed when oxygen concentrations drop below $1.3 \%$. Also in bone marrow aspirates from myeloma patients, low oxygen levels have been measured. Although these levels did not significantly differ from the levels observed in aspirates of control persons, it illustrates the presence of hypoxic niches in the human bone marrow ${ }^{92}$. Combined with the extreme chemoresistance seen in solid tumors residing in hypoxic environments, it becomes imperative to develop anti-myeloma immunotherapy, which target myeloma cells in their hypoxic microenvironment.

\section{Outline of the thesis}

Despite recent advances, Multiple Myeloma remains an incurable disease. NK cell therapy is a promising option for providing a possible cure for this disease. To achieve this, our goal was to identify inhibitory factors which impair NK cell cytolytic functions, and subsequently investigate approaches to overcome such inhibitory factors. The inhibitory factors we aimed to investigate could be classified into myeloma microenvironmental factors such as bone marrow hypoxia in chapter 2, and at cellular level the resistance provided by myeloma cells to NK cell killing in the form of MHC class I and HLA-E in chapter 3. In chapter 3, we additionally aimed to identify NK cell subsets, which can evade the strong inhibitory signals provided by myeloma cells. In chapter 4, our goal was to investigate the anti-myeloma potential of allogeneic NK cells in a disseminated myeloma model in $\mathrm{RAG2}^{-/-} \mathrm{\gamma c}^{-/-}$mice. In chapter 5 , we aimed to study the NK cell boosting potential of some of the new generation immunomodulatory antimyeloma chemotherapeutics or monoclonal antibodies under hypoxic conditions, to further consolidate our NK cell therapy for Multiple Myeloma. In chapter 6, a general discussion is held on the impact of our findings and outlining a future perspective for adoptive NK cell therapy. 


\section{REFERENCES}

1. Fairfax KA, Kallies A, Nutt SL, Tarlinton DM (2008) Plasma cell development: from B-cell subsets to longterm survival niches. Semin Immunol 20: 49-58.

2. Delves PJ, Roitt IM (2000) The immune system. First of two parts. N Engl J Med 343: 37-49.

3. Palumbo A, Anderson K (2011) Multiple myeloma. N Engl J Med 364: 1046-1060.

4. Koura DT, Langston AA (2013) Inherited predisposition to multiple myeloma. Ther Adv Hematol 4: 291-297.

5. Chubb D, Weinhold N, Broderick P, Chen B, Johnson DC, et al. (2013) Common variation at 3q26.2, $6 p 21.33,17 p 11.2$ and 22q13.1 influences multiple myeloma risk. Nat Genet.

6. Avet-Loiseau H, Attal M, Moreau P, Charbonnel C, Garban F, et al. (2007) Genetic abnormalities and survival in multiple myeloma: the experience of the Intergroupe Francophone du Myelome. Blood 109: 3489-3495.

7. Roccaro AM, Sacco A, Thompson B, Leleu X, Azab AK, et al. (2009) MicroRNAs 15a and 16 regulate tumor proliferation in multiple myeloma. Blood 113: 6669-6680.

8. Parkin DM, Bray F, Ferlay J, Pisani P (2005) Global cancer statistics, 2002. CA Cancer J Clin 55: 74-108.

9. National Cancer Registry Programme (2005) Two year report of the Population based Cancer registries 1999-2000. New Delhi: Indian Council of Medical Research.

10. Nair MK, Varghese C, Krishnan E, Sankaranarayanan R, Nair B (1993) Survival in multiple myeloma in Kerala. Natl Med J India 6:7-10.

11. Food, Nutrition, physical activity and the prevention of cancer: a global perspective, World cancer research fund, 2007.

12. Smith D, Yong K (2013) Multiple Myeloma. BMJ 346: doi: 10.1136/bmj.f3863.

13. Bataille R, Jégo G, Robillard N, Barillé-Nion S, Harousseau JL et al. (2006) The phenotype of normal, reactive and malignant plasma cells. Identification of "many and multiple myelomas" and of new targets for myeloma therapy. Haematologica 91: 1234-1240.

14. Regelink JC, Minnema MC, Terpos E, Kamphuis MH, Raijmakers PG et al. (2013) Comparison of modern and conventional imaging techniques in establishing multiple myeloma-related bone disease: a systematic review. Br J Haematol 162: 50-61.

15. Richardson PG, Barlogie B, Berenson J, Singhal S, Jagannath S, et al. (2003) A phase 2 study of bortezomib in relapsed, refractory myeloma. N Engl J Med 348: 2609-2617.

16. Kortuem KM, Stewart AK (2013) Carfilzomib. Blood 121: 893-897.

17. Mitsiades N, Mitsiades CS, Poulaki V, Chauhan D, Richardson PG, et al. (2002) Apoptotic signaling induced by immunomodulatory thalidomide analogs in human multiple myeloma cells: therapeutic implications. Blood 99: 4525-4530.

18. Chou T (2012) Multiple myeloma : recent progress in diagnosis and treatment. J Clin Exp Hematop 52: 149-159.

19. Lokhorst HM, Schattenberg A, Cornelissen JJ, Thomas LL, Verdonck LF (1997) Donor leukocyte infusions are effective in relapsed multiple myeloma after allogeneic bone marrow transplantation. Blood 90 : 4206-4211.

20. Shlomchik WD (2007) Graft-versus-host disease. Nat Rev Immunol 7: 340-352.

21. Ruggeri L, Capanni M, Urbani E, Perruccio K, Shlomchik WD, et al. (2002) Effectiveness of donor natural killer cell alloreactivity in mismatched hematopoietic transplants. Science 295: 2097-2100.

22. Vanclée A, van Gelder M, Schouten HC, Bos GM (2006) Graft-versus-tumor effects on murine mammary carcinoma in a model of nonmyeloablative haploidentical stem cell transplantation. Bone Marrow Transplant 37: 1043-1049.

23. Frings PW, van Elssen CH, Wieten L, Matos C, Hupperets PS et al. (2011) Elimination of the chemotherapy resistant subpopulation of 4T1 mouse breast cancer by haploidentical NK cells cures the vast majority of mice. Breast Cancer Res Treat. 30: 773-781.

24. Bayraktar UD, Champlin RE, Ciurea SO (2012) Progress in haploidentical stem cell transplantation. Biol Blood Marrow Transplant 18: 372-380.

25. Orange JS. Unraveling human natural killer cell deficiency. J Clin Invest 122:798-801. 
26. Eidenschenk C, Dunne J, Jouanguy E, Fourlinnie C, Gineau L, et al. (2006) A novel primary immunodeficiency with specific natural-killer cell deficiency maps to the centromeric region of chromosome 8. Am J Hum Genet. 78: 721-727.

27. Langers I, Renoux VM, Thiry M, Delvenne P, Jacobs N (2012) Natural killer cells: role in local tumor growth and metastasis Biologics 6: 73-82.

28. Bryceson YT, March ME, Ljunggren HG, Long EO (2006) Activation, coactivation, and costimulation of resting human natural killer cells. Immunol Rev 214: 73-91.

29. Vivier E, Raulet DH, Moretta A, Caligiuri MA, Zitvogel L, et al. (2011) Innate or adaptive immunity? The example of natural killer cells. Science 331: 44-49.

30. Trapani JA, Smyth MJ (2002) Functional significance of the perforin/granzyme cell death pathway. Nat Rev Immunol 2: 735-747.

31. Ida H, Utz PJ, Anderson P, Eguchi K (2005) Granzyme B and natural killer (NK) cell death. Mod Rheumatol 15: 315-322.

32. Krzewski K, Gil-Krzewska A, Nguyen V, Peruzzi G, Coligan JE (2013) LAMP1/CD107a is required for efficient perforin delivery to lytic granules and NK-cell cytotoxicity. Blood 121: 4672-4683.

33. Alter G, Malenfant JM, Altfeld M (2004) CD107a as a functional marker for the identification of natural killer cell activity. J Immunol Methods 294: 15-22.

34. Mori S, Jewett A, Murakami-Mori K, Cavalcanti M, Bonavida B (1997) The participation of the Fas mediated cytotoxic pathway by natural killer cells is tumor-cell-dependent. Cancer Immunol Immunother 44: 282-290.

35. Kim HR, Park HJ, Park JH, Kim SJ, Kim K, Kim J et al. (2004) Characteristics of the killing mechanism of human natural killer cells against hepatocellular carcinoma cell lines HepG2 and Hep3B. Cancer Immunol Immunother 53: 461-470.

36. Moretta A, Bottino C, Vitale M, Pende D, Cantoni C et al. (2001) Activating receptors and coreceptors involved in human natural killer cell-mediated cytolysis. Annu Rev Immunol 19: 197-223.

37. Pegram HJ, Andrews DM, Smyth MJ, Darcy PK, Kershaw MH (2011) Activating and inhibitory receptors of natural killer cells. Immunol Cell Biol 89: 216-224.

38. Carbone E, Neri P, Mesuraca M, Fulciniti MT, Otsuki T et al. (2005) HLA class I, NKG2D, and natural cytotoxicity receptors regulate multiple myeloma cell recognition by natural killer cells. Blood 105: 251-258.

39. Bernal M, Garrido P, Jiménez P, Carretero R, Almagro M et al. (2009) Changes in activatory and inhibitory natural killer (NK) receptors may induce progression to multiple myeloma: implications for tumor evasion of T and NK cells. Hum Immunol 70: 854-857.

40. Romee R, Foley B, Lenvik T, Wang Y, Zhang B et al.(2013) NK cell CD16 surface expression and function is regulated by a disintegrin and metalloprotease-17 (ADAM17). Blood 121: 3599-3608.

41. Gasser S, Orsulic S, Brown EJ, Raulet DH (2005) The DNA damage pathway regulates innate immune system ligands of the NKG2D receptor. Nature 436: 1186-1190.

42. Bottino C, Castriconi R, Pende D, Rivera P, Nanni M et al. (2003) Identification of PVR (CD155) and Nectin-2 (CD112) as cell surface ligands for the human DNAM-1 (CD226) activating molecule. J Exp Med 198: 5575-67.

43. Baychelier F, Sennepin A, Ermonval M, Dorgham K, Debré P et al. (2013) Identification of a cellular ligand for the natural cytotoxicity receptor NKp44 Blood 122: 2935-2942.

44. Brandt CS, Baratin M, Yi EC, Kennedy J, Gao Z, Fox B et al.(2009) The B7 family member B7-H6 is a tumor cell ligand for the activating natural killer cell receptor NKp30 in humans. J Exp Med 206: 1495-1503.

45. Mandelboim O, Malik P, Davis DM, Jo CH, Boyson JE et al. (1999) Human CD16 as a lysis receptor mediating direct natural killer cell cytotoxicity. Proc Natl Acad Sci U S A. 96: 5640-5644.

46. Thielens A, Vivier E, Romagne F (2012) NK cell MHC class I specific receptors (KIR): from biology to clinical intervention. Curr Opin Immunol 24: 239-245.

47. Moretta A, Sivori S, Vitale M, Pende D, Morelli L et al. (1995) Existence of both inhibitory (p58) and activatory ( $p 50)$ receptors for HLA-C molecules in human natural killer cells. J Exp Med 182: 875-884.

48. Colonna M, Borsellino G, Falco M, Ferrara GB, Strominger JL et al. (1993) HLA-C is the inhibitory ligand that determines dominant resistance to lysis by NK1- and NK2-specific natural killer cells. Proc Natl Acad Sci U S A 90: 12000-12004. 
49. Thielens A, Vivier E, Romagne F (2012) NK cell MHC class I specific receptors (KIR): from biology to clinical intervention. Curr Opin Immunol 24: 239-245.

50. Braud VM, Allan DS, O'Callaghan CA, Söderström K, D'Andrea A et al. (1998) HLA-E binds to natural killer cell receptors CD94/NKG2A, B and C. Nature 391: 795-799.

51. Borrego F, Masilamani M, Kabat J, Sanni TB, Coligan JE (2005) The cell biology of the human natural killer cell CD94/NKG2A inhibitory receptor. Mol Immunol 42: 485-488.

52. Gunturi A, Berg RE, Forman J (2004) The role of CD94/NKG2 in innate and adaptive immunity. Immunol Res 30: 29-34.

53. Iwaszko M, Bogunia-Kubik K (2011) Clinical significance of the HLA-E and CD94/NKG2 interaction. Arch Immunol Ther Exp (Warsz) 59: 353-367.

54. Carrington M, Norman P (2003) The KIR Gene Cluster. National Center for Biotechnology Information.

55. Jonsson AH, Yokoyama WM (2009) Natural killer cell tolerance licensing and other mechanisms. Adv Immunol 101:27-79.

56. Kim S, Poursine-Laurent J, Truscott SM, Lybarger L, Song YJ et al. (2005) Licensing of natural killer cells by host major histocompatibility complex class I molecules. Nature 436: 709-713.

57. Santourlidis S, Trompeter HI, Weinhold S, Eisermann B, Meyer KL et al (2002) Crucial role of DNA methylation in determination of clonally distributed killer cell Ig-like receptor expression patterns in NK cells. J Immunol 169: 4253-4261.

58. Godfrey J, Benson DM Jr (2012) The role of natural killer cells in immunity against multiple myeloma. Leuk Lymphoma 53:1666-1676.

59. Manning LS, Chamberlain NL, Leahy MF, Cordingley FT (1995) Assessment of the therapeutic potential of cytokines, cytotoxic drugs and effector cell populations for the treatment of multiple myeloma using the 5 T33 murine myeloma model. Immunol Cell Biol 73: 326-332.

60. Manning LS, Berger JD, O'Donoghue HL, Sheridan GN, Claringbold PG, et al. (1992) A model of multiple myeloma: culture of 5T33 murine myeloma cells and evaluation of tumorigenicity in the C57BL/KaLwRij mouse. Br J Cancer 66: 1088-1093.

61. Azab AK, Hu J, Quang P, Azab F, Pitsillides C, et al. (2012) Hypoxia promotes dissemination of multiple myeloma through acquisition of epithelial to mesenchymal transition-like features. Blood 119: 5782-5794.

62. Alici E, Konstantinidis KV, Sutlu T, Aints A, Gahrton G, et al. (2007) Anti-myeloma activity of endogenous and adoptively transferred activated natural killer cells in experimental multiple myeloma model. Exp Hematol 35: 1839-1846.

63. Swift BE, Williams BA, Kosaka Y, Wang XH, Medin JA, et al. (2012) Natural killer cell lines preferentially kill clonogenic multiple myeloma cells and decrease myeloma engraftment in a bioluminescent xenograft mouse model. Haematologica 97: 1020-1028.

64. Garg TK, Szmania SM, Khan JA, Hoering A, Malbrough PA, et al. (2012) Highly activated and expanded natural killer cells for multiple myeloma immunotherapy. Haematologica 97: 1348-1356.

65. Lehmann D, Spanholtz J, OsI M, Tordoir M, Lipnik K, et al. (2012) Ex vivo generated natural killer cells acquire typical natural killer receptors and display a cytotoxic gene expression profile similar to peripheral blood natural killer cells. Stem Cells Dev 21: 2926-2938.

66. Spanholtz J, Tordoir M, Eissens D, Preijers F, van der Meer A, et al. (2010) High log-scale expansion of functional human natural killer cells from umbilical cord blood CD34-positive cells for adoptive cancer immunotherapy. PLoS One 5: e9221.

67. Berg M, Lundqvist A, McCoy P, Jr., Samsel L, Fan Y, et al. (2009) Clinical-grade ex vivo-expanded human natural killer cells up-regulate activating receptors and death receptor ligands and have enhanced cytolytic activity against tumor cells. Cytotherapy 11: 341-355.

68. Knorr DA, Bock A, Brentjens RJ, Kaufman DS (2013) Engineered human embryonic stem cell-derived lymphocytes to study in vivo trafficking and immunotherapy. Stem Cells Dev 22: 1861-1869.

69. Kang L, Voskinarian-Berse V, Law E, Reddin T, Bhatia M, et al. (2013) Characterization and ex vivo Expansion of Human Placenta-Derived Natural Killer Cells for Cancer Immunotherapy. Front Immunol 4: 101.

70. Shi J, Tricot G, Szmania S, Rosen N, Garg TK, et al. (2008) Infusion of haplo-identical killer immunoglobulin-like receptor ligand mismatched NK cells for relapsed myeloma in the setting of autologous stem cell transplantation. Br J Haematol 143: 641-653. 
71. Chew V, Toh HC, Abastado JP (2012) Immune microenvironment in tumor progression: characteristics and challenges for therapy. J Oncol 2012: 608406.

72. Matsumoto T, Abe M (2011) TGF-beta-related mechanisms of bone destruction in multiple myeloma. Bone 48: 129-134.

73. Wrzesinski SH, Wan YY, Flavell RA (2007) Transforming growth factor-beta and the immune response: implications for anticancer therapy. Clin Cancer Res 13: 5262-5270.

74. Wilson EB, El-Jawhari JJ, Neilson AL, Hall GD, Melcher AA, et al. (2011) Human tumour immune evasion via TGF-beta blocks NK cell activation but not survival allowing therapeutic restoration of anti-tumour activity. PLoS One 6: e22842.

75. Tanner J, Tosato G (1991) Impairment of natural killer functions by interleukin 6 increases lymphoblastoid cell tumorigenicity in athymic mice. J Clin Invest 88: 239-247.

76. Frassanito MA, Silvestris F, Cafforio P, Silvestris N, Dammacco F (1997) IgG M-components in active myeloma patients induce a down-regulation of natural killer cell activity. Int J Clin Lab Res 27: 48-54.

77. Jinushi M, Vanneman M, Munshi NC, Tai YT, Prabhala RH, et al. (2008) MHC class I chain-related protein A antibodies and shedding are associated with the progression of multiple myeloma. Proc Natl Acad Sci U S A 105: 1285-1290.

78. Lu ZY, Bataille R, Poubelle P, Rapp MJ, Harousseau JL et al. (1995) An interleukin 1 receptor antagonist blocks the IL-1-induced IL-6 paracrine production through a prostaglandin E2-related mechanism in multiple myeloma. Stem Cells 2: 28-34.

79. Van Elssen CH, Vanderlocht J, Oth T, Senden-Gijsbers BL, Germeraad WT et al. (2011) Inflammationrestraining effects of prostaglandin E2 on natural killer-dendritic cell (NK-DC) interaction are imprinted during DC maturation. Blood 118: 2473-2482.

80. Fauriat C, Mallet F, Olive D, Costello RT (2006) Impaired activating receptor expression pattern in natural killer cells from patients with multiple myeloma. Leukemia 20: 732-733.

81. Benson DM Jr, Bakan CE, Mishra A, Hofmeister CC, Efebera Y et al. (2010) The PD-1/PD-L1 axis modulates the natural killer cell versus multiple myeloma effect: a therapeutic target for CT-011, a novel monoclonal anti-PD-1 antibody. Blood 116: 2286-2294.

82. Soriani A, Zingoni A, Cerboni C, lannitto ML, Ricciardi MR et al. (2009) ATM-ATR-dependent upregulation of DNAM-1 and NKG2D ligands on multiple myeloma cells by therapeutic agents results in enhanced NK-cell susceptibility and is associated with a senescent phenotype. Blood 113: 3503-3511.

83. Carbone E, Neri P, Mesuraca M, Fulciniti MT, Otsuki T, et al. (2005) HLA class I, NKG2D, and natural cytotoxicity receptors regulate multiple myeloma cell recognition by natural killer cells. Blood 105 : 251-258.

84. Ryan HE, Poloni M, McNulty W, Elson D, Gassmann M et al. (2000) Hypoxia-inducible factor-1 alpha is a positive factor in solid tumor growth. Cancer Res 60: 4010-4015.

85. Hockel M, Vaupel P (2001) Tumor hypoxia: definitions and current clinical, biologic, and molecular aspects. J Natl Cancer Inst 93: 266-276.

86. Vaupel P, Thews O, Hoeckel M (2001) Treatment resistance of solid tumors: role of hypoxia and anemia. Med Oncol 18: 243-259.

87. Graeber TG, Osmanian C, Jacks T, Housman DE, Koch CJ et al. Hypoxia-mediated selection of cells with diminished apoptotic potential in solid tumors. Nature 379: 88-91.

88. Parmar K, Mauch P, Vergilio JA, Sackstein R, Down JD (2007) Distribution of hematopoietic stem cells in the bone marrow according to regional hypoxia. Proc Natl Acad Sci U S A 104: 5431-5436.

89. Hu J, Handisides DR, Van Valckenborgh E, De Raeve H, Menu E, Vande Broek I et al. 2010 Targeting the multiple myeloma hypoxic niche with TH-302, a hypoxia-activated prodrug. Blood 116: 1524-1527.

90. Giatromanolaki A, Bai M, Margaritis D, Bourantas KL, Koukourakis MI, et al. (2010) Hypoxia and activated VEGF/receptor pathway in multiple myeloma. Anticancer Res 30: 2831-2836.

91. Martin SK, Diamond P, Gronthos S, Peet DJ, Zannettino AC (2011) The emerging role of hypoxia, HIF-1 and HIF-2 in multiple myeloma. Leukemia 25: 1533-1542.

92. Colla S, Storti P, Donofrio G, Todoerti K, Bolzoni M, et al. (2010) Low bone marrow oxygen tension and hypoxia-inducible factor-1alpha overexpression characterize patients with multiple myeloma: role on the transcriptional and proangiogenic profiles of CD138(+) cells. Leukemia 24: 1967-1970. 


\section{Chapter}

Hypoxia induced impairment of NK cell cytotoxicity against Multiple Myeloma can be overcome by IL-2 activation of the NK cells

Subhashis Sarkar, Wilfred T.V. Germeraad, Kasper M.A. Rouschop, Elisabeth M.P. Steeghs, Michel van Gelder, Gerard M.J. Bos, Lotte Wieten

PLoS One.2013;8:e64835 


\section{Abstract}

\section{Background}

Multiple Myeloma (MM) is an incurable plasma cell malignancy residing within the bone marrow (BM). We aim to develop allogeneic Natural Killer (NK) cell immunotherapy for MM. As the BM contains hypoxic regions and the tumor environment can be immunosuppressive, we hypothesized that hypoxia inhibits NK cell anti-MM responses.

\section{Methods}

NK cells were isolated from healthy donors by negative selection and NK cell function and phenotype were examined at oxygen levels representative of hypoxic BM using flowcytometry. Additionally, NK cells were activated with IL-2 to enhance NK cell cytotoxicity under hypoxia.

\section{Results}

Hypoxia reduced NK cell killing of MM cell lines in an oxygen dependent manner. Under hypoxia, NK cells maintained their ability to degranulate in response to target cells, though, the percentage of degranulating NK cells was slightly reduced. Adaptation of NK- or MM cells to hypoxia was not required; hence, the oxygen level during the killing process was critical. Hypoxia did not alter surface expression of NK cell ligands (HLA-ABC, -E, MICA/B and ULBP1-2) and receptors (KIR, NKG2A/C, DNAM-1, NCRs and 2B4). It did, however, decrease expression of the activating NKG2D receptor and of intracellular perforin and granzyme B. Pre-activation of NK cells by IL-2 abrogated the detrimental effects of hypoxia and increased NKG2D expression. This emphasized that activated NK cells can mediate anti-MM effects, even under hypoxic conditions.

\section{Conclusions}

Hypoxia abolishes the killing potential of NK cells against Multiple Myeloma, which can be restored by IL-2 activation. Our study shows that for the design of NK cell-based immunotherapy it is necessary to study biological interactions between NK- and tumor cells also under hypoxic conditions. 


\section{Introduction}

Multiple Myeloma (MM) is an incurable plasma cell malignancy that is usually localized within the bone marrow $(\mathrm{BM})^{1}$. Cellular immunotherapy could be an interesting novel treatment option for MM. Natural Killer (NK) cell based therapies are of special interest since NK cells and even alloreactive NK cells, can be given to patients without causing graft versus host disease (GvHD). This is in contrast to alloreactive T cells and therefore an important benefit above the procedures focusing on T cell activity. In in vitro MM models, NK cells, both autologous and allogeneic, have been demonstrated to effectively eliminate MM cells ${ }^{2,3}$. Furthermore, NK cells isolated from myeloma patients have been expanded with $500 \mathrm{IU} / \mathrm{ml} \mathrm{IL-2}$ and shown to have ex vivo killing potential against autologous tumor cells ${ }^{4}$. Moreover, patient derived NK cells have been primed ex vivo with the tumor cell line CTV-1a resulting in improved killing of autologous and allogeneic MM cells ${ }^{5}$. Garg et al. have demonstrated expansion of patient derived NK cells by $\mathrm{K} 562$ cells transfected with 41BBL and membrane-bound interleukin-15 in the presence of $300 \mathrm{U} / \mathrm{ml} \mathrm{IL}^{2} 2^{6}$. More importantly, these expanded NK cells reduced myeloma burden in immunodeficient mice, and expanded in vivo in an IL-2 dependent fashion. Benson et al. has demonstrated that NK cells derived from MM patients express the inhibitory receptor PD-1 while NK cells from healthy individuals do not express this receptor unless activated by IL-2. They also show that blocking the interaction of the receptor and its ligand PD-L1 increases NK cell cytotoxicity against $\mathrm{MM}^{7}$. More recently, anti-KIR antibodies, with the scope of mimicking a KIR-HLA mismatched alloreactive response, have been suggested to provide an alternative strategy to boost NK cell immunity ${ }^{8}$. A first clinical study has shown that administration of IL-2 activated haploidentical KIR ligand mismatched NK cells to MM patients was safe, and $50 \%$ of the patients had near complete remission ${ }^{3}$. Together these data show the potential of NK cells in MM and they emphasize that there is room for improvement of the response. Better understanding of the factors influencing effective NK cell anti-tumor responses can help to maximize NK cell anti-MM responses.

The tumor microenvironment can influence disease progression and response to therapy in cancer. Hypoxia is a prominent feature of the tumor microenvironment and considered an adverse prognostic factor best documented for solid tumors ${ }^{9}$. Hypoxia is also a physiological characteristic of the $\mathrm{BM}^{10}$ and, as shown in mice studies, extremely hypoxic niches are essential for regulating the maintenance and functioning of hematopoietic stem cells ${ }^{11,12}$. Several recent studies have demonstrated that MM displays features of hypoxia; in the 5T33M mouse MM model, myelomatous BM has been shown to be more hypoxic than normal BM. This was visualized by positive staining of MM BM, for both exogenous- (pimonidazole) and endogenous- (HIF-1 $\alpha$ ) markers of hypoxia, while normal BM stained only weakly positive $e^{13,14}$. In human BM aspirates, median oxygen tension did not clearly differ between controls and MM patients (around $55 \mathrm{mmHg}$ in all cohorts) ${ }^{15}$. By immunostaining of bone biopsies from 
the MM patients, this study also showed the accumulation of the hypoxia regulated factor HIF-1 $\alpha$ in MM BM, an observation that was in line with two other studies showing the expression of HIF-1 $\alpha$ in bone biopsies from MM patients ${ }^{16,17}$. The accumulation of HIF-1 $\alpha$ was indicative of the presence of hypoxic niches in the human BM.

It is now well known that hypoxia contributes to chemo- and radiotherapy resistance of tumor cells ${ }^{18}$. By contrast, our understanding on how hypoxia assists tumor cells in escaping from immune-surveillance is in its infancy, but, increased knowledge could help to make immunotherapy more effective. One reported mechanism of tumor cell escape is hypoxia-induced shedding- and decreased surface expression of MHC class I chain-related (MIC) molecules resulting in reduced cytotoxicity of IL-2 stimulated peripheral blood lymphocytes (PBL) against prostate cancer cells ${ }^{19,20}$. The impact of hypoxia on NK cell function has been examined in only a very limited number of studies; in a first study, mouse YAC-1 cells were lysed at $21 \%$ and $1 \%$ oxygen, but were moderately killed by NK cells at $0 \%$ oxygen ${ }^{21}$. By contrast, a second study described a decrease, at $2 \%$ and $1 \%$ of oxygen, in NK cell killing of the $\mathrm{K} 562$ cell line $\mathrm{e}^{22}$, the human MHC negative equivalent of mouse YAC-1. The latter study also showed a partial reduction of NK cell cytotoxicity against human liver tumor cell lines at low levels of oxygen. To investigate if hypoxia is an inhibitory factor for NK cell immunity against hematological cancers, these first, partially contradictory, findings need to be further investigated in HLA expressing hematological cells.

In the present study, we hypothesize that one of the biological reasons for limited clinical success of NK cell therapy is, that suppressive factors, like hypoxia, in the BM environment decrease NK cell anti-MM responses. To study this hypothesis, we aim to investigate the influence of hypoxia on NK cell anti-MM responses using in vitro approaches where oxygen levels are representative of the tumor micro-environment. Secondly, we are interested in the impact of hypoxia on NK cell activating and inhibitory receptors in attempt to unravel the mechanism at play, as a disturbance by hypoxia on these balancing signals could be limiting effective NK cell therapy.

\section{Materials and methods}

\section{Cell lines and culture systems}

K562 (obtained from the ATCC) and the myeloma cell lines RPMI-8226/S (obtained from ATCC), OPM-1 and L-363 (received from Dr. A. Martens, UMC Utrecht, The Netherlands) ${ }^{23}$ were cultured in RPMI-1640 medium (Gibco, Breda, The Netherlands) supplemented with $10 \%$ fetal calf serum (Integro, Zaandam, The Netherlands), $100 \mathrm{U} / \mathrm{ml}$ penicillin (Gibco) and $100 \mu \mathrm{g} / \mathrm{ml}$ streptomycin (Gibco) at $37^{\circ} \mathrm{C}$ in humidified air containing $5 \% \mathrm{CO}_{2}$. NK cells were isolated from buffy coats by density gradient 
centrifugation and negative selection of NK cells using MACS beads and columns according to manufacturer's protocol (Miltenyi Biotech, $\mathrm{GmbH}$, Bergisch Gladbach, Germany). The use of buffy coats, being a side product from an Medical Ethical Review Committee (METC) required procedure, does not need ethical approval in the Netherlands, under the Dutch Code for Proper Secondary Use of Human Tissue. These buffy coats were anonymous, and the individuals from whom the samples originated did not object to their use. MACS sorted human NK cells were cultured in RPMI-1640 medium (Gibco) supplemented with 10\% fetal calf serum (Integro), $100 \mathrm{U} / \mathrm{ml}$ penicillin (Gibco) and $100 \mathrm{\mu g} / \mathrm{ml}$ streptomycin (Gibco) at $37^{\circ} \mathrm{C}$ in humidified air containing $5 \% \mathrm{CO}_{2}$ and at $0 \% \mathrm{O}_{2}, 0.2 \% \mathrm{O}_{2}, 1 \% \mathrm{O}_{2}$ or $21 \% \mathrm{O}_{2}$. For experiments with IL-2 activation, NK cells were activated with $1000 \mathrm{IU} / \mathrm{ml}$ recombinant human IL-2 (Proleukin, Novartis) for 14-16 hours at $21 \% \mathrm{O}_{2}$ or $0 \% \mathrm{O}_{2}$. After 16 hours, the activated NK cells were resuspended in their culture plates and directly used for cytotoxicity and CD107a assays, without any wash step. In vitro hypoxia experiments were performed using a hypoxia chamber system at $0 \% \mathrm{O}_{2}$ (MG500-Don Whitley Scientific Ltd, UK), $0.2 \% \mathrm{O}_{2}$ and $1 \% \mathrm{O}_{2}$ (Invivo 2,1000 Ruskinn Technology Ltd, Bridgend, UK) at $37^{\circ} \mathrm{C}$ with $5 \% \mathrm{CO}_{2}$.

\section{Flowcytometry}

MM cell ligand expression on OPM-1 and NK cell receptor, perforin and granzyme B expression on freshly obtained NK cells was determined after 14-16 hours incubation at $21 \%$ or $0 \% \mathrm{O}_{2}$. For surface staining, cells were washed with FACS buffer (PBS, 1\% FCS) and stained for 30 minutes on ice in the dark. Intracellular staining (perforin/granzyme B) was performed using the cytofix/cytoperm kit (BD Pharmingen, Erembodegem, Belgium) according to manufacturer's protocol. Antibodies for MM cell ligands and NK cell receptors are listed in Table S2.1. Flow cytometric analyses were performed with BD FACS Canto II. Data were analyzed with FlowJo 7.6 or BD FACSDiva Software v6.1.2.

\section{Viability assay}

The PE-Annexin V Apoptosis Detection Kit I (BD Pharmingen) was used for detection of apoptotic NK cells. After 14 to 16 hours incubation at $21 \%$ or $0 \% \mathrm{O}_{2}$, cells were washed with PBS (Sigma-Aldrich, Zwijndrecht, the Netherlands) and stained with 7-AAD and Annexin V-PE. After 15 minutes incubation in the dark at room temperature, samples were analyzed with a BD FACS Canto II. Cell viability of MM was estimated at $21 \%$ or $0 \%$ $\mathrm{O}_{2}$ using propidium iodide $(\mathrm{PI})$.

\section{Cytotoxicity assay}

Cytotoxic potential of NK cells was determined in a 4.5 hour flow cytometry based assay. Effector NK cells were isolated from buffy coats of healthy individuals by negative selection on MACS columns (Miltenyi Biotech). Target cells were labeled with 
DiO (Sigma-Aldrich). Both effector and target cells were individually pre-incubated for 14-16 hours at $21 \%$ or $0-1 \% \mathrm{O}_{2}$ and subsequently combined in triplicate at $\mathrm{E}: \mathrm{T}$ ratios $5: 1$, 10:1 and 20:1 in Corning ${ }^{\circledR} \operatorname{Costar}^{\circledR}$ round-bottom 96 well plates. After 4.5 hours, cell death of DiO positive target cells was measured with PI and specific cytotoxicity was determined by the equation: (\% PI positive target cells - \% spontaneous PI positive cells) / (100 - \% spontaneous PI positive cells) $\times 100$.

\section{CD107a assay}

Degranulation of cytotoxic contents from NK cells was measured by analysis of the degranulation marker CD107a by flow cytometry ${ }^{24}$. MACS enriched NK cells and OPM-1 or $\mathrm{K} 562$ cells were individually pre-incubated for $14-16$ hours at $21 \%$ or $0 \% \mathrm{O}_{2}$ and thereafter combined at a 2:1 E:T ratio at either $21 \%$ or $0 \% \mathrm{O}_{2}$ for 4.5 hours. APC labeled anti-CD107a (clone: H4A3, BD Pharmingen) or the corresponding isotype was added to the wells within 5-10 minutes after combining NK and OPM-1 cells. As a positive control PMA (100 ng/ml, Sigma-Aldrich) and ionomycin ( $1 \mathrm{\mu g} / \mathrm{ml}$; Sigma-Aldrich) were added to the NK cells during the 4.5 hours degranulation assay.

\section{NKG2D blocking}

Anti-NKG2D antibody (clone 1D11) or matched IgG1 isotype control, both LEAF purified and obtained from Biolegend (San Diego, CA, USA), at a final concentration $10 \mu \mathrm{g} / \mathrm{ml}$ were added to NK cells 20 minutes before combining it with OPM-1 cells at E:T ratio 20:1 in a 4.5 hour cytotoxicity assay.

\section{Statistical analyses}

Statistics were performed with GraphPad Prism V (Graphpad Software Inc, San Diego, CA, USA). Effect of $0 \% \mathrm{O}_{2}$ on surface receptor expression was analyzed by paired $t$-test. Influence of hypoxia on NK cytotoxicity and degranulation was evaluated with repeated measures ANOVA with Bonferroni correction. ${ }^{*} P<0.05$ was considered statistically significant.

\section{Results}

\section{NK cell cytotoxicity towards MM cells is decreased during hypoxia}

Oxygen levels within solid tumors can be as low as $0 \% \mathrm{O}_{2}{ }^{25}$, and oxygen levels $\leq 1 \% \mathrm{O}_{2}$ have been reported in the bone marrow ${ }^{26}$ where $\mathrm{MM}$ cells reside ${ }^{15}$. Furthermore, high metabolic rates of proliferating MM cells presumably add to the creation of a hypoxic tumor microenvironment. As we intend to develop allogeneic NK cell therapy for this 
disease it is crucial to study NK cell mediated anti-MM responses at low levels of oxygen. We thus examined NK cell cytotoxicity by pre-incubating MM cell lines and NK cells at different oxygen levels, followed by assessment of cytotoxicity. This revealed that specific NK cell cytotoxicity was decreased at $1 \%, 0.2 \%$ and $0 \%$ compared to $21 \%$ $\mathrm{O}_{2}$ in all three of the tested MM cell lines (OPM-1, RPMI-8226/S and L-363) (Figure 2.1A-D). This observation was also confirmed at lower E:T ratio of 5:1 and 10:1 for the OPM-1 cell line (Figure S2.1). The decreased cytotoxicity under hypoxia was unrelated to NK cell viability because the percentage of viable NK cells (negative for Annexin V and 7AAD), after 16 hours of incubation at $0 \%$ or $21 \% \mathrm{O}_{2}$, was comparable (Figure S2.2A-B). In addition, hypoxia exposure did not induce death of MM cells (Figure S2.2C-D).

A

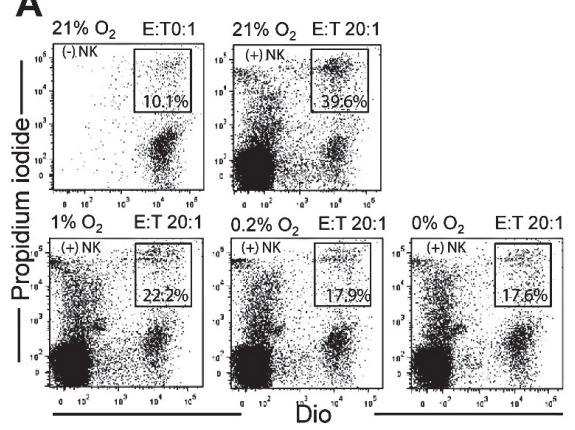

C

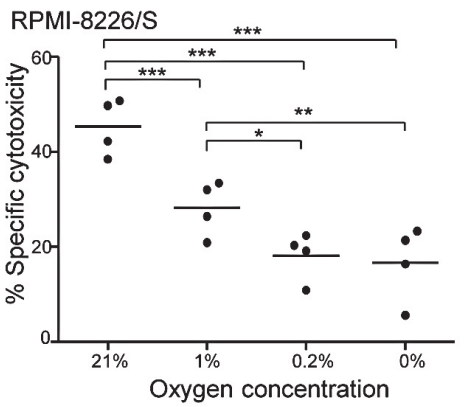

B

OPM-1

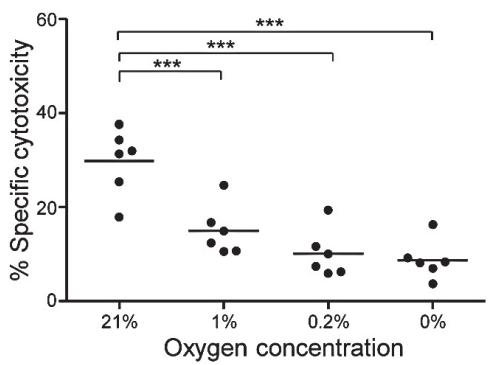

D

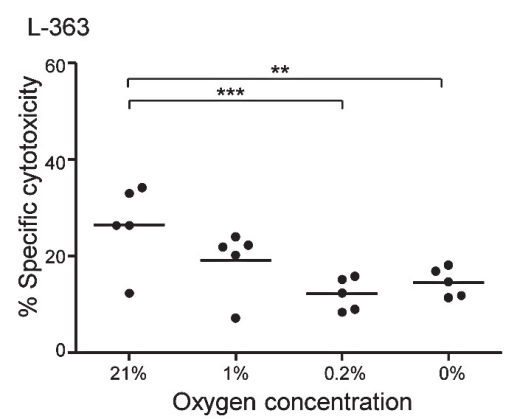

Figure 2.1 Hypoxia diminishes the cytotoxic potential of NK cells.

Freshly isolated NK cells and DiO labeled myeloma cell lines (OPM-1, RPMI-8226/S and L-363) were cultured at $21 \%, 1 \%, 0.2 \%$ or $0 \% \mathrm{O}_{2}$, for $14-16$ hours, followed by assessment of the cytotoxic potential of NK cells in a 4.5 hour assay at corresponding oxygen levels. (A) Representative dotplots of OPM-1 cells cultured with NK cells at 20:1 (E:T) ratio and (B-D) quantification of dotplots of 4-6 donors for different MM cell lines. Each dot represents mean of triplicate cultures of an individual donor. Statistics were performed with one-way repeated measures ANOVA with Bonferroni correction * $P<0.05$, ** $P<0.01, * * * P<0.001$. 


\section{Exposure to hypoxia decreases surface expression of NKG2D and CD16 on NK cells}

NK cell cytotoxicity is regulated by the balance of signaling through inhibitory and activating receptors ${ }^{27}$. As we found a decreased cytotoxicity at low oxygen tension, we examined if hypoxia induced phenotypic changes to NK cells or MM cells. For this, we studied the influence of hypoxia on surface expression of NK cell receptors. Expression levels of inhibitory receptors (KIRs and NKG2A), most activating receptors (NKG2C, NCRs, DNAM-1, and 2B4) and the adhesion molecule LFA-1 on NK cells were not influenced by hypoxia (Figure 2.2A and S2.3). By contrast, CD16 (Figure 2.2B) and intracellular perforin and granzyme B (Figure 2.2A and S2.4) expression was slightly but significantly decreased by hypoxia in all donors and NKG2D was lower in 11 of 12 analyzed donors (Figure 2.2B). NCR, DNAM-1 and NKG2D have been suggested to play a role in activation of NK cells by MM target cells ${ }^{2}$. To confirm that NKG2D was important in our system, we blocked the receptor using a specific antibody. The resulting decrease in killing of target OPM- 1 cells at $21 \% \mathrm{O}_{2}$ showed that NKG2D was indeed important for NK cell cytotoxicity against OPM-1 cells (Figure 2.2C). In addition to analysis of the receptors, we measured surface expression levels of several NK cell ligands on MM cells upon 16 hours of incubation at $21 \%$ and $0 \% \mathrm{O}_{2}$. HLA-E, the ligand for NKG2A, was not present on the surface of OPM-1 and RPMI-8226/S cells, whereas it was expressed at low levels by L-363 (Figure 2.3). HLA-ABC, the ligand for inhibitory KIRs was expressed at high levels by all three cell lines. Neither HLA-ABC nor HLA-E levels were clearly influenced by hypoxia. We also analyzed the influence of hypoxia on activating NKG2D ligands. Both OPM-1 and RPMI-8226/S cells expressed very low levels of MICA, intermediate levels of MICB and ULBP1 and somewhat higher levels of ULBP2, and the expression was not influenced by hypoxia. L-363 cells were negative for ULBP1 and had very low expression of MICA, MICB and ULBP2, which did not change upon incubation at low oxygen levels. For OPM-1, the hypoxia associated surface marker GLUT1 was enhanced upon incubation at $0 \% \mathrm{O}_{2}$. Another hypoxia associated marker, Carbonic anhydrase IX (CA IX) was upregulated on OPM-1 cells confirming the presence of hypoxia during the incubation. On RPMI-8266/S and L-363, GLUT1 and CAIX were not distinctly influenced by hypoxia. Overall, hypoxia had only a small effect on the phenotype of NK- and MM cells, but, possibly a relevant effect on NKG2D and CD16. 

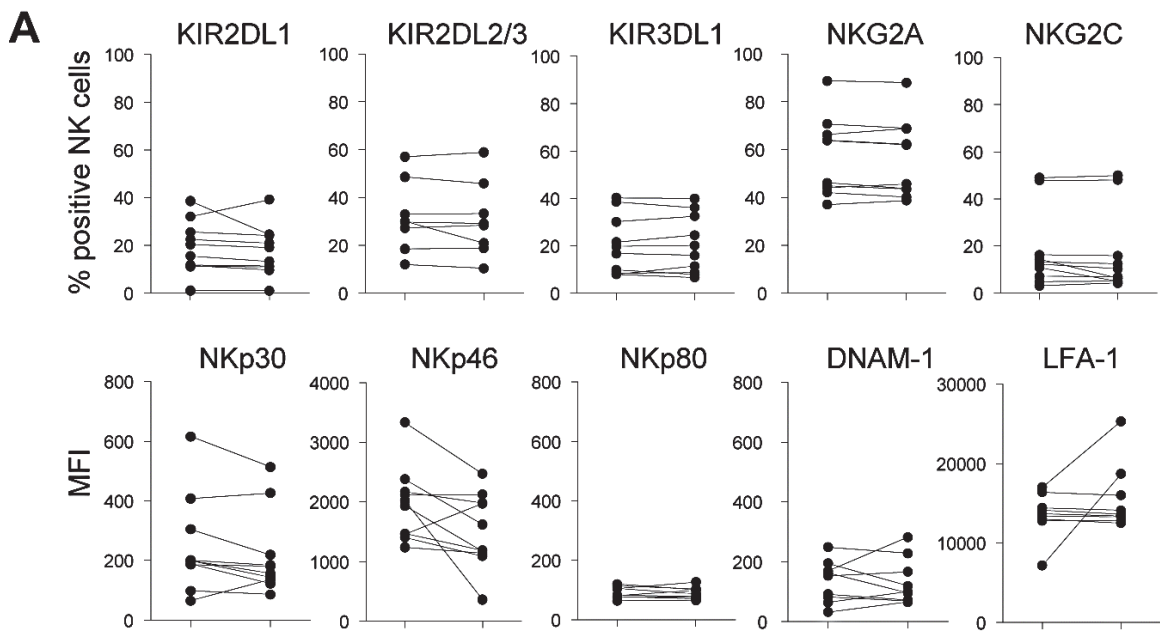

LFA-1
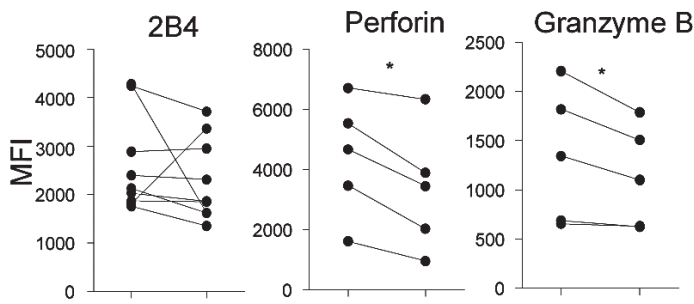

B
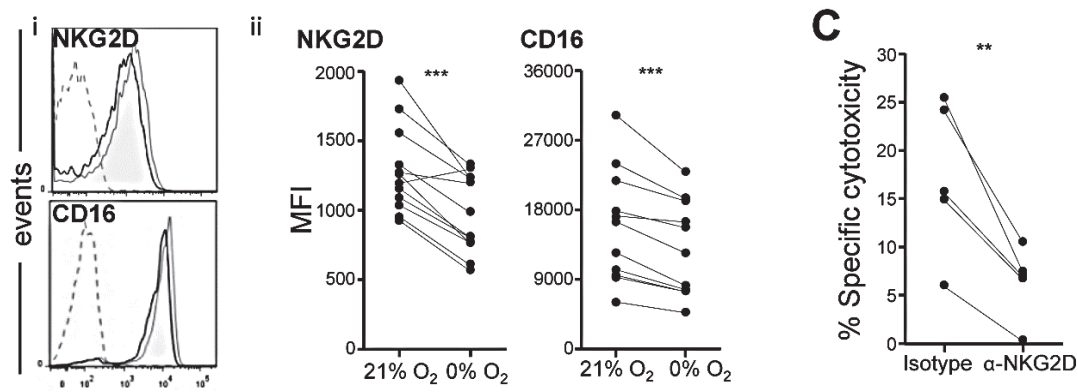

Figure 2.2 Hypoxia reduces surface expression of NKG2D and CD16 on NK cells and intracellular perforin and granzyme $B$.

(A) Flow cytometric analysis of surface expression of NK cell receptors and intracellular perforin and granzyme $\mathrm{B}$ after $14-16$ hours of incubation at $21 \%$ or $0 \% \mathrm{O}_{2}$. Graphs depict quantification of the \% positive NK cells or MFI for the different receptors. Each dot represents one donor. Shown alongside the graphs is the $x$-axis oxygen parameter for the graphs. (B) Flow cytometric analysis of surface expression of NKG2D $(n=12)$ and CD16 $(n=11)$ on NK cells after 14-16 hours of incubation at $21 \%$ or $0 \% \mathrm{O}_{2}$. (i) Representative histograms of surface expression at $21 \% \mathrm{O}_{2}$ (grey filled) or $0 \% \mathrm{O}_{2}$ (solid black line) and matched isotype control (dashed line) are displayed. (ii) Graphs represent quantification of the independent donors. (C) Cytotoxicity assay at E:T 20:1 in the presence of $10 \mu \mathrm{g} / \mathrm{ml} \alpha-N K G 2 D$ blocking antibody or matched isotype control. Each dot represents an individual donor. Statistics were performed with paired $t$-test. ** $P<0.01$ and $* * * P<0.001$ 

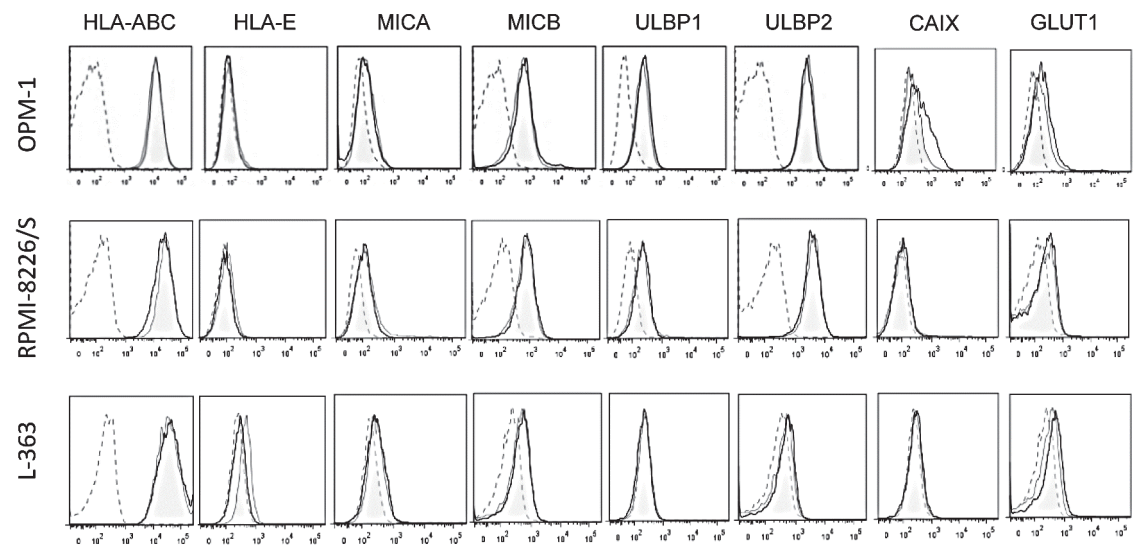

Figure 2.3 Hypoxia does not have an effect on surface expression of HLA-ABC and -E and NKG2D ligands. HLA-ABC, HLA-E and NKG2D ligands MICA/B and ULBP1-2 surface expression on OPM-1 target cells was analyzed after $14-16$ hours of incubation at $21 \% \mathrm{O}_{2}$ (grey filled) or $0 \% \mathrm{O}_{2}$ (solid black line). Matched isotype control is depicted by a dashed line. Expression of hypoxia associated hypoxia makers CAIX and GLUT1 was analyzed by flowcytometry after 14-16 hours incubation at $21 \% \mathrm{O}_{2}$ (grey filled) or $0 \% \mathrm{O}_{2}$ (solid black line). Matched isotype control is depicted by a dashed line.

\section{Adaptation of NK cells or MM cells to hypoxia is not required for the hypoxia-induced decrease in NK cell cytotoxicity}

To further investigate whether hypoxia-induced changes to either NK- or MM cells, or both, were responsible for the observed decrease in cytotoxicity, we pre-incubated NK cells, $\mathrm{MM}$ cells, or both at $21 \%$ or $0 \% \mathrm{O}_{2}$ and combined them for cytotoxicity assessment at $21 \%$ or $0 \% \mathrm{O}_{2}$. Pre-incubation of NK cells or OPM-1 cells at $0 \% \mathrm{O}_{2}$ did not influence NK cell cytotoxicity (Figure 2.4A; group 3 and 5). By contrast, all conditions where the cytotoxicity assay was performed at $0 \% \mathrm{O}_{2}$ (group 2, 4, 6 and 8), showed decreased NK cell cytotoxicity as compared to corresponding conditions with the cytotoxicity assay at $21 \% \mathrm{O}_{2}$ (Figure $2.4 \mathrm{~A}$; group $1,3,5$ and 7 ). Thus, the critical factor proved to be the $\mathrm{O}_{2}$ concentration during the 4.5 hour cytotoxicity assay, hence reintroducing $\mathrm{O}_{2}$ to tumor or NK cells cultured under hypoxic circumstances restored the cytotoxic effect almost immediately (for example group $7 \mathrm{vs.} \mathrm{8).} \mathrm{To} \mathrm{determine} \mathrm{if} \mathrm{this}$ could be explained by the hypoxia-induced decrease in NKG2D surface expression levels, we examined the kinetics of hypoxia-induced NKG2D downregulation. In line with the cytotoxicity assay described in Figure 2.4A, we first pre-incubated NK cells for 16 hours at either $21 \%$ or $0.2 \%$ of $\mathrm{O}_{2}$ and subsequently incubated them for another 4 hours at $21 \%$ ( $0.2 \%$ pre-incubated cells) or $0.2 \%$ ( $21 \%$ pre-incubated cells). For none of the donors the 4 hours of incubation at $0.2 \% \mathrm{O}_{2}$ reduced the NKG2D expression to the level observed after 16 hours of pre-incubation. Also, for the restoration of NKG2D levels, 4 hours at $21 \%$ was not sufficient (Figure 2.4B). 
A

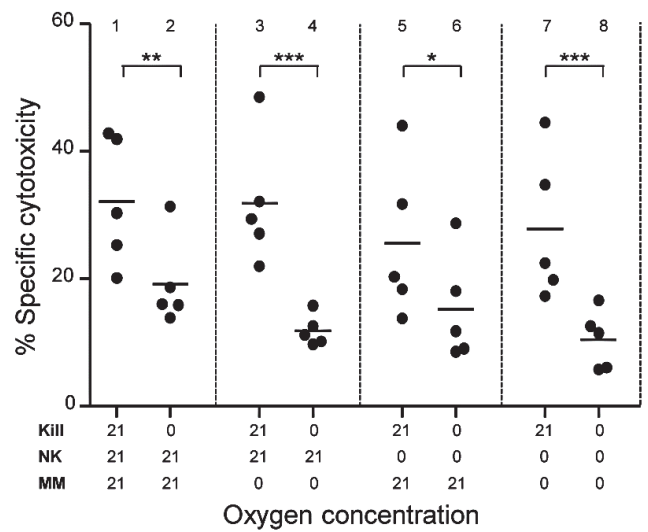

B

\begin{tabular}{|r|}
$-\cdots . \cdots-21 \%>0.2 \%$ \\
$-\quad 0.2 \% \rightarrow 21 \%$ \\
\hline
\end{tabular}
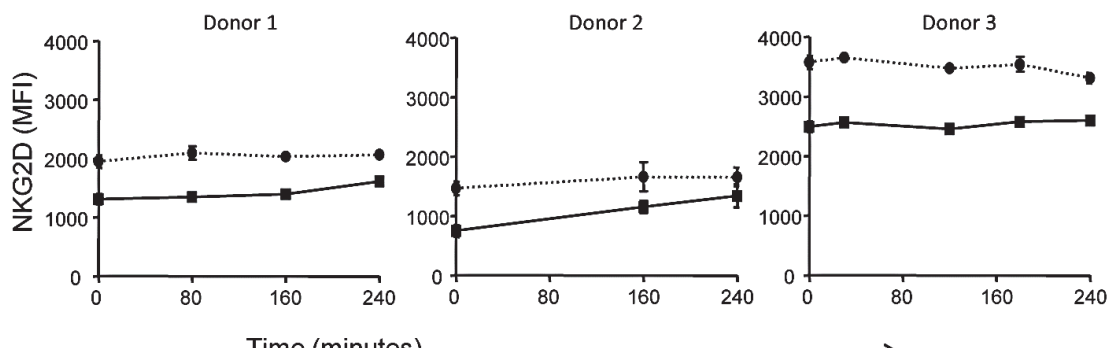

Time (minutes)

Figure 2.4 Oxygen concentration during NK cell killing is the key regulating parameter determining the cytotoxic potential.

(A) Pre-incubation (16 hours) of MM- and NK cells followed by 4.5 hour assessment of cytotoxicity were performed at the $\mathrm{O}_{2}$ concentration depicted on the $\mathrm{x}$-axis. Each dot represents mean of triplicate cultures of individual donors. Statistics were performed with one-way repeated measures ANOVA with Bonferroni correction * $P<0.05, * * P<0.01, * * * P<0.001$. (B) To determine the kinetics of NKG2D downregulation by hypoxia, NK cells were incubated for 16 hours at $21 \%$ or $0.2 \%$ oxygen. Thereafter, cells were transferred from $21 \%$ to $0.2 \%$ or from $0.2 \%$ to $21 \%$ oxygen. After the time indicated on the x-axis, NKG2D expression was measured by flowcytometry. Data show means +SD per donor of triplicate cultures.

\section{NK cell degranulation can occur under hypoxia but the percentage of degranulating NK cells is decreased}

As adaptation to hypoxia by NK or MM cells was not essential for the hypoxia-induced decrease in killing, we evaluated whether MM cells were able to induce NK cell degranulation under hypoxia. For this, we studied NK cell degranulation by analysis of the specific marker CD107a. We performed this in the absence of monensin to avoid interference with $\mathrm{pH}$ regulatory systems of the cell under hypoxia. NK cells did not 
vigorously degranulate in the absence of $\mathrm{MM}$ target cells. However, both at $21 \%$ and at $0 \%$ of $\mathrm{O}_{2}$, addition of OPM- 1 target cells to the NK cell cultures resulted in a significantly higher percentage of CD107a positive NK cells as compared to the corresponding condition without OPM-1 (Figure 2.5A,B). This demonstrated that NK cells maintained the ability to degranulate in response to OPM-1 under hypoxia. However, the percentage of degranulating NK cells in response to OPM-1 at $0 \% \mathrm{O}_{2}$ was slightly, but, significantly lower than the percentage of degranulating NK cells at $21 \% \mathrm{O}_{2}$ (Figure $2.5 \mathrm{~B}$ and S5). To investigate whether this decrease was caused by an intrinsic defect of NK cells to degranulate under hypoxic conditions we incubated NK cells with the universal control PMA/ionomycin or HLA-negative $\mathrm{K} 562$ cells at $21 \%$ or $0 \% \mathrm{O}_{2}$. This revealed that the percentage of CD107a positive NK cells upon PMA/ionomycin stimulation was comparable for $21 \%$ and $0 \% \mathrm{O}_{2}$ (Figure $5 \mathrm{~A}, \mathrm{C}$ ). Yet upon incubation with $\mathrm{K} 562$ cells, the percentage of degranulating $\mathrm{NK}$ cells at $0 \% \mathrm{O}_{2}$ was lower than the percentage of degranulating $\mathrm{NK}$ cells at $21 \% \mathrm{O}_{2}$ (Figure $2.5 \mathrm{C}$ and $\mathrm{S} 2.5$ ).

\section{NK cell activation by IL-2 can restore cytotoxicity against hypoxic MM cells}

In the context of NK cell immunotherapy, it is important to overcome the adverse effect of hypoxia. Strategies like targeting susceptibility of tumor cells or boosting NK cell cytotoxicity can be proposed. An interesting possibility is ex vivo pre-activation of NK cells. Since IL-2 is frequently being applied for clinical application after NK cell infusion $^{3,28,29}$, we used IL-2 $(1000 \mathrm{IU} / \mathrm{ml})$ to activate NK cells in vitro. NK cells activated with IL-2 at $21 \% \mathrm{O}_{2}$, had enhanced killing against OPM-1, both under normoxia and hypoxia (Figure 2.6A). Activation at $0 \% \mathrm{O}_{2}$ also boosted the killing potential of NK cells, however, to lesser extent than when activated at $21 \% \mathrm{O}_{2}$. IL-2 activation coincided with increased NK cell degranulation as shown by higher CD107a levels (Figure 2.6B and S2.6). That NK cells activated with IL-2 at $21 \% \mathrm{O}_{2}$ are potent at killing hypoxic myeloma cells was also observed for RPMI-8226/S and L-363 (Figure 2.6C). Thereby, our data, provided proof of concept that pre-activation of NK cells can overcome the inhibitory effects of hypoxia. Drug-induced upregulation of NK cell ligands like MICs and ULBPs is emerging as intervention strategy to improve anti-tumor effects of NK cells by enhancing sensitivity of tumor cells for NK cells ${ }^{30,31}$. To improve therapy, it could be interesting to combine these strategies with ex vivo or in vivo activation of NK cells. We therefore studied the influence of hypoxia on surface expression levels of receptors on IL-2 activated NK cells. This revealed that, hypoxia during IL-2 activation did not clearly influence surface expression levels of most NK cell receptors (Figure 2.6D). By contrast, intracellular perforin and granzyme B were lower expressed in NK cells activated by IL-2 at $0 \% \mathrm{O}_{2}$ as compared to NK cells activated at $21 \% \mathrm{O}_{2}$ (Figure $2.6 \mathrm{D}$ ). While IL-2 activation enhanced NKG2D expression levels at $21 \%$ and $0 \% \mathrm{O}_{2}$, the effect at $21 \%$ was more pronounced than at $0 \% \mathrm{O}_{2}(P=0.02)$ (Figure $\left.2.6 \mathrm{E}\right)$. 


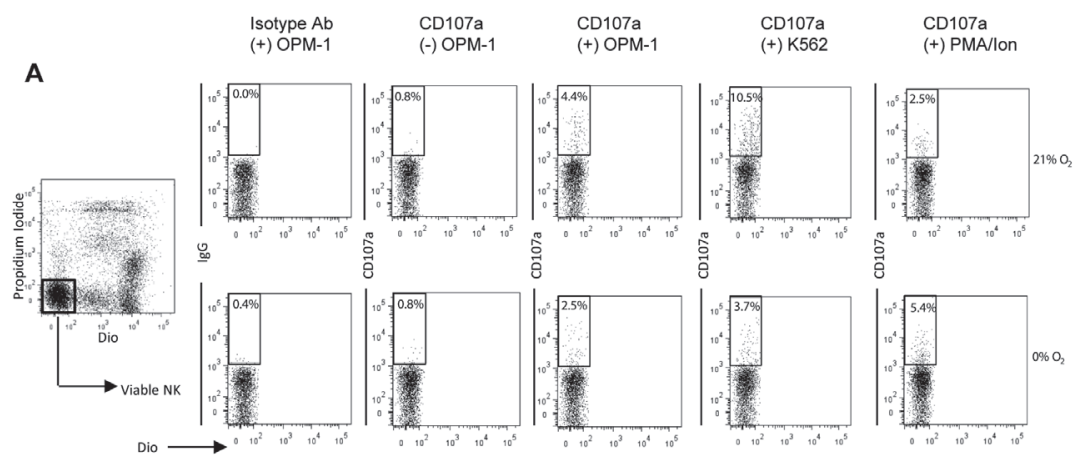

B

(+) OPM-1

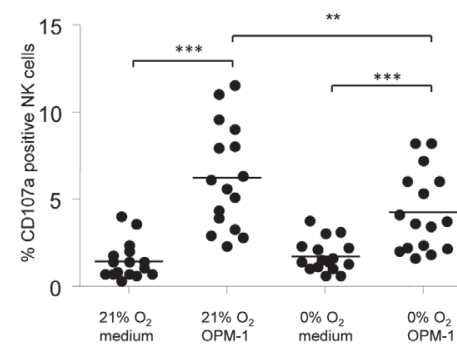

C

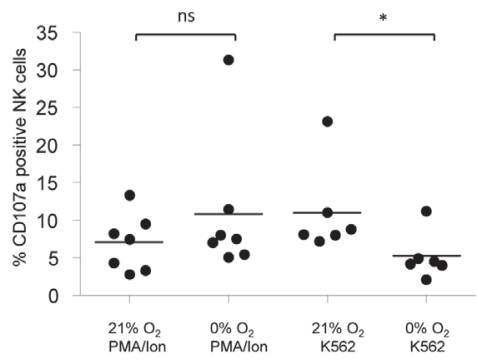

Figure 2.5 NK cell degranulation can occur under hypoxia but the percentage of degranulating NK cells is decreased.

NK cells and target cells (OPM- 1 or K562) were pre-incubated for $14-16$ hours at $21 \%$ or $0 \%$ of oxygen before they were combined in a 4.5 hour degranulation assay in the presence of an antibody specific for the degranulation marker CD107a or the corresponding isotype control. Percentage of CD107a positive NK cells for the isotype controls was always less than $0.5 \%$. To some of the cultures PMA/ionomycin was added during the 4.5 hour degranulation assay. The percentage of degranulated, CD107a positive NK cells was subsequently measured by flowcytometry. (A) Dot plots depicting data obtained for one representative donor. (B) Quantification of dot plots showing the percentage of CD107a positive NK cells upon incubation with medium or OPM-1 target cells. (C) Quantification of dot plots showing the percentage of CD107a positive NK cells upon incubation with PMA/ionomycin or K562 target cells. Each dot in $B$ and $C$ represents mean of duplicates of individual donors. Statistics were performed with one-way repeated measures ANOVA with Bonferroni correction * $P<0.05$, ** $P<0.01, * * * P<0.001$. 


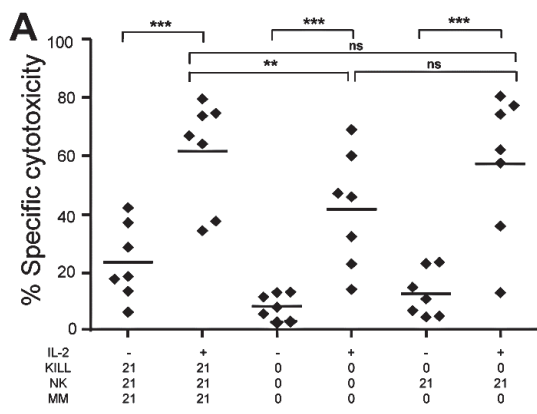

C RPMI-8226/S

L-363

B
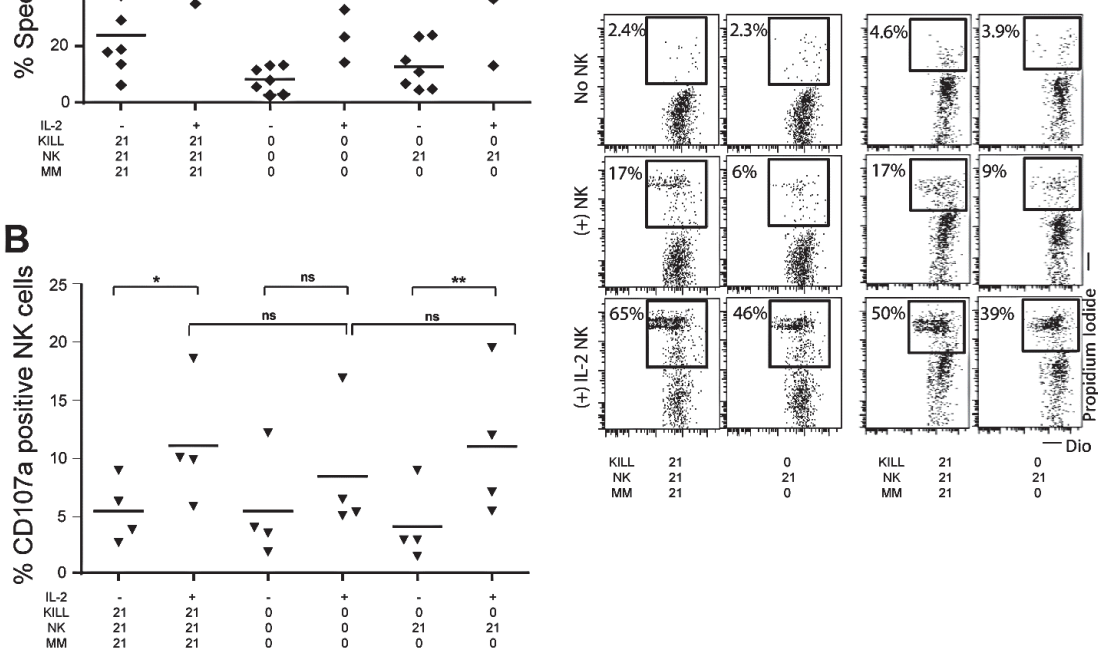

D
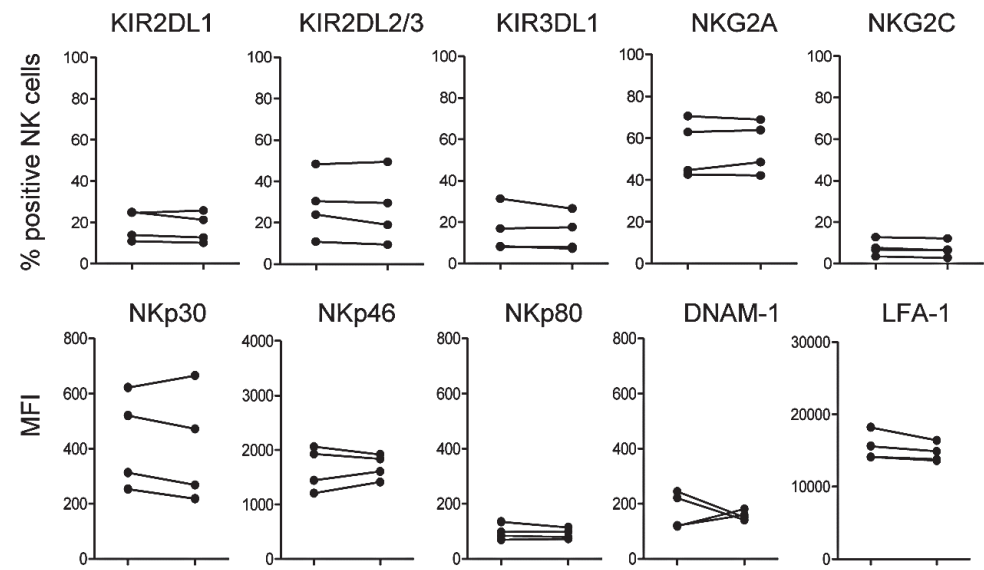

LFA-1
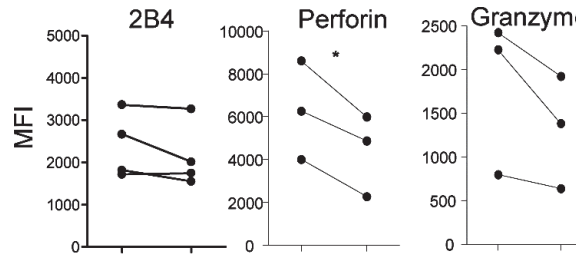

IL-2 activation

$21 \% \mathrm{O}_{2} \quad 0 \% \mathrm{O}_{2}$

$x$ - axis 
E
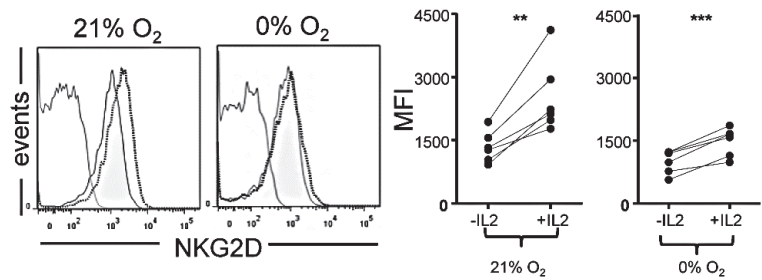

Figure 2.6 IL-2 activates NK cells as effector cells for targeting Multiple Myeloma in hypoxic environment.

(A-B) Pre-incubation (16h) of NK- and OPM-1 MM cells were performed as indicated on the $x$-axis. IL-2 activation of indicated conditions was done by adding $1000 \mathrm{IU} / \mathrm{ml}$ of IL-2 during the 16 hour pre-incubation period. Upon pre-incubation NK cells and MM cells were cocultured in a 4.5 hour cytotoxicty (A) or CD107a (B) assay. In (A) each dot represents mean of triplicate cultures of individual donors. In (B) NK cell degranulation was determined as described in figure 5 by quantification of the percentage of CD107a positive NK cells. Data in (B) show means of duplicate cultures of individual donors. Statistics were performed with one-way repeated measures ANOVA with Bonferroni correction * $P<0.05,{ }^{*} P<0.01, * * * P<0.001$. (C) NK cells and RPMI-8226/S or L-363 MM cells were pre-incubated as described in $6 \mathrm{~A}$ and subsequently combined in a 4.5 hour cytotoxicity assay. Plots show data obtained from one donor. (D) Flow cytometric analysis of surface expression of NK cell receptors and intracellular expression of perforin and granzyme $\mathrm{B}$ after $14-16$ hours of incubation at $21 \%$ or $0 \% \mathrm{O}_{2}$ in the presence of IL-2. Graphs depict quantification of the \% positive NK cells or MFI for the different receptors. Each dot represents one donor. Shown alongside the graphs is the $x$-axis oxygen parameter. (E) NKG2D surface expression on NK cells upon 14-16 hours incubation at $21 \% \mathrm{O}_{2}$ and $0 \% \mathrm{O}_{2}$ in the presence (solid black line) or absence (grey filled) of IL-2 and matched isotype (grey solid line). Each dot represents an individual donor. Statistics were performed with paired $t$-test. $* * p<0.01, * * * p<0.001$.

\section{Discussion}

During disease progression of $\mathrm{MM}$, the $\mathrm{BM}$ microenvironment combined with its stromal factors contributes to $\mathrm{MM}$ cell survival, outgrowth and drug resistance ${ }^{32}$. Insights into the mechanism of immunosuppression by individual tumor environmental factors can help to improve efficacy of immunotherapy. Despite the well-known presence of hypoxia in the microenvironment of tumors, our understanding of the impact of hypoxia on immune cell function is very limited. With the hypoxic signature in MM being confirmed in recent patient studies ${ }^{15,33}$, we now show that hypoxia is detrimental for NK cell mediated MM cell killing and a factor to consider when designing NK cell based immunotherapy.

In our study, we used a series of MHC class I expressing MM cell lines to more closely mimic the situation in patients because surface expression of MHC class I molecules is an important inhibitory factor for NK cells, and sensitivity of tumor cells for NK cells is regulated by MHC class I. The expression of MHC by our cell lines might also explain the 
relatively low levels of CD107a that we observed when analyzing NK cell degranulation. The two earlier studies that also used cell lines of hematological origin used MHC class I negative leukemia cell lines that are easy to kill targets, and are frequently being used as positive controls. One of these studies showed that $0 \% \mathrm{O}_{2}$ but not $1 \% \mathrm{O}_{2}$ reduced $\mathrm{NK}$ cell cytotoxicity against the mouse MHC negative tumor cell line YAC-1 ${ }^{21}$. By contrast, the second study demonstrated that $1 \% \mathrm{O}_{2}$ was sufficient to reduce NK cell cytotoxicity against $\mathrm{K562}$, the human equivalent of mouse YAC- $1^{22}$. Our data are in agreement with the latter study and provide first evidence that NK cytotoxicity against MHC positive $\mathrm{MM}$ is severely impaired at oxygen levels representative of the BM environment.

In the current study, we focused on MM, but since various other hematological tumors and several metastasized solid tumors also exploit the BM to survive and escape from therapy, our data can also be relevant for the response of these tumors to NK cell therapy. We used a series of established MM cell lines to investigate whether hypoxia is an inhibitory factor for effective NK cell anti-tumor responses. In a future study, it would be interesting to understand the influence of hypoxia on the interplay of tumor cells, NK cells and bone marrow stromal cells. Such studies with patient primary MM cells combined with our current knowledge on hypoxia restraining effect on primary NK cells would provide new insights on improving NK cell therapy.

To explore the mechanism of hypoxia mediated down regulation of NK cell cytotoxicity, we investigated, in parallel to NK cell cytotoxicity, the effect of hypoxia on activatingand inhibitory NK cell receptor expression. We observed that hypoxia lowered surface expression of NKG2D and CD16 on NK cells. Previously, hypoxia has been shown to increase tumor cell secretion of MICA that, upon binding, provoked a decrease in NKG2D surface expression ${ }^{19,20}$. In our experimental set up it seems however unlikely that the decrease in NKG2D expression could be explained through hypoxia induced MICA secretion by the tumor cells. The reduction in NKG2D was namely observed in the absence of $\mathrm{MM}$ cells and could be reversed by placing hypoxic NK cells at $21 \% \mathrm{O}_{2}$. Parkhurst et al. have recently reported less functional circulating NK cells, with lower expression of NKG2D and CD16 in melanoma and renal cell carcinoma patients post autologous NK cell infusion ${ }^{34}$. Also in breast cancer patients, decreased expression of activating receptors on NK cells isolated from the tumor has been reported ${ }^{35}$. These patient studies illustrate that the tumor environment can modulate the NK cell phenotype. Although a future study is required to unravel the underlying mechanism, our results provide direct evidence that hypoxia is a tumor environmental factor contributing to the down regulation of key-activating receptors like NKG2D.

In agreement with a previous study ${ }^{2}$, we showed that NKG2D is important for in vitro killing of MM targets since blocking of this receptor reduced NK cell killing of OPM-1. However, the hypoxia-induced decrease in NKG2D expression was small and our kinetics studies revealed that at least 4 hours of hypoxia were required to reduce surface expression levels. Furthermore, we demonstrated that the oxygen concentration during the killing process was the limiting and critical factor, indicating 
that the effect was rapid. Together this suggests that NKG2D was probably not the major factor explaining the decrease in cytotoxicity in our experimental model. We also observed a small reduction in CD16 expression on hypoxia incubated NK cells. CD16 is important for antibody-mediated cytotoxicity of NK cells. Since our experimental set up did not include MM specific antibodies, it seems unlikely that CD16 is mediating the decrease in cytotoxicity.

Another inhibitory factor could be the secretion of soluble molecules. In MM patients, MICA shedding has been associated with disease progression ${ }^{36}$. Furthermore, interfering with the hypoxia-induced accumulation of HIF-1 $\alpha$ and ADAM10 has been shown to enhance MICA surface expression and to reduce MICA shedding, leading to augmented cytotoxicity of PBL against DU145 prostate cancer cells under hypoxia ${ }^{19}$. In our study, we did not observe a change in surface expression of MICA/B or ULBP1-2 under hypoxia on any of the three MM cell lines we tested while CAIX and GLUT1, surrogate markers for hypoxia, were elevated on OPM-1 at $0 \% \mathrm{O}_{2}$. These markers were not clearly enhanced by hypoxia on RPMI-8226/S and L-363, which might result from differences in regulation or sensitivity between the cell lines. We did not rule out that MICs and ULBPs were shed while maintaining a stable surface expression on the tumor cell. But, addition of supernatant obtained from hypoxic OPM-1 cells to $21 \% \mathrm{O}_{2}$ cultures did not clearly influence NK cell cytotoxicity in our system (data not shown). This suggested that these soluble mediators were not the major factor for the NK cell response against OPM-1. The inhibitory effect of shedding of soluble NKG2D ligands in response to hypoxia is mainly known for MICA. Since OPM-1 expressed low levels of MICA, it is possible that, in contrast to the prostate cancer cells, MICA is not shed by OPM-1 cells. This illustrates that the secretion of soluble NKG2D ligands is cell type specific. Hence, to conclude that hypoxia induced soluble MICA does not play a role in $\mathrm{MM}$ in general, a cell line with higher surface expression of MICA will be required.

To investigate whether deficient degranulation could explain the decreased cytotoxicty under hypoxia, we used an experimental set up that was comparable to the setup of our cytotoxicity assay. In this set up we observed relatively low percentages of CD107a positive NK cells as compared to a previous study by Alter et al. This might be explained by the shorter incubation time that we used ( 4.5 hours) or by the 16 hours preincubation of the NK cells. Nevertheless, we provide evidence that NK cells were able to degranulate in response to OPM-1 cells under hypoxia, which indicated that triggering and activation of the NK cells was not completely abolished under hypoxia. However, we did observe a small but significant decrease in the percentage of degranulating NK cells and in the amount of intracellular perforin and granzyme B. The decrease in degranulation was not caused by an intrinsic defect of NK cells to degranulate under hypoxia as incubation of NK cells with a chemical positive control, PMA/ionomycin, did yield comparable levels of degranulation at $21 \%$ and $0 \% \mathrm{O}_{2}$. In response to the universal positive control cell line $\mathrm{K} 562$ we did detect decreased $\mathrm{NK}$ cell degranulation at $0 \% \mathrm{O}_{2}$ as compared to $21 \% \mathrm{O}_{2}$. Therefore, it seems likely that the reduction in degranulation 
occurred in a target cell line dependent manner. In a previous study, CD8 T cell mediated killing of tumor cells has been shown to be impaired by hypoxia ${ }^{37}$. These authors confirmed that the cytotoxic machinery of CD8 T cells was functional at low oxygen levels and that the decrease in killing could be explained by HIF-1 $\alpha$ mediated resistance of the tumor cells. Since we showed that no long-term adaptation of NK- or MM cells was required, it will be interesting to study the contribution of the factors described for CD8 T cells to NK cell anti-tumor responses in an hypoxic environment.

Hypoxia can contribute to the development of chemoresistance. Incomplete chemotherapeutic elimination of tumor cells can be detrimental for the patient and could lead to disease relapse ${ }^{38}$. We recently showed in a mouse model that alloreactive NK cells can cure mice from established 4T1 breast cancer and that this includes the eradication of the population of chemo resistant cells ${ }^{39}$. It would be interesting to investigate whether a similar strategy could help to eliminate chemo resistant MM cells. Alloreactive (KIR-HLA mismatched) NK cells will probably represent the most potent source of NK cells as these subsets will receive a minimal amount of inhibitory signals due to the KIR-HLA mismatch. Alternatively anti-KIR antibodies ${ }^{8}$ could help creating a similar effect. Moreover, targeting residual tumor cells within their hypoxic niche with appropriately activated NK cells can be an approach to eradicate tumors.

Together, our data strongly advocates performing any further in vitro investigation to study interactions between immune cells and hematological tumor cells - at least MM also under hypoxic conditions. Furthermore, our study provides proof of concept that activating cytokines can overcome the adverse effects of hypoxia in vitro and shows that hypoxia is a factor to take into account when designing allogeneic NK cell based immunotherapy for MM. Experimental set ups comparable to ours will be helpful to determine the potential of novel and existing cytokines or immunomodulatory agents, to boost NK cell responses in a way that NK cells can also exert their effector function in the presence of tumor environmental factors like hypoxia. For future clinical perspectives it might be considered to combine NK cell therapy with hypoxia-targeting and pre-activation of NK cells to eliminate tumor cells in a hypoxic environment.

\section{Acknowledgements}

The authors would like to thank Dr. Joris Vanderlocht (MUMC+) for critical discussion and Dr. Anton Martens for OPM-1 and L-363 cell lines. 


\section{REFERENCES}

1. Delorme S, Baur-Melnyk A (2009) Imaging in Multiple Myeloma. Eur J Radiol 70: 401-408.

2. Carbone E, Neri P, Mesuraca M, Fulciniti MT, Otsuki T, et al. (2005) HLA class I, NKG2D, and natural cytotoxicity receptors regulate multiple myeloma cell recognition by natural killer cells. Blood 105: 251-258.

3. Shi J, Tricot G, Szmania S, Rosen N, Garg TK, et al. (2008) Infusion of haplo-identical killer immunoglobulin-like receptor ligand mismatched NK cells for relapsed myeloma in the setting of autologous stem cell transplantation. Br J Haematol 143: 641-653.

4. Alici E, Sutlu T, Björkstrand B, Gilljam M, Stellan B, et al. (2008) Autologous antitumor activity by NK cells expanded from myeloma patients using GMP-compliant components. Blood 111: 3155-3162.

5. Katodritou E, Terpos E, North J, Kottaridis P, Verrou E, et al. (2011) Tumor-primed natural killer cells from patients with multiple myeloma lyse autologous, NK-resistant, bone marrow-derived malignant plasma cells. Am J Hematol 86: 967-973.

6. Garg TK, Szmania SM, Khan JA, Hoering A, Malbrough PA, et al. (2012) Highly activated and expanded natural killer cells for multiple myeloma immunotherapy. Haematologica 97: 1348-1356.

7. Benson DM Jr, Bakan CE, Mishra A, Hofmeister CC, Efebera Y, et al. (2010) The PD-1/PD-L1 axis modulates the natural killer cell versus multiple myeloma effect: a therapeutic target for CT-011, a novel monoclonal anti-PD-1 antibody. Blood 116: 2286-2294.

8. Benson DM Jr, Hofmeister CC, Padmanabhan S, Suvannasankha A, Jagannath S, et al. (2012) A phase 1 trial of the anti-KIR antibody IPH2101 in patients with relapsed/refractory multiple myeloma. Blood 120: 4324-4333.

9. Harris AL (2002) Hypoxia-A key regulatory factor in tumor growth. Nat Rev Cancer 2: 38-47.

10. Watanabe Y, Terashima Y, Takenaka N, Kobayashi M, Matsushita T (2007) Prediction of avascular necrosis of the femoral head by measuring intramedullary oxygen tension after femoral neck fracture. J Orthop Trauma 21: 456-461.

11. Parmar K, Mauch P, Vergilio JA, Sackstein R, Down JD (2007) Distribution of hematopoietic stem cells in the bone marrow according to regional hypoxia. Proc Natl Acad Sci USA 104: 5431-5436.

12. Rehn M, Olsson A, Reckzeh K, Diffner E, Carmeliet $P$, et al. (2011) Hypoxic induction of vascular endothelial growth factor regulates murine hematopoietic stem cell function in the low-oxygenic niche. Blood 118: 1534-1543.

13. Azab AK, Hu J, Quang P, Azab F, Pitsillides C, et al. (2012) Hypoxia promotes dissemination of multiple myeloma through acquisition of epithelial to mesenchymal transition-like features. Blood 119: 5782-5794.

14. Hu J, Handisides DR, Van Valckenborgh E, De Raeve H, Menu E, et al. (2010) Targeting the multiple myeloma hypoxic niche with TH-302, a hypoxia-activated prodrug. Blood 116: 1524-1527.

15. Colla S, Storti P, Donofrio G, Todoerti K, Bolzoni M, et al. (2010) Low bone marrow oxygen tension and hypoxia-inducible factor- $1 \alpha$ overexpression characterize patients with multiple myeloma: role on the transcriptional and proangiogenic profiles of CD138(+) cells. Leukemia 24: 1967-1970.

16. Giatromanolaki A, Bai M, Margaritis D, Bourantas KL, Koukourakis MI, et al. (2010) Hypoxia and activated VEGF/receptor pathway in multiple myeloma. Anticancer Res 30: 2831-2836.

17. Martin SK, Diamond P, Williams SA, To LB, Peet DJ, et al. (2010) Hypoxia-inducible factor-2 is a novel regulator of aberrant CXCL12 expression in multiple myeloma plasma cells. Haematologica 95: 776-784.

18. Rohwer N, Cramer T (2011) Hypoxia-mediated drug resistance: novel insights on the functional interaction of HIFs and cell death pathways. Drug Resist Updat 14: 191-201.

19. Barsoum IB, Hamilton TK, Li X, Cotechini T, Miles EA, et al. (2011) Hypoxia induces escape from innate immunity in cancer cells via increased expression of ADAM10: role of nitric oxide. Cancer Res 71: 7433-7441.

20. Siemens DR, Hu N, Sheikhi AK, Chung E, Frederiksen L, et al. (2008) Hypoxia increases tumor cell shedding of MHC class I chain-related molecule: role of nitric oxide. Cancer Res 68: 4746-4753.

21. Loeffler DA, Juneau PL, Heppner GH (1991) Natural killer-cell activity under conditions reflective of tumor micro-environment. Int J Cancer 48: 895-899. 
22. Fink T, Ebbesen P, Koppelhus U, Zachar V (2003) Natural killer cell-mediated basal and interferonenhanced cytotoxicity against liver cancer cells is significantly impaired under in vivo oxygen conditions. Scand J Immunol 58: 607-612.

23. Rozemuller H, van der Spek E, Bogers-Boer LH, Zwart MC, Verweij V, et al. (2008) A bioluminescence imaging based in vivo model for preclinical testing of novel cellular immunotherapy strategies to improve the graft-versus-myeloma effect. Haematologica 93: 1049-1057.

24. Alter G, Malenfant JM, Altfeld M (2004) CD107a as a functional marker for the identification of natural killer cell activity. J Immunol Methods 294: 15-22.

25. Helmlinger G, Yuan F, Dellian M, Jain RK (1997) Interstitial pH and pO2 gradients in solid tumors in vivo: high-resolution measurements reveal a lack of correlation. Nat Med 3: 177-182.

26. Guitart AV, Hammoud M, Dello Sbarba P, Ivanovic Z, Praloran V (2010) Slow-cycling/quiescence balance of hematopoietic stem cells is related to physiological gradient of oxygen. Exp Hematol 38: 847-851.

27. Ljunggren HG, Malmberg KJ (2007) Prospects for the use of NK cells in immunotherapy of human cancer. Nat Rev Immunol 7: 329-339.

28. Rubnitz JE, Inaba H, Ribeiro RC, Pounds S, Rooney B, et al. (2010) NKAML: a pilot study to determine the safety and feasibility of haploidentical natural killer cell transplantation in childhood acute myeloid leukemia. J Clin Oncol 28: 955-959.

29. Sutlu T, Alici E (2009) Natural killer cell-based immunotherapy in cancer: current insights and future prospects. J Intern Med 266: 154-181.

30. Chávez-Blanco A, De la Cruz-Hernández E, Domínguez GI, Rodríguez-Cortez O, Alatorre B, et al. (2011) Upregulation of NKG2D ligands and enhanced natural killer cell cytotoxicity by hydralazine and valproate. Int J Oncol 39: 1491-1499.

31. Nausch N, Cerwenka A (2008) NKG2D ligands in tumor immunity. Oncogene 27: 5944-5958.

32. Xu S, Menu E, De Becker A, Van Camp B, Vanderkerken K, et al. (2012) Bone marrow-derived mesenchymal stromal cells are attracted by multiple myeloma cell-produced chemokine CCL25 and favor myeloma cell growth in vitro and in vivo. Stem Cells 30: 266-279.

33. Martin SK, Diamond P, Gronthos S, Peet DJ, Zannettino AC (2011) The emerging role of hypoxia, HIF-1 and HIF-2 in multiple myeloma. Leukemia 25: 1533-1542.

34. Parkhurst MR, Riley JP, Dudley ME, Rosenberg SA (2011) Adoptive transfer of autologous natural killer cells leads to high levels of circulating natural killer cells but does not mediate tumor regression. Clin Cancer Res 17: 6287-6297.

35. Mamessier E, Sylvain A, Bertucci F, Castellano R, Finetti P, et al. (2011) Human breast tumor cells induce self-tolerance mechanisms to avoid NKG2D-mediated and DNAM-mediated NK cell recognition. Cancer Res 71: 6621-6632.

36. Jinushi M, Vanneman M, Munshi NC, Tai YT, Prabhala RH, et al. (2008) MHC class I chain-related protein A antibodies and shedding are associated with the progression of multiple myeloma. Proc Natl Acad Sci USA 105: 1285-1290.

37. Noman MZ, Buart S, Van Pelt J, Richon C, Hasmim M, et al. (2009) The cooperative induction of hypoxiainducible factor-1 alpha and STAT3 during hypoxia induced an impairment of tumor susceptibility to CTL-mediated cell lysis. J Immunol 182: 3510-3521.

38. Matsunaga T, Imataki O, Torii E, Kameda T, Shide K, et al. (2012) Elevated HIF-1 $\alpha$ expression of acute myelogenous leukemia stem cells in the endosteal hypoxic zone may be a cause of minimal residual disease in bone marrow after chemotherapy. Leuk Res 36: 122-124.

39. Frings PW, Van Elssen CH, Wieten L, Matos C, Hupperets PS, et al. (2011) Elimination of the chemotherapy resistant subpopulation of 4T1 mouse breast cancer by haploidentical NK cells cures the vast majority of mice. Breast Cancer Res Treat 130: 773-781. 


\section{Supporting information}

A

E:T 5:1

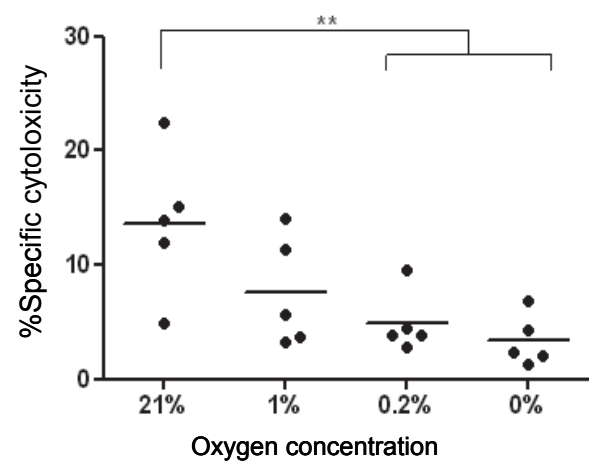

B E:T 10:1

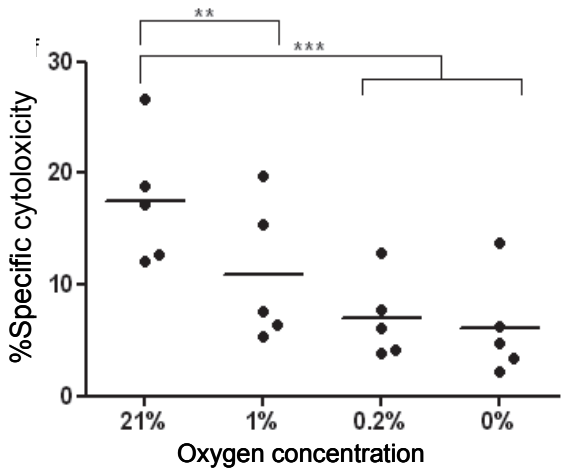

Figure S2.1 NK cell cytotoxicity for OPM-1 is suppressed in an hypoxic environment at E:T ratios of 5:1 and 10:1.

Both Multiple Myeloma and NK cells were pre-incubated at different oxygen concentrations and combined in E:T ratios of (A) 5:1 and (B) 10:1 in 4.5 hour kill assay. Cytotoxicity was estimated by flow cytometry. Statistics in the figure were performed as: ${ }^{*} P<0.05$ with One-Way repeated measures ANOVA with Bonferroni correction. Each dot represents mean of triplicates for independent donors $(n=5)$. 
A

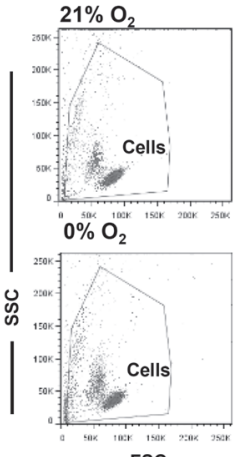

B

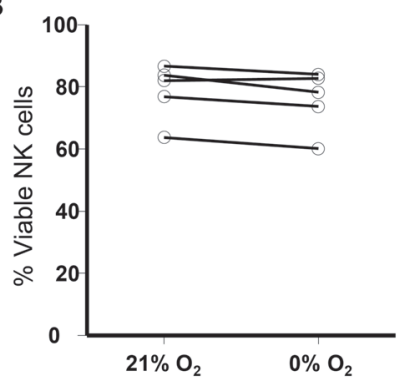

C

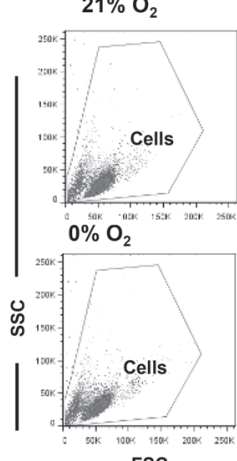

$\mathrm{D}$

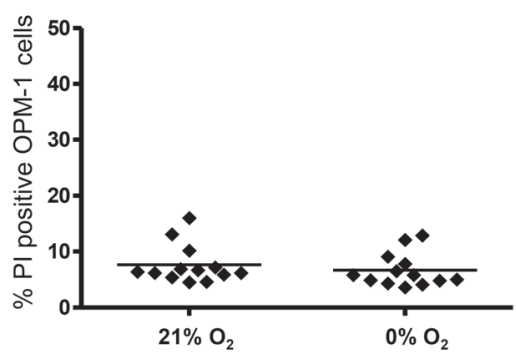

Figure S2.2 Hypoxia does not increase cell death of NK and OPM-1 cells.

(A) Viability of NK cells under different oxygen concentrations was estimated. NK cells were gated on FSC vs. SSC to exclude debris. Percentage of early and late apoptotic cells as estimated by the sum of single 7-AAD+, single Annexin $V+$ and double 7-AAD+Annexin $V+$ cells. (B) Percentage of viable NK cells was determined as $100-\%$ (single 7-AAD+, single Annexin $V+$ and double 7-AAD+Annexin V+ cells) ( $n=5)$. (C) OPM-1 cells were incubated for 16 hours at $21 \%$ or $0 \% \mathrm{O}_{2}$ followed by Annexin $\mathrm{V}$ - 7AAD apoptosis staining. (D) Spontaneous cell death of OPM-1 as estimated by propidium iodide in independent kill assays consistently ranged between $\sim 6-10 \%$ $(n=13)$. 


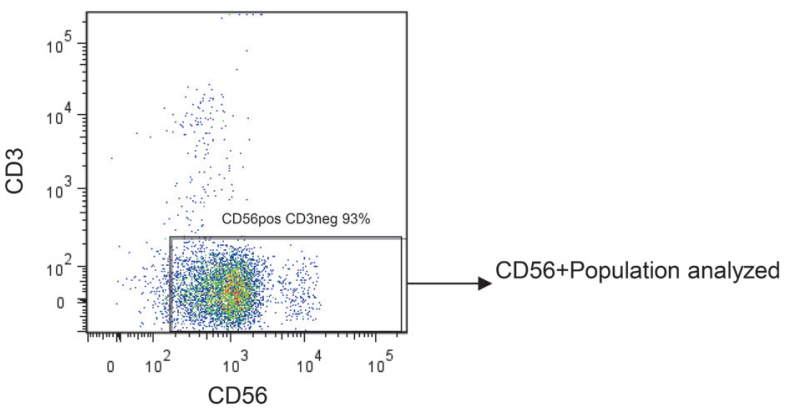

A
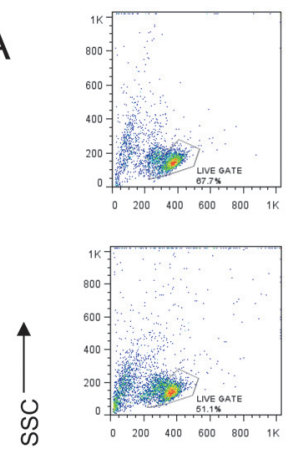

FSC
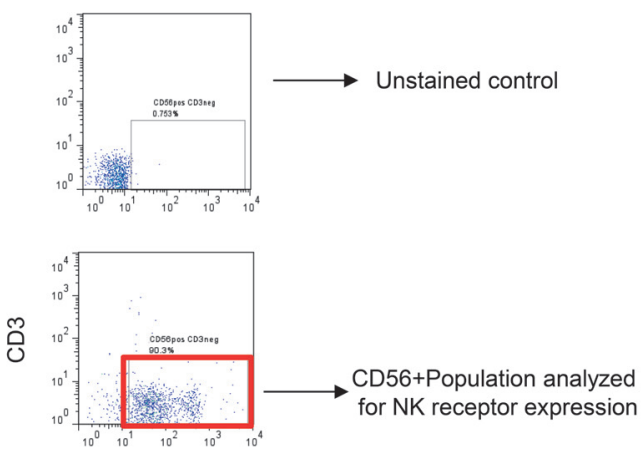

CD56

B
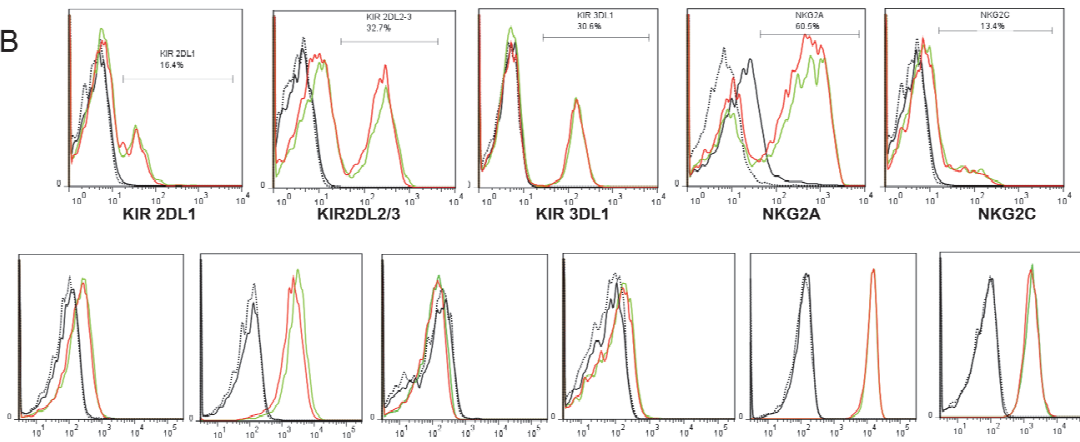

NKp30
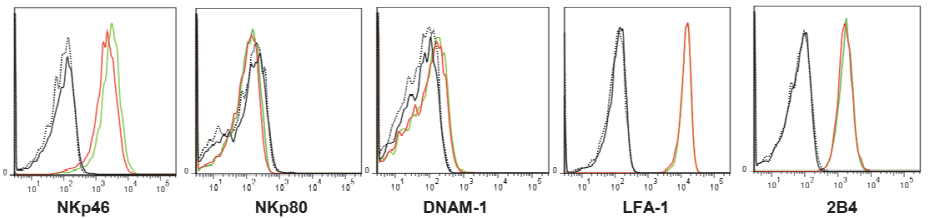

Figure S2.3 Flowcytometry analysis of NK cell receptors at $21 \%$ and $0 \%$ Oxygen.

Flow cytometric analysis of surface expression of NK cell receptors after 14-16 hours of incubation at $21 \%$ or $0 \% \mathrm{O}_{2}$. (A) MACS sorted NK cells were analyzed by flowcytometry. Cells with $\mathrm{FSC}^{\text {high }}$ were selected for analysis. These cells were $>90 \%$ pure for CD56 (bottom figure). This population was downstream analyzed for NK receptors in figure B. (B) Histogram plots of NK cell receptors. In each plot, isotype controls at $21 \% \mathrm{O}_{2}$ (black bold) or $0 \% \mathrm{O}_{2}$ (black dotted) have been plotted against the respective receptor at $21 \% \mathrm{O}_{2}$ (green) or $0 \% \mathrm{O}_{2}$ (red). The percentage of NK cells positive for KIR2DL1, KIR2DL2/3, KIR3DL1, NKG2A and NKG2C are shown against relevant isotype control. The mean fluorescence intensity of the NK cell receptors NKp30, NKp46, NKp80, DNAM-1, LFA-1 and 2B4 have been described in Figure 2.2A. The data shown is complete analysis for one individual donor. 

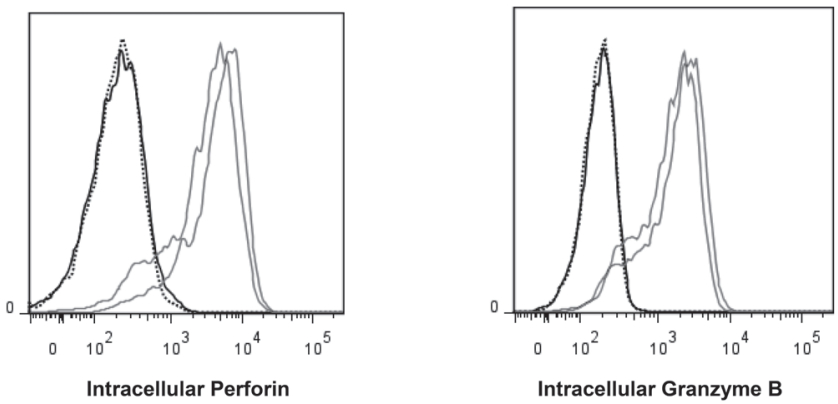

Figure S2.4 Flowcytometry analysis of intracellular Perforin and Granzyme B at $\mathbf{2 1 \%}$ and 0\% Oxygen.

Flow cytometric analysis of intracellular Perforin and Granzyme B expression within NK cells after $14-16$ hours of incubation at $21 \%$ or $0 \% \mathrm{O}_{2}$. MACS sorted NK cells were analyzed by flowcytometry. The cells were $>90 \%$ pure for CD56. This population was downstream analyzed for Perforin and Granzyme B expression. Histogram plots representing isotype controls at $21 \%$ $\mathrm{O}_{2}$ (black bold) or $0 \% \mathrm{O}_{2}$ (black dotted) have been plotted against the respective intracellular contents at $21 \% \mathrm{O}_{2}$ (green) or $0 \% \mathrm{O}_{2}$ (red).

A $\quad(+)$ OPM-1

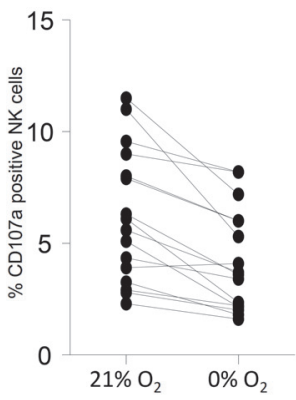

(+) PMA/Ion

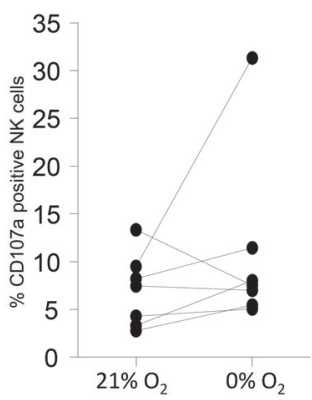

C $\quad(+) \mathrm{K} 562$

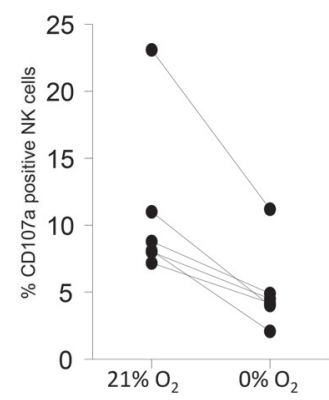

Figure S2.5 Percentage of degranulating NK cells is decreased hypoxia.

NK cells and target cells (OPM-1 or K562) were preincubated for $14-16$ hours at $21 \%$ or $0 \%$ of oxygen. After this, they were combined in a 4.5 hour degranulation assay in the presence of CD107a. To some of the cultures PMA/lonomycin was added during the 4.5 hours degranulation assay. Data shown here represented paired analysis of CD107a expression on NK cells, in response to (A) OPM-1 (B) PMA/lonomycin and (C) K562. The data represented in this figure are the same data as depicted in figure 2.5. Each dot is the mean \% of CD107a of duplicate cultures of one donor. Lines connect data obtained at $21 \%$ and $0 \%$ of oxygen for one donor. 


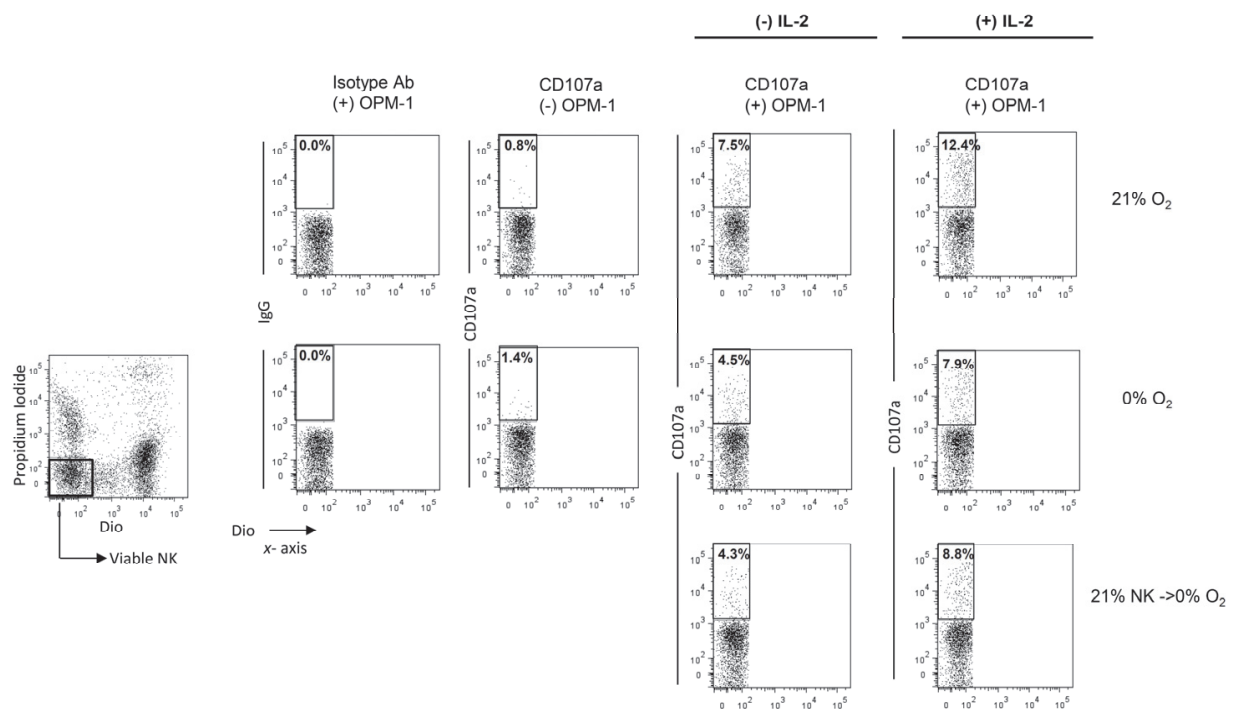

Figure S2.6 IL-2 activation increases CD107a expression on NK cells both $21 \%$ and $0 \%$ Oxygen.

NK- and OPM-1 MM cells were preincubated for $14-16$ hours at $21 \%$ or $0 \%$ of oxygen. During this 14-16 hours, NK cells were either plated in complete media as described in methods and materials, or supplemented additionally with $1000 \mathrm{IU} / \mathrm{ml}$ of IL-2. Upon pre-incubation NK cells and MM cells were cocultured in a 4.5 hours CD107a degranulation assay. Degranulation assay was performed either at $21 \%$ or $0 \%$ of oxygen. Dot plots in this figure are representative of complete analysis for one individual donor, for the phenomenon described in Figure 2.6B. 
Table S2.1 List of antlbodies

\begin{tabular}{llll}
\hline Name & Dye & Clone & Company \\
\hline HLA-E & PE & 3D12HLA-E & eBioscience \\
HLA-ABC & APC & G46-2.6 & BD Pharmingen \\
MICA & PE & 159227 & R\&D SYSTEMS \\
MICB & APC & 2356511 & R\&D SYSTEMS \\
ULBP1 & PE & 170818 & R\&D SYSTEMS \\
ULBP2 & APC & 165903 & R\&D SYSTEMS \\
CD3 & APC H7 & SK7 & BD Pharmingen \\
CD16 & FITC & $3 G 8$ & BD Pharmingen \\
CD56 & Horizon V460 & B159 & BD Pharmingen \\
DNAM-1 & APC & 102511 & R\&D SYSTEMS \\
KIR2DL1 & PE & 14311 & R\&D SYSTEMS \\
KIR2DL2/3 & APC & DX27 & Miltenyi Biotec \\
KIR3DL1 & FITC & DX9 & Miltenyi Biotec \\
LFA1 & FITC & HI111 & BD Pharmingen \\
NKG2A & PE-Cy5.5 & Z199 & Beckman Coultier Inc. \\
NKG2C & PE & 134591 & R\&D SYSTEMS \\
NKG2D & APC & BAT221 & Miltenyi Biotec \\
NKp30 & PE & 210845 & R\&D SYSTEMS \\
NKp46 & APC & SE2/NKp46 & BD Pharmingen \\
NKp80 & APC & 239127 & R\&D SYSTEMS \\
2B4 & PE & Polycl. & R\&D SYSTEMS \\
Perforin & PE & SG9 & BD Pharmingen \\
Granzyme B & FITC & GB11 & BD Pharmingen \\
GLUT1 & APC & 202915 & R\&D SYSTEMS \\
CAIX & FITC & 303123 & R\&D SYSTEMS \\
\hline
\end{tabular}




\section{Chapter}

\section{Optimal selection of Natural Killer cells to}

kill myeloma: the role of HLA-E

and NKG2A

Subhashis Sarkar, Michel van Gelder, Willy Noort, Yunping Xu, Kasper M.A. Rouschop, Richard Groen, Harry C. Schouten, Marcel G.J. Tilanus, Wilfred T.V. Germeraad, Anton C.M. Martens, Gerard M.J. Bos, Lotte Wieten

Submitted 


\section{Abstract}

Immunotherapy with allogeneic Natural Killer (NK) cells offers therapeutic perspectives for Multiple Myeloma patients. Here, we aimed to refine NK cell therapy by evaluation of the relevance of HLA-class I and HLA-E for NK-anti-myeloma reactivity. We show that HLA-class I was strongly expressed on the surface of patient-derived myeloma cells and of myeloma cell lines. HLA-E was highly expressed by primary myeloma cells but only marginally by cell lines. HLA-Elow expression on U266 cells observed in vitro was strongly upregulated after in vivo (bone marrow) growth in RAG-2-/- $\mathrm{cc}-/$-mice suggesting that in vitro HLA-E levels poorly predict for the in vivo situation. Concurrent analysis of inhibitory receptors (KIR2DL1, KIR2DL2/3, KIR3DL1 and NKG2A) and NK cell degranulation upon co-culture with myeloma cells revealed that KIR-ligand mismatched NK cells degranulate more than matched subsets, and that HLA-E abrogates degranulation of NKG2A+ subsets. Inhibition by HLA-class I and HLA-E was also observed with IL-2 activated NK cells and at low oxygen levels $(0.6 \%)$ mimicking hypoxic bone marrow niches where myeloma cells preferentially reside. Our study demonstrates that NKG2A-negative, KIR-ligand mismatched NK cells are the most potent subset for clinical application. We envision that infusion of high numbers of this subclass will enhance clinical efficacy. 


\section{Introduction}

Multiple Myeloma is caused by expansion of malignant plasma cells in the bone marrow (BM) and remains largely incurable with the current treatment options ${ }^{1}$. Allogeneic hematopoietic stem cell transplantation (allo-HSCT) performed over HLA-C barriers can provide curative anti-myeloma responses ${ }^{2}$. However, poor clinical outcomes and T cell mediated graft-versus-host disease (GvHD) are major limitations of allo-HSCT for myeloma ${ }^{3-6}$. Infusion of alloreactive Natural Killer cells (NK) cells could be an appealing strategy to improve clinical effectiveness since the risk of GvHD is low and there is no need for myeloablative conditioning ${ }^{7,8}$.

NK cells are large granular lymphocytes with the potential to selectively kill malignant cells. The balance between tolerance to healthy cells and eradication of virally-infected or tumor cells is established by a panel of activating and inhibitory receptors on the NK cell surface ${ }^{9}$. Activating receptors, interacting with "stress-induced flags" on diseased cells, trigger NK cell killing. Inhibitory receptors bind to self-molecules like HLA-class I and HLA-E that are expressed by virtually every normal cell and protect against unwanted NK cell reactivity. An important class of inhibitory receptors, the killer immunoglobulin-like receptors (KIRs), interacts with HLA-class I molecules on the target cell; KIR2DL1 ligates HLA-C group 2 (Lsy80) alleles, KIR2DL2/3 ligates HLA-C group 1 (Asn80) alleles and KIR3DL1 interacts with HLA-Bw4 motifs ${ }^{10,11}$. The lectin-like family member NKG2A interacts with HLA-E ${ }^{12}$.

Upon HLA-mismatched allo-HSCT, donor NK cells eliminate tumor cells when the patient lacks HLA-epitopes from the donor and donor NK cells express KIRs specific for these epitopes (KIR-ligand mismatch) ${ }^{13}$. Landmark studies in the haplo-identical HSCT setting showed that KIR-ligand mismatched NK cells exert anti-leukemic effects and improve survival of acute myeloid leukemia (AML) patients ${ }^{14,15}$. Also in myeloma, incompatibility between donor KIRs and patient HLA has been associated with a better clinical outcome ${ }^{2}$. Moreover, a first clinical trial showed that isolated KIR-ligand mismatched NK cells can be safely administrated to myeloma patients though the contribution of NK cells, in addition to the conditioning regime, could not be established in this trial ${ }^{16}$. Beneficial effects of KIR-ligand mismatched NK cell infusions have been observed in other types of cancer as well ${ }^{17-20}$. Although these studies are encouraging, optimization of the clinical efficacy of transferred NK cells is still required. While there are several reasons why NK cells therapy could be a major step forward in immunotherapy of cancer, various mechanisms have been described that could reduce clinical effectiveness; e.g. decreased expression of activating receptors ${ }^{21-23}$, hypoxia ${ }^{24}$, the expression of immunosuppressive soluble- or surface-molecules ${ }^{25-27}$ or infusion of insufficient numbers of (the right) NK cells and HLA-class I expression by myeloma cells $^{28-30}$. Genotypic selection of a KIR-ligand mismatched donor seems to be a good strategy to reduce inhibitory effects by HLA-class I. However, because NK cells can express one or a combination of inhibitory receptors, not all donor NK cells will 
exclusively express KIRs that are mismatched with HLA-class I of the patient. Furthermore, of all peripheral blood NK cells $20-80 \%$ expresses NKG2A ${ }^{31,32}$. For clinical grade expanded NK cells, either from hematopoietic progenitor cells ${ }^{33}$, blood derived NK cells ${ }^{34}$, or pluripotent stem cells ${ }^{35}$, this percentage can be higher than $80 \%$. Because HLA-E is low polymorphic and all functional HLA-E alleles can inhibit NK cells through NKG2A ${ }^{36}$, NKG2A could abolish the overall NK cell response against HLA-E expressing tumors, even in the KIR-ligand mismatched setting. This makes HLA-E clinically highly relevant, but information on HLA-E expression and its inhibitory potential in myeloma is lacking.

In the present study, aimed to refine the selection process for NK cell therapy, we studied the relevance of HLA-class I and HLA-E for anti-myeloma reactivity of allogeneic NK cells. First, we determined the presence of HLA-class I and HLA-E on primary myeloma cells and on myeloma cell lines, revealing that primary myeloma cells express both HLA-class I and HLA-E. Also, we found major differences with myeloma cells cultured in vitro and the same cells grown in vivo. Second, we assessed the inhibitory potential of HLA-class I and HLA-E on different NK cell subsets in a co-culture system that allowed synchronized analysis of degranulation of individual NK cells subsets demonstrating the potent inhibitory effects of HLA-class I and HLA-E on NK cell alloreactivity. To better predict for the in vivo response, we performed these assays at ambient- and clinically relevant low oxygen levels.

\section{Materials and methods}

\section{Cell culture incubators}

Experiments were performed in ambient $95 \%$ humidified air, $21 \% \mathrm{O}_{2}$ incubator (SANYO) containing $5 \% \mathrm{CO}_{2}$. Hypoxia experiments were completely performed inside $0.6 \% \mathrm{O}_{2}$ incubators (Invivo 2,1000 Ruskinn Technology Ltd) at $37^{\circ} \mathrm{C}$ with $5 \% \mathrm{CO}_{2}$.

\section{Cell lines}

Myeloma cell lines ${ }^{37}$ and HLA-class I deficient K562 cell line (ATCC) were cultured in RPMI-1640 medium (Gibco) supplemented with $10 \%$ fetal calf serum (Integro), $1000 \mathrm{U} / \mathrm{ml}$ penicillin (Gibco) and $100 \mu \mathrm{g} / \mathrm{ml}$ streptomycin (Gibco). The LME-1 cell line (received through Dr. R. van Oers, AMC, Amsterdam) was cultured in IMDM medium (Gibco), and XG-1 in IMDM medium with $(500 \mathrm{pg} / \mathrm{ml}$ ) IL-6 (Biosource).

\section{Flowcytometric analysis of HLA-E and HLA-class I expression}

Material from myeloma and plasma cell leukemia patients was obtained as a waste product from a diagnostic procedure that had been approved by the hospital's medical 
ethical committee. Bone marrow cells were stained for CD38 (HB7, BD), CD138 (CLB1D4, Sanquin), cytoplasmic Kappa light chain (G20-193, BD), cytoplasmic Lamba light chain (1-555-2, BD), HLA-class I (G46-2.6, BD), or HLA-E (3D12, eBioscience). HLA expression was determined on $\mathrm{CD} 38^{\text {high }}$ cells. All flowcytometry based assays were performed on a BD FACS Canto II. Data were analyzed with BD FACSDiva v6.1.2 or FlowJo 7.6.

\section{HLA genotyping and NK cell donor selection}

Genotypic expression of HLA epitopes (HLA-C1, -C2 and -Bw4) in cell lines (Table S3.1) and healthy volunteers was determined by Sequence-Specific Oligonucleotides (SSO) analysis and Luminex ${ }^{\circledR}$ according to the manufacturer's guidelines (One Lambda). Healthy volunteers, HLA-C1+C2+Bw4+ and phenotypically expressing KIR2DL1, KIR2DL2/3, and KIR3DL1, were selected as NK cell donors. All donors signed informed consent forms.

\section{NK cell isolation and CD107a degranulation assay}

NK cells were isolated from blood by negative MACS selection (Miltenyi Biotec) according to manufactures instructions. Immediately after isolation, NK cells were activated with $1000 \mathrm{U} / \mathrm{ml} \mathrm{IL-2} \mathrm{(Proleukin)} \mathrm{for} 6$ hours at $21 \% \mathrm{O}_{2}$. Activated NK cells were co-cultured with target cells at 1:1 ratio in 96 well round bottom plates in duplicate in the presence of anti-CD107a-Horizon-V450 (H4A3, BD). After 1 hour, co-culture at 21\% $\mathrm{O}_{2}$ or $0.6 \% \mathrm{O}_{2}$, monensin was added. After another 8-9 hours, co-cultures were stained on ice with anti-CD3-APC/H7 (SK7, BD), anti-CD56-PeCY7 (B159, BD), anti-KIR2DL1-APC (143211, R\&D), anti-2DL2/3/S2-PE (DX27, Miltenyi Biotec), anti-3DL1-FITC (DX9, Miltenyi Biotec), and anti-NKG2A-PC5.5 (Z199, Beckman Coulter). For co-cultures with IL-2 activated NK cells, target cells were pre-incubated for 6 hours at $21 \% \mathrm{O}_{2}$ or $0.6 \% \mathrm{O}_{2}$. For experiments without IL-2 activation, target cells cultured at $21 \% \mathrm{O}_{2}$ and NK cells immediately after isolation were co-cultured in a 10-12 hour degranulation assay. For HLA-E blocking experiments, Target cells were pre-incubated for 30 minutes at $37^{\circ} \mathrm{C}$ with $10 \mu \mathrm{g} / \mathrm{ml}$ of anti-HLA-E (3D12; eBioscience) or IgG1 isotype-control before addition of unactivated NK cells. Gating strategy for analysis of CD107a in NK cell subsets is described in Figure S3.2.

\section{HLA-E induction by peptides}

U266 cells were incubated with 500 $\mu \mathrm{M}$ of HLA-A1- (VMAPRTLLL), HLA-B7- (VMAPRTVLL) or a control-peptide (RGPGRAFVTI) for 12 hours at $37^{\circ} \mathrm{C}$. As additional negative controls, U266 cells were incubated without peptide or with DMSO, the solvent of the peptides. After 12 hours, HLA-E expression was determined by flowcytometry. For 
degranulation assays peptide-incubation was done for 2 hours after which the cells were co-cultured with IL-2 activated NK cells in a 12-hour degranulation assay.

\section{Mice and Multiple Myeloma disseminated growth model in $\mathrm{RAG}^{-/} \mathrm{\gamma c}^{-/-}$ mice}

RAG $-2^{-1} \mathrm{Yc}^{-/-}$mice were bred in the SPF unit Central Animal Facility of the Utrecht University using filter top cages. After a total body irradiation (3.0G $200 \mathrm{kV} \mathrm{X-rays)} \mathrm{the}$ mice received an injection with $5 \times 10^{6}$ cells intravenously from the GFP-luciferase marked, human MM cell line U266-luc. Tumor growth of the U266-luc cell line was monitored by bioluminescence imaging as described previously ${ }^{37}$. Upon sacrifice of the mice, BM cells were stained for human-CD45, HLA-class I and HLA-E.

\section{Statistical analyses}

Statistical significances of differences were determined by paired $t$-test or Two way Repeated Measures ANOVA with Bonferroni correction. A $P$-value of $<0.05$ was considered significant. Analysis was done with GraphPad Prism V (Graphpad Software Inc).

\section{Results}

To study HLA expression on primary myeloma cells, cells were obtained from BM aspirates of eight myeloma patients and one plasma cell leukemia ( $P C L$ ) patient. Directly upon isolation, surface expression of HLA-class I and HLA-E was analyzed by flowcytometry. Plasma cells were identified as CD38 ${ }^{\text {high }}$ and displayed skewed intracellular expression of either kappa- or a lambda-light chain indicative for myeloma (Figure S3.1). In all myeloma patients, $\mathrm{CD} 38^{\text {high }}$ cells were positive for HLA-E and HLAclass I (Figure 3.1). Also, CD38 ${ }^{\text {high }}$ cells from the PCL patient expressed HLA-E. The level of HLA-E and HLA-class I on CD38 ${ }^{\text {high }}$ cells was comparable to the level observed on normal BM cells of the same patient or on plasma cells from a non-myeloma patient (data not shown). 
A
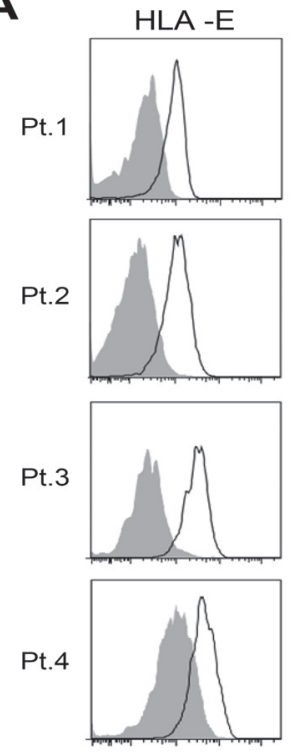

PCL

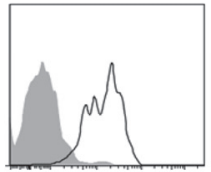

HLA class I
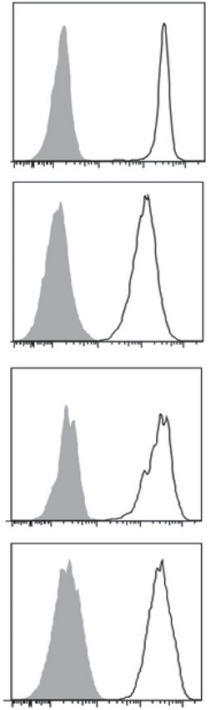

not analyzed
HLA -E

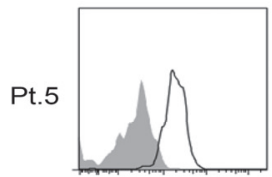

Pt.6

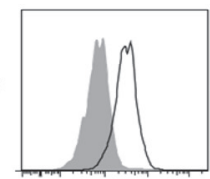

Pt.7

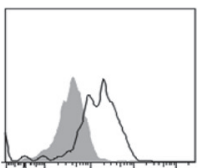

Pt.8

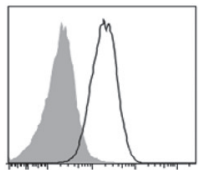

HLA class I
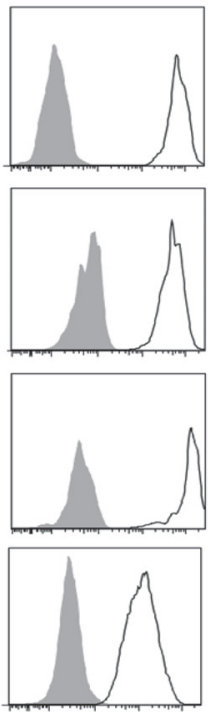

B
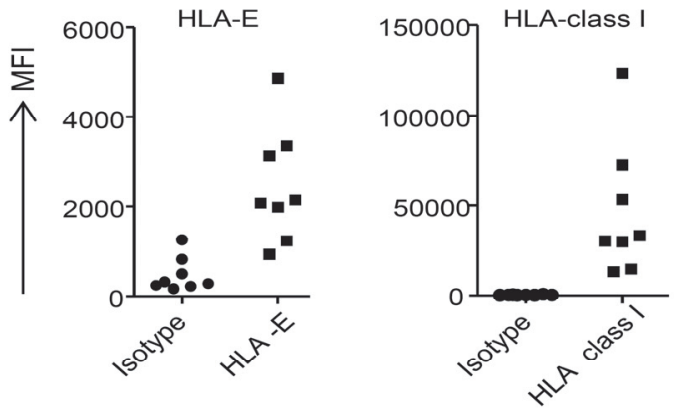

Figure 3.1 Patient derived primary myeloma cells express HLA-class I and HLA-E on the cell surface.

Cells obtained from bone marrow aspirates from patients with myeloma $(n=8)$ or plasma cell leukemia ( $P C L ; n=1$ ) were stained for CD38, HLA-class I and HLA-E and were analyzed by flowcytometry. (A) Histograms show HLA-class I or HLA-E cell surface expression (open histograms) on $\mathrm{CD} 38^{\text {high }}$ cells for the myeloma or PCL patients. Matched isotype controls are depicted in grey histograms. (B) Graph shows MFI data obtained from the histograms. Each dot depicts data from one patient. 


\section{Myeloma cell lines express high levels of HLA-class I and heterogeneous levels of HLA-E.}

Surface expression of HLA-class I and HLA-E was also assessed on a panel of myeloma cell lines including U266, L-363, LME-1, UM-9, RPMI-8226/S, OPM-1 and XG-1, and on the leukemia cell line K562. This revealed that all myeloma cell lines strongly expressed HLA-class I (Figure 3.2A). K562 cells were almost completely negative for HLA-class I. The cell lines differed in expression levels of HLA-E; K562 and OPM-1 lacked cell surface HLA-E while U266, L-363, UM-9, LME-1 and RPMI-8226/S expressed low levels of HLA-E (less than 1 log difference with the isotype-control). XG-1 expressed intermediate HLAE levels (approximately 1 log difference with the isotype-control) (Figure 3.2B).

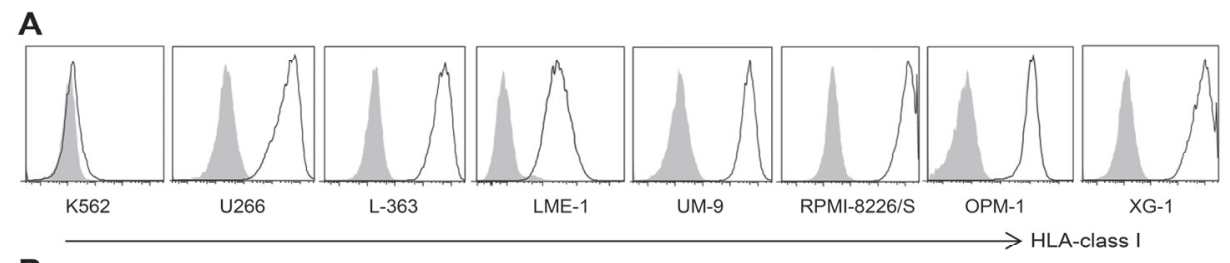

B

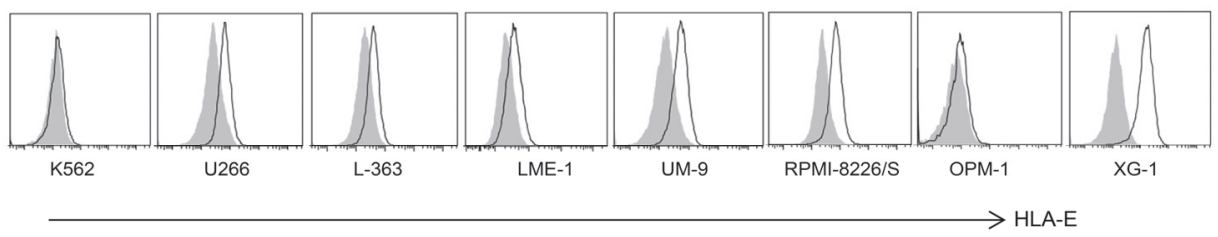

Isotype control

Anti-HLA

Figure 3.2 Myeloma cell lines express high levels of HLA-class I and heterogeneous levels of HLA-E.

HLA-class I (A) and HLA-E (B) surface expression of HLA-class I deficient K562, and six myeloma cell lines (U266, L-363, LME-1, UM-9, RPMI-8226/S, OPM-1, XG-1) was determined by flowcytometry. Histograms are representative of three different measurements. HLA expression is depicted by open histograms and matched isotype controls by grey histograms.

\section{In vivo grown U266 myeloma cells express higher levels of HLA-E than in vitro grown U266 cells}

As we observed a clear expression of HLA-E by all patient-derived CD38 ${ }^{\text {high }}$ cells but only low expression on in vitro cultured myeloma cell lines, we compared HLA-E expression on in vitro grown U266 cells with U266 cells after in vivo passaging. To this end, GFPluciferase marked U266 cells were injected in RAG- $2^{-/-} \mathrm{yc}^{-1}$ mice thereby providing the cells with their natural BM environment. Tumor growth was monitored with bioluminescence imaging. At end-stage myeloma development, the BM was harvested and tumor cells, identified by GFP and human leucocyte marker CD45, were analyzed for surface HLA-E and HLA-class I. This analysis revealed that both in vitro and in vivo 
grown U266 cells strongly expressed HLA-class I, albeit that the in vivo level was somewhat lower than the in vitro level. A striking observation was that the in vivo passaged U266 cells expressed much higher levels of HLA-E when compared to U266 cells grown in vitro; MFI HLA-E divided by MFI isoype-control was 30.6 in vivo vs. 2.4 in vitro (Figure 3.3).

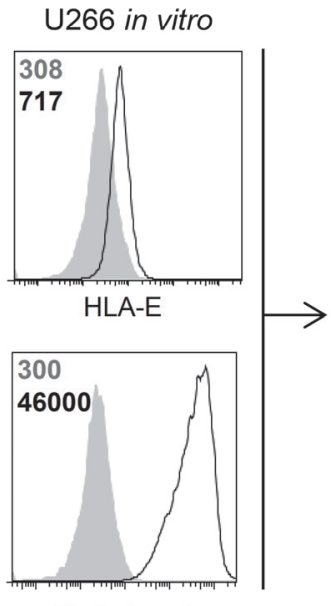

HLA class I

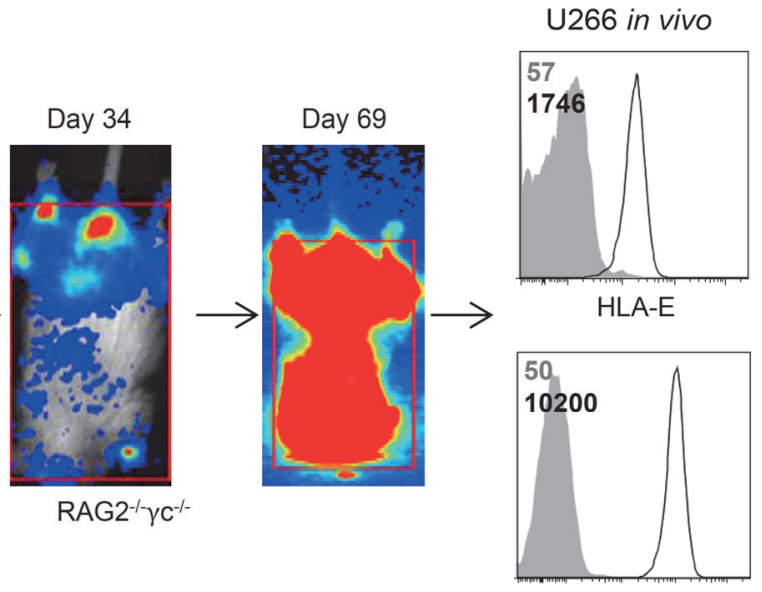

HLA class I
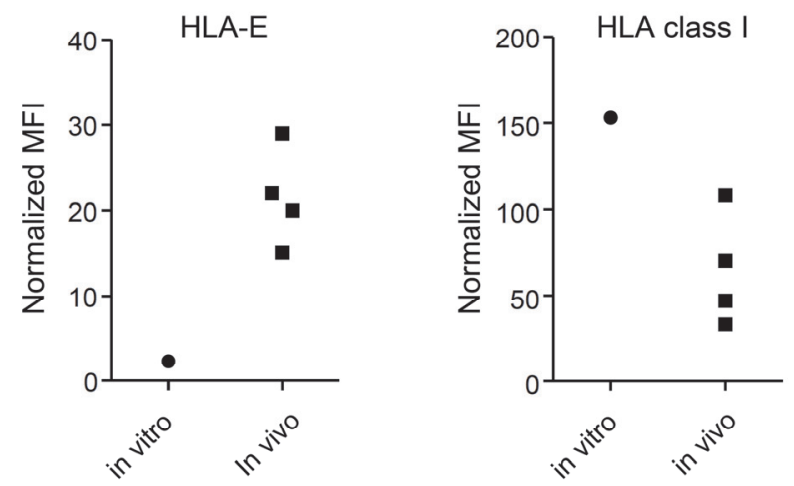

Figure 3.3 In vivo grown U266 myeloma cells have a higher HLA-E expression than in vitro cultured U266 cells.

$5 \times 10^{6}$ U266 cells were injected in RAG- $2^{-/} \gamma \mathrm{c}^{-/-}$immunodeficient mice. Tumor growth was monitored at multiple timepoints after injection by bioluminescent imaging. After reaching the human endpoint, mice were sacrificed and the bone marrow was harvested and stained for HLA-class I and HLA-E. U266 cells grown in vitro were also stained for HLA-class I and HLA-E. HLA expression was analyzed by flowcytometry. Representative histograms are shown of HLA expression (open histograms) and matched isotype controls (grey histograms). Numbers in the histograms depict MFI of the isotype control (grey) or HLA (black). Graph shows normalized MFI's obtained from these histograms (MFI of HLA divided by MFI of isotype control). Each dot represents one mouse. 


\section{KIR-ligand mismatched NK cell subsets mediate the most effective anti- myeloma response}

To evaluate the functional relevance of HLA for NK cell anti-myeloma alloreactivity, myeloma cell lines were co-cultured with NK cells from donors expressing all three inhibitory epitopes (i.e. HLA-C1, -C2 and -Bw4+). To enable comparative analysis of antimyeloma activity of NK cell subsets, cells were stained for KIRs and NKG2A and NK cell degranulation of subsets was assessed by flowcytometric analysis of the degranulation marker CD107a (Figure S3.2). Previously, others and we showed that CD107a is a reliable surrogate marker for NK cell cytotoxicity ${ }^{24,38}$ and traditional cytotoxicity assays as such would not allow the analysis of subgroups without cell sorting.

To assess the importance of KIR-HLA class I-KIR interaction, NKG2A-KIR+ NK cells were classified into three subsets based on the target HLA-class I genotype (Table S3.1); a subset exclusively expressing matched KIRs, a subset expressing only mismatched KIRs, and a subset co-expressing matched and mismatched KIRs. For all three subsets, the percentage of CD107a+ cells was below $1 \%$ without target cells (Figure 3.4A). The percentage of degranulating CD107a+ matched NK cells upon culture with myeloma cell lines was also low; $5.1 \%$ with OPM-1 and $<2.2 \%$ with the other cell lines. For the mismatched subsets, the percentage of degranulating NK cells was higher than the percentage of the matched subset. The magnitude differed between the target cell lines; $20.1 \%$ with U266; $12.7 \%$ with L-363; $10.3 \%$ with LME-1; $2.9 \%$ with UM-9; $2.1 \%$ with RPMI-8226/S; and $15.8 \%$ with OPM-1. For the NK cell subset co-expressing both matched and mismatched KIRs percentages of CD107a+ cells were in between that of the subset expressing matched KIR and the subset exclusively expressing mismatched KIR. Together these data illustrated that anti-myeloma NK cell reactivity is abrogated by HLA-class I.

Since clinical NK cell products usually consist of cytokine-activated or -expanded NK cells ${ }^{16,39,40}$, we investigated whether our observation that unactivated KIR-ligand mismatched NK cells are better responders to myeloma than matched NK cells was also true for activated NK cells. To test this, NK cells were activated for 6 hours with IL-2 prior to the analysis of degranulation of the different NK cell subsets. Spontaneous degranulation of activated NK cells was again low $(<0.5 \%)$. For matched NK cells the percentage of CD107a+ cells in response to myeloma target cells was $10.6 \%$ with U266, $7.8 \%$ with L-363, 6.4\% with RPMI-8226/S and 24\% with OPM-1 (Figure 3.4B). In line with our first observation, NK cells that were KIR-ligand mismatched showed a significantly better anti-MM response than the matched subsets; the percentages of CD107a+ cells were; 54\% with U266; 54.8\% with L-363; 32.6\% with RPMI-8226/S and $43.2 \%$ with OPM-1. 

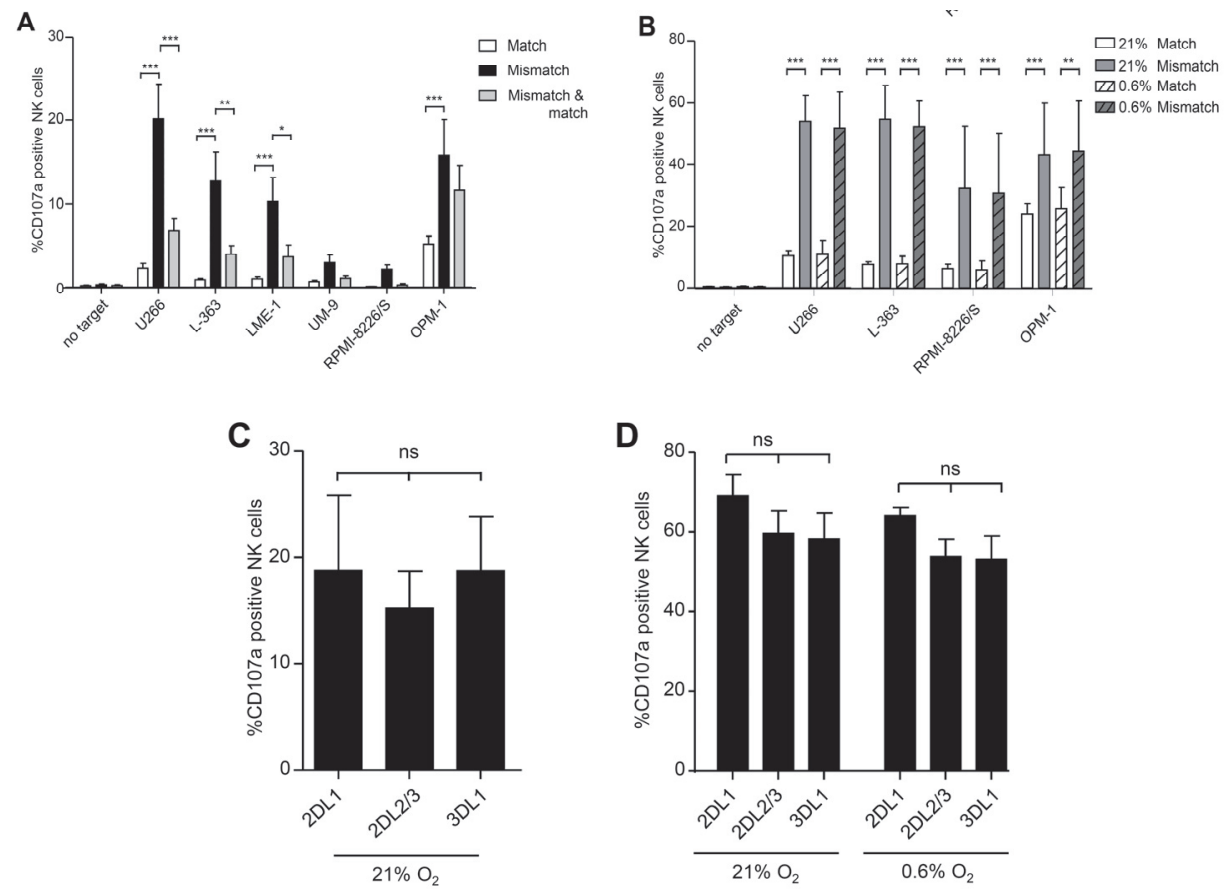

Figure 3.4 KIR-ligand mismatched NK cell subsets mediate the most effective anti-myeloma response.

(A) Six myeloma cell lines were co-cultured with NK cells isolated from peripheral blood in the presence of anti-CD107a at $21 \%$ of oxygen. After 12 hours, cells were stained for KIRs and NKG2A, and degranulation (CD107a+) was measured by flow cytometry. For analysis, NK cells (CD3-CD56+) were subdivided into 16 subpopulations based on their expression of different inhibitory receptors and the percentage of CD107a+ cells per subset was analyzed. Next, the 16 populations were categorized into 3 sub-groups, KIR-ligand matched NK cells (white bars), KIRligand mismatched NK cells (black bars) and NK cells with a co-expression of KIR-ligand mismatched and matched cells (grey bars). This was done per myeloma cell line based on the HLA genotype of the cell line. For all subsets, NKG2A+ NK cells were excluded from the analysis. Graph shows mean percentage and SEM of CD107a+ NK cells for each of these subgroups. (B) Myeloma cell lines were pre-incubated for 6 hours at $21 \%$ or $0.6 \% \mathrm{O}_{2}$. NK cells were activated for 6 hours with $1000 \mathrm{IU} / \mathrm{ml} \mathrm{IL-2}$ at $21 \%$ of oxygen. After 6 hours, NK cells and myeloma cells were co-cultured for an additional 10 hours at $21 \%$ or $0.6 \% \mathrm{O}_{2}$. Analysis of degranulation of IL-2 activated NK cells subsets was performed as described under A. Graph shows mean percentage and SEM of CD107a+ NK cells for NK cells exclusively expressing matched (white bars) or mismatched $\mathrm{KIRS}$ (grey bars) at $21 \% \mathrm{O}_{2}$ (open bars) or $0.6 \% \mathrm{O}_{2}$ (dashed bars). (C-D) Unactivated NK cells (C) or activated NK cells (D) were cocultured with K562 as described in 4A and B. Graphs in C and D show mean percentage and SEM of CD107a+ NK cells for subsets exclusively expressing KIR2DL1 or KIR2DL2/3 or KIR3DL1. Experiments in A and $\mathrm{C}$ were performed in the same assay in duplicate cultures for $n=4$ healthy donors. Experiments in $B$ and $D$ were performed in the same assay in duplicate cultures for $\mathrm{n}=5$ donors. ${ }^{*} P<0.05, * * P<0.01$, $* * * P<0.001$

Previously, we showed that hypoxia can inhibit the cytotoxic capacity of NK cells and that IL-2 NK cell activation can compensate for that ${ }^{24}$. As the myeloma microenvironment exhibits hypoxic regions ${ }^{41}$, we also determined degranulation of IL-2 
activated NK cell subsets at physiologically relevant hypoxic culture conditions of $0.6 \%$ $\mathrm{O}_{2}$. In response to all tested cell lines and for both matched and mismatched subsets the percentage of CD107a+ cells was comparable to the levels observed at $21 \%$ of oxygen. Thus, also under hypoxic culture conditions, KIR-ligand mismatched subsets displayed superior anti-myeloma activity (Figure 3.4B).

As a control, degranulation of KIR2DL1, KIR2DL2/3 or KIR3DL1 single positive NK cells in response to the HLA-class I negative $\mathrm{K} 562$ cell line was determined. For the unactivated NK cells this showed that all three subsets had around 18-20\% CD107a+ NK cells upon incubation with $\mathrm{K} 562$ (Figure 3.4C). Also for the activated NK cells, there was no significant difference in the percentage of CD107a+ cells between KIR2DL1, KIR2DL2/3 or KIR3DL1 single positive subsets, neither at $21 \% \mathrm{O}_{2}$ nor at $0.6 \% \mathrm{O}_{2}$ (Figure 3.4D) demonstrating that all subsets can degranulate to a comparable extent in the absence of inhibitory signaling by KIR-ligand interaction.

\section{HLA-E can abrogate the anti-myeloma response of NKG2A+ NK cells}

To evaluate the functional relevance of HLA-E, we used the experimental set up above, and determined degranulation of NK cells expressing only the inhibitory receptor for HLA-E; i.e. NKG2A. In the absence of target cells, $1 \%$ of KIR-NKG2A+ NK cells was positive for CD107a (Figure 3.5A). Between 16 and 21\% of KIR-NKG2A+ NK cells expressed CD107a upon co-culture with U266, L-363 and OPM-1. This percentage was only slightly lower than the percentage observed with the HLA-negative K562 suggesting that the low levels of HLA-E on U266, L-363 and OPM-1 were not sufficient to inhibit the KIR-NKG2A+ NK cells. In response to LME-1, UM-9, RPMI-8226/S, and XG-1, only $2-6 \%$ of the KIR-NKG2A+ NK cells was positive for CD107a. To investigate whether the poor response against these targets was the consequence of HLA-E, despite its low expression (Figure 3.2), HLA-E was blocked on these four cell lines with an anti-HLA-E antibody. For UM-9 or XG-1 blocking did not significantly increase NK cell degranulation as compared to the isotype-control (Figure 3.5B). However, for LME-1 or RPMI8226/S blocking enhanced degranulation of KIR-NKG2A+ NK subsets of all four donors. HLA-E blocking did not enhance degranulation of KIR+NKG2A-subsets (data not shown). Hence, although HLA-E is not the only contributing factor, our data show that HLA-E can clearly inhibit the anti-myeloma response of KIR-NKG2A+ NK cells.

We also studied degranulation of IL-2 activated KIR-NKG2A+ NK cells upon co-culture with K562, U266, L-363, RPMI-8226/S and OPM-1. Spontaneous degranulation without target cells was $5 \%$. In response to K562, 60\% of the KIR-NKG2A+ NK cells expressed CD107a while this was $30 \%-50 \%$ in response to the myeloma cells (Figure $3.5 \mathrm{C}$ ). This phenomenon was also valid for targeting myeloma in a hypoxic environment of $0.6 \%$ oxygen showing that degranulation of activated NK cells was not severely hampered by the low levels of HLA-E present on the myeloma cell lines. 

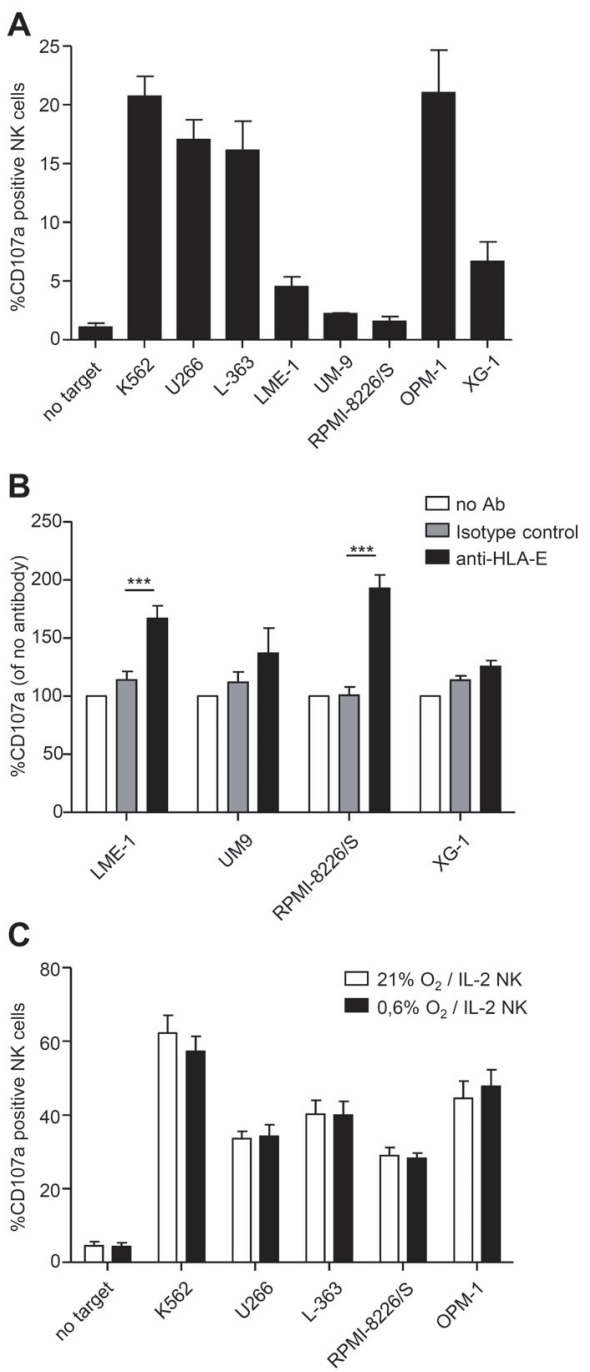

Figure 3.5 HLA-E can abrogate the anti-myeloma response of NKG2A+ NK cells.

(A) The tumor cell lines were co-cultured at $21 \% \mathrm{O}_{2}$ with freshly isolated peripheral blood derived NK cells for 12 hours, in the same assay as in figure $4 \mathrm{~A} / \mathrm{C}$. NK cells expressing only NKG2A as an inhibitory receptor were analyzed downstream for CD107a expression. Graph shows mean percentage and SEM CD107a+ of NKG2A+KIR- NK cells. (B) LME-1, UM-9, RPMI$8226 / \mathrm{S}$, and XG-1 were co-cultured with non-activated NK cells for 12 hours at $21 \% \mathrm{O}_{2}$, in presence of an HLA-E blocking antibody (black bars), an IgG1 isotype control (grey bars) or without antibody (white bars). Graph shows mean normalized percentage CD107a+ cells of NKG2A+KIR- NK cells (\% with Ab divided by \% without antibody). (C) Myeloma cell lines were pre-incubated for 6 hours at $21 \%$ or $0.6 \% \mathrm{O}_{2}$. NK cells were activated with $1000 \mathrm{IU}$ IL-2 at $21 \%$ $\mathrm{O}_{2}$. Thereafter, they were co-cultured with K562, U266, L363, RPMI-8226/S, and OPM-1, for 10 hours, at $21 \% \mathrm{O}_{2}$ or at $0.6 \% \mathrm{O}_{2}$, and CD107a expression on KIR-NKG2A+ NK cells was analyzed as described in Figure 3.5. Graph shows mean percentage and SEM CD107a+ of NKG2A+KIR- NK cells. All experiments were performed in duplicate and with $\mathrm{n}=5$ donors. ${ }^{* * *} P<0.001$ 


\section{Upregulation of HLA-E on myeloma cells diminishes degranulation of IL-2 activated NKG2A+ NK cells}

Most of the cell lines in our panel expressed relatively low levels of HLA-E as compared to the levels observed in vivo in myeloma patients and in mice, and therefore we questioned whether a higher, more clinically relevant level of HLA-E expression would inhibit degranulation of activated NKG2A+ NK cells. HLA-E expression was enhanced on one of the HLA-E ${ }^{\text {low }}$ cell lines (U266) by pre-incubation with A1- or B7-peptides. Both peptides are natural binders of HLA-E derived from the leader sequence of HLA-A1 or HLA-B7 allotypes and have been shown to enhance surface HLA-E levels by stabilization of the molecule on the cell surface ${ }^{12}$ Indeed, the incubation with either A1- or B7-peptide enhanced the level of HLA-E on U266 as compared to a non-HLA-E binding control peptide or DMSO (Figure 3.6A). Subsequent co-culture experiments with U266 and IL-2 activated allogeneic NK cells revealed that in the control conditions (U266 alone, with DMSO or control peptide) $40-50 \%$ of the NK cells expressed CD107a (Figure 3.6B). In response to U266 pre-incubated with A1- or B7-peptide, the percentage CD107a+ KIR-NKG2A+ NK cells was reduced from $40-50 \%$ to only $15-20 \%$. Degranulation of KIR+NKG2A- NK cells was not influenced by pre-incubation of U266 cells with A1- or B7-peptide. These data support that the up-regulation of HLA-E on myeloma cells that are in principle sensitive to NK cell kill become resistant to NKG2A+ NK cells, indicating that the selection of NKG2A negative NK cells is essential to efficiently eliminate HLA-E positive myeloma cells.

\section{Discussion}

Despite the introduction of novel drugs to treat Multiple Myeloma this disease has remained largely incurable. NK cells can be novel immunotherapeutic candidates, and their safety has been documented in clinical trials ${ }^{17}$. Exploitation of the full potential of NK cells requires selection of NK cell subsets with the highest possibility of triggering anti-myeloma, and identification of the factors important for sustaining NK cell activity in vivo. In quest of further refining NK cell therapy, we evaluated the clinical relevance of HLA-class I and HLA-E for the anti-myeloma response of alloreactive NK cells.

Expression of HLA-E in primary myeloma has not been reported and the present study shows that HLA-E is indeed expressed by CD38 ${ }^{\text {high }}$ cells of myeloma or PCL patients. Our data also demonstrate that primary myeloma cells remain positive for HLA-class I as has been described before ${ }^{28-30}$. HLA-class I was also strongly expressed by all cell lines in our panel. HLA-E expression, on the other hand, was lower on cell lines than on primary myeloma cells, and ranged from completely absent e.g. on OPM-1 to very low on most of the other myeloma lines. Low levels of HLA-E on solid tumor cell lines were also reported in three previous studies with 30-37 different in vitro cultured cell lines per study $^{42-44}$. The most striking observation in our study was that the HLA-E level on U266 
cells grown in the BM of RAG-2/- $\mathrm{Yc}^{-/-}$mice was considerably higher than the level on in vitro cultured U266, and relatively comparable to the level in patients. Thus, there seems to be a difference between in vitro and in vivo expression levels of HLA, which might be the consequence of supportive factors present in vivo that lack in vitro. This observation is highly relevant for the interpretation of in vitro NK cell studies using HLA$\mathrm{E}^{\text {low }}$ tumor cells in general; because the in vitro situation might be very limiting in predicting the actual situation in cancer patients.

In a co-culture system allowing the synchronized analysis of degranulation of NK cell subsets, we identified HLA-E as a potent inhibitory factor for anti-myeloma reactivity of NK cells expressing the inhibitory NKG2A receptor (Figure 3.5). The impact of HLA-E was most pronounced in the experiment where blocking of HLA-E enhanced degranulation of KIR-NKG2A+ NK cells, and in the experiment where HLA-E binding peptides reduced KIR-NKG2A+ NK cell degranulation via the upregulation of HLA-E which is consistent with the inhibitory role of HLA-E described in $A M L$ and $A L L^{32}$. Because a large fraction of circulating NK cells and the majority of NK cells in clinical products is NKG2A+, HLA-E can be expected to have major impact on overall NK cell alloreactivity against HLA-E expressing tumors. HLA-E mediated tumor resistance might at least partly explain the negative clinical results obtained so far. Our current data provide a strong argument to use NKG2A negative NK cells in future clinical trials or develop strategies to abrogate inhibition by HLA-E.

Our findings consequently demonstrated a higher killing potential of KIR-ligand mismatched NK cells as compared to their matched counterparts. Previously, bortezomib-induced downregulation of HLA-class I ${ }^{45}$, KIR-blockade with IPH $2011^{46}$ and HLA-blockade with W6/32 ${ }^{16}$ have been shown to enhance killing of myeloma cells by NK cells. Our data are in agreement with these studies and confirm the relevance of HLAclass $I$ in an alternative experimental set up. An advantage of our system is that it allows the simultaneous analysis of individual subsets, which additionally revealed that the anti-myeloma advantage of mismatched NK cells was reduced by co-expression of one or more matched KIR. Hence, selection of NK cell subsets with exclusively ligand mismatched KIRs will be important to enhance clinical efficacy.

We recently showed that IL-2 activation is required for NK cell anti-myeloma responses in clinically relevant hypoxic conditions ${ }^{24}$. Here, we demonstrate that, even though IL-2 activation enhanced degranulation of all NK cell subsets, KIR-ligand mismatched NK cells still mediated a more potent anti-myeloma response than matched NK cells. Importantly, we did not observe a difference between anti-myeloma responses at $21 \%$ and $0.6 \% \mathrm{O}_{2}$ indicating that for all subsets activation was sufficient to overcome the inhibitory effect of hypoxia. Recently, pre-activation of NK cells with a combination of IL-12/-15/-18 proved to be more efficient in enhancing NK cell effector function as compared to activation with IL-2 or IL-15 alone ${ }^{47,48}$. Thus, cytokines other than IL-2 might enhance the therapeutic potential of alloreactive NK cells even further. 
A

- Isotype stair

- Medium con

- DMSO contr

- Control pept

- B7 peptide

- A1 peptide
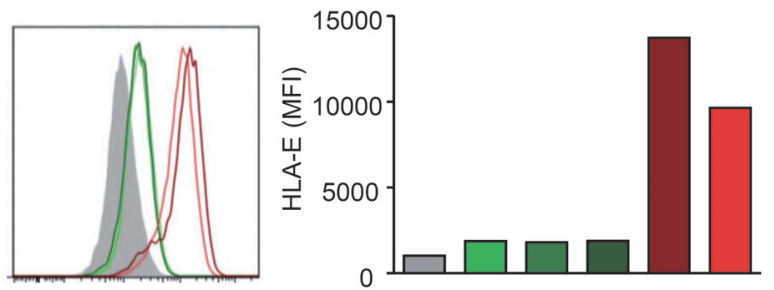

anti-HLA-E

B
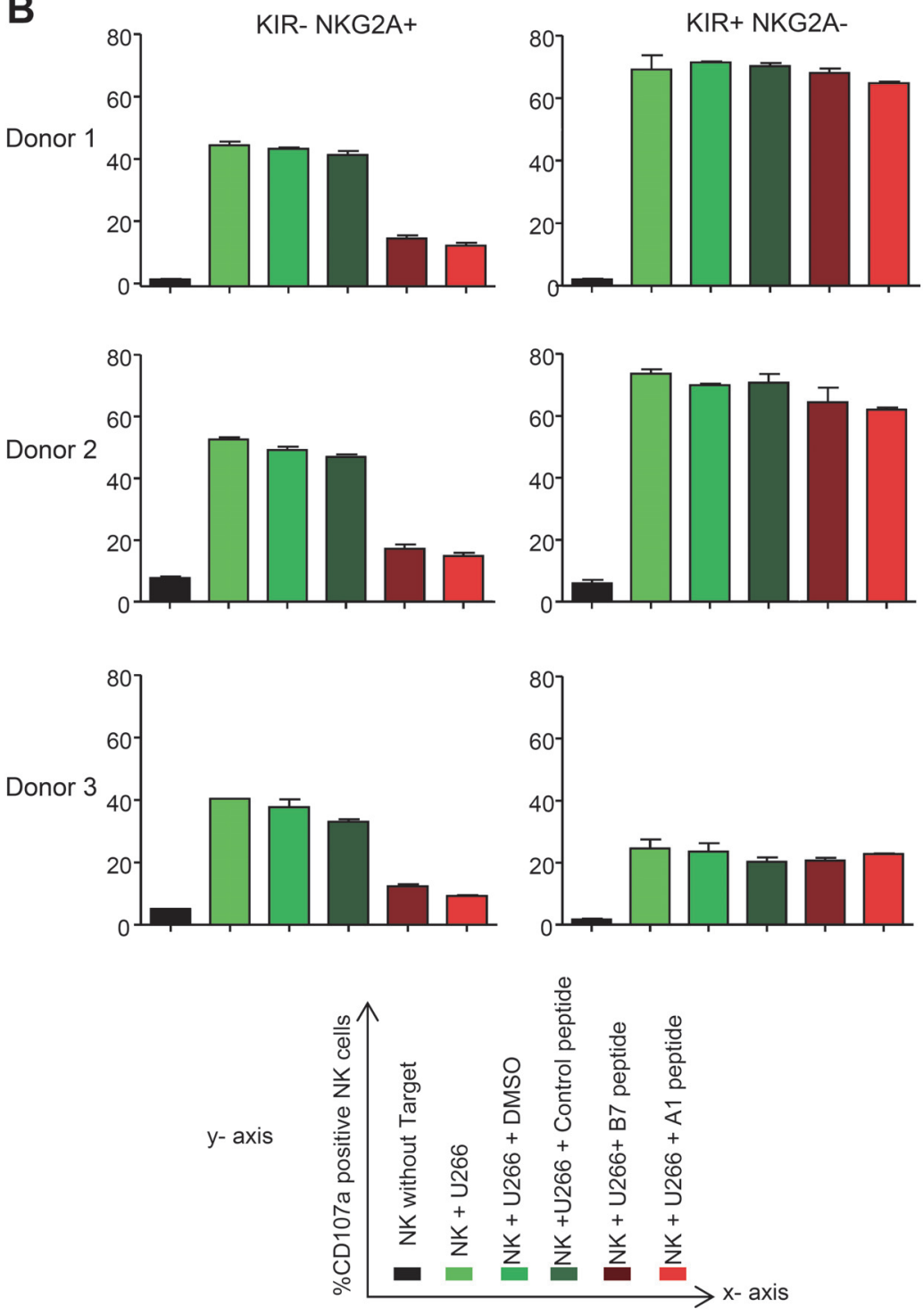
Figure 3.6 Upregulation of HLA-E renders NK-sensitive myeloma cells resistant to IL-2 activated NKG2A+ NK cells.

(A) U266 cells were incubated for 12 hours at $37^{\circ} \mathrm{C}$ with $500 \mu \mathrm{M}$ A1- or B7- HLA-E binding peptides. As control U266 cells were incubated with a control (non-binding) peptide, DMSO or medium. HLA-E surface expression was determined by flowcytometry after staining of the cells with anti-HLA-E or an IgG1 isotype control. Shown is a representative histogram with in grey the isoype control staining, in green the control incubations and in red the conditions with HLA-E binding peptides. Graph shows MFI values from the histogram. (B) Myeloma cells were preincubated with peptides and controls as described in A for two hours. Then, IL-2 activated NK cells were added and the cells were co-cultured for an additional 12 hours. Percentage of CD107a+ cells was determined for three donors in NKG2A+KIR- and in NKG2A-KIR+ NK cell subsets. Graphs depict means of duplicate cultures per donor. X-axes legend is given in the lower panel of the figure.

We acknowledge that in our system HLA-class I and HLA-E were not the only factors determining the magnitude of the anti-myeloma response; i.e. HLA-E blocking did not completely restore degranulation, and in response to some cell lines (e.g. UM-9) even fully KIR-ligand mismatched NK cells did not vigorously degranulate (Figure 3.4A). Previously, the activating receptors NKG2D, NCRs and DNAM-1 have shown to be important for NK cell anti-myeloma activity ${ }^{28,29,49}$. Although our study was not designed to investigate the role of activating ligands, analysis of the NKG2D ligands MICA/B and ULPB2 showed that most myeloma cell lines in our panel expressed at least one of these ligands (Table S3.2). UM-9, however, expressed very low levels of all three ligands, which might explain the low capacity to activate NK cells. To develop effective immunotherapeutic strategies for resistant myeloma, synergy between alloreactive NK cells and drugs like lenalidomide and 17-AAG should be evaluated because these drugs have been shown to sensitize myeloma by enhancing the expression of activating ligands ${ }^{46,50}$.

In summary, our results show that KIR-HLA-class I and NKG2A-HLA-E interactions are highly relevant for NK cell reactivity against myeloma. For accurate prediction of in vitro data to patient's reality, two relevant in vivo realities have to be taken into account: 1) in the bone marrow myeloma cells reside under hypoxic conditions and 2) in vivo, myeloma cells express both HLA-class I and HLA-E. Infusion of a high number of cytokine activated alloreactive NKG2A negative, KIR-ligand mismatched NK cells may help to improve the efficacy of alloreactive NK cell therapy.

\section{Acknowledgements}

The RAG ${ }^{-1} \mathrm{YC}^{-1-}$ mice were originally obtained from the Amsterdam Medical Center (Amsterdam, The Netherlands). The authors would like to thank N. Lauterbach for assistance with the HLA-E peptide experiments, the diagnostic hematology laboratory (MUMC+) for HLA staining of the patient bone marrow samples and the tissue typing laboratory (MUMC+) for HLA typing of the cell lines. S. Sarkar was supported by a PhD grant from GROW, school of oncology and developmental biology. L. Wieten was 
supported by a personal grant from Dutch Cancer association (KWF kankerbestrijding; UM2012-5375). G. Bos, M. van Gelder and L. Wieten were supported by a grant from the Kankeronderzoeksfonds Limburg. 


\section{REFERENCES}

1. Palumbo A, Anderson K (2011) Multiple myeloma. N Engl J Med 364: 1046-1060.

2. Kroger N, Shaw B, lacobelli S, Zabelina T, Peggs K, et al. (2005) Comparison between antithymocyte globulin and alemtuzumab and the possible impact of KIR-ligand mismatch after dose-reduced conditioning and unrelated stem cell transplantation in patients with multiple myeloma. $\mathrm{Br} \mathrm{J}$ Haematol 129: 631-643.

3. Shimoni A, Hardan I, Ayuk F, Schilling G, Atanackovic D, et al. (2010)Allogenic hematopoietic stem-cell transplantation with reduced-intensity conditioning in patients with refractory and recurrent multiple myeloma: long-term follow-up. Cancer 116: 3621-3630.

4. El-Cheikh J, Crocchiolo R, Furst S, Stoppa AM, Ladaique P, et al. (2013) Long-term outcome after allogeneic stem-cell transplantation with reduced-intensity conditioning in patients with multiple myeloma. Am J Hematol 88: 370-374.

5. Koehne G, Giralt S (2012) Allogeneic hematopoietic stem cell transplantation for multiple myeloma: curative but not the standard of care. Curr Opin Oncol 24: 720-726.

6. Moreau P (2012) Death of frontline allo-SCT in myeloma. Blood 119: 6178-6179.

7. Palmer JM, Rajasekaran K, Thakar MS, Malarkannan S (2013) Clinical relevance of natural killer cells following hematopoietic stem cell transplantation. J Cancer 4: 25-35.

8. Vivier E, Ugolini S (2011) Natural killer cells: from basic research to treatments. Front Immunol 2: 18.

9. Vivier E, Ugolini S, Blaise D, Chabannon C, Brossay L (2012) Targeting natural killer cells and natural killer T cells in cancer. Nat Rev Immunol 12: 239-252.

10. Colonna M, Borsellino G, Falco M, Ferrara GB, Strominger JL (1993) HLA-C is the inhibitory ligand that determines dominant resistance to lysis by NK1- and NK2-specific natural killer cells. Proc Natl Acad Sci U S A 90: 12000-12004.

11. Parham P (2005) MHC class I molecules and KIRs in human history, health and survival. Nat Rev Immunol 5: 201-214.

12. Braud VM, Allan DS, O'Callaghan CA, Soderstrom K, D'Andrea A, et al. (1998) HLA-E binds to natural killer cell receptors CD94/NKG2A, B and C. Nature 391: 795-799.

13. Moretta L, Locatelli F, Pende D, Marcenaro E, Mingari MC, et al. (2011) Killer Ig-like receptor-mediated control of natural killer cell alloreactivity in haploidentical hematopoietic stem cell transplantation. Blood 117: 764-771.

14. Ruggeri L, Mancusi A, Capanni M, Urbani E, Carotti A, et al. (2007) Donor natural killer cell allorecognition of missing self in haploidentical hematopoietic transplantation for acute myeloid leukemia: challenging its predictive value. Blood 110: 433-440.

15. Ruggeri L, Capanni M, Urbani E, Perruccio K, Shlomchik WD, et al. (2002) Effectiveness of donor natural killer cell alloreactivity in mismatched hematopoietic transplants. Science 295: 2097-2100.

16. Shi J, Tricot G, Szmania S, Rosen N, Garg TK, et al. (2008) Infusion of haplo-identical killer immunoglobulin-like receptor ligand mismatched NK cells for relapsed myeloma in the setting of autologous stem cell transplantation. Br J Haematol 143: 641-653.

17. Sutlu T, Alici E (2009) Natural killer cell-based immunotherapy in cancer: current insights and future prospects. J Intern Med 266: 154-181.

18. Miller JS, Soignier Y, Panoskaltsis-Mortari A, McNearney SA, Yun GH, et al. (2005) Successful adoptive transfer and in vivo expansion of human haploidentical NK cells in patients with cancer. Blood 105: 3051-3057.

19. Bachanova V, Burns L, McKenna DH, Curtsinger J, Panoskaltsis-Mortari A, et al. (2010) Allogeneic natural killer cells for refractory lymphoma. Cancer Immunol Immunother 59: 1739-1744.

20. Ren XB, Yu JP, Cao S, Ren BZ, Li H, et al. (2007) Antitumor effect of large doses IL-2-activated HLA haploidentical peripheral blood stem cells on refractory metastatic solid tumor treatment. Cancer Biother Radiopharm 22: 223-234.

21. Costello RT, Boehrer A, Sanchez C, Mercier D, Baier C, et al. (2013) Differential expression of natural killer cell activating receptors in blood versus bone marrow in patients with monoclonal gammopathy. Immunology 139: 338-341. 
22. von Lilienfeld-Toal M, Frank S, Leyendecker C, Feyler S, Jarmin S, et al. (2010) Reduced immune effector cell NKG2D expression and increased levels of soluble NKG2D ligands in multiple myeloma may not be causally linked. Cancer Immunol Immunother 59: 829-839.

23. Fauriat C, Mallet F, Olive D, Costello RT (2006) Impaired activating receptor expression pattern in natural killer cells from patients with multiple myeloma. Leukemia 20: 732-733.

24. Sarkar S, Germeraad WT, Rouschop KM, Steeghs EM, van Gelder M, et al. (2013) Hypoxia induced impairment of NK cell cytotoxicity against multiple myeloma can be overcome by IL-2 activation of the NK cells. PLoS One 8: e64835.

25. Benson DM, Jr., Bakan CE, Mishra A, Hofmeister CC, Efebera Y, et al. (2010) The PD-1/PD-L1 axis modulates the natural killer cell versus multiple myeloma effect: a therapeutic target for CT-011, a novel monoclonal anti-PD-1 antibody. Blood 116: 2286-2294.

26. Frassanito MA, Silvestris F, Cafforio P, Silvestris N, Dammacco F (1997) IgG M-components in active myeloma patients induce a down-regulation of natural killer cell activity. Int J Clin Lab Res 27: 48-54.

27. Nielsen H, Nielsen HJ, Tvede N, Klarlund K, Mansa B, et al. (1991) Immune dysfunction in multiple myeloma. Reduced natural killer cell activity and increased levels of soluble interleukin-2 receptors. Apmis 99: 340-346.

28. Bernal M, Garrido P, Jimenez P, Carretero R, Almagro M, et al. (2009) Changes in activatory and inhibitory natural killer (NK) receptors may induce progression to multiple myeloma: implications for tumor evasion of T and NK cells. Hum Immunol 70: 854-857.

29. Carbone E, Neri P, Mesuraca M, Fulciniti MT, Otsuki T, et al. (2005) HLA class I, NKG2D, and natural cytotoxicity receptors regulate multiple myeloma cell recognition by natural killer cells. Blood 105: 251-258.

30. Frohn C, Hoppner M, Schlenke P, Kirchner H, Koritke P, et al. (2002) Anti-myeloma activity of natural killer lymphocytes. Br J Haematol 119: 660-664.

31. Fauriat C, Andersson S, Bjorklund AT, Carlsten M, Schaffer M, et al. (2008) Estimation of the size of the alloreactive NK cell repertoire: studies in individuals homozygous for the group A KIR haplotype. J Immunol 181: 6010-6019.

32. Godal R, Bachanova V, Gleason M, McCullar V, Yun GH, et al. (2010) Natural killer cell killing of acute myelogenous leukemia and acute lymphoblastic leukemia blasts by killer cell immunoglobulin-like receptor-negative natural killer cells after NKG2A and LIR-1 blockade. Biol Blood Marrow Transplant 16: 612-621.

33. Lehmann D, Spanholtz J, OsI M, Tordoir M, Lipnik K, et al. (2012) Ex vivo generated natural killer cells acquire typical natural killer receptors and display a cytotoxic gene expression profile similar to peripheral blood natural killer cells. Stem Cells Dev 21: 2926-2938.

34. Berg M, Lundqvist A, McCoy P, Jr., Samsel L, Fan Y, et al. (2009) Clinical-grade ex vivo-expanded human natural killer cells up-regulate activating receptors and death receptor ligands and have enhanced cytolytic activity against tumor cells. Cytotherapy 11: 341-355.

35. Knorr DA, Ni Z, Hermanson D, Hexum MK, Bendzick L, et al. (2013) Clinical-scale derivation of natural killer cells from human pluripotent stem cells for cancer therapy. Stem Cells Transl Med 2: 274-283.

36. Maier S, Grzeschik M, Weiss EH, Ulbrecht M (2000) Implications of HLA-E allele expression and different HLA-E ligand diversity for the regulation of NK cells. Hum Immunol 61: 1059-1065.

37. Rozemuller H, van der Spek E, Bogers-Boer LH, Zwart MC, Verweij V, et al. (2008) A bioluminescence imaging based in vivo model for preclinical testing of novel cellular immunotherapy strategies to improve the graft-versus-myeloma effect. Haematologica 93: 1049-1057.

38. Alter G, Malenfant JM, Altfeld M (2004) CD107a as a functional marker for the identification of natural killer cell activity. J Immunol Methods 294: 15-22.

39. Alici E, Sutlu T, Bjorkstrand B, Gilljam M, Stellan B, et al. (2008) Autologous antitumor activity by NK cells expanded from myeloma patients using GMP-compliant components. Blood 111: 3155-3162.

40. Spanholtz J, Preijers F, Tordoir M, Trilsbeek C, Paardekooper J, et al. (2011) Clinical-grade generation of active NK cells from cord blood hematopoietic progenitor cells for immunotherapy using a closedsystem culture process. PLoS One 6: e20740.

41. Martin SK, Diamond P, Gronthos S, Peet DJ, Zannettino AC (2011) The emerging role of hypoxia, HIF-1 and HIF-2 in multiple myeloma. Leukemia 25: 1533-1542. 
42. Palmisano GL, Contardi E, Morabito A, Gargaglione V, Ferrara GB, et al. (2005) HLA-E surface expression is independent of the availability of HLA class I signal sequence-derived peptides in human tumor cell lines. Hum Immunol 66: 1-12.

43. Marin R, Ruiz-Cabello F, Pedrinaci S, Mendez R, Jimenez P, et al. (2003) Analysis of HLA-E expression in human tumors. Immunogenetics 54: 767-775.

44. Lo Monaco E, Tremante E, Cerboni C, Melucci E, Sibilio L, et al. (2011) Human leukocyte antigen E contributes to protect tumor cells from lysis by natural killer cells. Neoplasia 13: 822-830.

45. Shi J, Tricot GJ, Garg TK, Malaviarachchi PA, Szmania SM, et al. (2008) Bortezomib down-regulates the cell-surface expression of HLA class I and enhances natural killer cell-mediated lysis of myeloma. Blood 111: 1309-1317.

46. Benson DM, Jr., Bakan CE, Zhang S, Collins SM, Liang J, et al. (2011) IPH2101, a novel anti-inhibitory KIR antibody, and lenalidomide combine to enhance the natural killer cell versus multiple myeloma effect. Blood 118: 6387-6391.

47. Ni J, Miller M, Stojanovic A, Garbi N, Cerwenka A (2012) Sustained effector function of IL-12/15/18preactivated NK cells against established tumors. J Exp Med 209: 2351-2365.

48. Romee R, Schneider SE, Leong JW, Chase JM, Keppel CR, et al. (2012) Cytokine activation induces human memory-like NK cells. Blood 120: 4751-4760.

49. El-Sherbiny YM, Meade JL, Holmes TD, McGonagle D, Mackie SL, et al. (2007) The requirement for DNAM-1, NKG2D, and NKp46 in the natural killer cell-mediated killing of myeloma cells. Cancer Res 67: 8444-8449.

50. Fionda C, Soriani A, Malgarini G, lannitto ML, Santoni A, et al. (2009) Heat shock protein-90 inhibitors increase $\mathrm{MHC}$ class I-related chain A and B ligand expression on multiple myeloma cells and their ability to trigger NK cell degranulation. J Immunol 183: 4385-4394. 


\section{Supporting information}

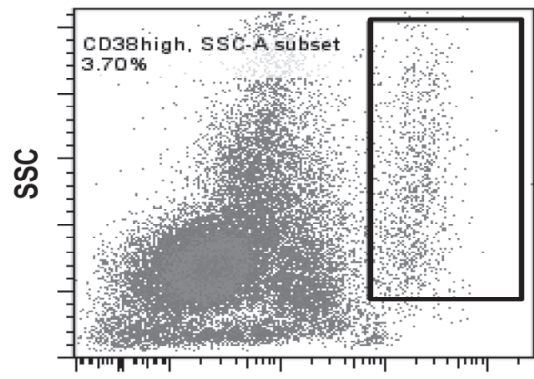

CD38

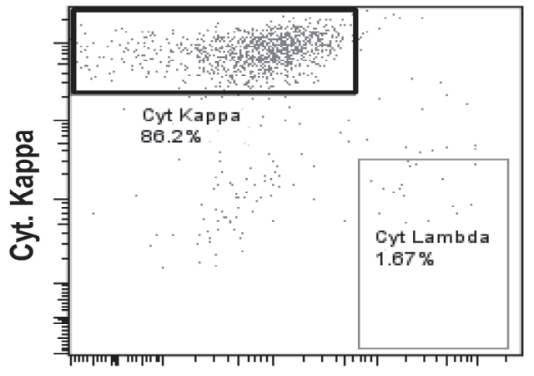

Cyt.Lambda

Figure S3.1 Patient derived primary CD38 ${ }^{\text {high }}$ myeloma cells display skewed expression of intracellular kappa- or lambda-light chain.

Cells obtained from bone marrow aspirates of patients with myeloma $(n=8)$ or plasma cell leukemia ( $P C L ; n=1$ ) were stained for surface CD38 and for intracellular kappa- and lambda-light chains and were analyzed by flowcytometry. Dotplots show representative data from one myeloma patient. Expression of intracellular kappa- and lambda-light chains is depicted for the CD38 ${ }^{\text {high }}$ subset. 

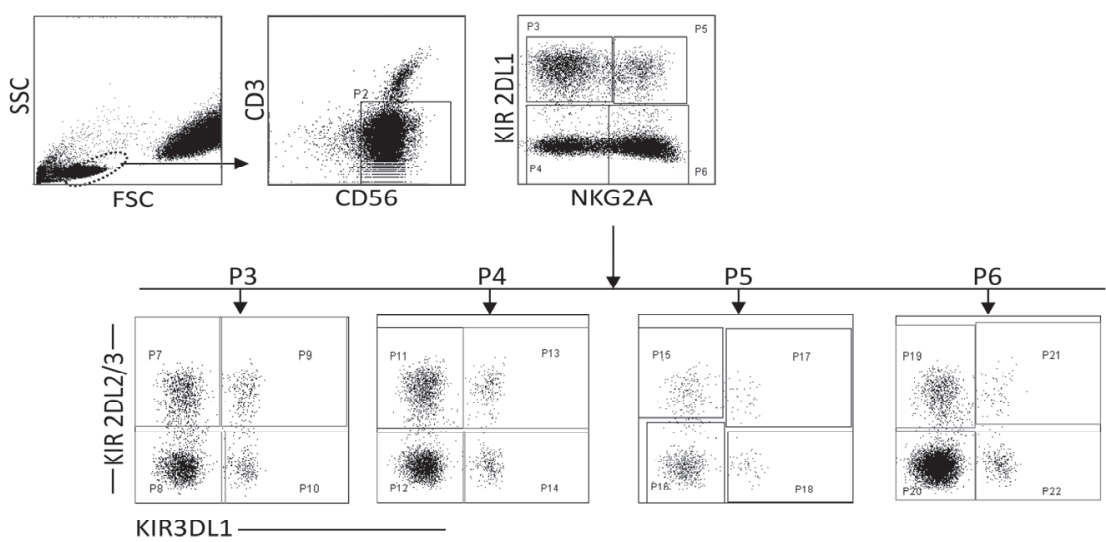

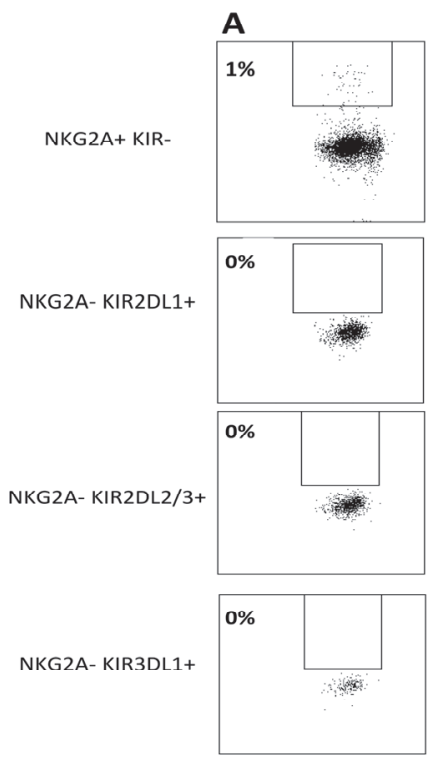

No Target
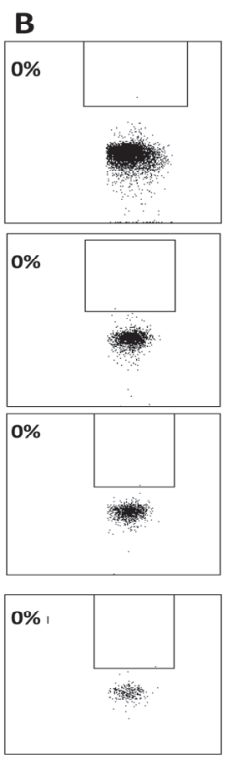

K562+Isotype

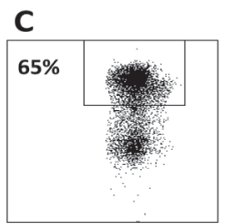

D
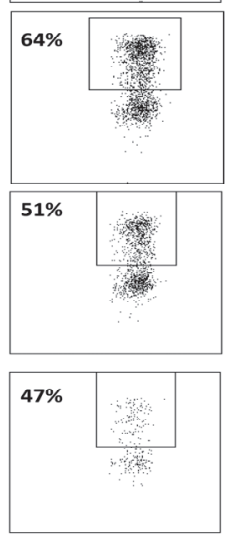

K562
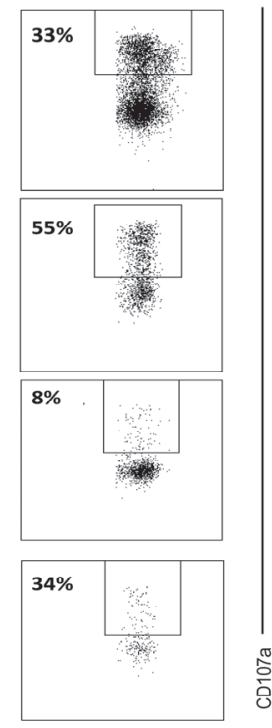

Figure S3.2 Gating strategy for CD107a degranulation assay.

Myeloma cell lines or $\mathrm{K} 562$ were co-cultured with NK cells isolated from peripheral blood in the presence of anti-CD107a (row A,C,D) or an isotype control (row B) at $21 \%$ of oxygen. After 12 hours, cells were stained for KIR and NKG2A, and degranulation (CD107a+) was measured by flow cytometry. For analysis, NK cells (CD3-CD56+) were subdivided into 16 subpopulations based on their expression of different inhibitory receptors. The percentage of CD107a+ cells in each of the 16 subsets was analyzed. Dotplots show data for the subsets expressing only one of the inhibitory receptors; i.e. single positive for NKG2A, KIR2DL1, KIR2DL2/3 or KIR3DL1 upon coculture with U266 or the positive control cell line K562. 
Table S3.1 Matched and mismatched KIRs based on genotypic expression of HLA epitopes

\begin{tabular}{llll}
\hline Cell line & HLA genotype & Matched KIR & Mismatched KIR \\
\hline U266 & C1+ C2- Bw4- & KIR2DL2/3 & KIR2DL1, KIR3DL1 \\
L363 & C1+ C2- Bw4- & KIR2DL2/3 & KIR2DL1, KIR3DL1 \\
LME-1 & C1+ C2- Bw4- & KIR2DL2/3 & KIR2DL1, KIR3DL1 \\
UM-9 & C1+ C2- Bw4- & KIR2DL2/3 & KIR2DL1, KIR3DL1 \\
RPMI-8226/s & C1+C2+ Bw4- & KIR2DL1, KIR2DL2/3 & KIR3DL1 \\
OPM-1 & C1+C2+ Bw4- & KIR2DL1, KIR2DL2/3 & KIR3DL1 \\
XG-1 & C1+C2+ Bw4+ & KIR2DL1, KIR2DL2/3, KIR3DL1 & No mismatch possible \\
\hline
\end{tabular}

Matched and mismatched KIRs for each cell line were classified based on the genotypic expression of HLAclass I epitopes as determined by luminex-SSO.

Table S3.2 Expression of activating NK cell ligands by myeloma cell lines

\begin{tabular}{lccc}
\hline Cell line & MICA & MICB & ULBP2 \\
\hline U266 & 2.8 & 3.3 & 1.1 \\
L363 & 1.5 & 1.8 & 1.4 \\
LME-1 & 0.6 & 1.9 & 1.3 \\
UM-9 & 0.7 & 1.9 & 1.2 \\
RPMI-8226/s & 2.2 & 2.2 & 1.8 \\
OPM-1 & 1.0 & 3.6 & 2.6 \\
XG-1 & 0.7 & 2.2 & 1.3 \\
K562 & 1.3 & 3.7 & 1.8 \\
\hline
\end{tabular}

Myeloma cell lines were stained for activating NKG2D ligands and expression was analyzed by flowcytometry. Table depicts relative expression (=MFI of the activating ligand divided by the MFI of the isotype of the same cell lines). Data are representative of two-three experiments. 


\section{Chapter 4}

Alloreactive Natural Killer cells have anti-

tumor capacity against disseminated human Multiple Myeloma in $\mathrm{RAG}^{-/-} \mathrm{Yc}^{-/-}$ mice when combined with low dose cyclophosphamide and total body irradiation

Subhashis Sarkar, Willy Noort, Richard Groen, Catharina H.M.J. van Elssen, Louis van Bloois, Michel van Gelder, Harry Schouten, Marcel G.J. Tilanus, Wilfred T.V. Germeraad, Lotte Wieten, Anton C.M. Martens, Gerard M.J. Bos

Submitted 


\section{Abstract}

We explored alloreactive Natural Killer (NK) cell infusion after chemo-radiotherapy conditioning as a novel immunotherapy treatment option for the incurable plasma cell malignancy Multiple Myeloma (MM). Using the RAG2 ${ }^{--} \mathrm{yc}^{-{ }^{-}}$immunodeficient disseminated MM mouse model we studied NK cell mediated Graft versus MM (GvM) responses while we concurrently examined the risk of developing graft versus host disease (GvHD). First, luciferase expressing human U266 MM cells were intravenously (i.v.) injected in RAG2 $2^{-1-} \mathrm{yc}^{-/-}$mice followed by i.v. injection with human KIR-ligand alloreactive NK cells or PBMC three weeks later. Bioluminescent imaging of tumor growth kinetics in a preliminary experiment revealed that infusion of resting NK cells decreased the MM burden in one out of four treated mice receiving the highest NK cell dose of 3 injections of $8 \times 10^{6}$ NK cells, with no signs of GvHD. Albeit that infused PBMCs efficiently eradicated established MM in all treated mice, this concurrently induced a lethal GvHD. When rhIL-2 activated NK cells were injected in subcutaneously (s.c.) growing MM tumors, they proved to be superior to resting NK cells in a dose dependent manner. However, since MM is a dispersed malignancy spread throughout the body, we next investigated the therapeutic potential of cytokine activated NK cells, from either cord blood or peripheral blood origin, in the disseminated MM mouse model. Although the activated NK cells were unable to trigger efficient GvM responses when given alone, the infusion of activated NK cells following a non-curative conditioning with cyclophosphamide and total body irradiation did prolong progression free survival ( $P=0.003)$ without any signs of GvHD. Our study illustrates the therapeutic potential and safety of NK cell therapy in MM and is an important step towards cell based immunotherapy for MM patients without the induction of GvHD. It also establishes the RAG2 ${ }^{-{ }^{-}} \mathrm{yc}^{-{ }^{-}-} \mathrm{MM}$ model as a clinically relevant platform to further develop NK cell based immunotherapy. 


\section{Introduction}

Multiple Myeloma ( $\mathrm{MM}$ ) is a hematological malignancy characterized by clonal expansion of plasma cells in the bone marrow ${ }^{1}$. Despite new options, state-of-the-art treatment results only in median survival of 5-7 years ${ }^{2}$ and new treatment strategies providing curative responses preferably at lower toxicity are urgently needed. Autologous-stem cell transplantation (auto-SCT) combined with high dose chemotherapy has improved overall survival as compared to treatment with conventional chemotherapy alone; still the great majority of patients eventually relapse ${ }^{3}$. Allogeneic-SCT (allo-SCT) has the potential to cure MM by the so called graft versus tumor response of donor immune cells. However, an important drawback of allo-SCT is the high occurrence of graft-versus-host disease (GVHD) mediated by donor $T$ cells responding to mismatched histocompatibility antigens $s^{4,5}$. In addition, treatment related mortality after allo-SCT is high and for patients surviving the procedure, there is still progression of disease in the majority of cases.

When allo-SCT is performed over HLA barriers, Natural Killer (NK) cells, in addition to T cells, can mediate GVM responses and an important advantage of NK cells is that they do not seem to mediate GvHD. NK cells are important immune effector cells, capable of targeting virally infected or malignantly transformed cells by means of a delicate balance of inhibitory and activating receptor signals ${ }^{7}$. An important class of inhibitory receptors is the killer immunoglobulin-like receptor (KIR) family interacting with the highly polymorphic HLA class I molecules expressed by virtually every healthy cell. The most important inhibitory KIRs are KIR3DL1 that binds HLA-Bw4 epitopes, KIR2DL2 and KIR2DL3 that interact with HLA-group C1 alleles and KIR2DL1 that binds HLA-group C2 alleles $^{8-10}$. Upon engagement of inhibitory KIRs with the HLA class I molecules of a target cell, inhibitory signals are transmitted, prohibiting NK cells to kill the target cell. Ruggeri et al. showed that the anti-tumor effect of NK cells in the haplo-identical SCT setting in acute myeloid leukemia (AML) could be attributed to the presence of "alloreactive NK cells" ${ }^{6}$. Alloreactive NK cells express KIRs for which the HLA ligand is missing in the patient. These KIR-ligand mismatched NK cells can kill tumor cells based on "missing self" principle if they have been licensed for the mismatched $\mathrm{KIR}^{11,12}$. This principle has been shown effective since AML patients treated with a KIR-ligand mismatched allogeneic bone marrow transplant show increased survival over patients transplanted without such a mismatch ${ }^{13}$.

In line with the AML data, beneficial effects of KIR-ligand allo-SCT on disease outcome have been reported for $\mathrm{MM}$ patients ${ }^{14}$. Unfortunately, since $\mathrm{MM}$ is a disease of the elderly, the majority of MM patients is not eligible for allo-SCT due to the severe conditioning regime and transplantation related complications. In a first study in MM patients, the clinical potential of isolated KIR-ligand mismatched NK cell infusion was tested in combination with high dose melphalan and fludaribine treatment and a delayed rescue with autologous stem cells ${ }^{15}$. Although the contribution of NK cells in 
addition to the conditioning regime could not be established, the trial encouragingly demonstrated the feasibility and safety of the approach. NK cells have also been shown to efficiently kill MM cells in vitro ${ }^{16-18}$. Recently, we showed that KIR-ligand mismatched NK cells are better in doing this than matched cells (Sarkar et al, chapter 3). We could demonstrate that this is also the case for MM cells under hypoxia, an immunosuppressive condition relevant for $\mathrm{MM}^{18}$. Together these data suggest that infusion of KIR-ligand mismatched NK cells is an interesting novel treatment option to provide curative effects bypassing the difficulties associated with a full HLA mismatched allo-SCT.

Immunodeficient mouse models provide an excellent opportunity for preclinical testing of novel treatment strategies. The $\mathrm{RAG}^{-/} \mathrm{Yc}^{-/-} \mathrm{MM}$ mouse model was originally developed by us to study GvHD development after injection of PBMCs or purified $\mathrm{T}_{\text {cells }}{ }^{19}$. In this model we previously showed that human PBMC xenografts did not only induce GvHD but also $\mathrm{GvM}^{20}$. In the present study, we used the RAG2 ${ }^{-1-\gamma \mathrm{C}^{-/}} \mathrm{MM}$ mouse model to investigate the effect of alloreactive KIR-ligand mismatched NK cells on GvM and GvHD in MM bearing mice.

\section{Materials and methods}

\section{Cell lines and cell cultures}

The human MM cell lines studied were U266, RPMI-8226/S, UM-9, LME-1, XG-1, OPM-1, and L-363, all retrovirally transduced with luciferase as described previously ${ }^{20}$. All MM cell lines were cultured in RPMI-1640 (Gibco, Breda, the Netherlands) except for XG-1 and LME-1, which were cultured in IMDM medium (Gibco), supplemented with $10 \%$ fetal calf serum (Integro, Zaandam, the Netherlands), $100 \mathrm{U} / \mathrm{ml}$ penicillin (Gibco), $100 \mu \mathrm{g} / \mathrm{ml}$ streptomycin (Gibco). The XG-1 cell line was additionally cultured in the presence of $20 \mu \mathrm{g} / \mathrm{ml}$ Transferine (Sigma) and $500 \mathrm{pg} / \mathrm{ml} \mathrm{IL-6}$ (Biosource). The cultures were maintained at $37^{\circ} \mathrm{C}$ with $5 \% \mathrm{CO} 2$ in humidified atmosphere. Presence and absence of HLA epitopes (HLA-C1, -C2 and -Bw4) and of HLA-A in cell lines (Table 4.1) and healthy control donors, were routinely determined by Sequence-Specific Oligonucleotides (SSO) analysis and Luminex ${ }^{\circledR}$ according to manufacturer's guidelines (One Lambda, Montpelier, France).

\section{Mice}

The RAG ${ }^{-1} \mathrm{Yc}^{-/-}$mice were originally obtained from the Amsterdam Medical Center (Amsterdam, the Netherlands). They were bred and maintained in cages under specified pathogen-free conditions at the Central Laboratory Animal Institute (Utrecht University, Utrecht, the Netherlands) and received sterile water and irradiated pellets ad libitum. All animal experiments were approved by the Ethical committee for Animal 
Experimentation of Utrecht University and in accordance with current Dutch Law on Animal Experiments.

\section{Induction of MM cells $\mathrm{RAG}^{-/-} \mathrm{\gamma c}^{-/-}$mice}

Induction of $\mathrm{MM}$ in $\mathrm{RAG2}^{-1-} \mathrm{Yc}^{-/-}$mice was performed as described in more detail $\mathrm{in}^{20}$. In brief, twenty-four hours before the injection of the freshly cultured MM cells, mice received total body irradiation (TBI; $3.0 \mathrm{~Gy}, 200 \mathrm{kV}$ X-rays). $5 \times 10^{6}$ U266 cells were injected intravenously (i.v.) via the lateral tail vein. For the experiments with subcutaneous (s.c.) MM tumors, $0.3 \times 10^{6}, 1 \times 10^{6}, 3 \times 10^{6}$ or $9 \times 10^{6}$ U266 cells in $100 \mu \mathrm{l}$ phosphate-buffered saline were injected s.c. in the flank of the mice. In all experiments tumor load was determined by bioluminescence imaging (BLI) measurements on ventral and dorsal site of the mice as described previously. The BLI images were analyzed with M3Vision from Biospace. In case of paralysis of the hind limbs or when the mice became moribund, they were sacrificed by cervical dislocation. To determine progression free survival (Figure 4.4 and 4.6) mean BLI signal (ventral BLI signal + dorsal BLI signal)/2 for each time point was calculated. Progression was defined as a mean BLI signal that was greater than $2 x$ the mean BLI signal at day 34 .

\section{Preparation and transplantation of huPBMCs and peripheral blood derived NK cells}

Buffy coats were obtained from healthy human blood donors that were HLA-genotyped (all donors were HLA-C1, C2 and Bw4 positive) and KIR genotyped (all donors expressed KIR2DL1, KIRDL2/3 and KIR3DL1) by Sequence-Specific Oligonucleotides (SSO) analysis and Lumine ${ }^{\circledR}$ according to manufacturer's guidelines (One Lambda). Mononuclear cells from peripheral blood of these healthy donors were isolated by density gradient separation. NK cells were either negatively (for NK cell groups) or positively (for NKdepleted group) selected by immunomagnetic cell separation according to manufacturer's protocol (Miltenyi Biotec $\mathrm{GmbH}$, Bergisch Gladbach, Germany). The purity of all isolated populations exceeded $95 \%$ as determined by flowcytometry. Unactivated NK cells were used directly upon isolation. For the experiments with IL-2 activated NK cells, the isolated NK cells were cultured overnight with $1000 \mathrm{U} / \mathrm{ml}$ of IL-2 (proleukin). Cord blood derived NK cells were expanded and differentiated into NK cells from umbilical cord blood CD34+ cells (HLA-C1, C2 and Bw4 positive) as described before $^{21,22}$ and were kindly provided by Dr. Spanholz and Dr. Groenewegen (Glycostem, Nijmegen, the Netherlands). Before injection, cells were counted and resuspended at the concentration indicated in the results section and legends. Suspensions of PBMC or NK cells were injected either intravenously into the mice via the tail vein (Figure 4.2, 4.4 and 4.6) or intratumorally (Figure 4.3). Mice received $0.2 \mathrm{ml}$ of the liposomal suspension intravenously one day before injection of the cells. Control mice received liposomes as well but no PBMC or NK cell infusion. 


\section{Flow cytometry}

Cell isolates from hind limbs were analyzed using flow cytometry for the presence of human cells in the total nucleated cell fraction. Bone marrow was obtained by flushing the bones with PBS. Single cell suspensions from the soft tissues were obtained by passaging through $70 \mu \mathrm{m}$ cell strainers (Becton Dickinson Biosciences, Erembodegem, Belgium). Fluorochrome conjugated monoclonal antibodies specific for surface molecules were used: CD3, CD45, CD45 CD56 all obtained from BD Biosciences. Cells were incubated with antibodies at proper dilutions for 30 minutes on ice. Samples were analyzed on a FACSCanto (BD Biosciences) with FACSDiva (BD) software.

\section{NK cell cytotoxicity assay}

Enriched NK cells, isolated as described in the preparation and transplantation of huPBMs section, were used in cytotoxicity assays. Target cells (MM cells) were labeled with 3'-dioctadecyloxacarbocyanine (DiO) according to the manufacturer's instructions (Sigma) and $2 \times 10^{4}$ target cells were incubated with NK cells at various effector:target $(\mathrm{E}: \mathrm{T})$ ratios for 12 hours, each ratio in triplicate. Percentage of killed target cells (\% PI positive and DiO positive cells) was determined by flowcytometry. Percentages specific lysis were calculated as follows: ((\% PI positive target cells - \% spontaneous PI positive cells)/ $\%$ of vital cells) $\times 100$.

\section{Statistics}

Differences in progression free survival (Figure 4.4 and 4.6) were calculated with Graphpad Prism software version 5.01 using log rank test (Mantel Cox) test.

\section{Results}

\section{Alloreactive NK cells kill MM cell lines in vitro}

To test whether freshly isolated alloreactive KIR-ligand mismatched NK cells could eliminate MM cells in vitro, NK cell alloreactivity against a panel of eight MM cell lines was determined. These cell lines were first genotyped for HLA-A,-B and-C to determine which KIR ligands were present and absent. Two of the cell lines, i.e. RPMI-8226/s and OPM-1, expressed HLA-C1 and -C2 but not the Bw4 epitope (Table 4.1). U266, LME-1, L-363 and UM-9 expressed only the HLA-C1 and not the C2 and BW4 epitopes. XG-1 was the only cell line expressing all three epitopes. Then, cell lines were co-cultured with freshly isolated NK cells derived from healthy donors. To avoid hyporesponsiveness of NK cells due to lack of licensing, donors expressing all three epitopes (i.e positive for HLA-C1, -C2 and -Bw4) were used. After 12 hours of co-culture, the percentage of death $\mathrm{MM}$ cells was determined by flowcytometric analysis of the percentage $\mathrm{PI}$ 
positive MM cells. This revealed that for LME-1, L363, LME-1 and U266 specific lysis was $60-70 \%$ (Figure $4.1 \mathrm{~A}-\mathrm{B}$ ) demonstrating that these cell lines were almost as sensitive to KIR-ligand mismatched NK cells as the K562, a leukemia cell line devoid of HLA class I expression that was used as positive control cell line (Figure 4.1D). For RPMI-8226/s and UM-9, the specific lysis at 20:1 E:T ratio was $15 \%$ and $25 \%$ respectively indicating that these cell lines were relatively resistant to alloreactive NK cells (Figure 4.1A-B). For the $\mathrm{C} 1+\mathrm{C} 2+\mathrm{Bw} 4+$ cell line $X \mathrm{X}-1$ this percentage was even lower, i.e. 10\% (Figure 4.1C). Together this shows that MM cell lines, though at a different efficiency can by killed by alloreactive NK cells in vitro.

\section{Freshly isolated, resting alloreactive NK cells can mediate anti-MM effects in vivo}

To investigate whether NK cells mediate anti-MM responses in vivo, the RAG2/ $\mathrm{\gamma c}^{-/-}$ mouse disseminated MM model was used with the U266 cell line. This cell line was sensitive for NK cells in vitro (Figure 4.1) and in a previous study we showed that infusion of PBMC could cure mice from established MM induced by injection of U266 cells $^{20}$. In the current study, $5 \times 10^{6}$ U266 cells were i.v. injected on day 0 followed by i.v. injection of either PBMC or freshly isolated, resting NK cells at different doses on day 21, 23 and 25. To test whether NK cells were required for the anti-tumor response after PBMC injection, one group of mice received PBMC depleted from NK cells. Both PBMC and NK cells were obtained from peripheral blood of donors expressing all three KIR ligands (i.e. HLA-C1, -C2 and-Bw4). Monitoring of tumor growth by BLI revealed that all treatment groups had developed MM at the day of PBMC or NK cell injection (Figure 4.2A-B). As expected from our previous studies, injection of PBMC subsequently completely cured the mice from MM. NK cells were not required for this curative effect as injection of NK cell depleted PBMC was as effective as injection of the complete PBMC fraction.

NK cell treatment was performed by three times injection with either $2 \times 10^{6}, 4 \times 10^{6}$ or $8 \times 10^{6}$ NK cells per injection. In none of the two groups receiving the lower NK cell dose this resulted in a robust anti-MM response and tumor load of the NK cell groups was comparable to the tumor load in the control group (i.e. group w.o. PBMC or NK cells; Figure 4.2A-B). However, in the group receiving the highest dose of NK cells one mouse did show a reduction in tumor burden upon infusion of NK cells suggesting that NK cells can mediate anti-MM responses in this model (Figure $4.2 \mathrm{C}$ ). In the groups receiving PBMC or NK cell depleted PBMC all mice developed signs of GvHD as observed by visual inspection of the mice and by weight loss. Importantly, none of the NK cell treated mice showed signs of GvHD demonstrating the safety of NK cell infusion. 
A

$\mathrm{C1}^{+} \mathrm{C2}^{+}{ }^{\mathrm{Bw}} 4^{-}$

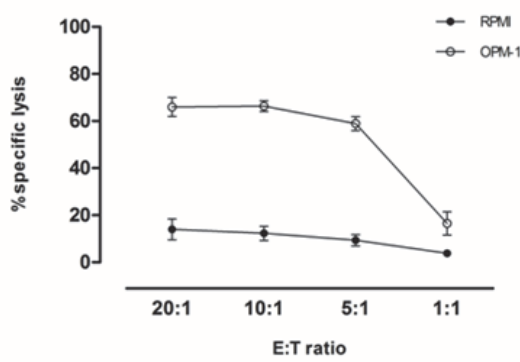

C
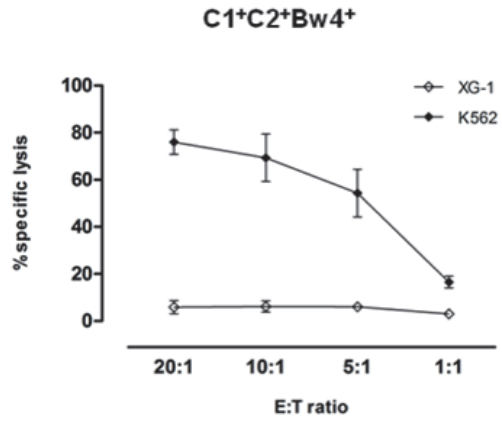

B

$\mathrm{C}^{+} \mathrm{C} 2{ }^{-B w} 4^{-}$

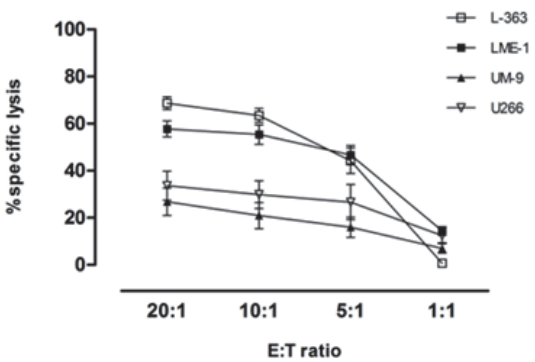

Figure 4.1 Allogeneic NK cells kill MM cell lines in a cell line dependent manner in vitro.

NK cells were isolated from healthy donors positive for all three KIR epitopes (HLA-C1, -C2, and -Bw4) and were co-cultured with DiO labeled MM cells at 1:20, 1:10 1:5 and 1:1 target:effector ratio. After 12 hours, the percentage of death (PI positive) MM cells was determined by flowcytometry. Graphs depict means and SD of specific lysis with six donors. A) Specific lysis of MM cell lines expressing HLA-C1 and $-\mathrm{C} 2$ but not Bw4 epitopes, B) MM cell lines expressing HLA-C1 but not C2 and Bw4 epitopes and C) MM cell line expressing all three NK cell epitopes and specific lysis of the positive control leukemia cell line K562, lacking surface expression of HLA-ABC. 
A

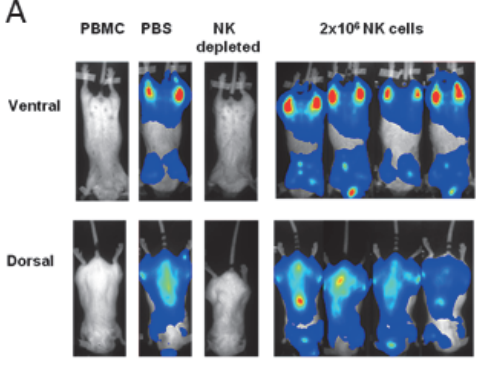

$4 \times 10^{6} \mathrm{NK}$ cells

$8 \times 10^{5} \mathrm{NK}$ cells
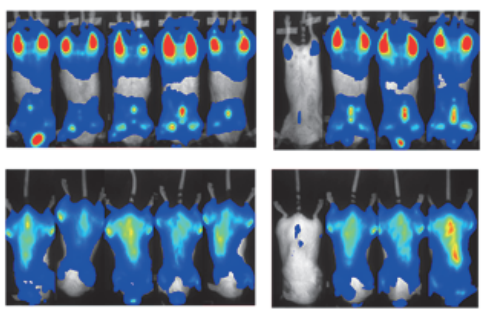

B

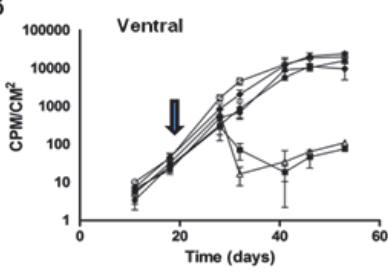

C
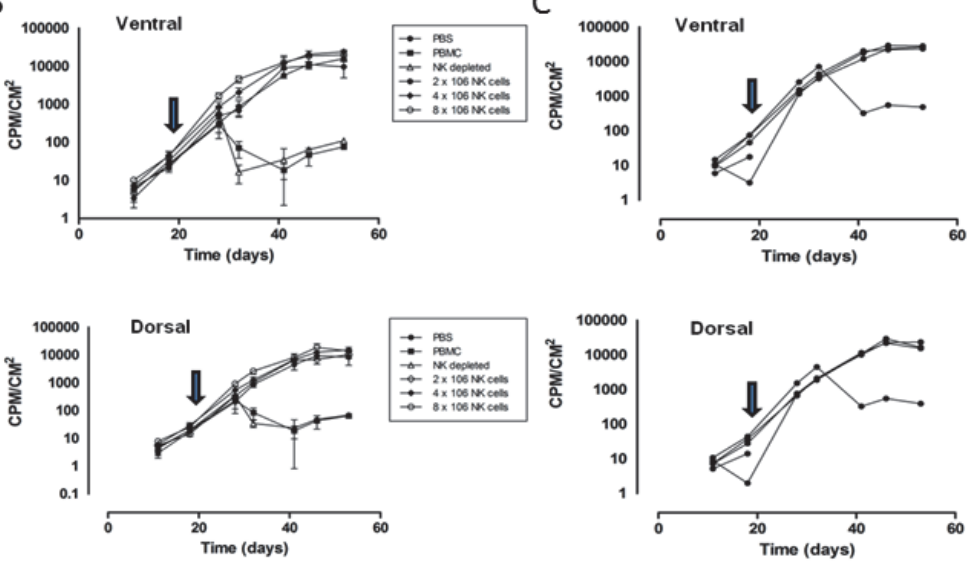

Figure 4.2 Freshly isolated, resting allogeneic NK cells can mediate anti-MM effects in vivo.

At day $0,5 \times 10^{6}$ U266 cells were i.v. injected into $\mathrm{RAG2}^{-1} \mathrm{\gamma c}^{-1-}$ mice. On day 20 , mice received one injection with liposomes followed on day 21,23 and 25 by i.v. injection of either $8 \times 10^{6}$ PBMC or freshly isolated NK cells $\left(2 \times 10^{6}, 4 \times 10^{6}\right.$ or $8 \times 10^{6} \mathrm{NK}$ cells per injection). One group received three injections with NK cell depleted PBMC. PBMC and NK cells were obtained from donors expressing all three KIR ligands (i.e HLA-C1, $-\mathrm{C} 2$ and $-\mathrm{Bw} 4$ ). Tumor growth was monitored by BLI of the ventral and dorsal side. A) BLI images obtained on day 53 of representative mice of the control, PBMC and NK cell depleted PBMC groups and of individual mice of the NK cell groups. B) graphs show per treatment group mean and SD of the BLI signal measured on the indicated days. C) Graphs show BLI data per mouse for the group receiving three injections of $4 \times 10^{6} \mathrm{NK}$ cells. Arrows in the graphs in B and C indicate the time of PBMC or NK cell infusion.

\section{IL-2 activated NK cells mediate anti-MM responses in a dose dependent manner in vivo}

We have previously shown that IL-2 activation can enhance the anti-MM response of NK cells in vitro ${ }^{18}$. To examine whether IL-2 activation could also improve the anti-MM response in vivo, U266 MM cells $\left(0.3 \times 10^{6}, 1 \times 10^{6}, 3 \times 10^{6}\right.$ or $9 \times 10^{6}$ U266 cells) were mixed with $1 \times 10^{6} \mathrm{NK}$ cells followed by immediate s.c. injection in the flank of RAG2 ${ }^{-1-} \mathrm{\gamma c}^{-/-}$mice. Monitoring of tumor growth showed that the BLI signal increased between day 0 and 
day 9 in the groups of mice receiving any of the four doses of U266 cells but not NK cells (Figure 4.3A). This increase in BLI signal was also observed for the groups receiving U266 cells and non-activated, resting NK cells. For all groups injected with U266 cells and IL-2 activated NK cells the BLI signal was lower than that of the corresponding control groups and in the group receiving the lowest number of U266 cells the effect was already detectable by in vivo imaging on day 1 . Co-injection of activated NK cells prohibited outgrowth of the tumor (Figure 4.3A).

The above-mentioned data demonstrated that the effect of IL-2 activated NK cells occurred in a dose dependent manner. To further investigate this and to investigate whether IL-2 activated NK cells could eliminate established MM in vivo as well, mice were s.c. injected with either $0.3 \times 10^{6}, 1 \times 10^{6}, 3 \times 10^{6}$ or $9 \times 10^{6}$ U266 cells. Upon development of a s.c. tumor on day 13, IL-2 activated NK cells were injected intratumorally. In the two groups receiving the highest number of U266 cells $\left(3 \times 10^{6}\right.$ or $9 \times 10^{6}$ cells) NK cell injection did not reduce the tumor load (Figure 4.3B). However, in the two groups receiving the lowest number of $U 266$ cells $\left(0.3 \times 10^{6}\right.$ or $1 \times 10^{6}$ cells) a reduction in the BLI signal could be observed after NK cell injection. Together these data demonstrate that IL-2 activated NK cells can mediate anti-MM responses in vivo but that the effector to target ratio is very important.

\section{IL-2 activated human NK cells can home to the bone marrow of MM bearing RAG2 ${ }^{-1-} \mathrm{\gamma C}^{-1-}$ mice}

Because IL-2 activated NK cells seemed more effective in eliminating s.c. MM than non-IL-2 activated NK cells we next investigated the effect of IL-2 activated KIR-ligand mismatched NK cells in the disseminated MM model. To this end, $5 \times 10^{6}$ U266 cells were i.v. injected followed, on day 21, 23 and 25, by i.v. injection of PBMC ( $n=4$ mice) or $8 \times 10^{6} \mathrm{NK}$ cells that were overnight activated with IL-2 ( $\mathrm{n}=6$ mice). As an alternative source of activated NK cells we used NK cells expanded from cord blood and six mice received on day 21 one injection with $24 \times 10^{6}$ cord blood derived NK cells. To promote NK cell survival, IL-15 was injected i.p. three times a week for two weeks after NK cell infusion. It has been previously shown that IL-15 administration alone promotes in vivo survival and expansion of NK cells while it does not have an anti-tumor effect ${ }^{23}$. 
A
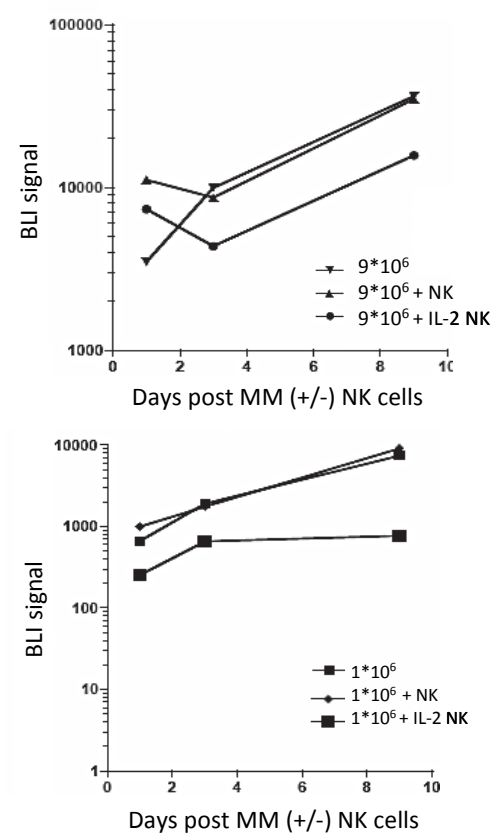
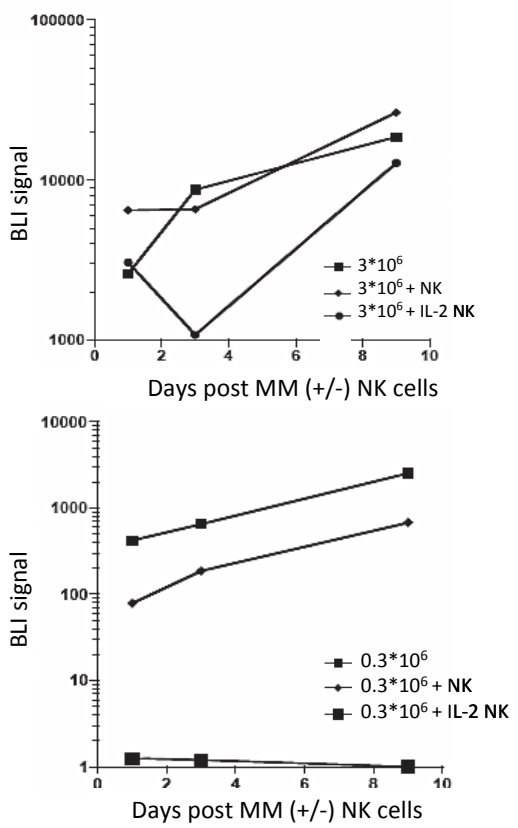

B

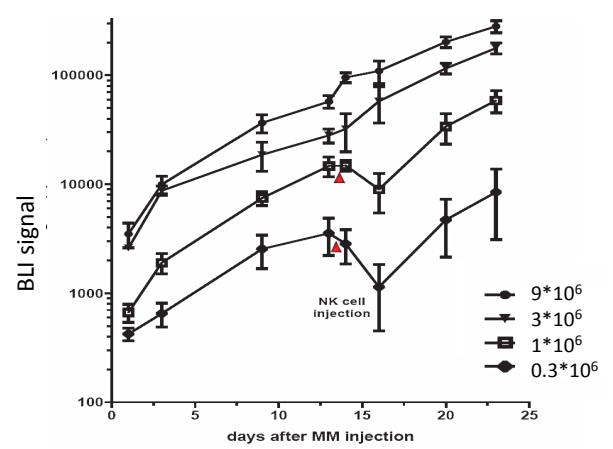

Figure 4.3 IL-2 activated NK cells mediate anti-MM responses in vivo in a dose dependent manner.

A) U266 MM cells $\left(0.3 \times 10^{6}, 1 \times 10^{6}, 3 \times 10^{6}\right.$ or $9 \times 10^{6}$ U266 cells) were mixed with $1 \times 10^{6} \mathrm{NK}$ cells. NK cells were either freshly isolated or activated overnight with $1000 \mathrm{U} / \mathrm{ml}$ of IL-2. Immediately after mixing cells were injected s.c. in the flank of RAG2 ${ }^{-1} \gamma c^{-1-}$ mice. Control mice received U266 cells without NK cells. Tumor growth was monitored on the indicated days by BLI. Graphs show the mean BLI signal of $n=3$ mice per treatment group (no NK cells, unactivated, resting NK cells and activated NK cells). Each graph depicts one dose of U266 cells. B) RAG2 $2^{-1} \gamma \mathrm{c}^{-1-}$ mice were s.c. injected with either $0.3 \times 10^{6}, 1 \times 10^{6}, 3 \times 10^{6}$ or $9 \times 10^{6} \mathrm{U} 266$ cells. NK cells were overnight activated with $1000 \mathrm{U} / \mathrm{ml} \mathrm{IL-2}$ and were injected intratumorally on day 13. Tumor was monitored at the indicated days by BLI. Graph shows mean BLI signal per group of $n=3$ mice. Arrow indicates the time of NK cell injection. 
Around day 40, all control mice (mice receiving no NK cells or PBMC) were sacrificed due to high tumor burden. For the NK cell treated mice progression free survival was slightly longer than for control mice ( $P=0.02$; Figure 4.4A). Nevertheless, also the NK cell treated mice died due to high tumor load. In line with our experiment in Figure 4.2, injection of PBMC reduced tumor burden but these mice had to be sacrificed due to severe GvHD around day 50. Symptoms of GvHD were not observed in NK cell treated mice. Importantly, flowcytometric analysis of the bone marrow harvested at the day of sacrifice of the mice revealed that cells expressing the human leukocyte marker CD45 and CD56 but not CD3 ( $\mathrm{hCD} 45+\mathrm{CD} 56+\mathrm{CD} 3$ - is indicative of NK cells) were present in the bone marrow (Figure 4.4B). These hCD45+CD56+CD3- NK cells were only present the IL-2 activated NK cell treated groups indicating that NK cells could actually home to the bone marrow. In the mice receiving NK cells obtained from peripheral blood hCD45+CD56-CD3+ T cells and cells expressing hCD45+ but not CD56 or CD3 were also present in the bone marrow.

\section{Cyclophosphamide (cyclo)/Total Body Irradiation (TBI) treatment alone does not cure disseminated MM}

In parallel studies in breast cancer mouse models we obtained evidence that cyclo/TBI conditioning is important for in vivo anti-tumor responses of alloreactive NK cells against $4 \mathrm{~T} 1$ breast cancer tumor ${ }^{24}$. In the current study, we therefore aimed to investigate whether addition of cyclo/TBI to the treatment regime resulted in prolonged progression free survival in the RAG2 ${ }^{-/-} \mathrm{Yc}^{-/-} \mathrm{MM}$ model To confirm that cyclo/TBI treatment was performed at a dosage that by itself was not sufficient to eliminate the tumor, we performed a pilot experiment in which RAG2 ${ }^{-1-} \mathrm{Yc}^{-/-}$mice were i.v. injected with $5 \times 10^{6}$ U266 cells on day 0 . After development of MM, mice were irradiated with 2 Gy on day 34 and day 35 . On day 35 mice also received $50 \mathrm{mg} / \mathrm{kg}$ cyclophospamide. Monitoring of tumor growth revealed that although cyclo/TBI treatment initially reduced the tumor burden, it was not sufficient to cure the mice as the tumor started growing again in these mice (Figure 4.5). 
A

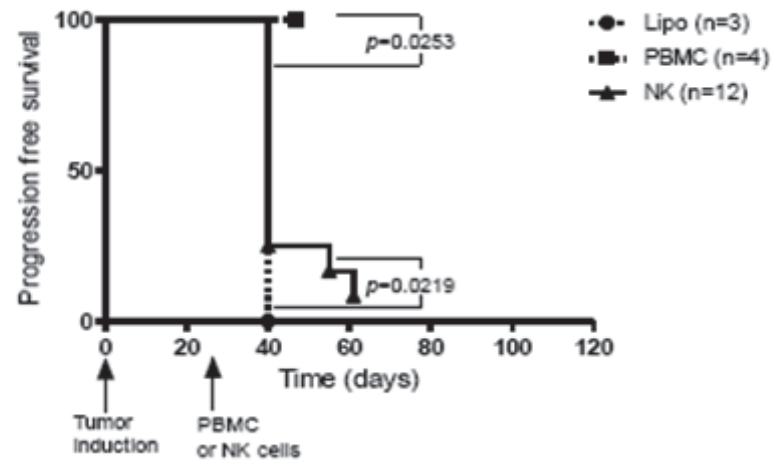

B
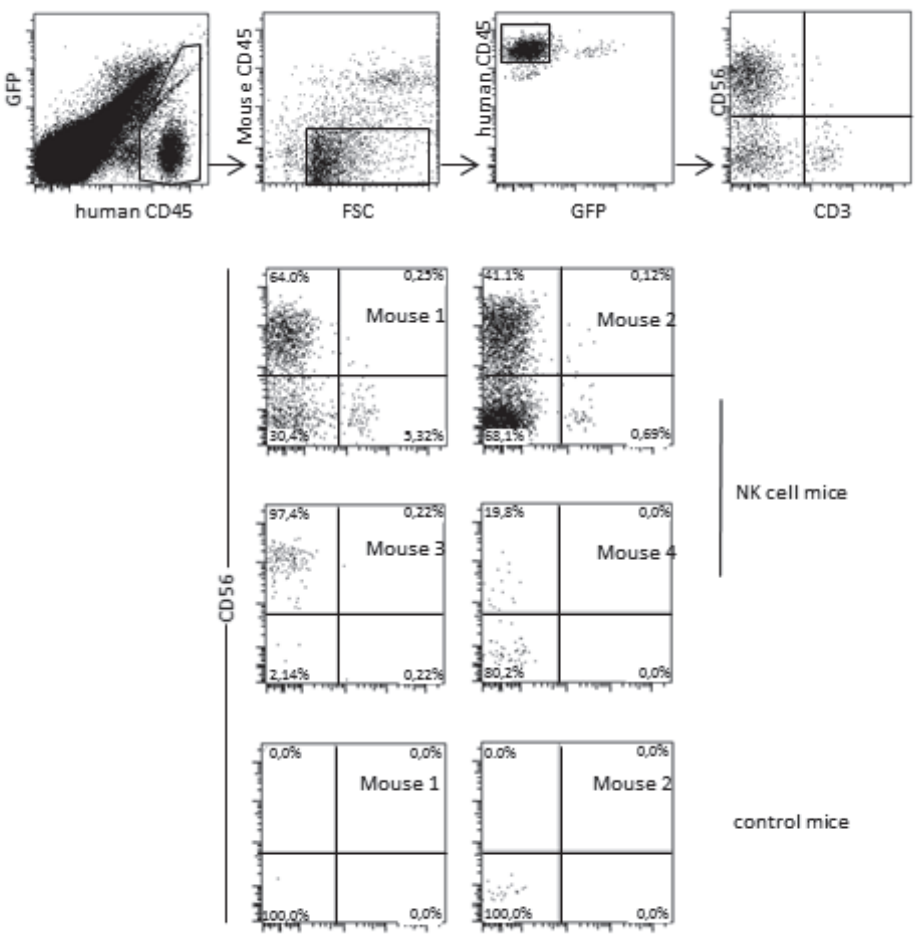

NK cell mice

control mice

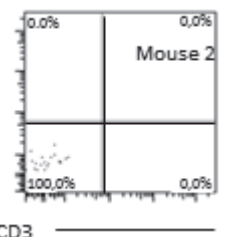

Figure 4.4 IL-2 activated NK cells can home to the bone marrow of MM bearing RAG2 ${ }^{-1-} \mathrm{yc}^{-/-}$mice.

At day $0,5 \times 10^{6}$ U266 cells were i.v. injected into $\mathrm{RAG2}^{-/} \mathrm{Yc}^{-/-}$mouse. On day 20 mice received one injection with liposomes followed on day 21,23 and 25 by i.v. injection of $8 \times 10^{6}$ PBMC or NK cells that were overnight activated with $1000 \mathrm{u} / \mathrm{ml} \mathrm{IL-2.} \mathrm{Tumor} \mathrm{growth} \mathrm{was} \mathrm{monitored} \mathrm{by} \mathrm{BLI}$ of the ventral and dorsal side. A) Graph shows progression free survival per treatment group. days. To determine progression free survival the mean BLI signal (ventral BLI signal + dorsal BLI signal)/ 2 for each time point was calculated. Progression was defined as a mean BLI signal that was greater than $2 x$ the mean $B L I$ signal at day 34 . B) Bone marrow was obtained at the day of sacrifice of the mice. Homing of human leucocytes to the bone marrow was analyzed by flowcytometry. Plots show representative gating strategy and examples of four NK cell treated mice and two control mice receiving only liposomes but not NK cells or PBMC. 
A

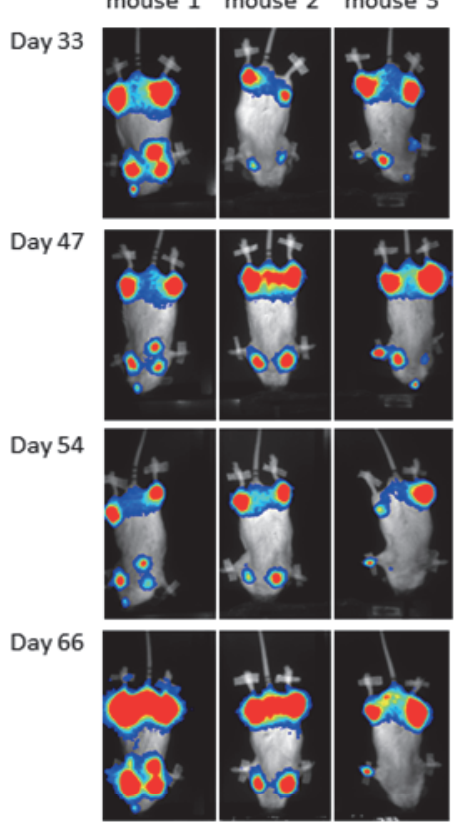

B

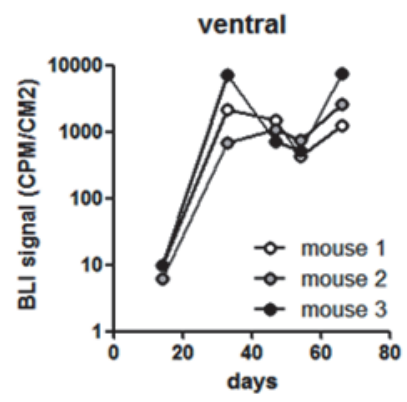

dorsal

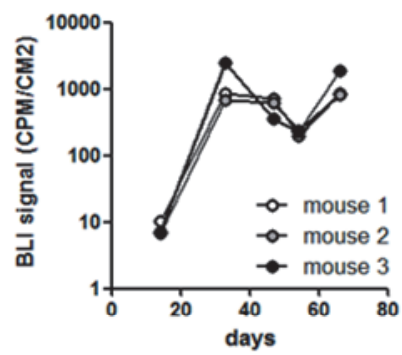

Figure 4.5 Cyclo/TBI treatment alone does not cure disseminated MM

RAG2 ${ }^{-1} \mathrm{Yc}^{-/-}$were i.v. injected with $5 \times 10^{6}$ U266 cells on day 0 . On day 34 and 35 , mice were irradiated with 2 Gy. On day 35, mice also received an injection with $50 \mathrm{mg} / \mathrm{kg}$ cyclophosphamide. Tumor growth was monitored by BLI on both ventral and dorsal sides. A) Dorsal BLI images from the mice at the indicated days. B) Graph shows BLI signal per mouse on the indicated days.

\section{Combination treatment with cyclo/TBI and IL-2 activated NK cells can provide anti-MM response in the disseminated $\mathrm{MM}$ model}

To investigate the effect of treatment with IL-2 activated NK cells in combination with cyclo/TBI, MM was induced in RAG2 $2^{-1} \mathrm{yc}^{-/-}$mice by i.v. injection of $5 \times 10^{6}$ U266 cells on day 0 . In line with our pilot, cyclo/TBI treatment was done at days 34 and 35 and tumor growth was monitored by BLI. This revealed that, in agreement with our pilot, cyclo/TBI treatment only slightly and not significantly, increased progression free survival as compared to control mice $(P=0.06$; Figure 4.6). However, chemo/radio therapy in combination with injection of $4-8 \times 10^{6} \mathrm{IL}-2$ activated blood derived NK cells, on day 36 , 38 and 42 , or $24 \times 10^{6}$ of cord blood-derived NK cells on day 36 prolonged progression free survival as compared to control mice ( $P=0.003$; Figure 4.6). Two mice in the NK cell treated group died for unknown reason while having a very low tumor load and without having signs of GVHD. Also in the other NK cell treated mice we did not observe signs of GvHD by visual inspection. Although MM eventually progressed, this shows that 
combination treatment with cyclo/TBI and IL-2 activated alloreactive NK cells can provide anti-MM effects against established disseminated MM.
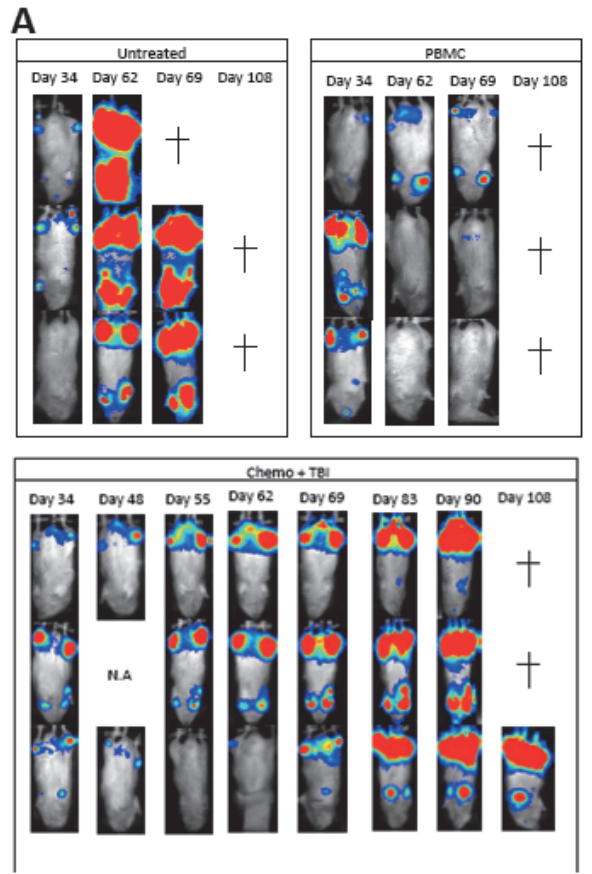

B
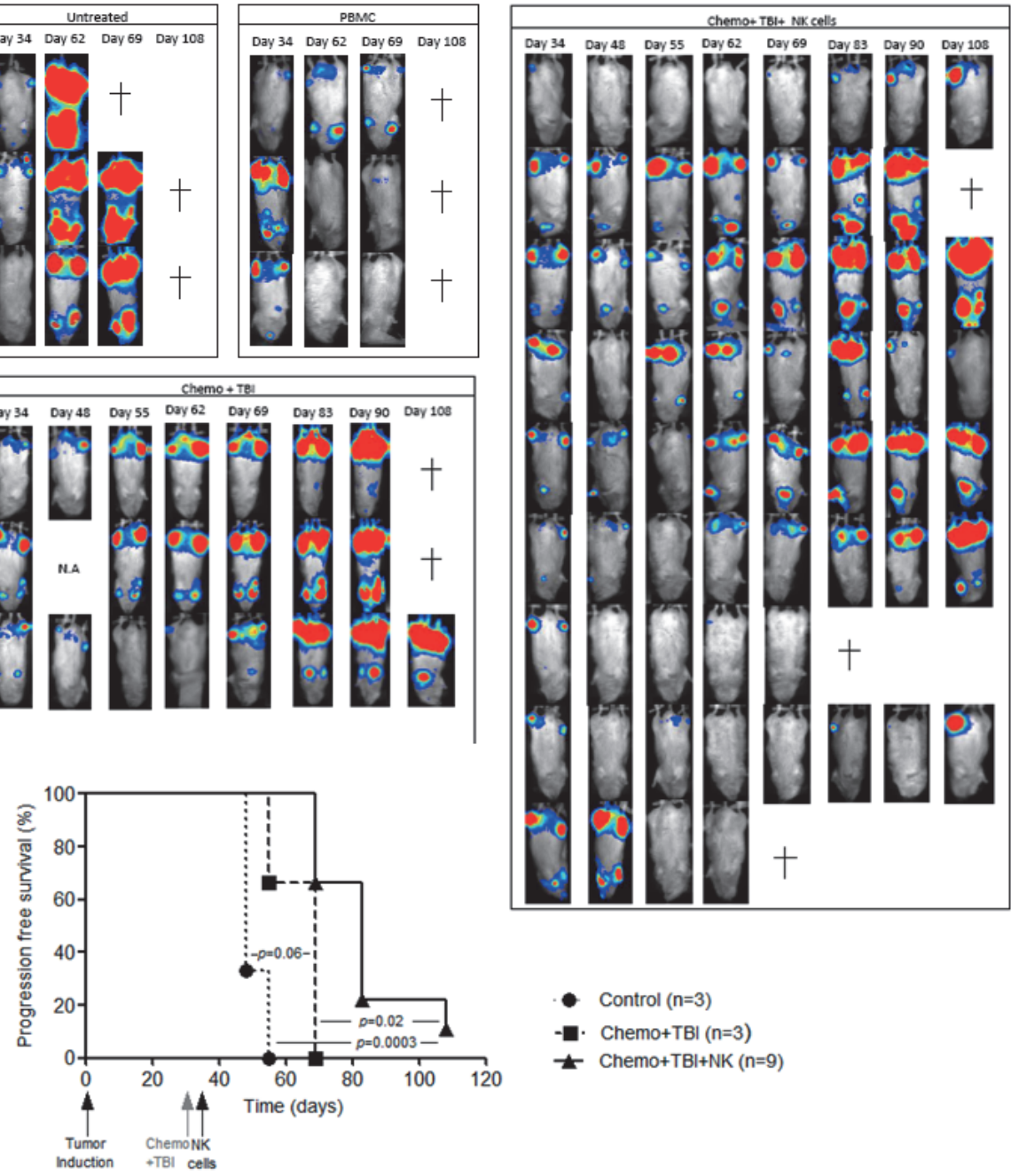

Figure 4.6 Combination treatment with cyclo/TBI and IL-2 activated NK cells provides anti-MM responses in the disseminated model.

RAG $^{-1} \mathrm{Yc}^{-1-}$ were i.v. injected with $5 \times 10^{6}$ U266 cells on day 0 . On day 34 and 35 , mice were irradiated with $2 \mathrm{~Gy}$. On day 35, mice also received an injection with $50 \mathrm{mg} / \mathrm{kg}$ cyclophosphamide. NK cell treated mice also received activated NK cells (blood or cord blood derived) on days 36,38 and 42 by i.v. injection. Control mice received neither cyclo/TBI nor NK cells. Tumor growth was monitored by BLI on both ventral and dorsal sides. A) Dorsal BLI images from the mice at the indicated days. B) Graph shows progression free survival per treatment group. To determine progression free survival mean BLI signal (ventral BLI signal + dorsal BLI signal)/2 for each time point was calculated. Progression was defined as a mean BLI signal that was greater than $2 x$ the mean BLI signal at day 34 . 


\section{Discussion}

Despite novel treatment options, MM remains largely incurable illustrating the need to develop novel therapeutic treatment means. In the present study, we investigated the effect of alloreactive KIR-ligand mismatched NK cells on GvM and GvHD in the $\mathrm{RAG}^{-1-} \mathrm{Yc}^{-/-}$mouse MM model. This revealed that IL-2 activated NK cells can mediate anti-MM responses in combination with low dose conditioning with cyclo/TBI. Importantly, we did not observe signs of GVHD in any of the experimental treatment protocols upon NK cell injection. In line with these data, a first clinical study in MM showed that KIR-ligand mismatched NK cells could be infused into MM patients without causing toxicity and $\mathrm{GVHD}^{15}$ and comparable observations have been made in AML. Altogether this illustrates the feasibility and clinical potential of the approach.

Thus far, the clinical efficacy of NK cell infusion in cancer patients has been somewhat inconsistent emphasizing that optimization of therapeutic protocols is required ${ }^{25}$. NK cells can kill only a limited number of target cells. In the current study, we observed that NK cell responses against s.c. MM occurred in a NK cell dose dependent manner (Figure 4.3) and with unactivated, resting NK cells we only observed a biological effect of NK cells in one of the mice with disseminated MM in the group receiving the highest NK cell dose (Figure 4.2). Dose dependency has also been shown in the 5T33M MM model were the anti-MM response of transplanted mouse NK cells could be enhanced by infusion of a higher number of NK cells ${ }^{26}$. In another study, with human NK cells expanded on K562-mb15-41BBL feeder cells, adoptive transfer of $160 \times 10^{6} \mathrm{NK}$ cells inhibited growth of OPM2 while transfer of $40 \times 10^{6} \mathrm{NK}$ cells was not sufficient ${ }^{27}$. These data suggest that infusion of really high numbers of NK cells are needed to improve clinical efficacy of NK cell therapy.

In our model, mice having established MM were treated with NK cells. In that way, our model resembles the clinical situation were tumor burden will be relatively high and MM patients, even patients in remission, can bear up to $10^{9}$ tumor cells. Because of this high tumor load, the number of NK cells required for clinically effective therapy will presumably be higher than the number that can be obtained by isolation from peripheral blood. Ex vivo expanded NK cells could provide a solution and are an interesting alternative for NK cells derived from apheresis products. An additional advantage is that the ex vivo culturing period would allow for extra NK cell activation. In the current study, we used two sources of NK cells; IL-2 activated NK cells isolated from blood of healthy individuals and NK cells that were generated and expanded from cord blood $^{21,22}$. With both sources we observed reactivity against established, disseminated $\mathrm{MM}$ in combination with chemo/TBI (Figure 4.6). This is in line with two previous studies showing that NK cells expanded on K562-mb15-41BBL feeder cells ${ }^{27}$ and the NK 
cell lines NK-92 or KHYG- ${ }^{28}$ reduced disease burden in vivo together demonstrating that different sources of NK cells could be further tested for clinical application.

In the current study, NK cell donors were selected based on the genotypic presence of a mismatch between inhibitory KIRs on the NK cells and the HLA epitopes on the U266 cell line. Phenotypically however, the infused NK cell population was heterogeneous because NK cells express one or a combination of inhibitory receptors on their cell surface hence leading to different NK cells subsets; i.e. subsets exclusively expressing KIRs mismatched with the HLA ligands present on U266 target cells, subsets exclusively expressing KIRs matched with the ligands, subsets expressing both types of KIRs and a subset expressing none of the KIRs. Therefore, only a small percentage of the infused NK cells exclusively expressed KIRs mismatched with the U266 HLA ligand (HLA-C1) and the majority of NK cells (co-)expressed NKG2A. We recently showed, in an in vitro study, that KIR-ligand mismatched NK cells are more effective than matched NK cells in mediating anti-MM responses (Sarkar et al., chapter 3). In addition, we demonstrated that U266 cells in this disseminated MM model expressed HLA-E at a level that is sufficient to inhibit NKG2A expressing NK cells (Sarkar et al., chapter 3). Based on these data NK cells expressing mismatched KIRs and lacking expression of matched KIRs or NKG2A could be expected to be the most potent effector cells against $M M$ in vivo as well. Future experiments with HLA-E knockout Luc expressing U226 in the RAG2 ${ }^{-1} \mathrm{Yc}^{-/-}$ mouse would enable us to exactly determine the detrimental effect of HLA-E during NK cell therapy. Ruggeri et al. have demonstrated that, in an AML mouse model, indeed the mismatched NK cells mediated in vivo anti-tumor effects while matched NK cells did not $^{6}$. Therefore, it is highly relevant to study whether KIR-ligand mismatched NK cells are indeed more potent against $\mathrm{MM}$ in vivo because acquiring high enough numbers of these subsets for clinical application will be challenging and it is probably worthwhile to develop strategies to promote expansion or development of NK cell subsets with the highest anti-MM reactivity.

Our data show that U266 cells are highly sensitive for NK cell killing in vitro (Figure 4.1) while in vivo these cells seemed to be more difficult to eliminate. In addition to numbers and the small numbers of KIR-ligands mismatched NK cells, an explanatory factor could be that only a fraction of the NK cells actually homes to the bone marrow resulting in a less favorable E:T ratio as compared to in vitro. Although the experiments were not designed for detailed examination of the homing capacity of NK cells, phenotypic analysis of bone marrow cell at the time of sacrifice of the mice revealed that activated NK cells derived from peripheral blood and cord blood can home to the tumor site in our current model (Figure 4.4). This is in line with a previous study showing that cord blood derived NK cells migrate to the bone marrow of NSG mice and functionally express CXCR4 ${ }^{23}$, a chemokine receptor described to be important for homing to the bone marrow ${ }^{29}$, and with the observation that primary blood derived NK 
cells express $\mathrm{CXCR}^{30}{ }^{30}$. In addition to NK cells, we observed some $\mathrm{T}$ cells (huCD45+CD3+CD56-) and human leukocytes that were neither NK cells nor T cells (huCD45+CD3-CD56-) in mice receiving NK cells from peripheral blood. Presumably this was the result of the minor impurity of the peripheral blood derived NK cell product. Although the contaminating $T$ cells in the peripheral blood product could mediate antiMM activity as well, the number of $T$ cells contaminating the products was not sufficient to explain the anti-MM response. To induce a xenogeneic GVH with infused PBMC cells at least $6 \times 10^{6} \mathrm{~T}_{\text {cells }}{ }^{40}$ are required in pre-conditioned mice (TBI + toxic liposomes). In the experiment where we combined cyclo/TBI and NK cell treatment we did not observe any human lymphocytes in the bone marrow at the time of sacrifice which presumably was due to the longer follow up period 108 days vs 60 days in the experiment with activated NK cells without cyclo/TBI.

In the RAG2 ${ }^{-1} \mathrm{Yc}^{-/-}$model, $\mathrm{MM}$ cells grow in their natural environment, i.e. the bone marrow, making it an excellent model for preclinical testing of immunotherapy reflecting MM pathophysiology. This is important because it is exactly this environment that, through the presence of factors like hypoxia ${ }^{18}$ and stromal cells ${ }^{31}$ or soluble factors like PGE2 ${ }^{32}$ can suppress development of effective anti-tumor immunity making it extremely important to test the anti-MM capacity of NK cells in such relevant models. An interesting other preclinical model recently developed by our group is a scaffoldbased model where patient derived MM cells grow on s.c. implanted scaffolds $s^{33}$. In such models, it would be highly interesting to address the synergistic potential of a combination of approaches targeting immunosuppresive bone marrow associated factors with alloreactive NK cell therapy to achieve maximal efficiency of the response. One such approach can be the combination of chemo/radiotherapy and activated NK cells that, in our study, resulted in a better anti-MM response as compared to treatment with activated NK cells alone. Here, we did not study the mechanism by which the conditioning regime enhanced NK cell functioning but, several previous studies demonstrated that either chemo or radiotherapy can render tumor cells more susceptible for NK cells by upregulation of activating ligands ${ }^{34-36}$. On the other hand, the conditioning could trigger the release of cytokines NK cell promoting cytokines such as IL-2, IL-15 and IL- $21^{37}$ or promote recruitment or survival of the NK cells at the tumor site ${ }^{38,39}$. Also, it would be interesting to combine immunomodulatory drug Pomalidomide or ADCC triggering agents such as Elotuzumab to boost the NK cell activity in this model.

In the current study we showed the biological anti-MM potential of alloreactive NK cells against disseminated MM. In addition, we demonstrate that IL-2 activated NK cells can home to the bone marrow of $\mathrm{MM}$ bearing mice and that anti-MM responses occurred in a dose dependent manner. The latter suggests that infusion of a higher number of alloreactive NK cells can help to improve clinical efficacy and that patients should, 
preferably, be treated in remission or at least with low residual disease. Our data also demonstrate that combination therapy of alloreactive NK cells and chemo/radiotherapy

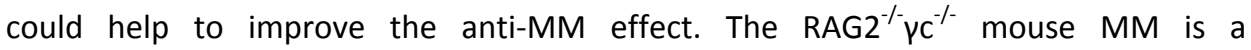
pathophysiologically relevant model to further develop and optimize NK cell treatment protocols and to test ex vivo expanded/activated NK cells ultimately leading to enhanced clinical efficacy.

\section{Acknowledgements}

The RAG ${ }^{-1} \mathrm{YC}^{-1-}$ mice were originally obtained from the Amsterdam Medical Center (AMC, Amsterdam, The Netherlands). The myeloma cell line LME-1 was kind gift from Dr. R. van Oers, AMC, Amsterdam, The Netherlands. The authors would like to thank the tissue typing laboratory (MUMC+) for HLA typing of the cell lines. The authors would also like to thank Dr. Dirk Groenewegen and Dr. Jan Spanholz (Glycostem technologies, Nijmegen) and Dr. Harry Dolstra (Radbout UMC) for providing cord blood derived NK cells. The authors would also like to thank Regina de Jong-Korlaar and Frans Hofhuis Department of Cell Biology, University Medical Center Utrecht for their technical assistance with animal studies. S. Sarkar was supported by a PhD grant from GROW, School of Oncology and Developmental Biology. L. Wieten was supported by a personal grant from Dutch Cancer association (KWF kankerbestrijding; UM2012-5375). G. Bos, M. van Gelder and L. Wieten were supported by a grant from the Kankeronderzoeksfonds Limburg. 


\section{REFERENCES}

1. Raab MS, Podar K, Breitkreutz I, Richardson PG, Anderson KC (2009) Multiple myeloma. Lancet 374: 324-339.

2. Palumbo A, Anderson K Multiple myeloma. N Engl J Med 364: 1046-1060.

3. Bensinger WI (2009) Role of autologous and allogeneic stem cell transplantation in myeloma. Leukemia 23: $442-448$.

4. Lokhorst HM, Schattenberg A, Cornelissen JJ, Thomas LL, Verdonck LF (1997) Donor leukocyte infusions are effective in relapsed multiple myeloma after allogeneic bone marrow transplantation. Blood 90 : 4206-4211.

5. Shlomchik WD (2007) Graft-versus-host disease. Nat Rev Immunol 7: 340-352.

6. Ruggeri L, Capanni M, Urbani E, Perruccio K, Shlomchik WD, et al. (2002) Effectiveness of donor natural killer cell alloreactivity in mismatched hematopoietic transplants. Science 295: 2097-2100.

7. Vivier E, Ugolini S, Blaise D, Chabannon C, Brossay L (2012) Targeting natural killer cells and natural killer T cells in cancer. Nat Rev Immunol 12: 239-252.

8. Colonna M, Borsellino G, Falco M, Ferrara GB, Strominger JL (1993) HLA-C is the inhibitory ligand that determines dominant resistance to lysis by NK1- and NK2-specific natural killer cells. Proc Natl Acad Sci U S A 90: 12000-12004.

9. Moretta L, Locatelli F, Pende D, Marcenaro E, Mingari MC, et al. (2011) Killer Ig-like receptor-mediated control of natural killer cell alloreactivity in haploidentical hematopoietic stem cell transplantation. Blood 117: 764-771.

10. Parham P (2005) MHC class I molecules and KIRs in human history, health and survival. Nat Rev Immunol 5: 201-214.

11. Jonsson AH, Yokoyama WM (2009) Natural killer cell tolerance licensing and other mechanisms. Adv Immunol 101: 27-79.

12. Kim S, Poursine-Laurent J, Truscott SM, Lybarger L, Song YJ, et al. (2005) Licensing of natural killer cells by host major histocompatibility complex class I molecules. Nature 436: 709-713.

13. Ruggeri L, Mancusi A, Capanni M, Urbani E, Carotti A, et al. (2007) Donor natural killer cell allorecognition of missing self in haploidentical hematopoietic transplantation for acute myeloid leukemia: challenging its predictive value. Blood 110: 433-440.

14. Kroger N, Shaw B, lacobelli S, Zabelina T, Peggs K, et al. (2005) Comparison between antithymocyte globulin and alemtuzumab and the possible impact of KIR-ligand mismatch after dose-reduced conditioning and unrelated stem cell transplantation in patients with multiple myeloma. $\mathrm{Br} \mathrm{J}$ Haematol 129: 631-643.

15. Shi J, Tricot G, Szmania S, Rosen N, Garg TK, et al. (2008) Infusion of haplo-identical killer immunoglobulin-like receptor ligand mismatched NK cells for relapsed myeloma in the setting of autologous stem cell transplantation. Br J Haematol 143: 641-653.

16. Carbone E, Neri P, Mesuraca M, Fulciniti MT, Otsuki T, et al. (2005) HLA class I, NKG2D, and natural cytotoxicity receptors regulate multiple myeloma cell recognition by natural killer cells. Blood 105: 251-258.

17. El-Sherbiny YM, Meade JL, Holmes TD, McGonagle D, Mackie SL, et al. (2007) The requirement for DNAM-1, NKG2D, and NKp46 in the natural killer cell-mediated killing of myeloma cells. Cancer Res 67: 8444-8449.

18. Sarkar S, Germeraad WT, Rouschop KM, Steeghs EM, van Gelder M, et al. (2013) Hypoxia induced impairment of NK cell cytotoxicity against multiple myeloma can be overcome by IL-2 activation of the NK cells. PLoS One 8: e64835.

19. van Rijn RS, Simonetti ER, Hagenbeek A, Hogenes MC, de Weger RA, et al. (2003) A new xenograft model for graft-versus-host disease by intravenous transfer of human peripheral blood mononuclear cells in RAG2-/- gammac-/- double-mutant mice. Blood 102: 2522-2531.

20. Rozemuller H, van der Spek E, Bogers-Boer LH, Zwart MC, Verweij V, et al. (2008) A bioluminescence imaging based in vivo model for preclinical testing of novel cellular immunotherapy strategies to improve the graft-versus-myeloma effect. Haematologica 93: 1049-1057. 
21. Spanholtz J, Preijers F, Tordoir M, Trilsbeek C, Paardekooper J, et al. (2011) Clinical-grade generation of active NK cells from cord blood hematopoietic progenitor cells for immunotherapy using a closedsystem culture process. PLoS One 6: e20740.

22. Spanholtz J, Tordoir M, Eissens D, Preijers F, van der Meer A, et al. (2010) High log-scale expansion of functional human natural killer cells from umbilical cord blood CD34-positive cells for adoptive cancer immunotherapy. PLoS One 5: e9221.

23. Cany J, van der Waart AB, Tordoir M, Franssen GM, Hangalapura BN, et al. (2013) Natural killer cells generated from cord blood hematopoietic progenitor cells efficiently target bone marrow-residing human leukemia cells in NOD/SCID/IL2Rg(null) mice. PLoS One 8: e64384.

24. Frings PW, Van Elssen CH, Wieten L, Matos C, Hupperets PS, et al. (2011) Elimination of the chemotherapy resistant subpopulation of $4 \mathrm{~T} 1$ mouse breast cancer by haploidentical NK cells cures the vast majority of mice. Breast Cancer Res Treat 130: 773-781.

25. Sutlu T, Alici E (2009) Natural killer cell-based immunotherapy in cancer: current insights and future prospects. J Intern Med 266: 154-181.

26. Alici E, Konstantinidis KV, Sutlu T, Aints A, Gahrton G, et al. (2007) Anti-myeloma activity of endogenous and adoptively transferred activated natural killer cells in experimental multiple myeloma model. Exp Hematol 35: 1839-1846.

27. Garg TK, Szmania SM, Khan JA, Hoering A, Malbrough PA, et al. (2012) Highly activated and expanded natural killer cells for multiple myeloma immunotherapy. Haematologica 97: 1348-1356.

28. Swift BE, Williams BA, Kosaka Y, Wang XH, Medin JA, et al. (2012) Natural killer cell lines preferentially kill clonogenic multiple myeloma cells and decrease myeloma engraftment in a bioluminescent xenograft mouse model. Haematologica 97: 1020-1028.

29. Beider K, Nagler A, Wald O, Franitza S, Dagan-Berger M, et al. (2003) Involvement of CXCR4 and IL-2 in the homing and retention of human NK and NK T cells to the bone marrow and spleen of NOD/SCID mice. Blood 102: 1951-1958.

30. Inngjerdingen M, Damaj B, Maghazachi AA (2001) Expression and regulation of chemokine receptors in human natural killer cells. Blood 97: 367-375.

31. McMillin DW, Delmore J, Negri JM, Vanneman M, Koyama S, et al. (2012) Compartment-Specific Bioluminescence Imaging platform for the high-throughput evaluation of antitumor immune function. Blood 119: e131-138.

32. Van Elssen CH, Vanderlocht J, Oth T, Senden-Gijsbers BL, Germeraad WT, et al. (2011) Inflammationrestraining effects of prostaglandin E2 on natural killer-dendritic cell (NK-DC) interaction are imprinted during DC maturation. Blood 118: 2473-2482.

33. Groen RW, Noort WA, Raymakers RA, Prins HJ, Aalders L, et al. (2012) Reconstructing the human hematopoietic niche in immunodeficient mice: opportunities for studying primary multiple myeloma. Blood 120: e9-e16.

34. Fionda C, Soriani A, Malgarini G, Iannitto ML, Santoni A, et al. (2009) Heat shock protein-90 inhibitors increase $\mathrm{MHC}$ class I-related chain A and B ligand expression on multiple myeloma cells and their ability to trigger NK cell degranulation. J Immunol 183: 4385-4394.

35. Shi J, Tricot GJ, Garg TK, Malaviarachchi PA, Szmania SM, et al. (2008) Bortezomib down-regulates the cell-surface expression of HLA class I and enhances natural killer cell-mediated lysis of myeloma. Blood 111: 1309-1317.

36. Wu X, Shao Y, Tao Y, Ai G, Wei R, et al. Proteasome inhibitor lactacystin augments natural killer cell cytotoxicity of myeloma via downregulation of HLA class I. Biochem Biophys Res Commun 415: 187-192.

37. Bracci L, Moschella F, Sestili P, La Sorsa V, Valentini M, et al. (2007) Cyclophosphamide enhances the antitumor efficacy of adoptively transferred immune cells through the induction of cytokine expression, B-cell and T-cell homeostatic proliferation, and specific tumor infiltration. Clin Cancer Res 13: 644-653.

38. Sharabi A, Ghera NH (2010) Breaking tolerance in a mouse model of multiple myeloma by chemoimmunotherapy. Adv Cancer Res 107: 1-37.

39. Sharabi A, Haran-Ghera N (2011) Immune recovery after cyclophosphamide treatment in multiple myeloma: implication for maintenance immunotherapy. Bone Marrow Res 2011: 269519. 
Chapter 4

40. van Rijn RS, Simonetti ER, Hagenbeek A, Hogenes MC, de Weger RA, (2005) et al. A new xenograft model for graft-versus-host disease by intravenous transfer of human peripheral blood mononuclear cells in RAG2-/- gammac-/- double-mutant mice. Blood 102 :2522-2531. 


\section{Supplemental Table}

Table S4.1 Matched and mismatched KIRs based on genotypic expression of HLA epitopes.

\begin{tabular}{ll}
\hline Cell line & HLA genotype \\
\hline U266 & C1+ C2- Bw4- \\
L363 & C1+ C2- Bw4- \\
LME-1 & C1+C2- Bw4- \\
UM-9 & C1+C2- Bw4- \\
RPMI-8226/s & C1+C2+ Bw4- \\
OPM-1 & C1+C2+ Bw4- \\
XG-1 & C1+C2+ Bw4+ \\
\hline
\end{tabular}

Genotypic expression of HLA-class I epitopes as determined by luminex-SSO. 


\section{Chapter 5}

The synergistic effect of immunomodulatory drugs or monoclonal antibodies on Natural Killer cell mediated anti-Multiple Myeloma responses is lost in an hypoxic environment

Subhashis Sarkar, Wilfred T.V. Germeraad, Evelien E. Boumans, Gerard M.J. Bos, Lotte Wieten In preparation 


\section{Abstract}

Multiple Myeloma ( $\mathrm{MM}$ ) is an incurable disease where the multifaceted bone marrow (BM) environment interaction on the myeloma cells has limited the effective use of Natural Killer (NK) cells. Discovery of new generation chemotherapeutics and monoclonal antibodies (MoAb's) for MM have significantly improved overall survival in the last decade. These novel drugs have demonstrated to display immunomodulatory (IMD) effects on NK cells in vitro or trigger antibody dependent cellular cytotoxicity (ADCC). We therefore hypothesized that combining these drugs with NK cell therapy may provide a successful approach for the treatment of MM. As hypoxia, that is present in the BM environment, has been shown to impair NK cell functionality, and since this factor is often excluded during in vitro testing of IMD drugs or MAob's, the goal of the present study was to investigate the immunomodulatory potential of IMD drugs or MoAb's under hypoxia. To this end, we performed both long-term ( 3 days) and short-term (16 hours) drug treatments on a series of MM cell lines, including U226 and RPMI-8226/S under normoxia (21\% $\mathrm{O}_{2}$ ) or hypoxia ( $1 \%$ or $0.2 \% \mathrm{O}_{2}$ ). Subsequently the IMD treated MM cells were cocultured with IL-2 activated NK cells under the same conditions. We observed that MM cells treated with an array of different IMD anti-MM drugs or MAb's, and cultured under hypoxia $\left(1 \%\right.$ or $\left.0.2 \% \mathrm{O}_{2}\right)$ were not enhancing NK cell cytotoxicity as compared to untreated cells. This study demonstrates the necessity of in vitro anti-myeloma therapeutic research at hypoxic oxygen levels representative of bone marrow environment to be able to draw the right conclusions about new possibilities. 


\section{Introduction}

Multiple Myeloma ( $\mathrm{MM}$ ) is a plasma B cell malignancy which, despite rapid advances in therapy over the last decade, remains an incurable disease ${ }^{1}$. Natural Killer (NK) cell therapy has shown promising results and a first clinical trial for MM has demonstrated safety and feasibility of the approach ${ }^{2}$. However, the complex interplay between bone marrow environment and myeloma cells provides a significant challenge in successful translation of NK cells as a therapeutic regimen for treating MM. We have, in a previous study, demonstrated that one such bone marrow factor, hypoxia can be detrimental for the cytolytic potential of NK cells against $\mathrm{MM}^{3}$. Thus, for successful development of NK cell therapy for $\mathrm{MM}$, better understanding of the myeloma environment, combined with appropriate selection of potent NK cells subsets (Sarkar et al., submitted), cytokine activation and chemotherapeutics are necessary.

NK cells are cells of the innate immune system that can kill a malignant tumor cell without prior sensitization. The recognition of a tumor cell by a NK cell and subsequent activation is regulated by the integrated balance of signaling via activating and inhibitory receptors ${ }^{4}$. The inhibitory receptors (various KIR's and NKG2A) interact with HLA class I molecules on the target cell, while activating receptors (e.g. NKG2D) recognize stress ligands on the tumor cell (MIC's and ULBP's) ${ }^{5}$. An important activating receptor on the NK cell is CD16, which can bind to antibody cross-linked with target cell antigen, and subsequently causes tumor cell lysis by antibody mediated cellular cytotoxicity $(A D C C)^{6}$. The process of NK cell induced cell death involves a complex process of cytoskeletal re-organization to enable successful deposition of cytolytic granules with perforin and granzyme near the tumor NK cell synapse, which subsequently enter tumor cell and activate apoptotic pathways ${ }^{7}$. NK cell induced cellular cytotoxicity is also facilitated by the death ligands on the NK cells, namely TRAIL and FASL, which can directly trigger apoptosis signaling pathways in the tumor, without the requirement of other cytolytic granules. Boosting the potential of these diverse arms of the killing machinery of NK cells can be an effective way to boost NK cell antimyeloma responses. This boost can be achieved by cytokine activation of the NK cells as was shown to occur with IL-12, IL-15 or IL-18 ${ }^{8,9}$, by appropriate selection of chemotherapeutics or immunomodulatory agents or by the combination of NK cells and therapeutic antibodies triggering $A D C C$ all possessing the power to positively modulate NK cell functionality.

Traditional anti-myeloma chemotherapeutics such as melphalan and cyclophosphamide primarily eliminate MM by apoptotic pathways ${ }^{10,11}$. New generation chemotherapeutics for myeloma, such as thalidomide, bortezomib, lenalidomide, and 17-AAG, however eradicate myeloma though novel approaches, such as anti-angiogenesis, proteosome and heat shock protein pathway inhibition ${ }^{12,13}$, and via their influence on the immune system ${ }^{14}$. These new generation chemotherapeutics showed significant improvement in median survival of myeloma patients ${ }^{15-17}$ and some of the clinical studies also observed 
an NK cell boosting effect of the novel compounds. Thalidomide was the first antimyeloma chemotherapeutic suggested to have an immunomodulatory function ${ }^{18}$. In a pioneering study by Faith et al. in 2001, it was shown that myeloma patients treated with $200-800 \mathrm{mg} /$ day thalidomide had a mean 2.6 fold increase in absolute number of NK cells, and additionally patients responding to the treatment had a 3.4 fold increase in total percentage of CD56+ NK cells ${ }^{18}$. More recently, Thalidomide has been replaced by its second generation analogue lenalidomide, a compound also known for its potent immunomodulatory potential ${ }^{19}$. In a recent study, Wolschke et al. demonstrated that low dose lenalidomide can induce strong cell anti-myeloma responses in myeloma patients after allogeneic transplantation, while observing a greater in vitro cytotoxic potential patient derived NK cells ${ }^{20}$.

The clinical observations on the immunomodulatory effect of anti-myeloma agents on NK cells have been complemented with in vitro studies describing the mechanism by which these agents boost NK cell effector functions. Two independent studies demonstrated that in vitro, 5 days treatment of myeloma cells with lenalidomide ${ }^{21}$, and 2 days incubation with $\mathrm{HSP} 90$ inhibitor $17 \mathrm{AAG}^{22}$ resulted in upregulation of NKG2D recognizing ligands, the MIC's and ULBP's, which consequently resulted in greater susceptibility of myeloma cells to NK cell killing ${ }^{21}$. In a different mechanism of action, proteasome inhibitor bortezomib downregulated HLA class I on myeloma cells, and thus subsequently increased their cytotoxicity towards NK cells ${ }^{23}$. These studies clearly demonstrate that combination of new generation immunomodulatory anti-myeloma drugs with NK cell therapy could be a promising approach to treat $\mathrm{MM}^{24,25}$.

An alternative strategy being investigated is the use of monoclonal antibodies for MM that can either directly induce apoptosis in tumor cells, or can mediate effector functions in association with NK cells, by a process called antibody dependent cellular cytotoxicity (ADCC). Thus another approach to modulate NK cell activity in vivo would be to use NK cells in combination with monoclonal antibodies against antigens specifically present on myeloma cell surface ${ }^{26,27}$. Elotuzumab is one such anti-myeloma monoclonal antibody that can bind to the surface CS1 antigen ${ }^{28}$. Daratumumab is another potent monoclonal antibody, which targets the myeloma associated surface antigen $\mathrm{CD} 38^{29}$. More recently, surface $\mathrm{CD} 229$ has been observed to be strongly expressed on myeloma cells, and a monoclonal antibody against CD229 has been show to effectively eliminate myeloma cells in vitro ${ }^{30}$. Nevertheless, the clinical efficacy of these antibodies has not been demonstrated in vivo yet.

Although the new generation immunomodulatory chemotherapeutics and monoclonal antibodies show promising results in vitro and some in delaying myeloma progression in vivo, patients eventually develop resistance to these drugs, suggesting that bone marrow microenvironments provide myeloma cells with immune evasive factors. One such factor is bone marrow hypoxia, which we have previously shown to completely impair immune cell (NK cell) functionality, and this factor is often excluded during in vitro testing of immunomodulatory drugs. Because myeloma cells are mostly present in 
severely hypoxic niches in the bone marrow, as has been shown in several studies and reviewed $^{31,32}$ the goal of the present study was to investigate the influence of hypoxia on the immune-modulatory potential of chemotherapeutic or monoclonal antibodies in eliminating myeloma cells in vitro.

\section{Materials and methods}

\section{Chemotherapeutic drugs and monoclonal antibodies}

lenalidomide was obtained under MTA from Celgene (Summit, NJ, USA), bortezomib was procured as VELCADE (Millennium Pharmaceuticals, Cambridge, MA, USA), 17-AAG was obtained from InvivoGen (SanDiego, CA, USA), and BIIB-021 was purchased from Selleck Chemicals (Houston, TX, USA). Anti-CD229 monocloncal antibody for functional assay was obtained from Santa Cruz Biotechnology (Dallas, TX, USA) ${ }^{30}$.

\section{NK cell isolation and culture}

NK cells were obtained from peripheral blood of healthy donors or buffy coats obtained from the Sanquin blood bank Maastricht, the Netherlands (project 2000-03AZM) or from healthy donors who agreed to donate their blood for research purposes and who had signed an informed consent. PBMC's were obtained by ficoll gradient centrifugation, followed by isolation of CD56+ NK cells with Miltenyi Biotech (Cologne, Germany) NK cell negative selection kit according to manufacturer's instruction. Post isolation, cells were cultured in RPMI-1640 medium supplemented with $10 \%$ FCS, $100 \mathrm{U} / \mathrm{ml}$ penicillin (Gibco) and $100 \mu \mathrm{g} / \mathrm{ml}$ streptomycin (Gibco), and $1000 \mathrm{IU} / \mathrm{ml}$ recombinant human IL-2 (Proleukin, Novartis (Basel, Switzerland)) for 16 hours at 21\% $\mathrm{O}_{2}$. Cells were plated at a density of $4 \times 10^{6}$ cells in $1 \mathrm{ml}$, in 24 -well culture plates. After 16 hours, the activated NK cells were resuspended in their culture plates and directly used for cytotoxicity assays at effector:target (E:T) ratio of 1:1, 2:1, 5:1 or 10:1. In all experiments, NK cells were not treated with any antibody or chemotherapeutic drug during IL-2 activation.

\section{Myeloma cell lines and hypoxic cell culture systems}

In vitro hypoxia experiments were performed using a hypoxia chamber system at $0.2 \%$ $\mathrm{O}_{2}$ or $1 \% \mathrm{O}_{2}$ (Invivo 2,1000 Ruskinn Technology Ltd, Bridgend, UK) at $37^{\circ} \mathrm{C}$ with $5 \% \mathrm{CO}_{2}$. RPMI-8226/S and U266 cells were maintained in RPMI-1640 medium as described previously $^{33}$. RMPI-8226/S cells were plated in 96-well round-bottom plates at a seeding density of 20.000 cells in $150 \mu \mathrm{l}$ medium, and were incubated with bortezomib, 17-AAG, and BIIB-021 for 16 hours at $21 \%$ or $0.2 \% \mathrm{O}_{2}$. As $17-A A G$, and BIIB-021 where dissolved in DMSO, some wells were treated with the same percentage of DMSO as 
control. Each chemotherapeutic drug was tested at two different concentrations; bortezomib: $0.65 \mathrm{nM}$ and $1.30 \mathrm{nM}, \mathrm{BIIB}-021: 0.64 \mu \mathrm{M}$ and $2.55 \mu \mathrm{M}$, and 17-AAG: $0.35 \mu \mathrm{M}$ and $1.40 \mu \mathrm{M}$. Experiments with lenalidomide and anti-CD229 were performed with U266 cells. U266 cells were plated at a concentration of 500.000 cells in $2 \mathrm{ml}$ medium in a 6-well plate. $5 \mu \mathrm{M}$ lenalidomide or control volumes DMSO were added to the culture wells. Cells were cultured for 66 hours at $21 \%$ and $1 \% \mathrm{O}_{2} .160 \mu \mathrm{l}$ of fresh normoxic or hypoxic culture medium was added to the culture well at 24 and 48 hour interval.

\section{Cytotoxicity assay}

Cytotoxic potential of NK cells was determined in a 4-hour flow cytometry based assay as described previously ${ }^{34}$. For experiments with bortezomib, 17-AAG, and BIIB-021, target cells RPMI-8226/S were labeled with DiO (Sigma-Aldrich). After 16 hours of drug treatment of RPMI-8226/S at $0.2 \% \mathrm{O}_{2}$ as described in the previous paragraph, the 96 well plates were sealed with paraflim tape, removed from the hypoxic chambers and centrifuged at $1200 \mathrm{rpm}$ for $9 \mathrm{~min}$. The plates were then put back in the $0.2 \% \mathrm{O}_{2}$ hypoxic chamber, and $100 \mu$ of drug containing media was replaced with $100 \mu$ fresh medium. $100 \mu \mathrm{l}$ of IL-2 activated NK cells at 5:1 E:T ratio was also added to the wells, followed by 4-hours cytotoxicity assay. Each condition was measured in triplicate. For experiments with lenalidomide, 66 hours lenalidomide treated U266 cells at $1 \% \mathrm{O}_{2}$ from all the different conditions were counted and 20.000 U266 cells were plated to 96-well round-bottom plates. This was followed by addition of IL-2 activated NK cells at different E:T ratio's (1:1, 5:1, 10:1) for a 4-hours cytotoxicity assay. To determine ADCC with anti-CD229, $10 \mathrm{\mu g} / \mathrm{ml}$ of the antibody was added to the 66 hours $21 \%$ and $1 \% \mathrm{O}_{2}$ preincubated U266 cells alone or U266 and NK co-cultures (at E:T ratio of 1:1 and 2:1) at the beginning of the assay. For all expermiments the percentage of death cells was determined by flow cytometry after addition of propidium iodide.

\section{Flowcytometry}

U266 cells and NK cells were stained for surface CD229 (clone: 249936, R\&D, State, USA). Briefly, cells were washed, followed by addition of antibody or isotype, and incubated for 30 minutes on ice. Then cells were measured on BD FACS Canto II (BD) and analyzed with FlowJo 7.6.

\section{Statistical analyses}

Influence of hypoxia on NK ADCC was estimated with repeated measures ANOVA with Bonferroni correction. ${ }^{*} P<0.05$ was considered statistically significant. 


\section{Results}

\section{Monoclonal antibody mediated antibody dependent cellular cytotoxicity (ADCC) potential of NK cells against myeloma is lost in a hypoxic \\ environment}

We investigated the ADCC potential of human NK cells against myeloma in an hypoxic environment of $1 \% \mathrm{O}_{2}$. To examine this, we used an anti-CD229 monoclonal that has recently been shown to enhance NK cell cytotoxicity against myeloma cell lines ${ }^{30}$. First, we first analyzed by flowcytometry the expression of CD229 on the myeloma cell line U226, after 66 hours of culture at $21 \%$ or $1 \%$ oxygen and staining with the CD229 antibody. This revealed that U266 was clearly positive for the expression of surface CD229 expression (Figure 5.1A) and that surface expression of CD229 on U226 did not decrease under hypoxia (Figure 5.1B). Human peripheral blood NK cells also had a low expression of this surface antigen (Figure 5.1A). To investigate the influence of hypoxia on NK cell mediated ADCC, we performed an ADCC assay with long-term 66 hours normoxic or hypoxic U266 cells as target cells and IL-2 activated NK cells as effector cells in presence of anti-C229 for 4 hours at $21 \%$ or $1 \% \mathrm{O}_{2}$. The antibody alone was unable to induce cell death of U266 cells, and the percentage of PI positive target cells was comparable to the condition without any antibody (4\% spontaneous cell death; Figure 5.1C). The specific cytotoxicity (as described in materials and methods) of IL-2 activated NK cells against myeloma target cells was $21.1 \%$ in this 4 hours assay at $21 \%$ $\mathrm{O}_{2}$ and $17 \%$ at $1 \% \mathrm{O}_{2}$. In the presence of the anti-CD229 antibody, the activated NK cells could kill $35.5 \%$ of the target myeloma cells which was significantly higher than the specific cytotoxicity measured with NK cells alone at $21 \% \mathrm{O}_{2}$. However, in the ADCC assay under hypoxia, the additive effect of incubation with the anti-CD229 moAb was completely lost, and the specific cytotoxicity was restricted to $19.2 \%$ of target cells, which was comparable to the effect of activated NK cells without CD229 antibody under hypoxia. This phenomenon was consistently observed in $n=4$ donors at an E:T ratio of 1:1 
anti-CD229

A

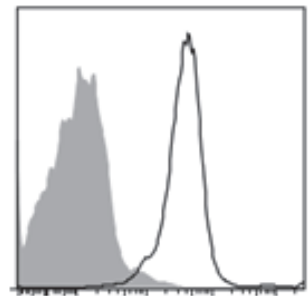

U266

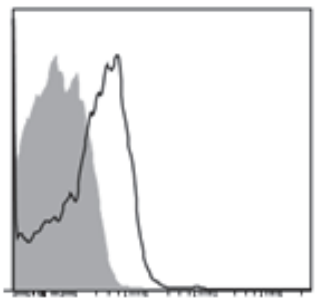

NK cells
B

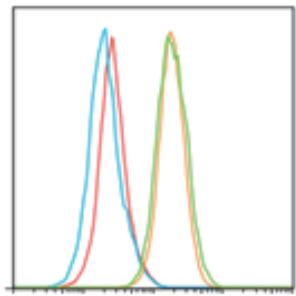

U226 cells

C
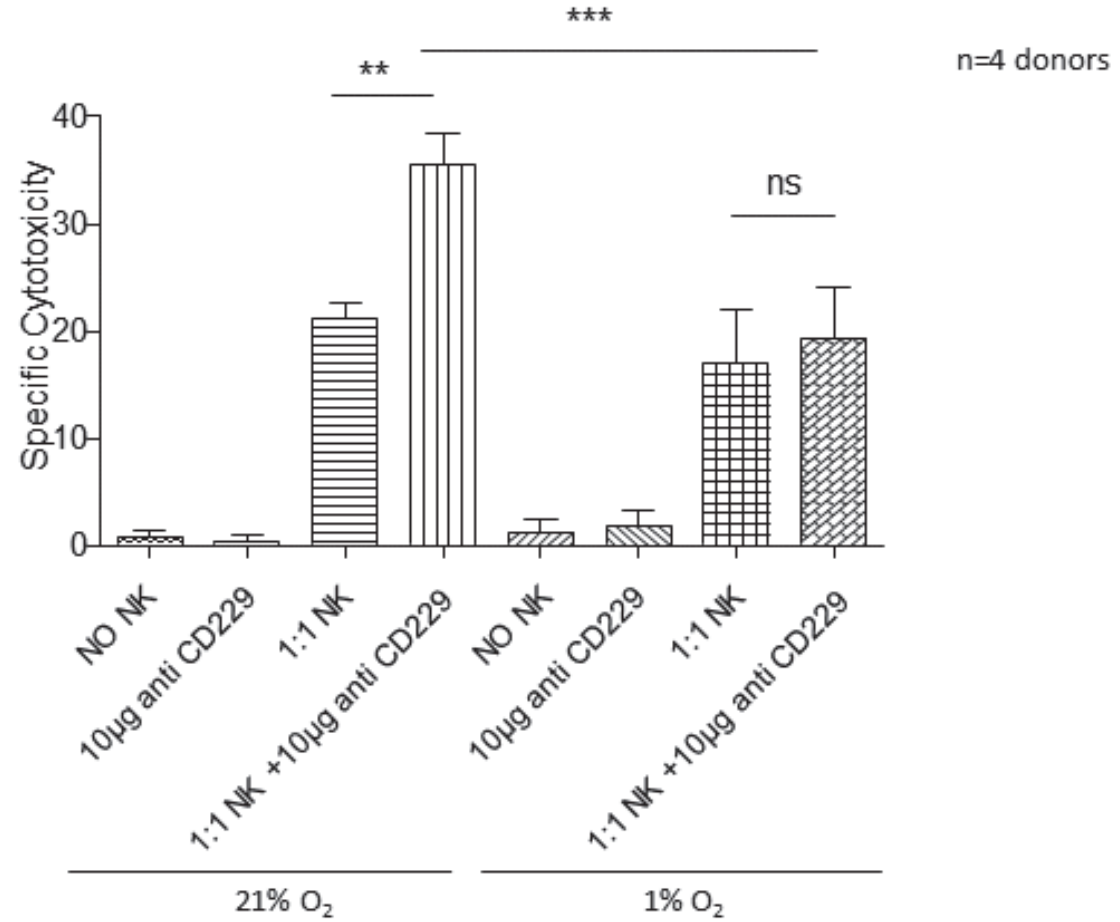

Figure 5.1 Anti CD229 mediated ADCC is impaired under hypoxia.

U266 cells were plated at a concentration of 500.000 cells in $2 \mathrm{ml}$ medium in a 6 well plate. Cells were cultured for 66 hours at $21 \%$ and $1 \% \mathrm{O}_{2} .160 \mu$ l of fresh normoxic or hypoxic culture medium was added to the culture well at 24 and 48 -hour interval. These cells were subsequently used in for flowcytometry and cytotoxicity assays with addition of $1000 \mathrm{IU} / \mathrm{ml} \mathrm{IL}-2 \mathrm{NK}$ cells at 1:1 E:T ratio's for 4 hours \pm anti-CD229, (A) Anti CD229 expression on U266 and NK cells (black lines) is positive as compared to isotype control (grey filled). (B) The expression of CD229 on U226 is not altered under hypoxia $\left(1 \% \mathrm{O}_{2}\right)$ (green histogram) as compared to normoxia $\left(21 \% \mathrm{O}_{2}\right)$ (orange histogram (C) Specific cytoxicity of ADCC triggered by $10 \mu \mathrm{g} / \mathrm{ml} \mathrm{CD229}$ at $21 \% \mathrm{O}_{2}$ or $1 \% \mathrm{O}_{2}$. $\mathrm{Y}$ - axis represents specific cytotoxicity. $X$ - axis represents different combinations of NK cells \pm anti CD229. Bars show mean (+SEM) Statistics were performed with one-way repeated measures ANOVA with Bonferroni correction $* P<0.05,{ }^{* *} P<0.01, * * * P<0.001$. 


\section{Diminished ADCC potential of NK cells against myeloma in hypoxic environment is consistent over a range of antibody concentration and effector NK cell numbers.}

To eliminate the finding that the decreased ADCC of NK cells was a phenomenon being antibody concentration specific or effector cell number specific, we performed the experiments over a range of antibody concentrations $(0-20 \mathrm{ug} / \mathrm{ml}$ of cytotoxicity assay) and with different effector NK cell numbers, using two different NK cell donors. At $21 \%$ $\mathrm{O}_{2}$ - as the antibody concentration of anti-CD229 was increased in the ADCC assay cocultures, the specific cytotoxicity of NK cells against U266 at an 1:1 E:T ratio was also enhanced in an antibody dose dependent manner (Figure 5.2).
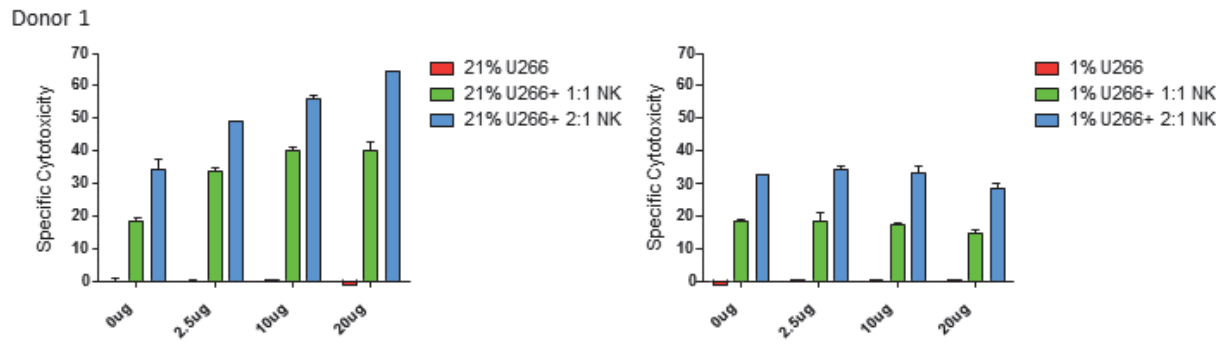

Donor 2
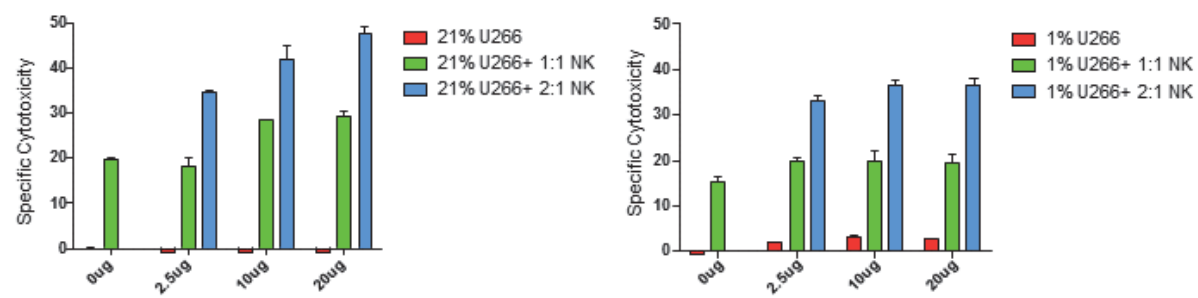

Figure 5.2 Diminished ADCC potential of NK cells against myeloma in hypoxic environment is consistent over a range of antibody concentration and effector NK cell numbers.

U266 cells were plated at a concentration of 500.000 cells in $2 \mathrm{ml}$ medium in a 6 well plate. Cells were cultured for 66 hours at $21 \%$ and $1 \% 02.160 \mu$ of fresh normoxic or hypoxic culture medium was added to the culture well at 24 and 48-hour interval. These cells were subsequently used in cytotoxicity assays with addition of $1000 \mathrm{IU} / \mathrm{ml} \mathrm{IL-2} \mathrm{NK}$ cells at 1:1 and 2:1 $\mathrm{E}: T$ ratio's for 4 hours +/- anti CD229. The Figure shows $\mathrm{n}=2$ donors whose NK cells were tested for ADCC with anti CD229 (0 $\mu \mathrm{g}, 2.5 \mu \mathrm{g}, 10 \mu \mathrm{g}, 20 \mu \mathrm{g}$ ) represented on X -axis. On Y axis is specific cytotoxicity showing mean (+SEM). For each concentration of CD229 antibody, red bars represent U266 with anti CD229 alone. Green bars represent U266 with anti CD229 and 1:1 E:T NK cells. Blue bars represent U266 with anti CD229 and 2:1 E:T NK cells. 
This effect was observed for both donors and the observation was more prominent when the number of effector NK cells was doubled in the cocultures to a 2:1 E:T ratio. For both donors, specific cytotoxicity at $1 \%$ of $\mathrm{O}_{2}$ (18.6\% donor 1 and $15.1 \%$ donor 2 ) was again comparable to specific cytotoxicity at $21 \%$ (18.3\% donor 1 and $19.5 \%$ donor 2). Nevertheless, the additive effect of anti-CD229 was lost under hypoxic levels of $1 \% \mathrm{O}_{2}$, and for all antibody dosages the percentage of specific cytotoxicity was more or less comparable to the level without the anti-CD229 moAb. This demonstrated that even higher doses $(20 \mu \mathrm{g})$ of antibody could not rescue the NK ADCC from the detrimental effect of hypoxia. Doubling the NK cell number, improved overall cytotoxicity against U226 under hypoxia ( $18.6 \%$ at $1: 1$ ratio vs $32.5 \%$ at $1: 2$ ratio). Yet, also at a 2:1 E:T ratio increasing the concentration of antibody did not enhance the cytotoxicity of NK cells. This observation suggests that the immunomodulatory effect of monoclonal antibodies, at least of the anti-CD229 moAb, on NK cells is lost under hypoxia. Further investigation is needed to investigate whether this is also true for other new generation anti-myeloma monoclonal antibodies and chemotherapeutics.

\section{Treatment of myeloma cells with immunomodulatory drugs fails to induce a synergistic effect with IL-2 activated NK cells at $1 \%$ of oxygen}

In this study, we also investigated the potential of several new generation antimyeloma compounds that have previously been proposed to have NK cell boosting activity, in an hypoxic environment. To this end, we used a flow cytometry based assay to study cytoxicity of IL-2 activated NK cells from $n=4$ donors against U266 MM target cells that were incubated for 66 hours at $1 \% \mathrm{O}_{2}$ and with or without $5 \mu \mathrm{M}$ lenalidomide. Treatment of $\mathrm{U} 266$ myeloma cell line at $1 \% \mathrm{O}_{2}$ for 66 hours with $5 \mu \mathrm{M}$ lenalidomide did not induce any significant cellular death. Cell death was determined by propidium iodide (PI) staining using flowcytometry and percentages of PI positive U266 cells were comparable for both conditions. Addition of IL-2 activated NK cells to hypoxic myeloma cells induced cytotoxic responses of approximately 20\% (E:T 1:1), 50\% (E:T 5:1), 70\% (E:T 10:1), respectively (Figure 5.3). The addition of IL-2 activated NK cells to lenalidomide treated hypoxic myeloma cells resulted in cytotoxicity percentages that were more or less comparable to the percentages detected with target cells that were not treated with lenalidomide. This was true at all tested E:T ratio's and demonstrated that 66 hours incubation with lenalidomide failed to improve NK cell cytotoxicity against U266 at $1 \%$ O. Furthermore, for some NK cell donors, we observed that the cytotoxicity was even suppressed for lenalidomide treated hypoxic cells. 


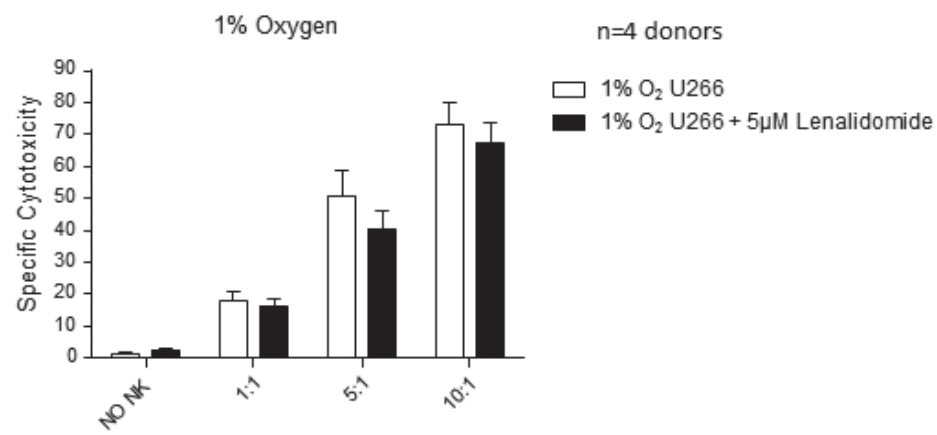

Figure 5.3 Lack of additive effect of lenalidomide and NK cells at $1 \% \mathrm{O}_{2}$ :

U266 cells were plated at a concentration of 500.000 cells in $2 \mathrm{ml}$ medium in a 6 well plate. $5 \mu \mathrm{M}$ lenalidomide or control volumes DMSO was added to the culture wells. Cells were cultured for 66 hours at $1 \% \mathrm{O}_{2} .160 \mu \mathrm{l}$ of fresh normoxic or hypoxic culture medium was added to the culture well at 24 and 48 hour interval. Lenalidomide treated (black bars) or control PBS (white bars) treated U266 were subsequently combined with $1000 \mathrm{IU} / \mathrm{ml}$ IL-2 activated NK cells in different E:T ratio (NO NK, 1:1, 5:1, 10:1) as represented on X-axis. Y-axis represents specific cytotoxicty showing mean (+SEM).

Next, we determined the immunomodulatory potential of water solved bortezomib, and DMSO solved BIIB-021 and 17-AAG. To this end, the myeloma cell line RPMI-8226/S was incubated for 16 hours with the different drugs at $0.2 \% \mathrm{O}_{2}$ followed by analysis of NK cell cytotoxicity against drug incubated target cells in a 4 hours cytotoxicity assay at $0.2 \% \mathrm{O}_{2}$. In these assays, the drug was washed out with fresh medium before addition of the NK cells. In all experiments, IL-2 activated NK cells derived from buffy coat donors were used as effector cells. For each compound, the immunomodulatory potential was examined at two different drug concentrations. For proteosome inhibitor bortezomib it was $0.65 \mathrm{nM}$ and $1.30 \mathrm{nM}$, the HSP90 inhibitor's BIIB-021, was tested at $0.64 \mu \mathrm{M}$ and $2.55 \mu \mathrm{M}$, and 17-AAG at $0.35 \mu \mathrm{M}$ and $1.40 \mu \mathrm{M}$. Cell death in the control condition (incubation at $0.2 \% \mathrm{O}_{2}$ in the absence of bortezomib) and in the condition incubated with $0.65 \mathrm{nM}$ bortezomib was low (7\%; Figure 5.4A). The percentage of death target cells was higher upon incubation with $1.30 \mathrm{nM}$ bortezomib (18.5\%). In this 4-hour assay, NK cells killed $44 \%$ of untreated target cells. The percentage of death target cells in the conditions with bortezomib pre-incubation and NK cells was comparable to the percentage of killed target cells with NK cells alone showing that bortezomib pre-incubation, under these conditions, did not enhance susceptibility of the target cells for killing by NK cells. Because BIIB-021 and 17-AAG where solved in DMSO, we also evaluated as a control the effect of DMSO pretreatment on RPMI$8226 / s$ viability and NK cell killing. This revealed that DMSO pre-incubation by itself did not trigger MM cell death and did not have an effect on NK cell killing (Figure 5.4B upper panel). 
Bortezomib $0.2 \% \mathrm{O}_{2}$

A

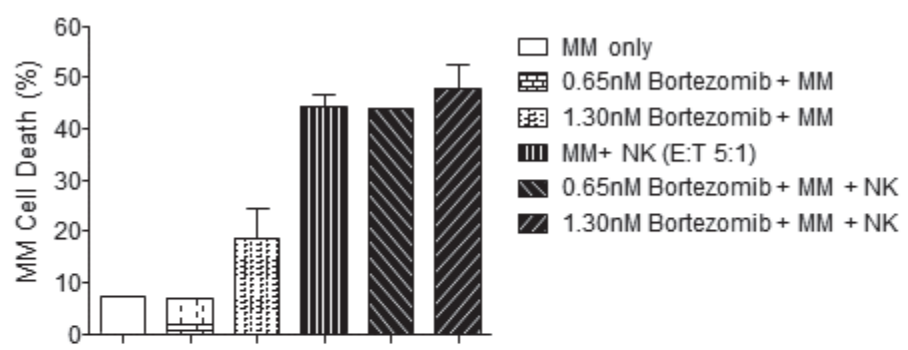

B
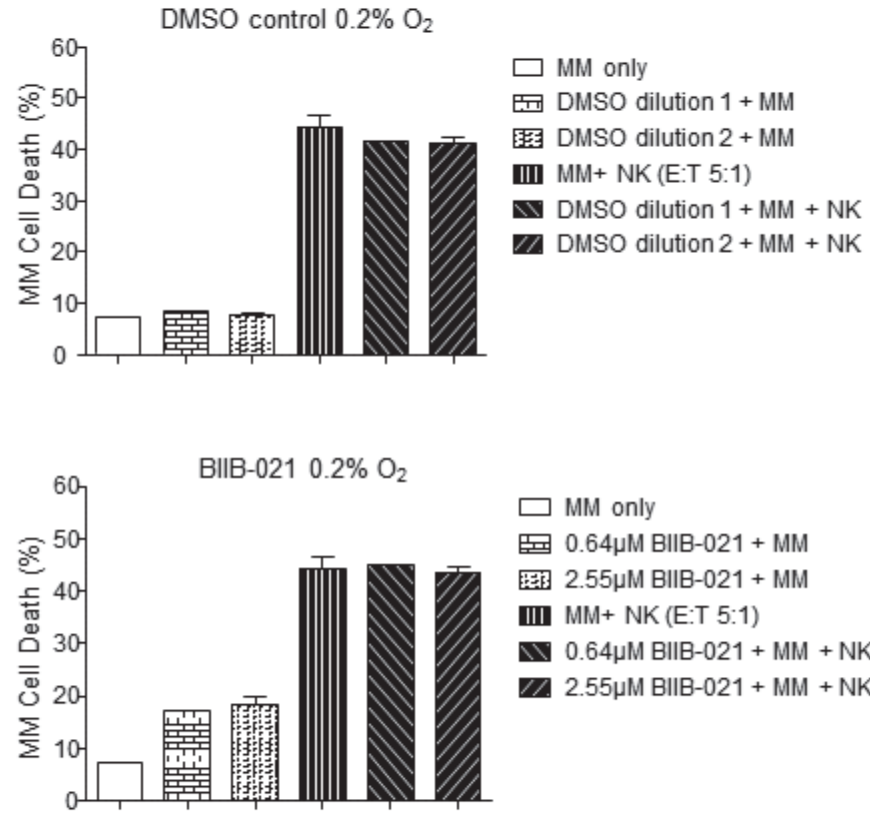

$\square$ MM only

臣 $0.64 \mu \mathrm{M}$ BIIB- $021+$ MM

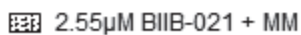

III $M M+N K(E: T$ : $: 1)$

IV $0.64 \mu \mathrm{M}$ BIIB- $021+\mathrm{MM}+\mathrm{NK}$

V] $2.55 \mu \mathrm{M}$ BIIB-021 + MM + NK
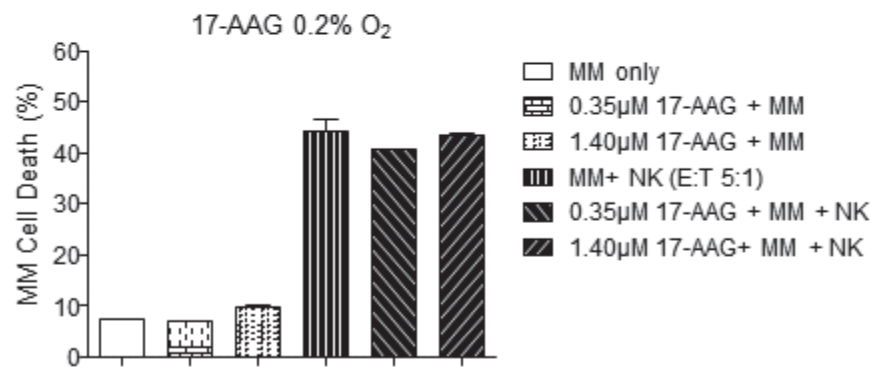

MM = RPMI-8226/S 
Figure 5.4 Treatment of RPMI-8226/S with proteasome inhibitor or HSP90 inhibitor drugs does not induce synergistic effect with IL-2 activated NK cells at $1 \%$ of oxygen

Dio labelled RPMI-8226/S were treated with bortezomib,17-AAG, and BIIB-021. After 16 hours of drug treatment of RPMI-8226/S at $0.2 \% \mathrm{O}_{2}$, the drugs were washed out followed by addition of IL-2 activated NK cells at 5:1 E:T ratio for 4 hours (A)Water soluble bortezomib and (B) DMSO soluble immunomodulatory anti-myeloma drug 17-AAG and BIIB-021 were tested for their immunomodulatory activity at $0.2 \% \mathrm{O}_{2}$. Total $\mathrm{MM}$ cells death was determined by propidium iodide (Y-axis).

Pre-incubation with BIIB-021, at both concentrations, enhanced the percentage of death $\mathrm{MM}$ cells $(7.4 \%$ in the control vs $17.2 \%$ and $18.4 \%$ in presence of $0.64 \mu \mathrm{M}$ and $2.55 \mu \mathrm{M}$.BIIB-021; Figure 5.4B middle panel). However, it did not have an influence on the percentage of death target cells in the conditions with NK cells. 17-AAG preincubation by itself did not induce target cell death nor did it enhance the killing of target cells by the NK cells (Figure 5.4B lower panel). Thus, in contrast to literature showing an effect at $21 \% \mathrm{O} 2$, we did neither observe an additive nor a synergistic effect of the immunomodulatory drugs with NK cells at $0.2 \% \mathrm{O} 2$ These observations suggest a lack of synergy between immunomodulatory drugs and NK cells in different hypoxic conditions, emphasizing the importance of performing in vitro anti-myeloma therapeutic research at hypoxic oxygen levels representative of bone marrow environment.

\section{Discussion}

Conventional monoclonal antibody therapy for myeloma has mostly focused on blocking the key pro-survival signaling molecules between bone marrow stromal cells and myeloma cells, such as IL-6 and VEGF. More recently, with the identification of new myeloma specific tumor antigens, such as $C D 229^{30}$, and generation of high avidity antibodies (Daratumumab) for conventional myeloma cell surface antigen $\mathrm{CD}^{2} 8^{29}$, interest has shifted to harness the immune boosting potential of anti-myeloma monoclonal antibodies. Monoclonal antibodies can bind cell surface antigen on myeloma cell through the Fab region while simultaneously binding to the FCyRIII receptor (CD16) on the NK cell via the Fc fragment ${ }^{35}$. This interaction of the monoclonal antibody between the target cell and NK cell will activate the NK cell, which can subsequently induce antibody dependent cellular cytotoxicity (ADCC). Other monoclonal antibodies such as Trastuzumab (Herceptin ${ }^{\circledR}$ ) can block dimerization of HER2 receptors on tumor cells and subsequently block proliferative signaling pathways in addition to activating ADCC mediated NK cell lysis ${ }^{36}$.

In the present study, we observed that IL-2 activated NK cell mediated ADCC against MHC class I positive U266 cell line was completely abrogated under hypoxia $\left(1 \% \mathrm{O}_{2}\right)$. We show this phenomenon for the monoclonal antibody targeting myeloma cell 
surface antigen CD229, which has been shown to mediate potent ADCC mediated cell lysis of U266 under normoxia ${ }^{30}$. Because hypoxic and normoxic cells showed the same level of CD229 cell surface expression (Figure 5.1), it seems unlikely that limited availability of the CD229 antigen caused the decrease in cytotoxicity under hypoxia. In a previous study ${ }^{34}$, we observed a minor decrease in CD16 expression by the NK cells upon 16 hours of culture at $0 \% \mathrm{O}_{2}$. This minor reduction in CD16 expression was confirmed by another study, although there the authors also showed that the triggering capacity of CD16 was not influenced by hypoxia ${ }^{37}$. Recently, a detrimental effect of hypoxia on NK cell mediated killing of breast cancer cells was attributed to hypoxia mediated resistance acquired by tumor cells, as hypoxia induced autophagy resulted in enhanced degradation of granzymes by the tumor cells and consequently a lower level of tumor cell killing ${ }^{38}$. For MM, we demonstrated that the detrimental effect of hypoxia on NK cell cytotoxicity could be overcome by IL-2 activation of the NK cell and we showed that NK cell degranulation was not influenced by hypoxia ${ }^{34}$. The NK cells in the present study were IL-2 activated and in line with our previous study, we did not observe a significant difference in NK cell cytotoxicity at $21 \%$ and $1 \% \mathrm{O}_{2}$. The additive effect of CD229 triggered ADCC was, however, lost under hypoxia and it would be interesting to investigate whether killing pathways other than the release of cytotoxic granules, such as TRAIL and FASL induced cell death are involved in ADCC killing and what the influence of hypoxia on these pathways is.

In the current study, when we tested lenalidomide for its NK cell boosting effect, at a concentration frequently described in literature $\left(5 \mu \mathrm{M}^{21}\right)$, we found that hypoxia cultured myeloma cells treated with lenalidomide were no more susceptible to NK cell cytotoxicity as compared to untreated cells. This phenomenon was also observed under hypoxia for other immunomodulatory drugs as bortezomib, 17-AAG, and BIIB-021, wherein these drugs failed to sensitize drug treated myeloma cells for better NK cell killing. Previous in vitro studies have demonstrated that immunomodulatory drugs like bortezomib, lenalidomide, and 17-AAG can have a direct NK cell boosting effect using in vitro assay's performed at $21 \% \mathrm{O}_{2}{ }^{21-23}$ advocating combination therapy of these drugs with NK cell therapy. However, these studies have not taken into consideration the influence of hypoxia on NK cell functionality and in our system when we test these same drugs for NK cell boosting effect under hypoxia, we do not observe any enhancing effect as has been described in literature under normoxia. This raises a very critical issue in that testing immunomodulatory drugs should be performed under oxygen concentrations that are relevant for the bone marrow, thus low oxygen. Atmospheric oxygen concentrations are obsolete and non-informative.

New generation anti-myeloma chemotherapeutics have been demonstrated to increase NK cell numbers in peripheral blood of $\mathrm{MM}$ patients ${ }^{18}$ and a possible immunomodulation of NK cells have been demonstrated in in vitro experiments ${ }^{39}$. This effect is mostly the result of a cross talk between different immune cells post chemotherapeutic treatment. Hsu et al. ${ }^{40}$ showed that in vitro lenalidomide stimulates CD4+ T cells to 
produce more IL-2, which can subsequently enhance NK cell cytotoxicity. This observation consolidated the earlier study by Hayashi et al. $^{39}$, which demonstrated that thalidomide and other immunomodulatory anti-myeloma drugs facilitate the nuclear translocation of NFAT-2 in T cells, thereby increasing IL-2 transcription in T cells. In in vivo models, lenalidomide was found to stimulate dendritic cells, which could subsequently activate NK cells in a Raji-bearing SCID mice model ${ }^{41}$. This immune cell cross talk could explain the recent success of immunomodulatory anti-myeloma drugs in the clinical setting. These in vivo data suggest that immunomodulatory drugs such as bortezomib or lenalidomide may still exert innate immune cell activation and boosting in vivo via other cell - cell interactions, and that whether immunomodulatory drugs have a direct NK cell activating properties capacity to sensitize MM cells for NK cell killing needs careful investigation in the hypoxic environment representative of bone marrow in myeloma.

Our in vitro observations in a hypoxic environment do not discard the notion that immunomodulatory drugs can have potent anti-myeloma response in patients, mostly due to a boost in immune cell crosstalk and cytokine production. However, using these immunomodulatory drugs in combination with NK cell therapy might not significantly improve anti-myeloma responses triggered by enhancing NK cell effector functions, as these compounds do not sensitize myeloma cells to enhanced NK mediated killing in a hypoxic environment. For combination with NK cell therapy, anti-myeloma immunomodulatory drugs should be able to eliminate myeloma cells in a hypoxic environment. Thus, we recommend drug screenings under hypoxia and presumably other bone marrow microenvironmental factors as well such as stromal cells and cytokines like IL-6, among others, which are important for MM survival in vivo ${ }^{42,43}$.

\section{Acknowledgements}

S. Sarkar was supported by a PhD grant from GROW, School of Oncology and Developmental Biology. L. Wieten was supported by a personal grant from Dutch Cancer association (KWF kankerbestrijding; UM2012-5375). G. Bos and L. Wieten were supported by a grant from the Kankeronderzoeksfonds Limburg. 


\section{REFERENCES}

1. Eshaghian S, Berenson JR (2012) Multiple myeloma: improved outcomes with new therapeuticapproaches. Curr Opin Support Palliat Care 6: 330-336.

2. Shi J, Tricot G, Szmania S, Rosen N, Garg TK, et al. (2008) Infusion of haplo-identical killer immunoglobulin-like receptor ligand mismatched NK cells for relapsed myeloma in the setting of autologous stem cell transplantation. Br J Haematol 143: 641-653.

3. Ali N, Paul S, Gayen D, Sarkar SN, Datta K, et al. (2013) Development of low phytate rice by RNAi mediated seed-specific silencing of inositol 1,3,4,5,6-pentakisphosphate 2-kinase gene (IPK1). PLoS One 8: e68161.

4. Vivier E, Raulet DH, Moretta A, Caligiuri MA, Zitvogel L, et al. (2011) Innate or adaptive immunity? The example of natural killer cells. Science 331: 44-49.

5. Carbone E, Neri P, Mesuraca M, Fulciniti MT, Otsuki T, et al. (2005) HLA class I, NKG2D, and natural cytotoxicity receptors regulate multiple myeloma cell recognition by natural killer cells. Blood 105: 251-258.

6. Smyth MJ, Cretney E, Kelly JM, Westwood JA, Street SE, et al. (2005) Activation of NK cell cytotoxicity. Mol Immunol 42: 501-510.

7. Bryceson YT, Chiang SC, Darmanin S, Fauriat C, Schlums H, et al. (2011) Molecular mechanisms of natural killer cell activation. J Innate Immun 3: 216-226.

8. Ni J, Miller M, Stojanovic A, Garbi N, Cerwenka A (2012) Sustained effector function of IL-12/15/18preactivated NK cells against established tumors. J Exp Med 209: 2351-2365.

9. Romee R, Schneider SE, Leong JW, Chase JM, Keppel CR, et al. (2012) Cytokine activation induces human memory-like NK cells. Blood 120: 4751-4760.

10. Gomez-Bougie P, Oliver L, Le Gouill S, Bataille R, Amiot M (2005) Melphalan-induced apoptosis in multiple myeloma cells is associated with a cleavage of $\mathrm{Mcl}-1$ and $\mathrm{Bim}$ and a decrease in the Mcl-1/Bim complex. Oncogene 24: 8076-8079.

11. Minarik J, Scudla V, Ordeltova M, Bacovsky J, Pika T, et al. (2009) Prognostic significance of apoptotic index in multiple myeloma patients treated by conventional therapy and novel agents, thalidomide and bortezomib. Eur J Haematol 83: 528-534.

12. Usmani SZ, Chiosis G (2011) HSP90 inhibitors as therapy for multiple myeloma. Clin Lymphoma Myeloma Leuk 11 Suppl 1: S77-81.

13. Mujtaba T, Dou QP (2011) Advances in the understanding of mechanisms and therapeutic use of bortezomib. Discov Med 12: 471-480.

14. Quach H, Ritchie D, Stewart AK, Neeson P, Harrison S, et al. (2010) Mechanism of action of immunomodulatory drugs (IMiDS) in multiple myeloma. Leukemia 24: 22-32.

15. van de Donk NW, Lokhorst HM (2013) New developments in the management and treatment of newly diagnosed and relapsed/refractory multiple myeloma patients. Expert Opin Pharmacother 14: 1569-1573.

16. Orlowski RZ (2013) Novel agents for multiple myeloma to overcome resistance in phase III clinical trials. Semin Oncol 40: 634-651.

17. Lee HC, Shah JJ, Orlowski RZ (2013) Novel approaches to treatment of double-refractory multiple myeloma. Am Soc Clin Oncol Educ Book.

18. Davies FE, Raje N, Hideshima T, Lentzsch S, Young G, et al. (2001) Thalidomide and immunomodulatory derivatives augment natural killer cell cytotoxicity in multiple myeloma. Blood 98: 210-216.

19. Dauguet N, Fournie JJ, Poupot R, Poupot M (2010) Lenalidomide down regulates the production of interferon-gamma and the expression of inhibitory cytotoxic receptors of human Natural Killer cells. Cell Immunol 264: 163-170.

20. Wolschke C, Stubig T, Hegenbart U, Schonland S, Heinzelmann M, et al. (2013) Postallograft lenalidomide induces strong NK cell-mediated antimyeloma activity and risk for T cell-mediated GvHD: Results from a phase I/II dose-finding study. Exp Hematol 41: 134-142 e133.

21. Benson DM, Jr., Bakan CE, Zhang S, Collins SM, Liang J, et al. (2011) IPH2101, a novel anti-inhibitory KIR antibody, and lenalidomide combine to enhance the natural killer cell versus multiple myeloma effect. Blood 118: 6387-6391. 
22. Fionda C, Soriani A, Malgarini G, lannitto ML, Santoni A, et al. (2009) Heat shock protein-90 inhibitors increase $\mathrm{MHC}$ class I-related chain A and B ligand expression on multiple myeloma cells and their ability to trigger NK cell degranulation. J Immunol 183: 4385-4394.

23. Shi J, Tricot GJ, Garg TK, Malaviarachchi PA, Szmania SM, et al. (2008) Bortezomib down-regulates the cell-surface expression of HLA class I and enhances natural killer cell-mediated lysis of myeloma. Blood 111: 1309-1317.

24. Krieg S, Ullrich E (2012) Novel immune modulators used in hematology: impact on NK cells. Front Immunol 3: 388.

25. De Raeve H, Vanderkerken K (2006) Immunomodulatory drugs as a therapy for multiple myeloma. Curr Pharm Biotechnol 7: 415-421.

26. Allegra A, Penna G, Alonci A, Russo S, Greve B, et al. (2013) Monoclonal antibodies: potential new therapeutic treatment against multiple myeloma. Eur J Haematol 90: 441-468.

27. Tai YT, Anderson KC (2011) Antibody-based therapies in multiple myeloma. Bone Marrow Res 2011: 924058.

28. Hsi ED, Steinle R, Balasa B, Szmania S, Draksharapu A, et al. (2008) CS1, a potential new therapeutic antibody target for the treatment of multiple myeloma. Clin Cancer Res 14: 2775-2784.

29. de Weers M, Tai YT, van der Veer MS, Bakker JM, Vink T, et al. (2011) Daratumumab, a novel therapeutic human CD38 monoclonal antibody, induces killing of multiple myeloma and other hematological tumors. J Immunol 186: 1840-1848.

30. Atanackovic D, Panse J, Hildebrandt Y, Jadczak A, Kobold S, et al. (2011) Surface molecule CD229 as a novel target for the diagnosis and treatment of multiple myeloma. Haematologica 96: 1512-1520.

31. Hu J, Van Valckenborgh E, Menu E, De Bruyne E, Vanderkerken K (2012) Understanding the hypoxic niche of multiple myeloma: therapeutic implications and contributions of mouse models. Dis Model Mech 5: 763-771.

32. Colla S, Storti P, Donofrio G, Todoerti K, Bolzoni M, et al. (2010) Low bone marrow oxygen tension and hypoxia-inducible factor-1alpha overexpression characterize patients with multiple myeloma: role on the transcriptional and proangiogenic profiles of CD138(+) cells. Leukemia 24: 1967-1970.

33. Rozemuller H, van der Spek E, Bogers-Boer LH, Zwart MC, Verweij V, et al. (2008) A bioluminescence imaging based in vivo model for preclinical testing of novel cellular immunotherapy strategies to improve the graft-versus-myeloma effect. Haematologica 93: 1049-1057.

34. Sarkar S, Germeraad WT, Rouschop KM, Steeghs EM, van Gelder M, et al. (2013) Hypoxia induced impairment of NK cell cytotoxicity against multiple myeloma can be overcome by IL-2 activation of the NK cells. PLoS One 8: e64835.

35. Mellor JD, Brown MP, Irving HR, Zalcberg JR, Dobrovic A (2013) A critical review of the role of Fc gamma receptor polymorphisms in the response to monoclonal antibodies in cancer. J Hematol Oncol 6: 1.

36. Nahta R, Esteva FJ (2006) Herceptin: mechanisms of action and resistance. Cancer Lett 232: 123-138.

37. Balsamo M, Manzini C, Pietra G, Raggi F, Blengio F, et al. (2013) Hypoxia downregulates the expression of activating receptors involved in NK-cell-mediated target cell killing without affecting ADCC. Eur J Immunol 43: 2756-2764.

38. Baginska J, Viry E, Berchem G, Poli A, Noman MZ, et al. (2013) Granzyme B degradation by autophagy decreases tumor cell susceptibility to natural killer-mediated lysis under hypoxia. Proc Natl Acad Sci U S A 110: 17450-17455.

39. Hayashi T, Hideshima T, Akiyama M, Podar K, Yasui H, et al. (2005) Molecular mechanisms whereby immunomodulatory drugs activate natural killer cells: clinical application. Br J Haematol 128: 192-203.

40. Hsu AK, Quach H, Tai T, Prince HM, Harrison SJ, et al. (2011) The immunostimulatory effect of lenalidomide on NK-cell function is profoundly inhibited by concurrent dexamethasone therapy. Blood 117: 1605-1613.

41. Reddy N, Hernandez-Ilizaliturri FJ, Deeb G, Roth M, Vaughn M, et al. (2008) Immunomodulatory drugs stimulate natural killer-cell function, alter cytokine production by dendritic cells, and inhibit angiogenesis enhancing the anti-tumour activity of rituximab in vivo. Br J Haematol 140: 36-45.

42. McMillin DW, Delmore J, Negri JM, Vanneman M, Koyama S, et al. (2012) Compartment-Specific Bioluminescence Imaging platform for the high-throughput evaluation of antitumor immune function. Blood 119: e131-138. 
Chapter 5

43. Zhang XG, Klein B, Bataille R (1989) Interleukin-6 is a potent myeloma-cell growth factor in patients with aggressive multiple myeloma. Blood 74: 11-13. 


\section{Chapter 6}

General discussion 
Chapter 6 


\section{General discussion}

Multiple Myeloma (MM) is a haematological malignancy that remains incurable. For more than 50 years, cytotoxic regimens and auto-HSCT have been the mainstay therapeutic approaches. Since 2000, a major step forward towards treating MM has been achieved with the introduction of new drugs like lenalidomide and bortezomib ${ }^{1,2}$. However, even with these new generation of anti-myeloma agents, a fraction of the MM cells remains drug resistant, ultimately resulting in disease relapse and MM progression $^{3}$. Thus intense research is currently underway to investigate approaches that utilize other methods like antibodies or immune cells to eliminate MM cells in general, and more specifically therapy resistant cells. This thesis described such an approach.

The application of allogeneic stem cell transplantation has previously provided longterm cure in a small group of patients. However, allogeneic stem cell transplantation is not a standard choice of therapy in MM due to very high incidence of treatment related mortality, progression of disease in most of the patients and sometimes the lack of a suitable matching donor. Haploidentical stem cell transplantation, where the donor shares half of the haplotype of the patient overcomes the limitation of donor availability. Ruggeri et al. demonstrated in 2002 that haploidentical stem cell transplantation contains a graft versus leukemia (AML) effect, which was shown to be mediated by Natural Killer (NK) cells ${ }^{4}$, without a graft versus host (GvH) effect. An observation that is in line with the option that NK cells might be relevant also for MM has been made by Kroger et al., where they demonstrated that after donor transplantation making use of an unrelated donor, patients with a KIR-ligand mismatch had a better relapse free survival than the patients that did not have such a KIR-ligand mismatch allowing NK cell alloactivity ${ }^{5}$.

Our group has demonstrated the tumor elimination potential of haploidentical stem cell transplantation together with nonmyeloablative conditioning in a breast cancer mice model ${ }^{6}$. Furthermore, our group recognized NK cells as the key immune cells eliminating the tumor cells in this breast cancer model ${ }^{7}$.

As $\mathrm{MM}$ remains an incurable disease with a median survival of 5-6 years, there is an urgent need to find novel therapies to cure this disease. MM has been shown to be sensitive to immune cells following donor lymphocyte infusion (DLI) ${ }^{8}$. However, most patients suffer from GVHD, which is therefore a major limitation of $\mathrm{DLI}^{9}$. As one of the components of DLI are the NK cells, and having observed anti-tumor effect of mouse NK cells in eliminating mouse breast cancer, we hypothesized that by identifying bone marrow factors that impair human NK cell activity, and methods to overcome such inhibitory factors, NK cells could become a therapeutic reality in human. Such inhibitory 
factors can be provided either at the tumor microenvironmental level or cellular level, with the ultimate result of tumor survival. Blocking and/or activating these immunoediting factors could pave the way for successful therapeutic application of NK cell therapy against MM.

\section{This thesis described a four step integrated approach in identifying factors which impair NK cell activity in vivo and how we can overcome these inhibitory factors towards the ultimate goal NK cell therapy for $\mathrm{MM}$}

Our ultimate goal is to develop NK cell therapy that can cure patients with MM. In our study, we first aimed to investigate one of the most important hallmarks of cancer, its microenvironmental milieu, rich in factors with accessory cells, suppressor cells, and a large number of known and unknown immunosuppressive factors ${ }^{10}$. These microenvironmental inhibitory factors - like hypoxia - form the basis of tumor progression, as NK and T cell surveillance is affected, leading to subsequent escape of proliferating tumor cells (Chapter 2). The next step in the process of developing NK cell therapy was to identify the most potent allogeneic effector cells in combination with appropriate cytokine stimulation that can mount substantial anti-MM responses (Chapter 3). After eliminating the microenvironmental and cellular inhibitory factors, which provide protection to MM cells against NK cells, we next aimed to find a curative regimen in an animal model for NK cell therapy in combination with existing chemoand radiotherapy (Chapter 4). In the last part of this process development, we returned to in vitro approaches representative of the bone marrow microenvironment, to further boost NK cell therapy, by sensitizing MM with immunomodulatory drugs or antibodies, and enhancing their susceptibility to NK cell killing (Chapter 5).

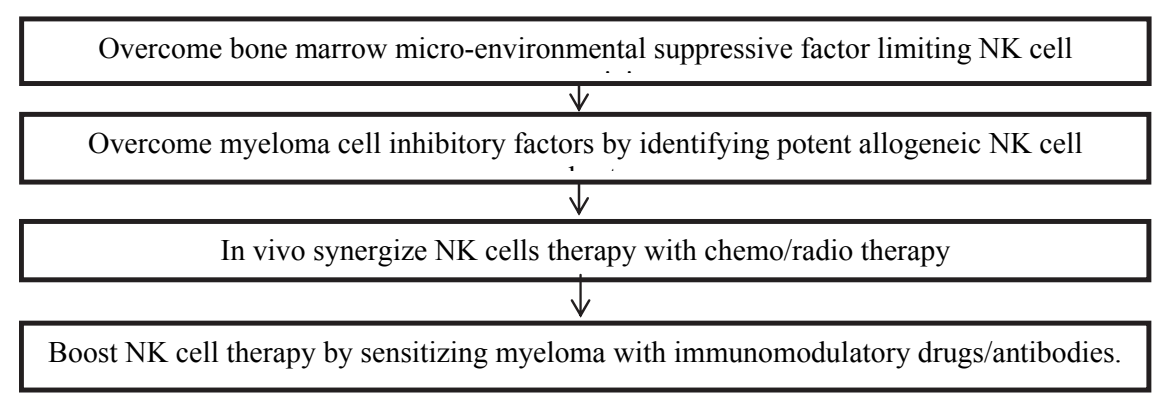

Figure 6.1 A four step integrated approach in identifying factors that impair NK cell activity in vivo and how we can overcome these inhibitory factors towards the ultimate goal NK cell therapy for MM 


\section{MM disease progression and NK cell evasion}

NK cells are important immune cells that control tumor progression at an early stage. This is because at early stages, the tumor downregulates HLA class I, which helps it to evade the T cells. However, studies also show that as the disease progresses in patients, MM cells have the capacity to adapt at cellular level to evade NK cell lysis by increasing expression of inhibitory molecules such as HLA class $1^{11}$. At the microenvironmental level, MM outgrowth is further assisted by impairing the NK cell directly; NK cells derived from $\mathrm{MM}$ patients were shown to have down-regulated the key activating receptors 2B4/CD244 and CD16 ${ }^{12}$. Furthermore, Yasser et al. demonstrated significantly lower expression of the activating receptor DNAM-1 in NK cells from MM patients ${ }^{13}$. Another key feature of the disease is excess production of IgG light chains (also called M-protein) by the malignant plasma cells. This tumor-derived factor was shown to severely dampen NK cell effector functions ${ }^{14}$. These studies demonstrate that both at microenvironmental and cellular level, MM cells manage to evade autologous NK cell surveillance, which over time leads to disease progression and death.

\section{Tailoring NK cells to kill MM cells in a hypoxic bone marrow microenvironment}

MM cells reside and disseminate in a bone marrow environment with a widespread distribution of hypoxic niches. This microenvironmental characteristic has been overlooked while determining the pre-clinical efficacy of novel anti-MM therapeutics, and more specifically immunotherapy. Moreover, continuous growth of tumor cells, as in solid tumors, can also lead to extensive regions of tumor associated hypoxia and has been shown to protect those parts of the tumor from chemotherapy, and can be mechanistically explained by activation of STAT-3 or Major Vault Protein (MVP) overexpression $^{15,16}$. Benito et al. demonstrated that the bone marrow in acute lymphoblastic leukemia (ALL) is extensively hypoxic, and hypoxia promotes chemoresistance in multiple ALL derived cell lines ${ }^{17}$. These studies justify the immediate need to start investigating anti-MM therapies in an hypoxic environment. Although data about chemoresistance induced by hypoxia in case of $\mathrm{MM}$ is non-existent, $\mathrm{TH}-302$, an hypoxia targeting pro-drug is currently in a phase $1 / 2$ clinical trial for $\mathrm{MM}$ at the Dana Farber Cancer Institute.

In Chapter 1, we addressed the importance of bone marrow hypoxia by hypothesizing that hypoxia is a major factor responsible for decreasing NK cell anti-MM responses. As the bone marrow is an anatomically challenging site to directly measure oxygen levels, we relied on literature to get a hint of what could be a representative oxygen concentration to perform anti-MM NK cell efficacy studies. In one of these studies, expression of HIF- $1 \alpha$ was observed in bone biopsies of MM patients ${ }^{18}$. As HIF-1 $\alpha$ is 
expressed when oxygen concentration is below $1.3 \%$, we performed NK cell functional assays over a range of oxygen concentrations between $0 \%$ and $1 \% \mathrm{O}_{2}$. Furthermore, in vivo, the hypoxic niches within the bone marrow are not uniform but rather heterogeneous with respect to their oxygen distribution ${ }^{19}$, and these hypoxic sites probably provide a residence to hematopoietic stem cells. We showed that the most important function of NK cells, that is the property to lyse target tumor cells, was almost completely lost when the cytotoxicity assay was performed under hypoxic conditions between $0 \%$ and $1 \% \mathrm{O}_{2}$. The phenomenon was stronger under the anoxic condition of $0 \% \mathrm{O}_{2}$, and there was hardly any NK cell induced $\mathrm{MM}$ cell killing. Our observation that the oxygen concentration at the time of kill was the most important factor determining NK cell lysis highlights the detrimental effect of hypoxia on NK cytotoxicity and also illustrates that a period of NK cell adaptation to hypoxia was not required for the NK cell restraining effects. Thus, the results would point towards a direction where $\mathrm{MM}$ cells can hide in hypoxic areas of the bone marrow thereby being protected from NK cells in general. Thus, to develop successful NK cell anti-MM therapy investigation of anti-MM responses under in vitro, hypoxic conditions is required.

Two previous studies, one with mouse and another with human MHC class I deficient leukemia cell lines had contrasting observations on the effect of hypoxia on NK cell killing. While one study showed $0 \% \mathrm{O}_{2}$ and not $1 \% \mathrm{O}_{2}$ reduced $\mathrm{NK}$ cell lysis against the mouse MHC negative tumor cell line YAC-120, the other second study demonstrated that $1 \% \mathrm{O}_{2}$ was enough to diminish NK cell cytotoxicity against $\mathrm{K} 56221$. Both cell lines are known to be easily killed by NK cells due to lack of MHC expression. In contrast, in Chapter 2, we used a series of MHC class I expressing MM cell lines, since the presence of MHC class I molecules on tumor cell is an important inhibitory factor for NK cells and an integral component regulating sensitivity of tumor cells for NK cells. Thus, with this study, we extend the initial observation of hypoxia induced NK cell impairment in the context of the missing self hypothesis, where the inhibitory signals provided by MHC class I are the key fine tuners of NK cell activity.

A limitation of our study was that we did not demonstrate the effect of hypoxia on NK cells with primary, patient derived MM cells. Primary patient derived MM cells die very rapidly in vitro, as they require a large number of additional growth factors, cytokines and stromal components of the bone marrow environment for them to survive in vitro after isolation from patients. Thus, due to lack of adequate culture systems available, we were unfortunately unable to test the effect of hypoxic NK cells on primary MM samples. Nevertheless, our observation that hypoxia alone has an inhibitory effect on NK cell mediated killing of MHC class I positive MM cells has recently also been observed for NK cells targeting the MHC class I positive breast cancer cell line MCF-7 by Baginska et al. In this study, it has been suggested that autophagy protects the breast cancer cell by degradation of granzyme B within the solid tumor cells ${ }^{22}$. 
Another effect of hypoxia that we investigated in Chapter 2 was its effect on NK cell activating and inhibitory receptors. Long-term chronic hypoxia could be detrimental for success for NK cell functionality in vivo if NK cells alter their expression of activating and inhibitory receptors. We observed that short-term exposure to hypoxia, 16 hours, already decreased the surface expression of two of the most important activating receptors, NKG2D and CD16. Moretta et al. recently observed a similar phenomenon for NKG2D and other activating receptors under long-term, 96 hours, hypoxic IL-2 NK cell cultures ${ }^{23}$. This preliminary observation could be of significant, clinical importance during future NK cell clinical trials. Further research is necessary to investigate methods to stabilize NK cell activating receptor expression under hypoxia. That way, NK cells injected in patients should retain their activating phenotype in vivo, and are not rendered nonfunctional by exposure to the long-term hypoxic microenvironment.

One approach to overcome this inhibition is to pre-activate the NK cells with IL-2. We found no significant difference between IL-2 activated NK cell killing either under hypoxia or normoxia. In addition in both conditions, the killing of MM cells by NK cells was dramatically improved upon IL-2 activation (Chapter 2). In this study we learned, that for effective NK cell therapy, a cytokine induced pre-activation of NK cells is required to boost their cytolytic potential to target hypoxic MM cells. Similarly, we would suggest if other MM supporting factors such when stromal cells also provide NK cell resistance, the approach of cytokine pre-activation needs investigation on each factor. A question still remaining is how long the IL-2 pre-activated NK cells are functional in vivo. IL-2 injections into patients during NK cell therapy is not recommended in the wake of studies showing IL-2 promotes undesirable regulatory $\mathrm{T}$ cell increase in vivo ${ }^{24}$. Thus to overcome this hurdle, it may require cell engineering approaches to genetically integrate the IL-2 gene in clinical grade NK cells.

\section{Selection of NK cells - Overcoming MM cell surface molecules mediated inhibition}

In our pursuit of developing NK cell therapy, the next step was to evaluate the resistance provided by MM cells though expression of cell surface HLA class I and HLAE. Carbone et al. have previously described heterogeneity in HLA-class I expression on the primary MM cell surface ${ }^{11}$. In Chapter 3, we also observed HLA-class I and HLA-E expression on $\mathrm{MM}$ cells obtained from patients. As these are molecules on the MM cell surface that are known to inhibit NK cell functionality, it is necessary to devise methods by which NK cells can overcome this inhibition during therapeutic application.

However, when we started this investigation, we encountered the paradox wherein MM cell lines strongly expressed HLA-class I, but HLA-E expression was lower than on primary MM cells. We hypothesized that this was due to bone marrow factors that are absent in in vitro culture systems. To test this, we injected the HLA-E ${ }^{\text {low }}$ U266 cell line 
intravenously in RAG $-2^{-1-} \mathrm{Yc}^{-/-}$mice. On isolation of cells from the bone marrow, HLA-E expression on U266 cells was upregulated in vivo, thus supporting our hypothesis and again demonstrating that in vitro models are limited to predict for in vivo responses.

Once we confirmed the presence of inhibitory cell surface molecules on MM cells in vivo, the next goal was determine their inhibitory potential using a NK-MM co-culture system, which allowed the simultaneous analysis of degranulation of different NK cell subsets. NK cells in peripheral blood circulation can express one or more of the KIRs with or without NKG2A, thus giving rise to at least sixteen NK cell subpopulations based on these markers. KIR but not NKG2A expressing NK cells can be broadly categorized into three NK cell subsets: i) NK cells which are completely matched for their KIR HLA class I interaction, ii) NK cells which are completely mismatched matched for their KIR HLA class I interaction, and iii) NK cells which have a coexpression of both matched and mismatched KIR's with respect to their HLA class I interaction. As expected, unactivated NK cells that were matched were unable to degranulate, while KIR - HLA class I mismatched NK cells could degranulate to a significantly higher extent. This phenomenon was even more pronounced for IL-2 activated NK cells. Presence of even one matched KIR on the NK cell surface diminished the degranulation potential of NK cells, suggesting that clinically NK cells that are completely mismatched for their KIR HLA class I interaction are the therapeutically most potent NK cell subsets. To increase the numbers of these KIR-ligand mismatched NK cells to be used in NK cell therapy, we would suggest to isolate and expand the KIR-ligand mismatched NK cell subset, prior to infusion. This procedure needs to be further investigated as it bears the risk of unwanted hyperactivity towards healthy cells or even NK derived neoplasia. An alternative approach would be to isolate NK cells from a "suitable" allogeneic donor, expand the number of total NK cells ex vivo, pre-activate them with IL-2 prior to infusion (as shown in Chapter 2), and then infuse higher numbers of total NK cells to patients, thereby achieving a higher number of KIR HLA class I mismatched NK cells that will have the proper potency to kill. When the total pool of NK cells is infused in patients, a large proportion of the NK cells would be KIR HLA class I ligand matched. This population of NK cells, although having a lower NK cell lytic potential (Chapter 3), is also an important population of NK cells as they may still provide help in elimination of tumor populations despite their lower degranulation capacity. Furthermore, it is not unrealistic to envision that high numbers of the KIR HLA class I ligand matched NK cells could sensitize the MM tumor cells for further lysis as a bystanding effect on KIR HLA class I mismatched NK cells.

In this study, we next investigated the inhibitory potential of MM cell surface HLA-E on NK killing activity (Chapter 3). Blocking HLA-E on MM cell lines enhanced degranulation of KIR-NKG2A+ NK cells, suggesting that even low levels of HLA-E on MM cell surface could be a potential barrier for NK cell cytotoxicity of NKG2A+ subsets. The experiment 
where high HLA-E expression was induced on MM cell lines by co-incubating, and thereby stabilizing, with HLA-E binding peptides, significantly dampened the response of KIR-NKG2A+ NK cells. These two observations suggest the NK cell impairing capacity of HLA-E during potential therapeutic intervention. One strategy in clinical practice would be to reduce HLA-E surface expression on MM cells. Since HLA-E expression is directly dependent of HLA class I expression, HLA-E downregulation can be achieved by conjugating NK cell therapy with immunomodulatory anti-MM chemotherapeutics, such as Bortezomib, which has been demonstrated to downregulate HLA class I expression on the MM cell surface ${ }^{25}$ and making the tumor cells more sensitive to NK cell lysis $^{26}$.

To summarize, in Chapter 3, we showed the inhibitory potential of HLA-class I and HLAE during their interaction with NK cells. It is thus necessary to overcome these inhibitory signals by careful selection of alloreactive NK cell subsets. Careful selection criteria would involve a "suitable" allogeneic NK cell donor, a donor with high number of KIR HLA class I mismatched NK cell in relation to a given patient. Subsequent to this, NK cells should be expanded in cytokine containing medium, and downstream elimination of NKG2A+ NK cells is desirable to achieve potentially maximum therapeutic benefit during therapy.

This selection process has, however, its limitations for MM patients with the genotype $\mathrm{C} 1+, \mathrm{C} 2+$ and $\mathrm{Bw} 4+$ as for these patients all the KIR receptors are always matched, and enhanced NK cell killing is never possible based on NK cell alloreactive subsets. For such patients to be still able to receive the proposed therapy, KIR HLA class I ligand matched NK cells are the only option. Furthermore in these patients, NK cell therapy could be combined with immunomodulatory drugs like lenalidomide, which has been shown to increase the surface expression of NKG2D ligands like MICA/B, ULBP1- $6^{27}$. Treatment with the HDAC inhibitor valproic acid has also been shown to enhance MICA/B expression in human osteosarcoma ${ }^{28}$. Another therapeutic strategy would be to treat such triple positive MM patients undergoing autologous NK cell therapy with anti-KIR antibodies $^{29}$. IPH2101 is a pan-specific anti-KIR antibody that recognizes KIR2DL1 and KIR2DL2/3. Romagne et al. demonstrated that this antibody improves the cytolytic potential of NK cells in HLA-C positive tumor cells ${ }^{30}$. A phase I clinical trial in MM with $\mathrm{IPH} 2101$ has demonstrated the safety of this antibody while ex vivo patient derived NK cells showed improved effector function ${ }^{31}$.

\section{In vivo activity of allogeneic NK cell therapy for eliminating MM}

Our group has previously shown in the 4T1 breast cancer model, that only a combination of chemo-/radiotherapy with allogeneic mice NK cells could efficiently cure these mice from breast cancer ${ }^{7}$. Thus, after establishing the anti-MM potential of allogeneic human NK cell in vitro (Chapter 3), our next aim was to investigate the anti- 
MM potential of allogeneic NK cells in vivo. This is an important bridging step towards clinical application of NK cell therapy for MM, because the bone marrow microenvironment with its complex structure of accessory cells, cytokines and growth factors can further provide resistance to NK cells in vivo. As the RAG2 ${ }^{-1-} \mathrm{yc}^{-1-}$ mice lack NK, B and T cells, it is possible to test cell therapy protocols with human MM cells and human NK cells in mice. We used this mouse model ${ }^{32}$ to study the tumor growth kinetics of the GFP+ Luciferase+ U266 human MM cell line after our therapeutic interventions. Four therapeutic protocols were investigated with this system in a sequential order, the effect of: i) unactivated allogeneic NK cells alone, ii) IL-2 activated allogeneic NK cell therapy, iii) chemo-/radiotherapy without NK cell infusion, and iv) IL2 activated allogeneic NK cell therapy in combination with chemo-/radiotherapy. As the U266 cell line is HLA C1+, alloreactivity in all experiments was achieved by selecting donors whose NK cells were KIR2DL1 and/or KIR3DL1 positive. The percentage of NK cells that were NKG2A negative and completely mismatched for KIR HLA class I interaction in vivo ranged from $4-17 \%$ in different donors.

In the first experimental protocol tested, different numbers of non-activated allogeneic NK cells were injected. Only in the group receiving the highest number of NK cells ( 3 injections with $8 \times 10^{6} \mathrm{NK}$ cells), one of four mice showed a reduced tumor burden upon treatment with human NK cells. This demonstrated the proof of concept that NK cells can have anti-MM activity in the model. We therefore used this dosage of NK cells in all subsequent NK cell in vivo experiments. Combining the knowledge gained from our initial studies in Chapter 2 and 3, we knew that IL-2 activated alloreactive NK cells can be potent effector cells in targeting hypoxic MM cells. Thus, in the next in vivo study, we investigated the anti-MM response of IL-2 activated allogeneic NK cells. Although there was no complete cure against MM using IL-2 activated NK cells but a growth delay was induced, we could demonstrate the presence of injected human NK cells circulating in blood, upto 3 weeks post injection, and at week 7 (the human endpoint for this experiment) in the bone marrow. This is a significant finding showing the effective migration and homing of IL-2 NK cells in the RAG2 ${ }^{-/-} \mathrm{yc}^{-{ }^{-}}$mice $\mathrm{MM}$ model.

The combination of chemo-/radiotherapy alone did not significantly improved progression free survival in the RAG2 ${ }^{-1-} \mathrm{yc}^{-/-} \mathrm{MM}$ model. However, bioluminescence imaging revealed significant curative effect progression free survival of IL-2 activated NK cell therapy together with intraperitoneal doses of IL-15, over and above chemo/radiotherapy.

In summary, we showed a clear biological effect of donor NK cells in combination with chemo-/radiotherapy in the RAG2 ${ }^{-/} \mathrm{yc}^{-/-} \mathrm{MM}$ mouse model. This will pave the way for further experiments to optimize the therapy to reach an effective cure rate. Another important observation of our study is the absence of GVHD in mice receiving allogeneic 
NK cells. This provides future opportunities to further boost NK cell activity in vivo, without the side effects of GHVD.

\section{Immunomodulatory boosting of NK cell MM activity by combination with new generation chemotherapeutics or monoclonal antibodies}

After having addressed the inhibitory factors at the MM cellular and microenvironmental level, and having observed an improvement in survival of MM bearing mice, the results were suggestive for possible NK cell therapy for MM patients. However, in vivo we observed that NK cells reduced the MM burden, but, after a long time period, the tumor progressed (Chapter 4). Thus, in Chapter 5, we aimed to identify strategies that could further enhance NK cell anti-MM also in an hypoxic environment. To this end, we tested in our hypoxic in vitro culture system several drugs (lenalidomide $^{27,33,34}$, bortezomib ${ }^{25,26}$, BIIB-021 and 7-AAG ${ }^{35}$ and a monoclonal antibody (CD229) that have been described to boost NK cell activity under normoxic culture conditions. In line with literature ${ }^{36}$, the combination of NK cells with anti-CD229, an antibody towards a surface antigen expressed on MM cells, indeed sensitized MM to NK cell killing under normoxia. Unfortunately, this antibody failed to improve NK mediated MM killing under hypoxia in different effector to target ratios (Chapter 5). We next tested another widely investigated immunomodulatory chemotherapeutic, lenalidomide ${ }^{37,38}$ to sensitize $\mathrm{MM}$ cells ${ }^{27}$, but this treatment also failed to improve NK killing in an hypoxic environment. Also MM pretreatment with bortezomib, BIIB-021 and 17-AAG did not make the target cells more susceptible for killiing by NK cells. These observations suggest that hypoxia can provide a sanctuary for therapy resistant cells. Thus, other immunomodulatory therapeutics for MM should be tested in the hypoxic environment to identify agents and treatment strategies that are effective in an hypoxic environment. Alternatively, these immunomodulatory drugs could be used in combination with hypoxia inhibiting drugs, such as $\mathrm{TH}-302^{39}$. TH-302 has been shown to selectively kill MM cells in an hypoxic environment. Optimal combination of $\mathrm{TH}-302$ and a second wave of NK cell therapy during treatment may further improve progression free survival, and allow the successful development of NK cell therapy for MM, but remains to be investigated.

All together, we have indicated in the work described in this thesis that there might be a future for donor NK cell therapy in MM patients, either to prolong the life of these patients or to potentially cure them. Since NK cell therapy is a completely different approach than chemotherapy, where there is ample evidence that cancer cells residing in the hypoxic areas of tumors are therefore less responsive to these drugs, most likely combinations of treatment procedures (NK cell therapy and drugs that are active in hypoxic areas) will have the best clinical outcome since we showed here that IL-2 activated NK cells surely can kill under hypoxic conditions. 


\section{Future prospects}

The work in this thesis has shown a potential treatment regimen of NK cell therapy in MM in combination with chemo-/radiotherapy. It also showed that in murine models no complete cure was achieved yet, so work on these levels has to be continued. Moreover, synergistic or additive effector mechanisms have to be established.

There are currently several drugs under investigation that may have a beneficial effect to kill MM cells either by targeting the tumor cells directly (interference in the cellular pathway of the MM cells) or inhibiting the stromal cells and/or cytokines relevant for tumor growth ${ }^{40,41}$. As mentioned already earlier, a combination treatment that is very interesting to test is a combination of donor NK cells and monoclonal antibodies. Not only anti-CD229 is an option, but as in Non-Hodgkin Lymphoma (NHL), another hematological malignancy, an antibody directed against CD20 on the tumor cells has long been demonstrated to be effective in patients. It improves survival and cure rate in some types of $\mathrm{NHL}^{42}$. It is expected that in the near future, antibodies directed to $\mathrm{MM}$ will also be registered for clinical use. These antibodies will attract more complement, neutrophils or NK cells to the cancerous cells - the latter all binding to the antibody -, which then contribute to the killing of the tumor. Since in this thesis we clearly demonstrated that allogeneic NK cells, partly by the missing self concept, have an antiMM effect, we are very interested in investigating such antibodies in combination with donor NK cells. Unfortunately, thus far we have not been able to bring this to the test due to lack of availability of the antibodies.

Several antibodies are under development that may boost NK cell effector functions by interfering with suppressive factors on NK cells, the so-called immune checkpoint blockade. Benson et al. showed that NK cells from MM patients express PD-1 whereas normal NK cells do not ${ }^{43}$. It is known that the interaction of PD-1 with PD-L1 decreases NK cell cytotoxicity. CT-011 is a novel anti-PD-1 antibody that enhances human NK cell function against autologous primary $M M$ cells by interfering with the PD-1/PD-L1 interaction $^{33}$. Another example is to block inhibitory proteins on NK cells, like NKG2A (IPH2201, Innate Pharma) or HLA class I (IPH2010, Innate Pharma-BMS). By using this antibody, it is being tested whether NK cells become more functional against tumor cells that are not killed due to high expression levels of HLA-E. Therefore blocking the HLA-E - NKG2A receptor interaction should lead to increased NK cell activity.

Several disadvantages exist to our approach of developing donor cell therapy for MM. First of all, by making use of donor cells - that will not be accepted by the patient's immune system - a conditioning regimen is needed that allows donor NK cells to be not rejected and migrate in the patient. However, since we already demonstrated that especially the combination of chemo-/radiotherapy - that will allow for immunosuppression and therefore immunological acceptance of the donor cells - we 
expect this to be a possible advantage for the patient as well. These regimens may have a certain risk because of toxicity, however, probably have a high acceptance rate since the treatment will be used in patients that will not have a long survival without other treatment options.

A research question that remains to be investigated is what dose of NK cells is safe for patients, without causing severe side effects. In a recent study, Choi et al. performed a dose escalation study of donor derived NK cells after HLA haploidentical hematopoietic cell transplantation (HCT) and found that a dose of $2 \times 10^{8}$ cells $/ \mathrm{kg}$ of body weight is well tolerated when given after 2-3 weeks after $\mathrm{HCT}^{44}$. Thus, protocols to upscale the generation of high NK cell numbers for clinical application is another parameter that we are currently investigating in our lab.

Developing cellular therapy protocols will require a high technical demand to culture these cells. Because of international regulations, these cells have to be cultured under Clinical Manufacturing Procedures (GMP), and can only be performed in certain laboratory facilities. To culture high enough numbers of cells under these GMP guidelines will make the procedures time consuming and expensive. Preliminary results in our laboratory - together with Zellwerk, a company that provides a special bioreactor to culture large numbers of NK cells - have indicated the possibility to culture accurate numbers of cells obtained from volunteers. The price of the product is calculated to be at least $€ 25.000$. Whether this is cost effective will be finally determined by the clinical effect of these cells and might be less than other procedures like donor stem cell transplantation ( $€$ 125.000) or certain drugs (above $€ 100.000$ per year) that are presently used. It surely is in the well-accepted range of costs that can be made for a gained quality of life year.

Presently in my position as post doc at the Dana Farber Cancer Institute, I am investigating the role of genetic changes in tumor suppressor genes $(p 53)^{45}$ and oncogenes (NRAS) (KRAS), which could potentially provide additional routes of NK cell evasion by $\mathrm{MM}$ cells. A recent study shows that $\mathrm{MM}$ cell populations from patients can by themselves harbor heterogeneous subclonal populations of these genetic alterations ${ }^{46}$. Thus, further research is required to identify the potency of NK cells in eliminating different clonal populations of mutated tumor suppressor or oncogenes. A better understanding of genetic alterations in $\mathrm{MM}$ cells, which can confer resistance to NK cells, will enable development of more personalized immunomodulatory NK cell therapy for MM patients harboring an extensively mutated genome. In addition, my current lab has demonstrated that the stromal compartment of MM confers resistance to NK cells ${ }^{47}$ and cytotoxic T lymphocytes (CTL's $)^{48}$. We now aim to identify novel immunomodulatory drugs that target both the stromal compartment and hypoxia, and also boost immune effector cell functions. 


\section{REFERENCES}

1. Lonial S, Boise LH (2013) The future of drug development and therapy in myeloma. Semin Oncol 40: 652-658.

2. Palumbo A, Attal M, Roussel M (2011) Shifts in the therapeutic paradigm for patients newly diagnosed with multiple myeloma: maintenance therapy and overall survival. Clin Cancer Res 17: 1253-1263.

3. Lu S, Wang J (2013) The resistance mechanisms of proteasome inhibitor bortezomib. Biomark Res 1: 13.

4. Ruggeri L, Capanni M, Urbani E, Perruccio K, Shlomchik WD, et al. (2002) Effectiveness of donor natural killer cell alloreactivity in mismatched hematopoietic transplants. Science 295: 2097-2100.

5. Kroger N, Shaw B, lacobelli S, Zabelina T, Peggs K, et al. (2005) Comparison between antithymocyte globulin and alemtuzumab and the possible impact of KIR-ligand mismatch after dose-reduced conditioning and unrelated stem cell transplantation in patients with multiple myeloma. $\mathrm{Br} \mathrm{J}$ Haematol 129: 631-643.

6. Vanclee A, van Gelder M, Schouten HC, Bos GM (2006) Graft-versus-tumor effects on murine mammary carcinoma in a model of nonmyeloablative haploidentical stem cell transplantation. Bone Marrow Transplant 37: 1043-1049.

7. Frings PW, Van Elssen CH, Wieten L, Matos C, Hupperets PS, et al. (2011) Elimination of the chemotherapy resistant subpopulation of $4 \mathrm{~T} 1$ mouse breast cancer by haploidentical NK cells cures the vast majority of mice. Breast Cancer Res Treat 130: 773-781.

8. Lokhorst HM, Schattenberg A, Cornelissen JJ, van Oers MH, Fibbe W, et al. (2000) Donor lymphocyte infusions for relapsed multiple myeloma after allogeneic stem-cell transplantation: predictive factors for response and long-term outcome. J Clin Oncol 18: 3031-3037.

9. Lokhorst HM, Wu K, Verdonck LF, Laterveer LL, van de Donk NW, et al. (2004) The occurrence of graftversus-host disease is the major predictive factor for response to donor lymphocyte infusions in multiple myeloma. Blood 103: 4362-4364.

10. Vasievich EA, Huang L (2011) The suppressive tumor microenvironment: a challenge in cancer immunotherapy. Mol Pharm 8: 635-641.

11. Carbone E, Neri P, Mesuraca M, Fulciniti MT, Otsuki T, et al. (2005) HLA class I, NKG2D, and natural cytotoxicity receptors regulate multiple myeloma cell recognition by natural killer cells. Blood $105:$ 251-258.

12. Fauriat C, Mallet F, Olive D, Costello RT (2006) Impaired activating receptor expression pattern in natural killer cells from patients with multiple myeloma. Leukemia 20: 732-733.

13. El-Sherbiny YM, Meade JL, Holmes TD, McGonagle D, Mackie SL, et al. (2007) The requirement for DNAM-1, NKG2D, and NKp46 in the natural killer cell-mediated killing of myeloma cells. Cancer Res 67: 8444-8449.

14. Frassanito MA, Silvestris F, Cafforio P, Silvestris N, Dammacco F (1997) IgG M-components in active myeloma patients induce a down-regulation of natural killer cell activity. Int J Clin Lab Res 27: 48-54.

15. Selvendiran K, Bratasz A, Kuppusamy ML, Tazi MF, Rivera BK, et al. (2009) Hypoxia induces chemoresistance in ovarian cancer cells by activation of signal transducer and activator of transcription 3. Int J Cancer 125: 2198-2204.

16. Lara PC, Lloret M, Clavo B, Apolinario RM, Henriquez-Hernandez LA, et al. (2009) Severe hypoxia induces chemo-resistance in clinical cervical tumors through MVP over-expression. Radiat Oncol 4: 29.

17. Benito J, Shi Y, Szymanska B, Carol H, Boehm I, et al. (2011) Pronounced hypoxia in models of murine and human leukemia: high efficacy of hypoxia-activated prodrug PR-104. PLoS One 6: e23108.

18. Colla S, Storti P, Donofrio G, Todoerti K, Bolzoni M, et al. (2010) Low bone marrow oxygen tension and hypoxia-inducible factor-1alpha overexpression characterize patients with multiple myeloma: role on the transcriptional and proangiogenic profiles of CD138(+) cells. Leukemia 24: 1967-1970.

19. Parmar K, Mauch P, Vergilio JA, Sackstein R, Down JD (2007) Distribution of hematopoietic stem cells in the bone marrow according to regional hypoxia. Proc Natl Acad Sci U S A 104: 5431-5436.

20. Loeffler DA, Juneau PL, Heppner GH (1991) Natural killer-cell activity under conditions reflective of tumor micro-environment. Int J Cancer 48: 895-899. 
21. Fink T, Ebbesen P, Koppelhus U, Zachar V (2003) Natural killer cell-mediated basal and interferonenhanced cytotoxicity against liver cancer cells is significantly impaired under in vivo oxygen conditions. Scand J Immunol 58: 607-612.

22. Baginska J, Viry E, Berchem G, Poli A, Noman MZ, et al. (2013) Granzyme B degradation by autophagy decreases tumor cell susceptibility to natural killer-mediated lysis under hypoxia. Proc Natl Acad Sci U S A 110: 17450-17455.

23. Balsamo M, Manzini C, Pietra G, Raggi F, Blengio F, et al. (2013) Hypoxia downregulates the expression of activating receptors involved in NK-cell-mediated target cell killing without affecting ADCC. Eur J Immunol 43: 2756-2764.

24. Ahmadzadeh M, Rosenberg SA (2006) IL-2 administration increases CD4+ CD25(hi) Foxp3+ regulatory T cells in cancer patients. Blood 107: 2409-2414.

25. Shi J, Tricot GJ, Garg TK, Malaviarachchi PA, Szmania SM, et al. (2008) Bortezomib down-regulates the cell-surface expression of HLA class I and enhances natural killer cell-mediated lysis of myeloma. Blood 111: 1309-1317.

26. Hallett WH, Ames E, Motarjemi M, Barao I, Shanker A, et al. (2008) Sensitization of tumor cells to NK cell-mediated killing by proteasome inhibition. J Immunol 180: 163-170.

27. Benson DM, Jr., Bakan CE, Zhang S, Collins SM, Liang J, et al. (2011) IPH2101, a novel anti-inhibitory KIR antibody, and lenalidomide combine to enhance the natural killer cell versus multiple myeloma effect. Blood 118: 6387-6391.

28. Yamanegi K, Yamane J, Kobayashi K, Kato-Kogoe N, Ohyama H, et al. (2010) Sodium valproate, a histone deacetylase inhibitor, augments the expression of cell-surface NKG2D ligands, MICA/B, without increasing their soluble forms to enhance susceptibility of human osteosarcoma cells to NK cellmediated cytotoxicity. Oncol Rep 24: 1621-1627.

29. Alici E (2010) IPH-2101, a fully human anti-NK-cell inhibitory receptor mAb for the potential treatment of hematological cancers. Curr Opin Mol Ther 12: 724-733.

30. Romagne F, Andre P, Spee P, Zahn S, Anfossi N, et al. (2009) Preclinical characterization of 1-7F9, a novel human anti-KIR receptor therapeutic antibody that augments natural killer-mediated killing of tumor cells. Blood 114: 2667-2677.

31. Benson DM, Jr., Hofmeister CC, Padmanabhan S, Suvannasankha A, Jagannath S, et al. (2012) A phase 1 trial of the anti-KIR antibody IPH2101 in patients with relapsed/refractory multiple myeloma. Blood 120: 4324-4333.

32. Rozemuller H, van der Spek E, Bogers-Boer LH, Zwart MC, Verweij V, et al. (2008) A bioluminescence imaging based in vivo model for preclinical testing of novel cellular immunotherapy strategies to improve the graft-versus-myeloma effect. Haematologica 93: 1049-1057.

33. Galustian C, Labarthe MC, Bartlett JB, Dalgleish AG (2004) Thalidomide-derived immunomodulatory drugs as therapeutic agents. Expert Opin Biol Ther 4: 1963-1970.

34. Guglielmelli T, Petrucci MT, Saglio G, Palumbo A (2011) Thalidomide after lenalidomide: a possible treatment regimen in relapse refractory multiple myeloma patients. Br J Haematol 152: 108-110.

35. Fionda C, Soriani A, Malgarini G, lannitto ML, Santoni A, et al. (2009) Heat shock protein-90 inhibitors increase $\mathrm{MHC}$ class I-related chain A and B ligand expression on multiple myeloma cells and their ability to trigger NK cell degranulation. J Immunol 183: 4385-4394.

36. Atanackovic D, Panse J, Hildebrandt Y, Jadczak A, Kobold S, et al. (2011) Surface molecule CD229 as a novel target for the diagnosis and treatment of multiple myeloma. Haematologica 96: 1512-1520.

37. Hsu AK, Quach H, Tai T, Prince HM, Harrison SJ, et al. (2011) The immunostimulatory effect of lenalidomide on NK-cell function is profoundly inhibited by concurrent dexamethasone therapy. Blood 117: 1605-1613.

38. Bartlett JB, Michael A, Clarke IA, Dredge K, Nicholson S, et al. (2004) Phase I study to determine the safety, tolerability and immunostimulatory activity of thalidomide analogue CC-5013 in patients with metastatic malignant melanoma and other advanced cancers. Br J Cancer 90: 955-961.

39. Hu J, Handisides DR, Van Valckenborgh E, De Raeve H, Menu E, et al. (2010) Targeting the multiple myeloma hypoxic niche with TH-302, a hypoxia-activated prodrug. Blood 116: 1524-1527.

40. Raje N, Hideshima T, Mukherjee S, Raab M, Vallet S, et al. (2009) Preclinical activity of P276-00, a novel small-molecule cyclin-dependent kinase inhibitor in the therapy of multiple myeloma. Leukemia 23 : 961-970. 
41. Podar K, Chauhan D, Anderson KC (2009) Bone marrow microenvironment and the identification of new targets for myeloma therapy. Leukemia 23: 10-24.

42. Rehwald U, Schulz H, Reiser M, Sieber M, Staak JO, et al. (2003) Treatment of relapsed CD20+ Hodgkin lymphoma with the monoclonal antibody rituximab is effective and well tolerated: results of a phase 2 trial of the German Hodgkin Lymphoma Study Group. Blood 101: 420-424.

43. Benson DM, Jr., Bakan CE, Mishra A, Hofmeister CC, Efebera Y, et al. (2010) The PD-1/PD-L1 axis modulates the natural killer cell versus multiple myeloma effect: a therapeutic target for CT-011, a novel monoclonal anti-PD-1 antibody. Blood 116: 2286-2294.

44. Choi I, Yoon SR, Park SY, Kim H, Jung SJ, et al. (2014) Donor-Derived Natural Killer Cells Infused after HLA-Haploidentical Hematopoietic Cell Transplantation: A Dose-Escalation Study. Biol Blood Marrow Transplant.

45. Neri A, Baldini L, Trecca D, Cro L, Polli E, et al. (1993) p53 gene mutations in multiple myeloma are associated with advanced forms of malignancy. Blood 81: 128-135.

46. Lohr JG, Stojanov P, Carter SL, Cruz-Gordillo P, Lawrence MS, et al. (2014) Widespread genetic heterogeneity in multiple myeloma: implications for targeted therapy. Cancer Cell 25: 91-101.

47. McMillin DW, Delmore J, Negri JM, Vanneman M, Koyama S, et al. (2012) Compartment-Specific Bioluminescence Imaging platform for the high-throughput evaluation of antitumor immune function. Blood 119: e131-138.

48. de Haart SJ, van de Donk NW, Minnema MC, Huang JH, Aarts-Riemens T, et al. (2013) Accessory cells of the microenvironment protect multiple myeloma from T-cell cytotoxicity through cell adhesionmediated immune resistance. Clin Cancer Res 19: 5591-5601. 
Summary 


\section{Summary}

Multiple Myeloma accounts for about $13 \%$ of the hematological cancers and is responsible for $0.9-1.2 \%$ of deaths from cancer. Most people are above the age of 65 when the diagnosis is made. After patients are diagnosed with Multiple Myeloma, they are treated with a combination of frontline chemotherapy, which includes a variety of drugs. Thus far, there is no option to cure Multiple Myeloma. Therefore, the effort is directed to improve overall survival and maintaining quality of life. However, in the last decade, several drugs, like bortezomib, thalidomide and lenalidomide, have become available that due to a better anti-myeloma effect improve survival of the patients.

The precise combination of drugs to be used is depending on age and comorbidity. Eligible patients with a healthy immune status are treated with a regimen that aims for reduction in myeloma burden and after this induction treatment high dose chemotherapy will follow. An important step prior to this chemotherapeutic procedure is to isolate the patients hematopoietic stem cells (HSCs) and freeze them, since the intensive chemotherapy treatment will not only destroy tumor cells, but also healthy bone marrow stem cells that are needed to produce all blood cells. Therefore, the high dose chemotherapy will be followed by a return of the frozen HSCs, a procedure called autologous stem cell transplantation (auto-SCT). Several clinical trials have indicated that this procedure improves overall survival. Despite this intensive treatment schedule, patients will not be cured, though some patients show a long period (years) of progression free survival with good quality of life.

Allogeneic SCT (allo-SCT) is an alternative to auto-SCT. By making use of donor stem cells, one can transplant a donor immune system to the recipient patient. This procedure, that was originally developed to compensate for radiation damage by which peoples' bone marrow stem cells were destroyed, has the potential to cure malignant diseases by the so called graft (=donor) versus disease response of the immune system. However, this procedure can also induce an unwanted graft versus host effect (GVH) with detrimental effects on healthy organs, mostly skin, gut, liver and lungs. This GVH effect is due to the fact that donors that are selected will still be mismatched for (minor) histocompatibility antigens that evoke the donor immune system to respond.

Since the limitations of the clinical outcome of allo-SCT and the scarcity of accurately matched donors to perform an allo-SCT as described above on time, investigators have looked for alternative donors. The use of haploidentical donors, sharing one of the two haplotypes with the patient can considerably overcome this problem of donor availability. Every patient will usually have a donor since parents, siblings, children and some nieces/nephews will share one haplotype (a set of chromosomes with the relevant transplantation antigens, coming from the father or mother) with the patient. 
Ruggeri et al. also showed in a landmark paper the phenomenon of enhanced graft vs acute myeloid leukemia (AML) response during haploidentical stem cell transplantation. Surprisingly, the clinical anti-tumor effect was probably due to graft versus tumor effect of the NK cells and not due to T cells. Our group has shown that haploidentical stem cell transplantation together with nonmyeloablative conditioning regimen can cure breast cancer in a 4T1 breast cancer mice model. Subsequent experiments by our group identified donor NK cells as crucial for the graft versus tumor effect during haploidentical transplantation in this model. NK cell depletion of the haploidentical graft fully abrogated the anti-tumor effect and only NK cells in the absence of donor stem cells demonstrated similar results, confirming the data of Ruggeri et al. that NK cells from haploidentical donors might have the potency for strong anti-tumor effects, not only in hematological tumors, but also in solid tumors.

NK cells are large granular lymphocytes, which can selectively kill virally infected cells and tumor cells without affecting healthy normal cells. Certain NK cell subsets can mount direct cytotoxic action against virally infected or tumor cells. In Chapter 2, we show the need to perform in vitro investigations to study interactions between NK cells and hematological tumor cells under hypoxic conditions. Furthermore, our study provides proof of concept that activating cytokines can overcome the adverse effects of hypoxia in vitro and shows that hypoxia is a factor to take into account when designing allogeneic NK cell based immunotherapy for MM. Experimental set ups comparable to ours will be helpful to determine the potential of novel and existing cytokines or immunomodulatory agents, to boost NK cell responses in a way that NK cells can also exert their effector function in the presence of tumor environmental factors like hypoxia. For future clinical perspectives it might be considered to combine NK cell therapy with hypoxia-targeting and pre-activation of NK cells to eliminate tumor cells in a hypoxic environment.

Immunotherapy with allogeneic NK cells offers therapeutic perspectives for Multiple Myeloma patients. In Chapter 3, we aimed to refine NK cell therapy by evaluation of the relevance of HLA-class I and HLA-E for NK-anti-myeloma reactivity. Our results show that KIR-HLA-class I and NKG2A-HLA-E interactions are highly relevant for NK cell reactivity against myeloma. For accurate prediction of in vitro data to patient's reality, two relevant in vivo realities have to be taken into account: 1 ) in the bone marrow myeloma cells reside under hypoxic conditions and 2) in vivo, myeloma cells express both HLA-class I and HLA-E. Infusion of a high number of cytokine activated alloreactive NKG2A negative, KIR-ligand mismatched NK cells may help to improve the efficacy of alloreactive NK cell therapy. Our study demonstrates that NKG2A-negative, KIR-ligand mismatched NK cells are the most potent subset for clinical application. We envision that infusion of high numbers of this subclass will enhance clinical efficacy. 
In Chapter 4, we explored the activity of alloreactive Natural Killer (NK) cells after chemo-radiotherapy conditioning as a novel immunotherapy treatment option for Multiple Myeloma (MM). Using the RAG2 ${ }^{-1-} \mathrm{yc}^{-/-}$immunodeficient disseminated MM mouse model, we studied NK cell mediated Graft versus MM (GvM). Our study illustrates the therapeutic potential and safety of NK cell therapy in MM and is an important step towards cell-based immunotherapy for $\mathrm{MM}$ patients without the induction of GVHD. It also establishes the RAG2 ${ }^{-/} \mathrm{Yc}^{-/-} \mathrm{MM}$ model as a clinically relevant platform to further develop NK cell based immunotherapy. We showed the biological anti-MM potential of alloreactive NK cells against disseminated MM. In addition, we demonstrate that IL-2 activated NK cells can home to the bone marrow of MM bearing mice and that anti-MM responses occurred in a dose dependent manner. The latter suggests that infusion of a higher number of alloreactive NK cells can help to improve clinical efficacy and that patients should, preferably, be treated in remission or at least with low residual disease. Our data also demonstrate that combination therapy of alloreactive NK cells and chemo/radiotherapy could help to improve the anti-MM effect.

Success of new generation anti-MM drugs over the past decade has raised hopes for treating Myeloma. Discovery of new generation chemotherapeutics and monoclonal antibodies (MoAb's) for MM have significantly improved overall survival in the last decade. These novel drugs have demonstrated to display immunomodulatory (IMD) effects on NK cells in vitro or trigger antibody dependent cellular cytotoxicity (ADCC). We therefore hypothesized in Chapter 5 that combining these drugs with NK cell therapy may provide a successful approach for the treatment of MM. We observed that MM cells treated with an array of different IMD anti-MM drugs or MAb's, and cultured under hypoxia ( $1 \%$ or $0.2 \% \mathrm{O}_{2}$ ) were not enhancing NK cell cytotoxicity as compared to untreated cells. This study demonstrates the necessity of in vitro anti-myeloma therapeutic research at hypoxic oxygen levels representative of bone marrow environment to be able to draw the right conclusions about new possibilities.

All together, we have demonstrated in the work described in this thesis that there might be a future for donor NK cell therapy in MM patients to potentially cure them. Since NK cell therapy is a completely different approach than chemotherapy, where there is ample evidence that cancer cells residing in the hypoxic areas of tumors are therefore less responsive to these drugs, most likely combinations of treatment procedures (NK cell therapy and drugs that are active in hypoxic areas) will have the best clinical outcome since we showed here that IL-2 activated NK cells surely can kill under hypoxic conditions. 
Acknowledgement 


\section{Acknowledgement}

First of all, I am thankful to my supervisor, Prof. dr. Gerard Bos whose encouragement and support enabled me to develop an understanding of Multiple Myeloma. His clear vision about NK cell therapy for Myeloma was a key to the successful and timely completion of different projects. The biggest thing, which I have learned from him as a leader is how to be open as a listener to the team, and yet being able to remind and refocus the team towards the goal. My co-supervisors Dr. Wilfred Germeraad and Dr. Lotte Wieten have been instrumental in shaping my research skills in the past 4 years. They together taught me different aspects of immunology and flowcytometry. Also the social support from them during my stay in the Netherlands has been very warm. With both Wilfred and Lotte, the list of memories is too long, and it's beautiful. Right from the great car drives on the highways, cricket, NVVI's, my first day in the Netherlands, September 13, 2009, when Lotte drove me all the way from Utrecht to Maastricht.

I would also like to thank the members of the Internal Medicine department at MUMC+, Prof. dr. Harry Schouten and Dr. Michel van Gelder for their scientific input during the 4 years of PhD studies. Michel had the great skill/art to lighten up the start of every Monday morning meeting, which made the meeting productive and fun. Harry, your relaxed smile, is extremely relaxing, and always motivated me to stay relaxed.

I would to take this opportunity to thank the School of Oncology and Developmental Biology (GROW) Maastricht, for providing me with the opportunity to conduct my PhD research in the field of Tumor Immunology and Immunotherapy. The school has been instrumental in providing finances and timely guidance in different aspects of research.

The members of the thesis committee, Prof. dr. P. Lambin, Prof. dr. E.A.L. Biessen, Prof. dr. P.J. Peters, Prof. dr. K. Satyamoorthy and Prof. dr. S. Zweegman, thank you very much for reading and judging this thesis and allowing me to defend it.

I would like to thank all the donors who have volunteered to donate blood for my research multiple times over the past several years. This research would not have been possible without their support and enthusiasm.

I am highly grateful to all my external collaborators: Dr. Anton Martens, Dr. Richard Groen, and Dr.Willy Noort in Utrecht for helping me with the mice studies. During my month long stay in September 2013 in Utrecht, I came to know all of you more closely, and it was a pleasure to work with all three of you. Dr. Kasper Rouschop at Maastro lab for his advice on hypoxia related aspects of cancer, and helping me with the hypoxic 
chambers. He has always been very enthusiastic about my research work, which prompted a great deal of confidence in me time and again.

The lab environment was very friendly, and I made great friends: Mirelle, Tammy, Ans, Eveline, Thomas, Mateusz, Birgit, Melanie, Janine and Ariane. Birgit taught me some Dutch and French, which I will never forget: "Mag ik graag een buffy voor dinsdag". Melanie was a great person with a jolly nature. Mirelle and Tammy provided all the calories in the form of cakes and other baked products. Mirelle has been a great listener during the last two years; I wish you all the success you can get in your life. Project students, Mark, Lieneke and Florance were also very passionate about their research, which really made me enthusiastic as a researcher and teacher. Thank you for everything.

The team at Transplantation Immunology of the MUMC+, Prof Dr. Marcel Tilanus, Dr. Joris Vanderlocht, Dr. Nina Lauterbach, and Dr. Christien Voorter, has been a great support over the years. The time spend with them, professionally and socially during carnivals will always be a great memory. Thank you Joris for all the nice moments and memories we have made at your place with your family. Your presentation and communication skills at work have always impressed me a lot. It was great knowing you and hopeful to stay connected with you. Thank you Nina for all the carnival dresses, especially the red Indian one. I owe a big thanks to every member of the Transplantation Immunology group, who helped me in the process of cell collection over the past 4 years. This thesis would not have been possible at all without you all. I would especially like to express my heart-felt thanks to Annette for all her help.

Shilpi during my 4 years staying in the Netherlands, I became great friends with Mitrajit, Oindrilla, Subrata, Ajay, Sameera, Ramesh, and Kranthi. I always felt the warmth of their friendship. My stay in Aachen with Mitrajit and company was the best times in life while I was in Europe. Mitrajit, I think we have battled more than being at peace, which is the way I want to keep it in the future. The badminton shots that I played like Rafel Nadal every Friday, you did not really appreciate!! But watching the India v Australia World Cup final in 2011, April 2, in Aachen with a bunch of $>15$ great friends... the eruption of joy when we won... is my most memorable time in life. Oindrilla, you are a great listener. Oindrilla, you are a great listener. Oindrilla, you are a great listener!! Subrata, your dim-shedoo bhat with posto, was the reason why I frequently made a trip to Aachen.

I would like to thank my previous teachers who have been my role models over the years. My high school biology teacher, Subrata Roy, pioneered my interest in Biological Science. During my stay in Manipal, Dr. Ullas Kamath and Dr. Prakash PY were exceptional at their ability to infuse interest in the field of biochemistry and 
microbiology, respectively. Dr. Rajashekhar Moka and Dr. Muthusamy were a key mentor and have always encouraged me to concentrate on my studies and research. Dr. K. Satyamoorthy always made us believe in ourselves and made us confident scientists. It was his confidence in me that gave me the opportunity to pursue a $\mathrm{PhD}$ at Maastricht University. A special thanks to Dr. Constantine Mitsiades at Dana-Farber for the support that enabled me to complete my thesis writing during my first year at DFCl and also mentoring me in different aspects of drug resistance in Myeloma.

Finally, I would like to thank my parents for their support in life, and for being there in the tough times. It all started when I used to visit my dad's chemistry lab at the age of 5. Again, I think I have had more number of "fight days" than "peace days" with them, which to me was always indicative of the greatness of things are great. I really can't describe in words what they mean to me. All of my grand-parents, who are no more, but I know you are watching me, and I dedicate this work to all of you. Lotte and Wilfred have been by my side at times when things were turbulent. Again I don't have words to explain my thanks to them. These 4 years have been a great experience, I had a great share of joy, Eureka moments, and moments of sadness, moments of fighting, moments of laughter, and moments of "wine-cheese-bitterballen" and would like to thank everyone who has been a part of my journey. 
Curriculum Vitae 


\section{Curriculum vitae}

Subhashis Sarkar was born on May 1, 1986 at Kolkata, India. He did his Bachelor's and Master's study at Manipal Life Sciences Centre, Manipal. During this period he gained research experience on monoclonal antibodies used in cancer therapy, while doing his master's thesis project at Biocon Biopharmaceuticals. In May 2009 he was awarded the "Best Outgoing Student 2009- Manipal Life Sciences Centre" Subsequently he joined Maastricht University, Netherlands, in 2009 for pursuing his doctoral research in immunotherapy for Multiple Myeloma. During his research term he presented his research work at numerous conferences. In December 2012 he won the best scientific poster award at the Internal Medicine Day, MUMC+. Since November 2013, he is pursuing further post-doctoral research in the field of Multiple Myeloma at DanaFarber Cancer Institute, affiliate Harvard Medical School in Boston.

\section{Publication}

Hypoxia induced impairment of NK cell cytotoxicity against Multiple Myeloma can be overcome by IL-2 activation of the NK cells. Sarkar S, Germeraad WT, Rouschop KM, Steeghs EM, van Gelder M, Bos GM, Wieten L. PLoS One. 2013;8:e64835. 
Valorization 


\section{Valorization}

In 2002, extremely promising data showed that Natural Killer cells can be a great therapeutic potential for treatment of Acute Myeloid Leukemia (AML). Subsequently different haematological and solid tumor's are currently under study for determining the clinical potential of NK cells to cure the disease of Cancer.

The studies conducted in this doctoral thesis pave way for a better understanding for the selection and optimization of Natural Killer cell therapy protocol for Multiple Myeloma. Key tumor microenvironmental factors, such as hypoxia can completely nullify the effects of NK cells, and in this thesis we describe ways of eliminating such intrinsic immune-evasion strategies of the myeloma microenvironment. The novel strategies proposed in the thesis, in the context of optimal selection of Natural Killer cells, based on the MHC class I expression and HLA-E expression of the donor myeloma cells, will be a cornerstone in the planned allogeneic Natural Killer cell therapy for Multiple Myeloma. Successful treatment of Myeloma patients will be expensive, as it is a form of cellular therapy, costing 25,000-50,000 euros per regime. However on the longer run, it is expected to decrease in costs. In particular, the data shown in Chapter 4, suggest that a combination of chemotherapy-radiotherapy with Natural Killer cells offers a curative regimen for Myeloma, and this approach already enables us to significantly reduce the treatment costs, when compared to other medical center's developing NK cell therapy. Though expensive, saving a human life, and curing them from cancer, can never be weighed against the value of economic burden.

Furthermore, since Myeloma is a disease of the elderly, the NK cell therapy developed in clinical practice will be mostly targeting the older people in the age group of $>65$ years. Saving the lives of these people, will have a huge impact of the gross domestic happiness of a country such as Netherlands.

Future clinical trial is needed to test the efficacy of the NK cell products that will be developed at MUMC+. However with the data shown in this thesis, we are extremely hopeful about the clinical success of the NK cells for Myeloma before the end 2020. By 2030, after 10 year follow up, we expect allogeneic NK cell therapy to be a standard curative regimen for patients with Myeloma. 
